\title{
ADVANCING THE METHODOLOGY FOR PREDICTING THE SAFETY EFFECTS OF HIGHWAY DESIGN AND OPERATIONAL ELEMENTS
}

By

\author{
Taha Saleem \\ Master of Applied Science, Ryerson University, 2012 \\ Bachelor of Applied Science, University of Toronto, 2010 \\ A dissertation \\ presented to Ryerson University \\ in partial fulfillment of the \\ requirements of the degree of \\ Doctor of Philosophy \\ in the Program of \\ Civil Engineering
}

Toronto, Ontario

(C) Taha Saleem 2016 


\section{Author's Declaration}

I hereby declare that I am the sole author of this dissertation. This is a true copy of the dissertation, including any required final revisions, as accepted by my examiners.

I authorize Ryerson University to lend this dissertation to other institutions or individuals for the purpose of scholarly research

I further authorize Ryerson University to reproduce this thesis by photocopying or by other means, in total or in part, at the request of other institutions or individuals for the purpose of scholarly research.

I understand that my dissertation may be made electronically available to the public.

Taha Saleem

Department of Civil Engineering

Ryerson University 


\title{
“ADVANCING THE METHODOLOGY FOR PREDICTING THE SAFETY EFFECTS OF HIGHWAY DESIGN AND OPERATIONAL ELEMENTS”
}

\author{
By \\ Taha Saleem \\ Doctor of Philosophy \\ Civil Engineering \\ Ryerson University
}

2016

\begin{abstract}
$\underline{\text { Abstract }}$
Road traffic crashes are one of the major causes of deaths and injuries around the world killing approximately 1.2 million people and injuring over 50 million every year. One of the primary goals of transportation agencies around the world is to reduce crashes as well as minimize the potential for human error and provide a forgiving road environment. Estimating the safety effects of highway design and operational elements is essential in achieving this goal. This research is divided into two components aimed at advancing the methodology for estimating these effects. The first component looks at evaluating the potential of cross-sectional analysis for developing crash modification factors/functions (CMFs/CMFunctions) used to represent the effects of safety treatments on crashes. First, the cross-sectional approach was used to investigate the safety effects of horizontal curvature on rural two-lane highways, which would be impossible to evaluate with before-after data. Second, this approach was further evaluated using databases of sites that were actually treated and similar, but untreated reference sites. The treatment databases for this part consisted of combination rumble strips (center line plus
\end{abstract}


shoulder), wet reflective pavement markings and intersection conflict warning systems. The results from the cross-sectional analysis were then compared to recent studies where empirical Bayes before-after analysis was conducted with the same dataset used for the cross-sectional analysis. The results were promising in that the effects from the cross-sectional and beforeafter studies were reasonably comparable in each case. In addition, it was possible in some cases to relate the CMF to application circumstances by developing CMFunctions, providing results that could not be achieved in the before-after studies. The second component of this research involved development of roundabout crash predictions using conflicting volumes and delays, which could only be estimated from turning movement counts that are rarely collected at roundabouts. The object was to determine whether the considerable extra effort to collect these data would be worthwhile. The developed models were compared to the traditional models based on approach flows. The results suggest that collecting turning movement data, which is also required for capacity analysis, would be worthwhile for evaluating roundabout safety. 


\section{Acknowledgements}

I would first of all like to thank my family for all of their support during this time; without their support this dissertation could not have been possible.

I would also like to thank my supervisor Dr. Bhagwant Persaud for all the educational and financial support provided during this time.

I would also like to thank Bob Henderson from the Region of Waterloo, the administrative staff at the Highway Safety Information Systems, and the various US Departments of Transportation for providing the data without which this research would not have been possible.

Lastly, I would like to thank all of my colleagues and friends for supporting me and encouraging me during all the tough times. 


\section{$\underline{\text { Table of Contents }}$}

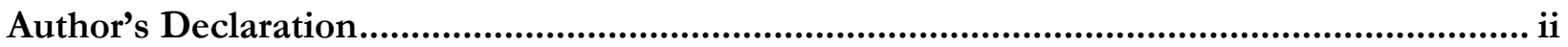

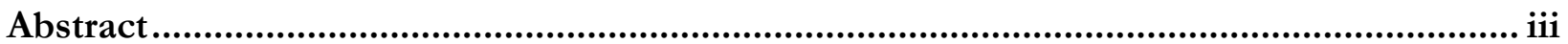

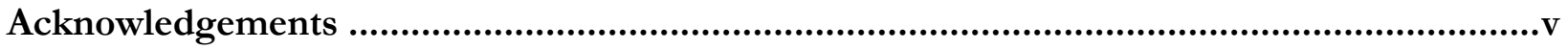

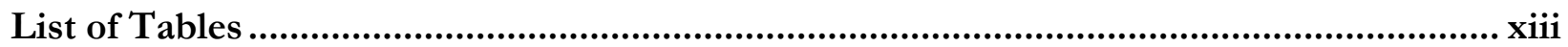

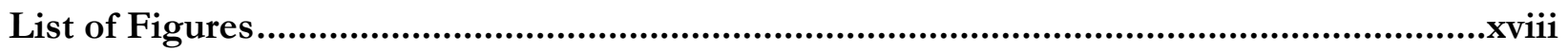

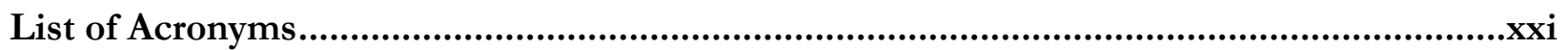

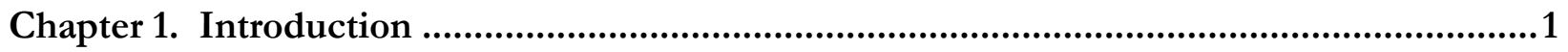

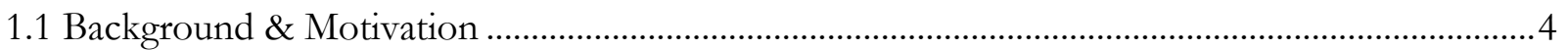

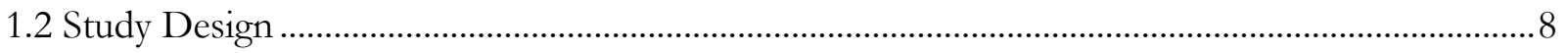

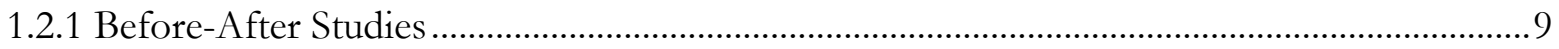

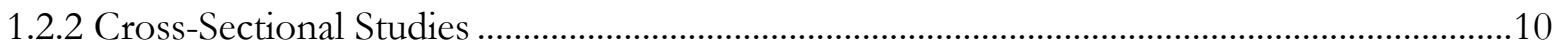

1.3 The Role of Crash Modification Factors or Functions ..............................................................11

1.4 Current State of Crash Modification Factor/Function Research ...............................................13

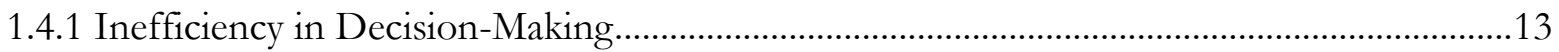

1.4.2 Differences Between What Can be Observed and What Needs to be Known About Crash

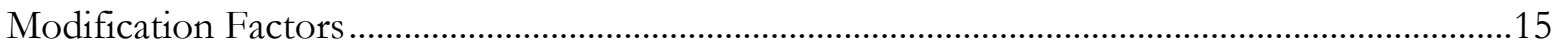

1.4.3 Transferability of Crash Modification Factors/Functions .....................................................15

1.4.4 Crash Modification Factor as a Random Variable ...................................................................16 
1.5 Issues with Current Research

1.5.1 Need for CMFunctions

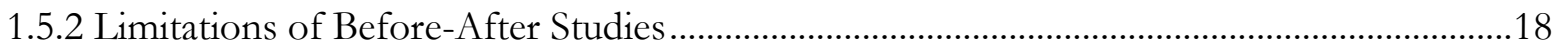

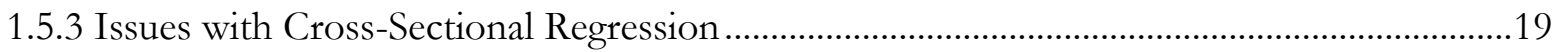

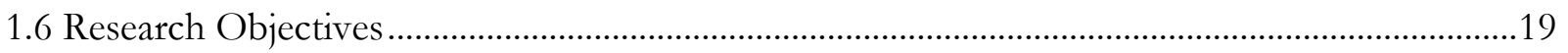

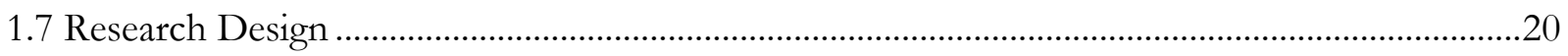

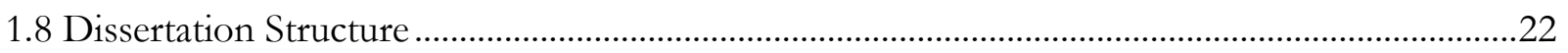

Chapter 2. Review of Current Literature on Crash Modelling .............................................24

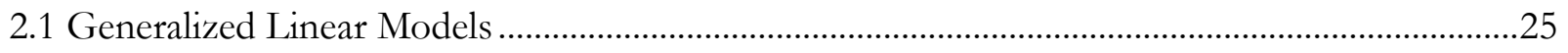

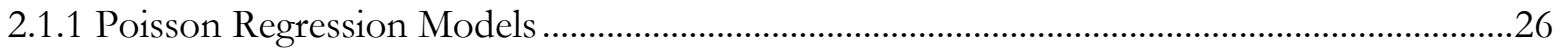

2.1.2 Negative Binomial (Poisson Gamma) Regression Models ....................................................28

2.1.3 Poisson Lognormal Regression Models .................................................................................29

2.1.4 Zero Inflated Regression Models ......................................................................................... 30

2.2 Generalized Estimating Equations ………...................................................................................

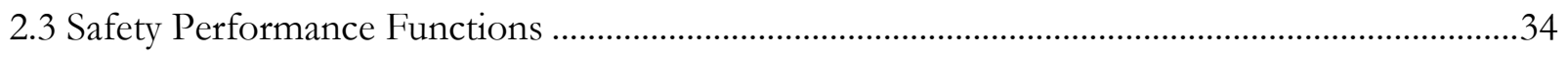

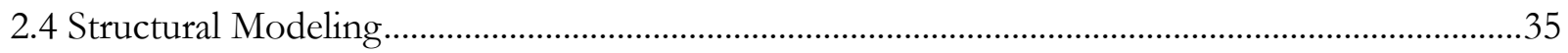

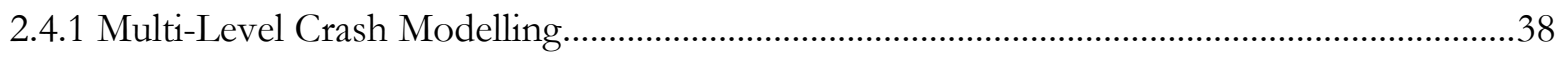

2.5 Cross-Sectional Regression to Estimate CMFs/CMFunctions ......................................................40

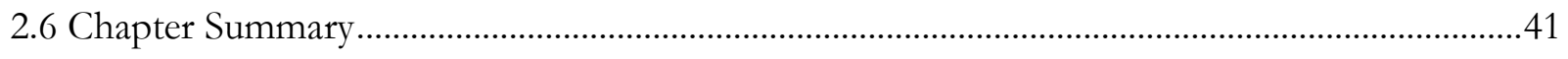




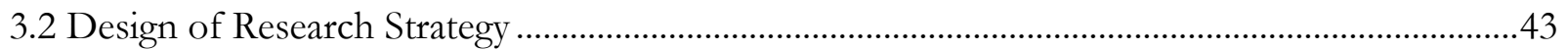

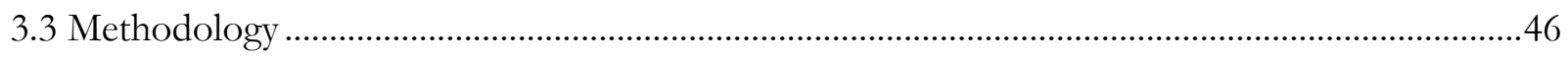

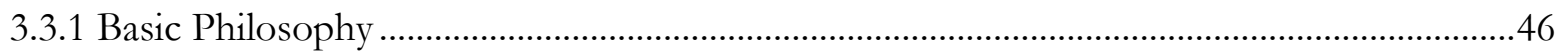

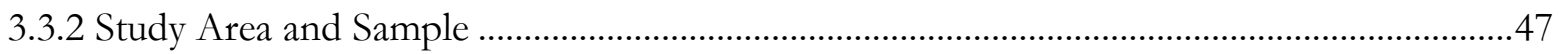

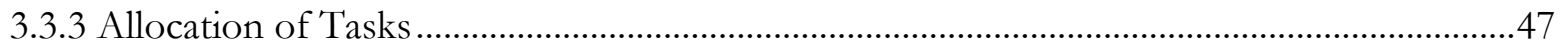

3.3.3.1 Tracing and Collecting the Latest Developments of CMFs and CMFunctions............47

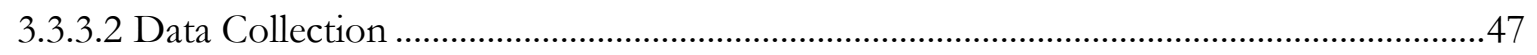

3.3.3.3 Constructing a General SPF and CMFunction Configuration .......................................48

3.3.3.4 Re-Calibrating Current Models to Convert CMFs to CMFunctions ...............................48

3.3.3.5 Using Cross-Sectional Data and Before-After Data to Generate CMFs and

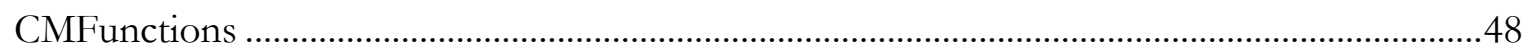

3.3.3.6 Developing New Approaches to Estimate Safety of an Entity .....................................48

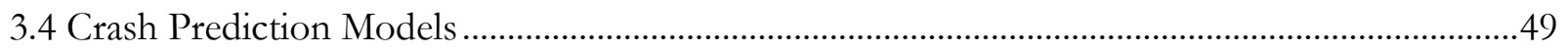

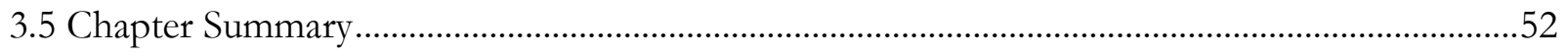

Chapter 4. Evaluating the Potential of Cross-Sectional Analysis for Developing CMFs in the Absence of Before-After Data - Case Study on the Safety Effects of Horizontal Curvature..53

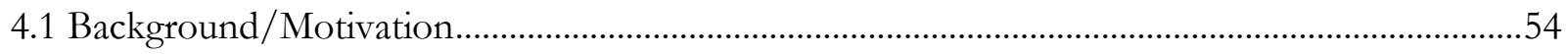

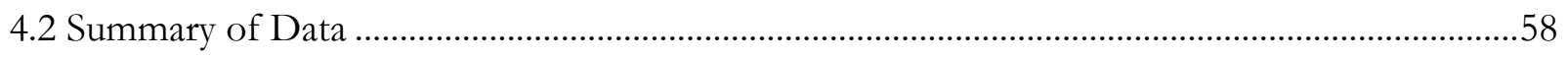


4.3 Model Fitting and Evaluation

4.3.1 Model Fitting Using Curve Data

4.3.2 Model Fitting Using Up and Downstream Tangent Data

4.4 Application of Crash Prediction Models for Estimating Crash Modification Factors for Curve Flattening .75

4.4.1 Crash Reduction Rate (CRR) \& Crash Modification Factor (CMF) Estimation Methodology .76

4.4.2 CMF Estimates for Incrementally Increasing Radius over the Minimum Value Required at Certain Design Speeds .77

4.5 Chapter Summary. .85

\section{Chapter 5. Evaluating the Potential of Cross-Sectional Analysis to Develop CMFs and} CMFunctions using Before-After Study Databases ...........................................................8 87

5.1 Safety Evaluation of Combination Rumble Strips

5.1.1 Background/Motivation

5.1.2 Summary of Data

5.1.3 Model Fitting and Evaluation .93

5.1.3.1 Model Fitting Using Kentucky Data.....

5.1.3.1.1 Modelling the Presence of Combination Rumble Strips - Model A...... .94

5.1.3.1.2 Modelling the Interaction of Combination Rumble Strips with Shoulder Width-Model B........97

5.1.3.2 Model Fitting Using Pennsylvania Data..... 101

5.1.3.2.1 Modelling the Presence of Combination Rumble Strips - Model A. 
5.1.3.2.2 Modelling the Interaction of Combination Rumble Strips with Shoulder Width - Model B..... 104

5.1.3.3 Model Fitting Using Combined (Kentucky + Pennsylvania) Data .............................. 108

5.1.3.3.1 Modelling the Presence of Combination Rumble Strips - Model A......................................... 108

5.1.3.3.2 Modelling the Interaction of Combination Rumble Strips with Shoulder Width - Model B..... 111

5.1.4 Application of Crash Prediction Models for Estimating Crash Modification Factors for

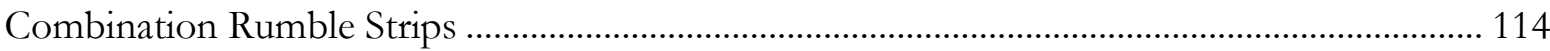

5.1.5 Summary of the Combination Rumble Strip Case Study ................................................. 120

5.2 Safety Evaluation of Wet Reflective Pavement Markings ......................................................... 121

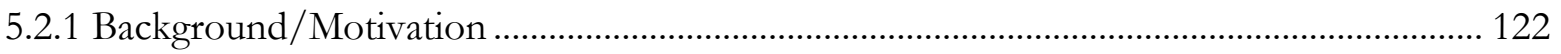

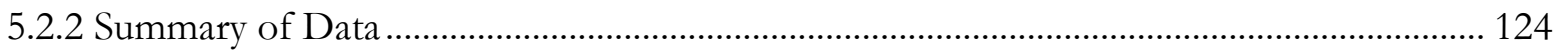

5.2.3 Model Fitting and Evaluation .............................................................................................. 127

5.2.3.1 Model Fitting Using North Carolina Data ..................................................................... 127

5.2.3.1.1 Modeling the Presence of Wet Reflective Pavement Markings - Model A .............................. 127

5.2.3.1.2 Modelling the Interaction of Wet Reflective Pavement Markings with Number of Lanes - Model

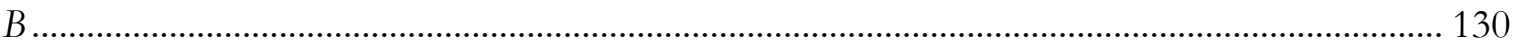

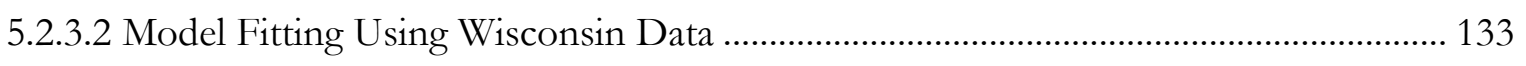

5.2.4 Application of Crash Prediction Models for Estimating Crash Modification Factors for

Wet Reflective Pavement Markings .............................................................................................. 136

5.2.5 Summary of the Wet-Reflective Pavement Marking Case Study ....................................... 141

5.3 Safety Evaluation of Intersection Conflict Warning Systems ................................................. 142 
5.3.4 Application of Crash Prediction Models for Estimating Crash Modification Factors for

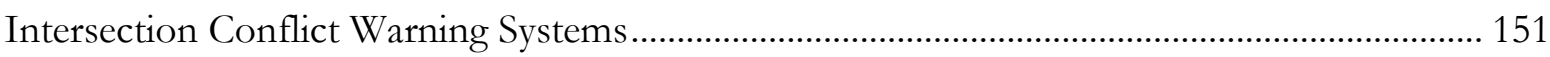

5.3.5 Summary of the Intersection Conflict Warning Case Study............................................... 154

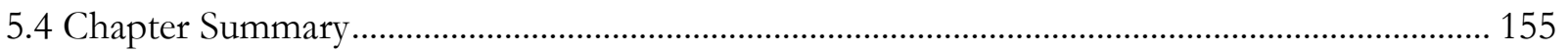

\section{Chapter 6. Investigation of New Approaches to Estimating Safety Performance of}

Roundabouts ............................................................................................................................. 158

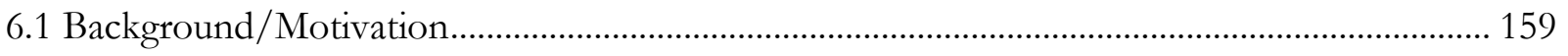

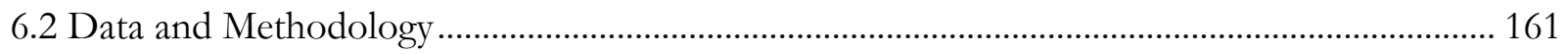

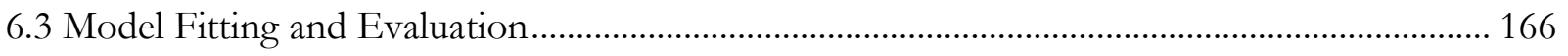

6.3.1 Multi-Lane Roundabout Models ....................................................................................... 166

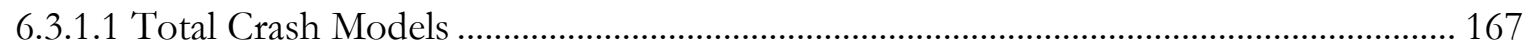

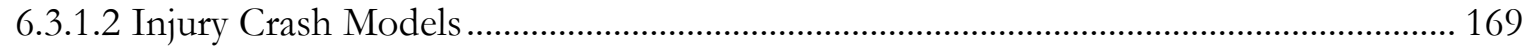

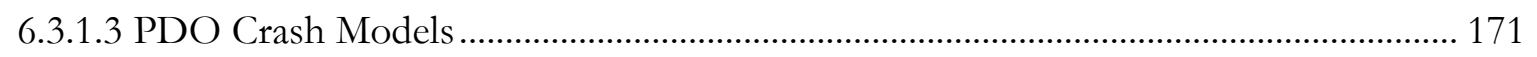

6.3.2 Single-Lane Roundabout Models ..................................................................................... 174

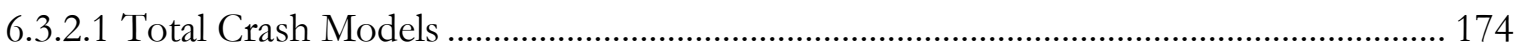




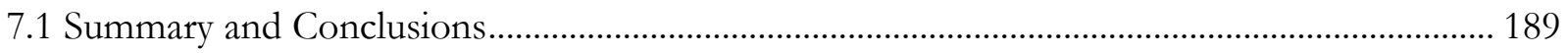

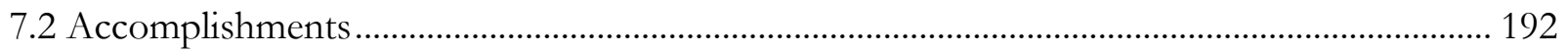

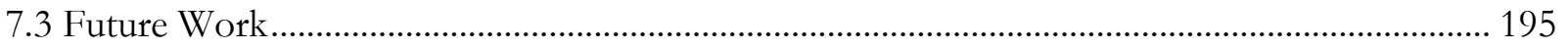

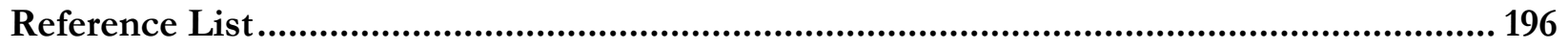




\section{$\underline{\text { List of Tables }}$}

Table 4.1 Highway Safety Manual CMFs for Grade of Roadway Segments. .56

Table 4.2 Summary Statistics for the Curved Segments.

Table 4.3 Summary Statistics for the Tangent Sections .60

Table 4.4 Crash Statistics for the Curved Segments

Table 4.5 Crash Statistics for the Tangent Sections .61

Table 4.6 Estimates \& Goodness of Fit Measures for the Curve Models on Level Grades

Table 4.7 Estimates \& Goodness of Fit Measures for the Curve Models on Moderate Grades

Table 4.8 Estimates \& Goodness of Fit Measures for the Curve Models on Steep Grades

Table 4.9 Estimates \& Goodness of Fit Measures for the Tangents on Level Grades.....

Table 4.10 Estimates \& Goodness of Fit Measures for the Tangents on Moderate Grades

Table 4.11 Estimates \& Goodness of Fit Measures for the Tangents on Steep Grades.... .70

Table 4.12 Total Crashes CMF Estimations for Certain Increases in the Radius beyond Minimum

Values (Segments on Level Grades).

Table 4.13 Total Crashes CMF Estimations for Certain Increases in the Radius beyond Minimum

Values (Segments on Moderate Grades) 
Table 4.14 Total Crashes CMF Estimations for Certain Increases in the Radius beyond Minimum

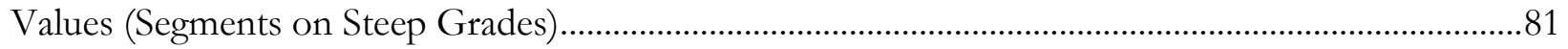

Table 4.15 Average Crash Reduction Rate (CRR) from the Results in Table 4-12 - 4-14...................83

Table 5.1 Summary Statistics for Kentucky Sites …........................................................................... 92

Table 5.2 Crash Statistics for Kentucky Sites ....................................................................................... 92

Table 5.3 Summary Statistics for Pennsylvania Sites ...........................................................................93

Table 5.4 Crash Statistics for Pennsylvania Sites .................................................................................. 93

Table 5.5 Estimates and Dispersion Parameters for Kentucky Model A.............................................95

Table 5.6 Goodness of Prediction Measures for Kentucky Model A....................................................95

Table 5.7 Estimates and Dispersion Parameters for Kentucky Model B .............................................98

Table 5.8 Goodness of Prediction Measures for Kentucky Model B …................................................99

Table 5.9 Estimates and Dispersion Parameters for Pennsylvania Model A..................................... 102

Table 5.10 Goodness of Prediction Measures for Pennsylvania Model A......................................... 102

Table 5.11 Estimates and Dispersion Parameters for Pennsylvania Model B ................................... 106

Table 5.12 Goodness of Prediction Measures for Pennsylvania Model B ........................................ 106

Table 5.13 Estimates and Dispersion Parameters for Combined Model A ...................................... 109 
Table 5.14 Goodness of Prediction Measures for Combined Model A 110

Table 5.15 Estimates and Dispersion Parameters for Combined Model B 112

Table 5.16 Goodness of Prediction Measures for Combined Model B 113

Table 5.17 Comparison of CMF Estimates with CMFs from Persaud et al. (2016). 115

Table 5.18 Summary Statistics for North Carolina Freeway Data 125

Table 5.19 Crash Statistics for North Carolina Freeway Data..... 125

Table 5.20 Summary Statistics for Wisconsin Multi Lane Data. 126

Table 5.21 Crash Statistics for Wisconsin Multi Lane Data 126

Table 5.22 Estimates and Dispersion Parameters for North Carolina Model A 128

Table 5.23 Goodness of Prediction Measures for North Carolina Model A. 128

Table 5.24 Estimates and Dispersion Parameters for North Carolina Model B 131

Table 5.25 Goodness of Prediction Measures for North Carolina Model B 131

Table 5.26 Estimates and Dispersion Parameters for Wisconsin Models. 134

Table 5.27 Goodness of Prediction Measures for Wisconsin Models 135

Table 5.28 Comparison of Freeway CMF Estimates with CMFs from Lyon et al. (2016)........ 137 
Table 5.29 Comparison of Multi-Lane Divided CMF Estimates with CMFs from Lyon et al. (2016)

Table 5.30 Comparison of Wet Reflective Pavement Marking CMFs for Varying Number of Lanes

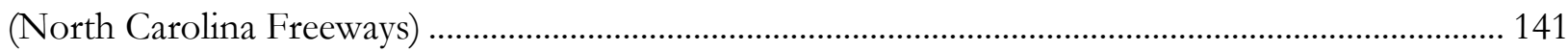

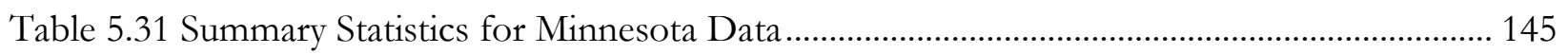

Table 5.32 Summary Statistics for Missouri Data .............................................................................. 145

Table 5.33 Summary Statistics for North Carolina Data ...................................................................... 146

Table 5.34 Estimates and Dispersion Parameters for Two-Lane Model........................................... 147

Table 5.35 Goodness of Prediction Measures for Two-Lane Model.................................................. 148

Table 5.36 Estimates and Dispersion Parameters for Four-Lane Model .......................................... 149

Table 5.37 Goodness of Prediction Measures for Four-Lane Model ................................................... 150

Table 5.38 Comparison of 2-Lane CMF Estimates with CMFs from Himes et al. (2016)............... 152

Table 5.39 Comparison of 4-Lane CMF Estimates with CMFs from Himes et al. (2016)............... 153

Table 6.1 Summary Statistics for Region of Waterloo Multi-Lane Roundabouts ............................. 164

Table 6.2 Summary Statistics for City of Ottawa Single-Lane Roundabouts.................................... 165

Table 6.3 Summary Statistics for Washington State Multi-Lane Roundabouts .................................. 165

Table 6.4 Summary Statistics for Region of Waterloo Single-Lane Roundabouts ............................ 165 
Table 6.5 Total Crashes (By Years) Recorded at Sites Used for Analysis 166

Table 6.6 Estimates and Goodness of Fit Measures for Total Crash Models 167

Table 6.7 Estimates and Goodness of Fit Measures for Injury Crash Models. 170

Table 6.8 Estimates and Goodness of Fit Measures for PDO Crash Models...

Table 6.9 Estimates and Goodness of Fit Measures for Total Crash Models.. 175

Table 6.10 Estimates and Goodness of Fit Measures for Injury Crash Models.

Table 6.11 Estimates and Goodness of Fit Measures for PDO Crash Models. 179

Table 6.12. Estimates for Multi-Lane GEE Model (with/without Trend)... 183

Table 6.13 Estimates for Single-Lane GEE Model (with/without Trend)..... 185 


\section{$\underline{\text { List of Figures }}$}

Figure 1.1 Reference and Treated Group..............................................................................................

Figure 1.2 Logical Framework for Before-after Evaluations ..............................................................10

Figure 3.1 Classification of Research Sub-Objectives ............................................................................4

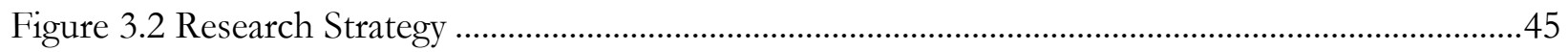

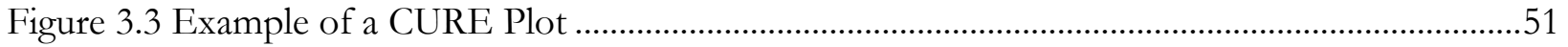

Figure 4.1 CURE Plots for Total Crashes (Curved Segments) ...........................................................64

Figure 4.2 CURE Plots for F\&I Crashes (Curved Segments) ................................................................65

Figure 4.3 CURE Plots for PDO Crashes (Curved Segments) ..............................................................66

Figure 4.4 CURE Plots for Total Crashes (Tangent Sections)................................................................

Figure 4.5 CURE Plots for F\&I Crashes (Tangent Sections) .............................................................72

Figure 4.6 CURE Plots for PDO Crashes (Tangent Sections) .............................................................73

Figure 4.7 Illustration of Curve Flattening (going from 1-2-2'-1' to 1-I-1') for a given Deflection

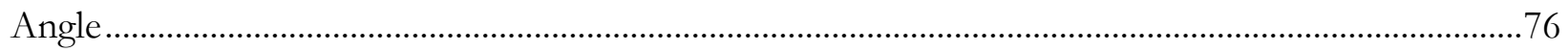

Figure 4.8 Comparison of CMFs for Varying Radii on Level Grade $(2 \%)$............................................84

Figure 4.9 Comparison of CMFs for Varying Radii on Moderate Grade (5\%) ...................................84 
Figure 4.10 Comparison of CMFs for Varying Radii on Steep Grade (8\%).......

Figure 5.1 Cure Plots for Kentucky Model A.

Figure 5.2 Cure Plots for Kentucky Model B

Figure 5.3 CURE Plots for Pennsylvania Model A 104

Figure 5.4 CURE Plots for Pennsylvania Model B. 107

Figure 5.5 CURE Plots for Combined Model A 110

Figure 5.6 CURE Plots for Combined Model B 113

Figure 5.7 Bar Graphs for the Comparison of Rumble Strips CMFs. 117

Figure 5.8 Comparison of CMFs for Varying Shoulder Width (Kentucky)...... 119

Figure 5.9 Comparison of CMFs for Varying Shoulder Width (Pennsylvania)..... 119

Figure 5.10 Comparison of CMFs for Varying Shoulder Width (Combined Data) 120

Figure 5.11 CURE Plots for North Carolina Model A

Figure 5.12 CURE Plots for North Carolina Model B

Figure 5.13 CURE Plots for Wisconsin Models 135

Figure 5.14 Bar Graph for the Comparison of Freeway CMFs. 139

Figure 5.15 Bar Graph for the Comparison of Multi-Lane Divided CMFs 140 
Figure 5.16 CURE Plots for Two-Lane Model 148

Figure 5.17 CURE Plots for Four-Lane Model... 150

Figure 5.18 Bar Graphs for the Comparison of Two- \& Four-Lane CMFs....... 154

Figure 6.1 CURE Plots for all Model Types (Total Crashes). 168

Figure 6.2 CURE Plots for all Model Types (Injury Crashes) 170

Figure 6.3 CURE Plots for all Model Types (PDO Crashes). 173

Figure 6.4 CURE Plots for all Model Types (Total Crashes)..... 176

Figure 6.5 CURE Plots for all Model Types (Injury Crashes). 178

Figure 6.6 CURE Plots for all Model Types (PDO Crashes). 179 


\section{List of Acronyms}

\begin{tabular}{|c|c|}
\hline$A A D T$ & Annual Average Daily Traffic \\
\hline$C M F$ & Crash Modification Factors \\
\hline CMFunction & Crash Modification Functions \\
\hline CURE & Cumulative Residuals \\
\hline$F \& I$ & Fatal and Injury Crashes \\
\hline GEE & General Estimating Equations \\
\hline GLM & Generalized Linear Modeling \\
\hline GOF & Goodness-Of-Fit \\
\hline HEO & Head On Crashes \\
\hline ICWS & Intersection Conflict Warning Systems \\
\hline$K$ & Over Dispersion Parameter \\
\hline$M A D$ & Mean Absolute Deviation \\
\hline$M S P E$ & Mean Squared Prediction Error \\
\hline$M P E$ & Mean Prediction Error \\
\hline$N B$ & Negative Binomial \\
\hline$P D O$ & Property Damage Only Crashes \\
\hline $\operatorname{Pr}$ & Significance Level (p-value) \\
\hline
\end{tabular}


Run off Road Crashes

SAS

Statistical Analysis Software

$S P F$

Safety Performance Function

$S S S D$

Sideswipe Same Direction Crashes

$S S O D$

Sideswipe Opposite Direction Crashes

$W R P M$

Wet Reflective Pavement Markings 


\section{Chapter 1. Introduction}

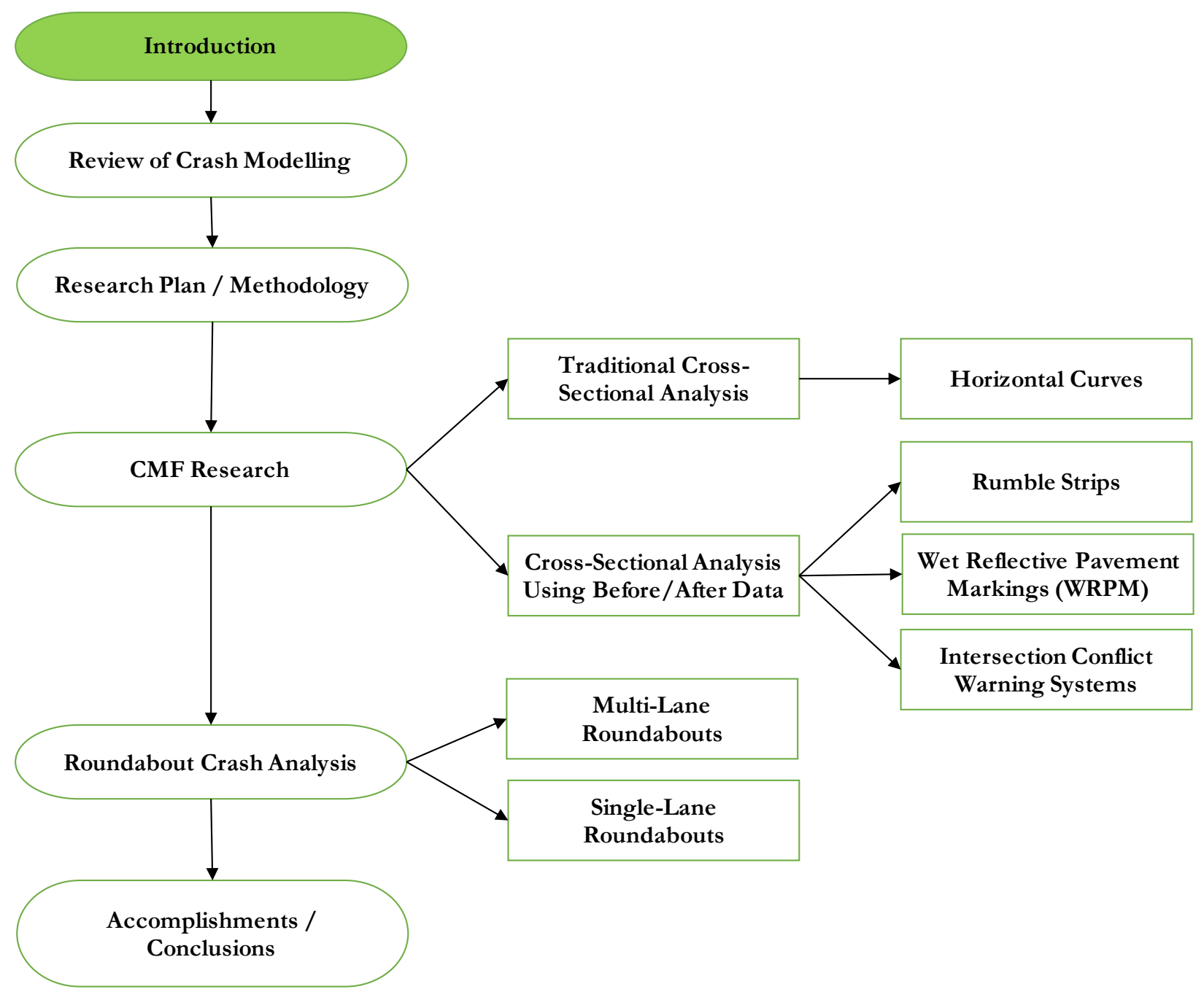

Road traffic crashes are one of the major causes of deaths and injuries around the world. Approximately 1.2 million people (2.1\% of all deaths) are killed every year and over 50 million are injured or disabled worldwide due to road traffic crashes (World Health Organization, 2009). Furthermore, the World Health Organization's (WHO) data shows that road traffic crashes are the second leading cause of injury, death, or disability after HIV/AIDS worldwide for the people in the age group of 15-44 years (World Health Organization, 2009). The WHO 
Global Burden of Disease model also predicts that by 2020, road traffic deaths could rise to 2.34 million/year worldwide, with under developed countries seeing an increase of approximately $80 \%$ and developed countries seeing a reduction of about 30\% (World Health Organization, 2009). The decrease in road traffic deaths in developed countries can be attributed to many factors such as increased awareness amongst people and strict government policies.

According to Transport Canada's Road Safety Vision, Canadian roads are amongst the top 10 safest roads in the world (Transport Canada, 2006). Despite having some of the safest roads in the world, everyday in Canada there are approximately 8 deaths, 600 injuries, 1,600 crashes costing a whopping \$27 million to the society (Transport Canada, 2006). Between 1984 and 2006, Canadian roads saw a decrease of $33 \%$ in deaths resulting from traffic crashes and a decrease of $35 \%$ in serious injuries resulting from traffic crashes (Transport Canada, 2006). Even after all the decreases, in 2012, Transport Canada (Transport Canada, 2013) reported that 2700 people were killed and 10,315 were seriously injured as a result of traffic crashes. The implementation of the Road Safety Vision aims at reducing the number of deaths and serious injuries by a further $30 \%$ through implementation of various recommendations (Transport Canada, 2006). Roads in Ontario are amongst the safest in both Canada and North America (Patterson, 2009). Statistics show that the fatality rates in car crashes in Ontario have dropped to 0.87 per 10,000 licensed drivers (Patterson, 2009). Even though the roads in Ontario are amongst the safest in Canada, the collisions still generate high numbers in social costs.

On average, road traffic crashes cost countries between $1 \%$ and $3 \%$ of their gross domestic product (GDP) (World Health Organization, 2009). According to Transport Canada's report 
Analysis and Estimation of the Social Cost of Motor Vehicle Collisions in Ontario, motor vehicle crashes in Ontario in 2004 generated about $30 \%$ of social costs of all Canadian crashes or about 3.5\% of Ontario's 2004 GDP (Transport Canada, 2007). In addition to placing a burden on the national GDP, these crashes also cause great emotional and financial stress to the millions of families that are affected by them. A lot of these crashes can be prevented by implementing effective road safety measures. To be able to select the best measure, a decision maker needs information about the effectiveness of different measures. Moreover, information about the effectiveness of measures is needed in case one (e.g., a politician) has to make a choice between expenses on road safety measures and other expenses, like measures to limit environmental effects of traffic or measures to improve traffic throughput (OECD, 2012).

Generally, countries try to establish a clear understanding of the costs and benefits of preventing road crashes. Decision makers need to choose between a variety of safety measures and understanding the effectiveness of different safety treatments and strategies (often referred to as Crash Modification Factors or Functions) will help in making better decisions (OECD, 2012). Hence, Crash Prediction Models are one of the tools to help professionals make safety decisions. Crash prediction models consist of Safety Performance Functions (SPFs) and Crash Modification Factors or Functions (CMFs). SPFs are "an equation used to estimate or predict the expected average crash frequencies per year at a location as a function of traffic volume and in some cases roadway or intersection characteristics" (AASTHO, 2010). SPFs are needed to apply CMFs to see how an entity is performing. They are used for various different applications such as assessing whether safety performance of an entity is as expected, network screening, and determining the effect of treatment. Hence, SPFs are required to apply the 
CMFs to see if an entity is performing as expected or to determine the effect of a treatment or a decision in the design process. This research addresses advancing both SPFs and CMFs with a focus on CMFs.

\subsection{Background \& Motivation}

There are two main types of analysis conducted in the domain of safety evaluation research, namely, before-after and cross-sectional. Safety effects from before-after analysis are based on the comparison of safety performance before and after the implementation of a treatment, whereas, safety effects from cross-sectional analysis are based on the comparison in the safety performance of sites that have a specific feature with those that do not (Wu et al., 2014). These methods will be discussed in further detail in Section 1.2.

The main purpose of these analyses is to derive crash modification factors/functions (CMFs/CMFunctions) in order to be able to represent the safety effects of applied/proposed treatments. According to Carter et al. (2012), "There are essentially two ways of representing the effects of safety treatments on crashes. One is through the use of crash modification factors (CMFs) and the other is through the use of crash modification functions (CMFunctions)."

CMFs are usually represented using point estimates. For example, a CMF of 0.68 for a particular treatment " $\mathrm{A}$ " would indicate on average a 32\% reduction in crashes. Conversely, a CMF of 1.12 for a particular treatment "B" would indicate on average a $12 \%$ increase in crashes. Based on a CMF and its crash reduction rate (CRR), which is usually calculated as, $1-C M F$, one can get a first-hand look at whether the treatment of interest is effective in improving safety or not. For instance, in the examples noted above treatment "A" has a CRR 
of 0.32 and treatment "B" has a CRR of -0.12 indicating a negative impact of treatment " $\mathrm{B}$ " on crashes.

In many cases, the effect of a treatment may depend on several factors, including the traffic volume and other road characteristics. In such cases, the use of CMFunctions is more appropriate than using CMFs. An example of a CMFunction can be as follows:

CMFunction for treatment " $X$ " $=e^{0.0152(L / W)}$

In this case, the variables $\mathrm{L}$ and $\mathrm{W}$ would represent a road characteristic such as the number of lanes and the shoulder width.

The use of CMFunctions helps to clearly identify the effects of treatment on all the different sections of a roadway and will also help in minimizing the prediction bias (Elvik, 2011a). For example, a CMF of 0.8 for adding a lane to a two lane road segment would indicate an average reduction of crashes once the third lane is added, but will not capture the effect of varying shoulder widths, presence of horizontal curves, etc. A CMFunction would ensure that one is able to measure the safety improvements accurately at all the different sections of the roadway.

Deriving a CMFunction may be much easier established using cross-sectional analysis considering its ability to model interactions between the treatment of interest and various other road characteristics. On the other hand, similar effects could also be established using beforeafter analysis, but this would require a large number of before-after applications over various different circumstances. Due to the nature of the data available for road safety research, it is very hard or nearly impossible to get enough of these data in order to properly relate the CMF to application circumstances. Various researchers such as Gross et al. (2011), Lord et al. 
(2012), and $\mathrm{Wu}$ et al. (2014) have argued that before-after studies do not provide a straightforward answer to deriving CMFunctions.

On the other hand, before-after studies can accurately provide the single value CMF for a treatment effect as it would have materialized in real-life. Hauer (2014) strongly argues that regression analysis based on cross-sectional data cannot be trusted to predict the effect of the cause. However, in an unpublished paper, he suggests that an approach by Bonneson et al. (2008) may be viable. In this approach, compared road segments share the same traffic, environment, and road users but differ in the Property of Interest, as quoted by Bonneson et al. (2008), "the procedure is based on the use of matched pairs of road segments. The segment pairs ... are selected such that their attributes are identical, except for differences in the attributes (of interest) ... By selecting pairs of matched segments, the effect of the selected attributes on safety is isolated and all other factors are controlled". Current research lags behind in evaluating the potential of cross-sectional analysis for predicting CMFs/CMFunctions, and also there has been little previous research that looks into investigating whether $\mathrm{CMFs} / \mathrm{CMFunctions}$ derived from cross-sectional regression models reflect the true safety effects of corresponding treatments (Wu et al., 2014).

As previously mentioned in this section, $\mathrm{CMFs} / \mathrm{CMFunctions}$ are one side of the puzzle. Crash prediction models consist of both SPFs and CMFs. CMFs are based on the change in crashes due to the treatment, SPFs on the other hand provide a way to link crashes to traffic characteristics. These could be as simple as using the traffic volumes or, for intersections, one can go a step further and use number of conflicts, conflicting volumes and delays; other viable characteristics as are defined in the Highway Capacity Manual (TRB, 2010). In order to derive 
these characteristics, one would potentially need to make use of turning movement counts rather than just the entering volumes on the major and minor approaches.

Turning movement counts can be easily collected at any signalized or un-signalized intersection, but are rarely ever collected at roundabouts. According to the National Cooperative Highway Research Program (NCHRP) Report 672: "Roundabouts: An Informational Guide" (TRB, 2010a), applications of roundabouts have been gaining popularity in the recent past mostly due to the fact that roundabouts have shown to reduce injury crashes by about $76 \%$ compared to signalized and stop controlled intersections. Given the geometry of roundabouts, collecting the turning movement counts would require considerable extra effort than counting approach volumes. The current research on roundabouts has been focusing heavily on improving the design of the roundabouts to make vehicle navigation through it smoother and the issue of using turning movement counts to better predict safety at roundabouts has not gained momentum even though these counts are required for capacity analysis using the Highway Capacity Manual (TRB, 2010) methodology.

The main purpose of this research is to improve the prediction methodology for estimating the safety impacts of highway design and operational elements by focusing on; (1) the potential of cross-sectional analysis to better estimate the safety effects of proposed/implemented countermeasures for cases where before-after data is not available and to compare the results and better estimate CMFunctions where before-after data is available, and (2) whether extra effort put into collecting traffic data can be used to better predict safety, in particular at roundabouts. 


\subsection{Study Design}

Study design falls into two categories: experimental and observational. Experimental studies are planned studies in which sites are selected at random for treatment. According to Elvik (2011b), experimental studies are the most rigorous way to establish causality. Experimental studies evaluate safety improvements of implemented treatments solely for the purpose of measuring their effectiveness and are not very common somewhat because of the potential liabilities involved. On the other hand, observational studies are not planned and are more common in research because they consider safety improvements to improve the roadway system (Carter et al, 2012).

Observational studies can be broadly classified into before-after and cross-sectional studies. Before-after studies include "all techniques by which one may study the safety effect of some change that has been implemented on a group of entities (road sections, intersections, drivers, vehicles, neighborhoods, etc.)" (Hauer, 1997). On the other hand, cross-sectional studies include those where "one is comparing the safety of one group of entities having some common feature (say, STOP controlled intersections) to the safety of a different group of entities not having that feature (say, YIELD controlled intersections), in order to assess the safety effect of that feature (STOP versus YIELD signs)" (Hauer, 1997).

Before-after studies are preferred because CMFs or CMFunctions derived from these are based on the change in safety due to the implementation of a treatment. On the other hand, crosssectional studies, though not preferred, are necessary because CMFunctions are difficult to derive from limited before-after data (before-after data is also sometimes impossible to 
acquire) and can potentially be derived using cross-sectional data considering interactions between the element of interest and other road features/characteristics.

\subsubsection{Before-After Studies}

CMFs or CMFunctions derived from before-after studies are based on the change in safety due to the implementation of a treatment. The most practically established approaches for beforeafter evaluations are the Empirical Bayesian method (EB) and the Full Bayesian method (FB). Both the EB and FB approaches associate a reference group which is similar to treated sites (treated group) and is introduced to offer referential information for before-after evaluations, as illustrated in Figure 1.1 (Chen, 2013).

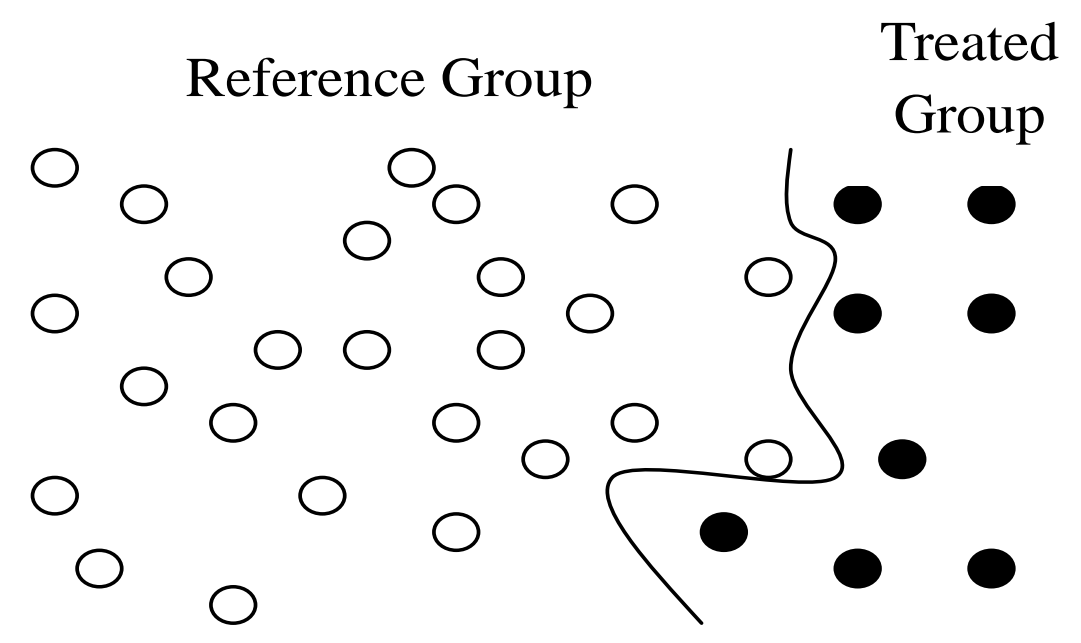

Figure 1.1 Reference and Treated Group

Existing before-after evaluation schemes identically follow a logical approach as demonstrated in Figure 1.2. 


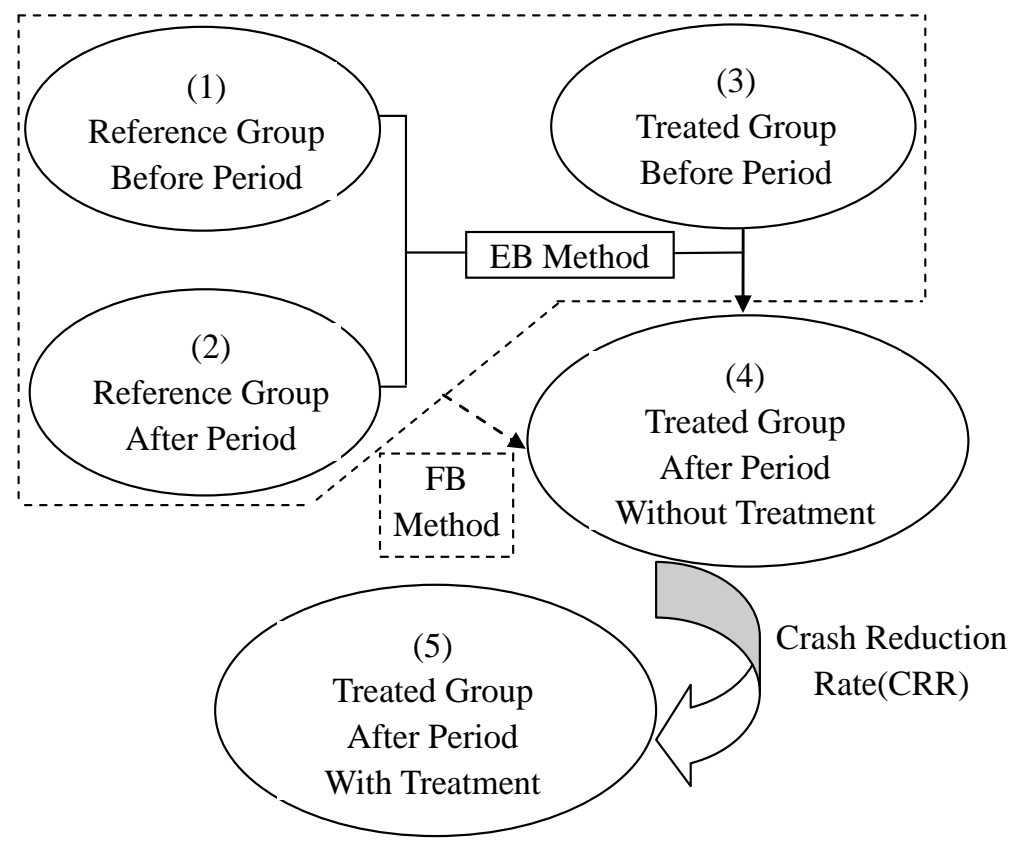

Figure 1.2 Logical Framework for Before-after Evaluations

The five groups as identified in Figure 1.2 form a grid with the dimension of reference and treated groups crossed by dimension of before and after periods. The goal here is to seek a crash reduction rate (CRR), no matter how it is mathematically expressed, through a safety comparison between groups 4 and 5 (Chen, 2013).

The Bayesian calculation is to combine prior and current information to derive an estimate for the expected safety improvement of the treatment that is being evaluated. The difference between $\mathrm{EB}$ and FB is solely that $\mathrm{EB}$ uses 2-step procedure to combine prior and current information whereas FB integrates the information into a one-step process (Chen, 2013)

\subsubsection{Cross-Sectional Studies}

CMFs or CMFunctions from cross-sectional studies are developed by comparing the safety of a group of sites with a feature with the safety of a group of sites without that feature. In practice, 
this comparison is accomplished through the use of regression models, also called crosssectional models (Carter et al, 2012). These cross-sectional models are also called safety performance functions (SPFs) or crash prediction models (CPMs). SPFs and CPMs are regression models that relate crash frequency with traffic volumes and other site characteristics. The coefficients of the variables from these equations are used to estimate the CMFs or the CMFunctions associated with a treatment.

Since accidents are counts (i.e., non-negative integers), the Poisson regression model has been proposed as an option for cross-sectional modelling. However, the Poisson model restricts the mean and variance to be equal. In crash analyses, it is very common for the variance to exceed the mean, and this phenomenon is called over-dispersion. Negative binomial (NB) models are able to account for over-dispersion and have become the most common way for estimating SPFs. Typically, the NB models are estimated through maximum likelihood methods using generalized linear modeling approach (Carter et al, 2012; Persaud et al, 2012; Washington et al, 2011 \& Saleem, 2012).

\subsection{The Role of Crash Modification Factors or Functions}

According to Hauer (2012), when a decision for implementation of an action is considered one needs to know what change in crash frequency it is likely to cause. The decision could be that of "illuminating a presently unlit stretch of road", "of reducing the legal blood alcohol content from 0.08 to $0.05 \mathrm{ml} / \mathrm{l}$ ", "of using a $350 \mathrm{~m}$ radius for a horizontal curve instead of a $300 \mathrm{~m}$ radius", etc. In each case, the comparison is of (at least) two courses of action to be denoted 
'a' and 'b'. In the above examples 'a' may stand for 'illuminate', 'reduce', and 'use $350 \mathrm{~m}$ radius', while 'b' stands for 'leave unlit', 'keep at 0.08 ', and 'use $300 \mathrm{~m}$ radius'.

The comparison is always of the expected target crash frequency with the action implemented, to be denoted by $\mu_{a}$, against the expected target crash frequency prevailing under identical conditions but without the action implemented, denoted by $\mu_{b}$. Comparison would be usually done using the ratio $\mu_{a} / \mu_{b}$. This ratio is the Crash Modification Factor or Function of implementing 'a' instead of ' $\mathrm{b}$ ' and will be denoted fully by $\theta(a, b)$ or, when the context is clear or general, as $\theta$ (Hauer, 2012).

$\theta(a, b)=\frac{\text { Expected }(\text { target }) \text { crashes with 'a' }}{\text { Expected (target) } \text { crashes in identical conditions but with }{ }^{\prime b \prime}}$

$\theta(a, b)=\frac{\mu_{a}}{\mu_{b}}$

According to the OECD report "Developing an International Framework for Crash Modification Functions" (OECD, 2012);

- Crash Modification Factor or Function always pertains to some action or measure and two possible future states (e.g. with illumination or without; with a $350 \mathrm{~m}$ radius or a $300 \mathrm{~m}$ one; with the legal blood alcohol content at 0.05 or at $0.08 \mathrm{~mL} / \mathrm{L}$; etc.)

- Crash Modification Factor or Function always pertains to target crashes of specific type and severity category (e.g. fatal crashes in a region for illumination, all injury crashes on the curve, crashes where alcohol was a causal factor for blood alcohol content limits, etc.) 
- Crash Modification Factor or Function depends on various details and circumstances. For example, the factor/function for the radius of horizontal curvature will depend on the two radii, on the approach speed, on the angle between the tangents, on whether the road is urban or rural, etc.

- The expected safety effect depends not only on the Crash Modification Factor or Function but also on the expected future number of target crashes, a quantity that is also imperfectly known.

\subsection{Current State of Crash Modification Factor/Function Research}

The following sections discuss issues with the current CMF/CMFunction research with a focus on inefficient decision making, transferability, and the nature of the CMF as a random variable.

\subsubsection{Inefficiency in Decision-Making}

CMF/CMFunctions are used to predict the safety effect of interventions, treatments, countermeasures, choice of design, choice of traffic control, etc. (Hauer, 2012). These predictions influence the decisions about whether to implement the action or not. The decision will be correct if the current mean CMF or CMFunction estimate corresponds sufficiently closely to the CMF or CMFunction that would materialize in the circumstances of a site if the contemplated action was implemented; if the two are not close the decision may be incorrect (Hauer, 2012). The Highway Safety Manual (2010) and the FHWA maintained Crash Modification Factor Clearinghouse Database do not provide information about how closely the two might correspond. As a result, one cannot know whether decisions to implement or not 
to implement some actions are very likely to be right, or whether a substantial proportion of such decisions will be wrong.

This issue is illustrated clearly in the OECD report "Developing an International Framework for Crash Modification Functions" (OECD, 2012), in the following text: "Assume that one has to decide whether to implement a certain countermeasure for which evaluation studies in the past have shown CMF estimates of 0.83, 0.63, 0.63, and 0.54 with the corresponding standard errors of $\pm 0.07, \pm 0.11, \pm 0.05$, and \pm 0.09 . On this basis one can say that the CMFs for this action or measure have a probability distribution with a (weighted) mean of about 0.66 and a standard deviation of about \pm 0.08 . This leads to an important insight about the key feature of the situation: while the decision whether to implement an action or measure is best based on the estimate of the expected CMF (0.66 in the example), the actual CMF will be most likely different. In this example it will be somewhere in the range $0.66 \pm 2 \times 0.08$, i.e., between 0.48 and 0.84. In short, the actual CMF, which will determine the outcome of a future action, is not the average CMF of past estimates".

From this one can observe that the actual CMF is not the average of all of the estimated CMFs. According to Hauer (2012), the decision to implement a countermeasure depends on the probability distribution of the CMF with a narrower distribution suggesting of a positive outcome as a result of the implementation. 


\subsubsection{Differences Between What Can be Observed and What Needs to be Known}

About Crash Modification Factors

According to Hauer (2012), if the CMF of some action is thought of as a constant that is estimated by the weighed mean of available estimates, then what matters is the standard error of that mean. However, if the CMF is considered a random variable then what matters for decision making is the standard deviation of its distribution. The standard error of the weighted mean can be reduced by replication. However, to reduce the standard deviation of the CMF distribution one has to make the CMF a function of the variables and circumstances that affect its magnitude. A correct view of CMFs is a prerequisite for productive research as Hauer (2012) concludes.

\subsubsection{Transferability of Crash Modification Factors/Functions}

Whenever some future action is contemplated, one must rely on CMF and CMFunction estimates that come from experience with similar actions implemented in the past and elsewhere. How well can one trust these to predict the potential safety effect of the future action now under consideration? Will the safety effect of Daytime Running Lights (DRL) in Italy be similar to what it was in Sweden? Will reducing the mean speed by a certain amount have the same effect in Europe and in North America? Will the paving shoulders on two lane roads in Ontario be the same as in Alabama? These are questions of 'transferability' noted in OECD (2012).

According to OECD (2012), one may reasonably expect that an action taken in identical circumstances will have identical results, but unfortunately, circumstances are never identical. 
Italy differs from Sweden, Europe from North America, and Ontario from Alabama. To what extent these differences matter is an empirical question as the OECD report notes in suggesting that if the previous research shows that the safety effect of DRL depends, among other things, on geographic latitude, it can act as a kind of empirical evidence that enables us to make use of results from Sweden, Finland or Canada and tailor it for application in Italy. The report further notes that the evidence of the research showing that reducing the mean speed by a certain amount had a similar effect on safety in Europe as in North America would allow one to pool and apply research results across jurisdictions.

In this sense, as noted in OECD (2012), the question of transferability amounts to asking to what extent the CMF of some action or measure depends on the circumstances that characterize a certain jurisdiction; and the greater our understanding of how CMF or CMFunctions depend on circumstances and variables, the less acute the question of transferability will become. Other sources, including Hauer (2012) and Elvik (2009 \& 2013) have also made similar arguments.

\subsubsection{Crash Modification Factor as a Random Variable}

The current practical framework of long-term expectancy of crashes is actually not directly from one single SPF equation. Instead, it is estimated by an algorithm that incorporates a base

SPF and several CMFs, as employed by the Highway Safety Manual (2010);

$N_{\text {predicted }}=N_{S P F_{x}} \times\left(C M F_{1_{x}} \times C M F_{2_{x}} \times \ldots \ldots \ldots \times C M F_{n_{x}}\right) \times C_{x}$

Where; 
$N_{\text {predicted }}=$ Predicted average crash frequency for a specific year for site type $x$,

$N_{S P F_{x}}=$ Predicted average crash frequency determined for base conditions of the SPF developed for site type $x$,

$C M F_{n_{x}}=$ Crash Modification Factor specific to the SPF for site type $x$ to adjust for non-base conditions, and

$C_{x}=$ Calibration factor to adjust SPF to local conditions for site type $x$.

As can be seen from the equation above, the crashes are not directly predicted by one unified function, but rather from a "block building process" in which the function is first used to estimate crashes with certain features of a situation called a base condition, then adjusted by multiple CMFs for non-base conditions, and finally calibrated by a local factor.

The major components of this model are CMFs among which each of them represents the safety impact from one specific design feature. Theoretically, the CMFs should be chosen on a sound basis of data and profound analyses, such as the base condition SPF itself, but facts are not always as such (Hauer, 2012).

Some CMFs are from a combination of observational data and subjective judgment by experts, while others are totally based on the knowledge of experts. That is, CMFs are highly empirical, but not very theoretically convincing (Hauer, 2012).

With regards to the format of CMFs, many are just given as a series of default values. Without a similar type of function used as the base condition SPF, the CMFs are still a source of considerable variance in Equation 1.4. 


\subsection{Issues with Current Research}

Based on the background information provided in this chapter, following are the key issues that this research addresses.

\subsubsection{Need for CMFunctions}

Based on the current state of CMF/CMFunction research provided in Section 1.4, it is clear that there is a need to make CMFs depend on the application circumstances and in order to do this we need to move away from the current single valued CMFs and make them a function of variables and circumstances that effect their magnitude. Thus by developing CMFunctions that relate the CMF to application circumstances, we will move towards making CMFs more transferable than CMFs that are averaged over application circumstances. This research explores the potential of cross-sectional data to convert the random valued CMFs into CMFunctions in a bid to make them more transferable and more representative of the application circumstances.

\subsubsection{Limitations of Before-After Studies}

As was mentioned before in Section 1.2, before-after studies are the preferred type when it comes to evaluating safety as they give us the ability to accurately predict the change in crashes as they would have materialized in real life. In order to get a CMFunction, data for a large number of before-after applications would be needed in order to reliably derive the link between the CMF and application circumstances. Such data are typically not available. In addition, for some cases, collecting before-after data for even a single-valued CMF is not possible. An example of such a case would be changing horizontal curvature of a road since 
there can literally be infinite combinations of curvature before and after a change. These limitations of before-after studies makes cross-sectional studies necessary.

\subsubsection{Issues with Cross-Sectional Regression}

Cross sectional regression has its own issues such as correlation of variables and omitted variable bias which can lead to counter-intuitive results since differences in safety implied by the regression effect estimated for a variable could be due to factors other than those evaluated. This research tries to answer two main questions in this regards The first being can we resolve these issues and the second being can we make cross-sectional studies work knowing that they are necessary. As part of this investigation the potential of cross-sectional studies to better estimate CMFs/CMFunctions even in the presence of before-after data is explored.

\subsection{Research Objectives}

The main purpose of this research is to advance the prediction methodology for estimating the safety impacts of highway design and operational elements. This research will look into answering two different research questions in order to achieve the main purpose. The first, and primary question relates to key issues identified in this chapter for the development of better approaches for estimating CMFs using both cross-sectional and before-after data in order to evaluate the potential of cross-sectional analysis for developing CMFs/CMFunctions. The other research question relates to developing new approaches for directly estimating safety at roundabouts through safety performance functions that use conflicting volumes and delay in order to determine whether the considerable extra effort required to collected the turning 
movement counts (which are needed to calculate the conflicting volumes and delay) would be worthwhile.

The main goals of this research can then be summarized as follows;

- To import prior research outcomes to obtain broader information,

- To convert random values of CMFs into CMFunctions to obtain better estimations,

- To evaluate the potential of cross-sectional analysis in the absence of before-after data and for better estimating CMFs/CMFunctions when before-after data are available,

- To evaluate the consistency in the estimation of CMFs by comparing the estimates from empirical Bayes before-after analysis and cross-sectional analysis using actual before-after data,

- To introduce new approaches to estimate safety of an entity, and,

- To integrate these outcomes together to achieve better approaches to estimate safety impacts.

\subsection{Research Design}

In order to meet the objectives defined above, this research is divided into two major components. The first component looks at evaluating the potential of cross-sectional analysis for developing CMFs/CMFunctions used to represent the effect of safety treatments on crashes in both the absence and presence of the data required for the preferred before-after methodology. This component is further divided into two parts. 
The first part looks into evaluating the potential of cross-sectional analysis in the absence of before-after data. Flattening of horizontal curves on rural two-lane roads will be used as a case study for this part. This main purpose of this evaluation is to provide new insights on estimating safety effects of horizontal curvature on rural two-lane highways, which would be impossible to evaluate with before-after data.

The second part looks into evaluating the consistency in the estimation of CMFs by comparing the estimates from empirical Bayes before-after analysis and cross-sectional analysis using actual before-after data. The design elements used in this part involve combination (center line plus shoulder rumble strips), wet reflective pavement markings in various road class categories and intersection conflict warning systems. The main purpose of this evaluation is to bridge the gap between cross-sectional and before-after analysis. This is done by conducting crosssectional analysis using a combination of untreated reference sites and after period data from treatment sites and comparing the results of this analysis to recent empirical Bayes before-after studies conducted using the datasets used in this investigation. The potential of cross-sectional analysis is further explored by developing CMFunctions to better capture the variation of the CMF with certain factors, something rarely achievable using before-after analysis.

The second component of this research looks into the use of turning movement counts to estimate the link between safety and traffic operations at roundabouts. Both single- and multilane roundabouts were used as design elements for this part. In doing so, new approaches incorporating the use of estimated conflicting volumes and overall vehicle delay (both of which could only be estimated using turning movement counts) at roundabouts will be introduced and this methodology will be evaluated against the traditional models based on traffic flow. 
The main purpose of this evaluation is to find consistency in evaluations from the new methods in order to determine whether it would be worthwhile to put that extra effort needed to collect turning movement counts and open the door for further research in this area.

The detailed strategy used to meet these objectives alongside a research plan with subobjectives are discussed in Chapter 3 of this dissertation.

\subsection{Dissertation Structure}

This dissertation consists of seven chapters. This first chapter gives a brief introduction about the current situation of road safety in Canada followed by the background and motivation behind the dissertation and the issues with current research. It also lists the objectives of this dissertation before this final section on the dissertation structure.

Chapter 2 focuses on reviewing current literature in crash modelling. It talks about different modelling techniques and the structure of collision models followed by a discussion on the need of cross-sectional regression models for estimating the CMFs/CMFunctions.

Chapter 3 focuses on the research design, plan and methodology. It discusses the research subobjectives and provides a flow chart of the research strategy. It further explains the research methodology and how crash prediction models were developed.

Chapters 4, 5, and 6 focus on the major components of this research as discussed in Section 1.7. These are standalone chapters in that each of them provides a brief literature review of the component in question, motivation behind choosing the component, a summary of data used 
for the investigation, and a detailed evaluation and comparison, followed by conclusions drawn from the investigation.

The first major component of this research is divided into two parts. Chapter 4 focuses on the first part: evaluating the potential of cross sectional analysis in the absence of before-after data. Chapter 5 focuses on the second part: evaluating the consistency in the estimation of CMFs from cross-sectional and before-after studies. Chapter 6 focuses on the second major component of this research: new approaches to estimating roundabout safety - case studies using single- and multi-lane roundabouts.

Lastly, Chapter 7 concludes this dissertation by providing a brief summary of accomplishments of this research, conclusions, and recommendations for future work. 


\section{Chapter 2. Review of Current Literature on Crash Modelling}

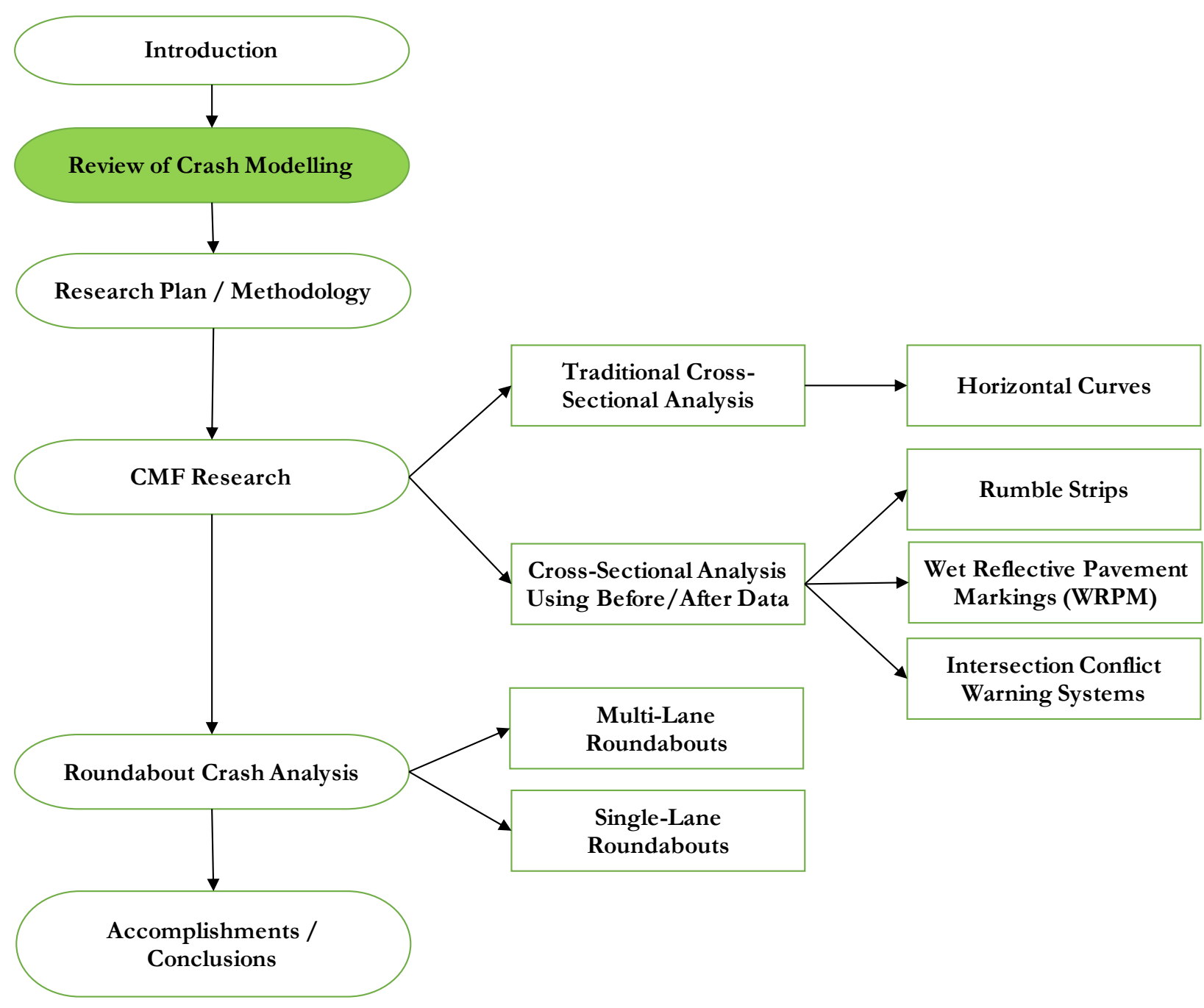

This chapter provides an in-depth review of current crash modelling techniques. It discusses various regression modelling approaches including generalized linear modelling and generalized estimating equations. It also discusses structural modelling with a focus on multilevel crash modelling. The last part of this section touches on the importance and need of crosssectional regression models for estimating the CMFs/CMFunctions. 


\subsection{Generalized Linear Models}

The concept of crash modelling to predict crashes has been supported by various researchers. Safety analysts use the crash prediction models to estimate the level of safety at different locations in order to identify the unsafe locations and the problems there that need to be addressed. In the past years, many crash prediction models have been developed by different researchers to evaluate the effects of different variables on safety. The most common crash prediction model uses the traffic volumes as the main explanatory variable to predict crashes. In one of the earlier efforts, Persaud and Dzbik (1993) used a generalized linear model that shows positive relationship between crash data and traffic flow. Many other variables can also be added to the models to take into account various other aspects and details about the infrastructure, vehicles and human behavior.

Elbasyouny (2011) classifies the methods of crash modelling into two main categories; conventional analysis and probabilistic analysis. Conventional analysis assumes that the observed crashes at a specific site can be considered as an unbiased estimate of the true level of safety at the site. Probabilistic analysis on the other hand defines the true crash frequency or any other parameter as a random variable with a probability distribution. Probabilistic methods account for the stochastic effect in crash data and recognize that crashes are rare random events and the mean crash frequency is never known (as in the conventional methods) but estimated. Thus, due to large statistical uncertainty and the failure to acknowledge the effect of various aspects, the conventional method is no longer favoured by researchers. 
Milton et al (2008) investigated the statistical properties of different regression models by using Poisson and Negative Binomial (NB) regression models instead of the linear regression model to estimate the crash frequency over a period of time. The effects of low sample mean values and small sample size on the estimation of the fixed dispersion parameter using NB model were also investigated in the study carried out by Lord (2006).

Various other researchers have also investigated the effects of a variety of other factors (other than the traffic volumes) on safety. For example, Shankar et al. (1996) investigated the relationship between crashes, weather and geometric features. The type and quality of pavement and the presence of parking and turning lanes were investigated by Matthew et al. (2002). Similarly, Park et al. (2010) investigated the relationship between speed differentials on highway segments using hierarchical linear regression models.

The most common approach used within the probabilistic approach to model crashes is Generalized Linear Regression Modelling. The GENMOD statement in SAS (SAS, 2016) is specifically designed for fitting generalized linear models. The GENMOD statement can be used to model data using a variety of probability distribution such as the Poisson, Negative Binomial (NB), Poisson Lognormal, and many others. The following sections discuss the most commonly used probability distributions in regression models.

\subsubsection{Poisson Regression Models}

The Poisson distribution is one of the most commonly used distribution to model discrete, nonnegative and random count data. It was one of the first distributions used to model crashes 
(Elbasyouny, 2011). Let $Y_{i}$ denote the number of crashes at site $i(i=1, \ldots \ldots, n)$ and assume that crashes at the $n$ sites are independent and follow;

$Y_{i} \mid \theta_{i} \sim \operatorname{Poisson}\left(\theta_{i}\right)$

The probability of a site $i$ having $y_{i}$ crashes is given by;

$\operatorname{Pr}\left\{Y_{i}=y_{i} \mid \theta_{i}\right\}=e^{-\theta_{i}} \theta_{i}^{y_{i}} / y_{i} !$

The Poisson parameter $\theta_{i}$ is commonly specified as an exponential function of site-specific attributes such as exposure, traffic and geometric characteristics usually expressed as;

$\theta_{i}=\exp \left(X_{i}^{\prime} \beta\right)$

Where;

$X_{i}^{\prime}=$ a row vector of covariates representing site-specific attributes, and

$\beta=$ a vector of regression parameters to be estimated from the data.

In the Poisson regression model, the mean $E\left(Y_{i}\right)$ and variance $\operatorname{Var}\left(Y_{i}\right)$ of the count variable are constrained to be equal. Various researchers such as Cameron and Trivedi (1998) and Winkelmann (2003) have shown that most crash data are generally likely to be over dispersed (i.e. the variance is greater than the mean) which makes the application of simple Poisson regression problematic, since by definition, the variance and mean are equal for this distribution. Assuming a Poisson distribution in the presence of over-dispersed data can underestimate the standard errors of the regression coefficients, which can lead to inflated 
values of $t$-test thereby affecting the significance level of the model regression coefficients. This will in turn lead to an incorrect selection of covariates resulting in faulty inference as well as affecting the goodness-of-fit.

\subsubsection{Negative Binomial (Poisson Gamma) Regression Models}

To overcome some of the problems associated with the Poisson regression several researchers proposed the use of the Poisson Gamma hierarchy leading to the Negative Binomial (NB) regression model. The NB regression model is an extension of the Poisson model developed to account for the over-dispersion that commonly exists in crash data (Elbasyouny, 2011).

To address over-dispersion, it is assumed that;

$\theta_{i}=\mu_{i} \exp \left(u_{i}\right)$

$\mu_{i}=\exp \left(X_{i}^{\prime} \beta\right)$

The term $\exp \left(u_{i}\right)$ represents a multiplicative random effect. The NB model is obtained by the assumption that;

$\exp \left(u_{i}\right) \mid \kappa \sim \operatorname{Gamma}(\kappa, \kappa)$

Where;

$\kappa=$ the inverse dispersion parameter.

The dispersion (or over-dispersion) parameter is sometimes referred to as $\alpha(=1 / \kappa)$. The probability density function of the NB model is given by; 
$\operatorname{Pr}\left\{Y_{i}=y_{i} \mid \mu_{i}, \kappa\right\}=\frac{\Gamma\left(y_{i}+\kappa\right)}{y_{i} ! \Gamma(\kappa)}\left(\frac{\kappa}{\kappa+\mu_{i}}\right)^{\kappa}\left(\frac{\mu_{i}}{\kappa+\mu_{i}}\right)^{y_{i}}$

The mean $E\left(Y_{i}\right)$ and variance $\operatorname{Var}\left(Y_{i}\right)$ under NB model are given by;

$E\left(Y_{i}\right)=\mu_{i}$

$\operatorname{Var}\left(Y_{i}\right)=\mu_{i}+\mu_{i}^{2} /_{\kappa}$

When crash data are not over-dispersed, $\alpha$ will tend to zero and the NB model will reduce to the Poisson model. It is therefore clear that the selection between the two models is dependent upon the value of $\alpha$. The NB regression model has been widely applied in the road safety literature. Arguably, the main reason for the extensive use of this model is that it is simple to compute since the gamma distribution is a conjugate prior to Poisson leading to a gamma posterior distribution which simplifies the posterior analysis considerably (Elbasyouny, 2011).

\subsubsection{Poisson Lognormal Regression Models}

Lord and Miranda-Moreno (2008) \& Aquero-Valverde and Jovanis (2008) have proposed the use of Poisson Lognormal (PLN) regression model as an alternative to NB models to model collision data. Like NB models, PLN models are also used to address the over-dispersion in the data and are obtained under the following assumption;

$\exp \left(u_{i}\right) \mid \sigma_{u}^{2} \sim \operatorname{Lognormal}\left(0, \sigma_{u}^{2}\right)$, or

$u_{i} \mid \sigma_{u}^{2} \sim \operatorname{Normal}\left(0, \sigma_{u}^{2}\right)$

Where; 
$\sigma_{u}^{2}$ denotes the extra Poisson variance.

In the NB model, $\exp \left(u_{i}\right)$ followed a Gamma distribution, whereas, in the PLN model, $\exp \left(u_{i}\right)$ follows a Lognormal distribution. The PLN is a good candidate for modeling crash occurrence in the presence of outliers, since its tails are known to be asymptotically heavier than those of the Gamma distribution.

The mean $E\left(Y_{i}\right)$ and variance $\operatorname{Var}\left(Y_{i}\right)$ under PLN model are given by;

$E\left(Y_{i}\right)=\mu_{i} \exp \left(0.5 \sigma_{u}^{2}\right)$

$\operatorname{Var}\left(Y_{i}\right)=E\left(Y_{i}\right)+E\left(Y_{i}\right)^{2}\left(\exp \left(\sigma_{u}^{2}\right)-1\right)$

A major limitation for the application of the PLN regression model is that its marginal distribution does not have a closed form as the NB model. As a result, the PLN has been less popular since it demands more computational effort and few statistical programs are readily available for their calibration. However, enormous progress has been made recently allowing for the application of the PLN model to analyze crash data.

\subsubsection{Zero Inflated Regression Models}

Crash data can have a high proportion of observed zero counts, particularly when analyzing crash occurrence in rural areas, and this is known to cause over-dispersion. According to Miranda-Moreno (2006), the issue is problematic when the number of observed zero counts exceed the zero counts that can be tolerated by the simple Poisson regression model. If a large number of sites have reported zero crashes for a given time period of observation this will 
produce a crash frequency distribution with heavy proportions of zeros. Such data sets are characterized by having a low sample mean problem (Miranda-Moreno, 2006).

Several studies tried to address the problem arising from the "excess" zeroes in crash data, by applying zero-inflated probability models such as zero-inflated Poisson (ZIP) and zero-inflated Negative Binomial (ZINB). The models assume that a dual-state process "safe" and "unsafe" is responsible for generating the collision data. The general consensus indicated an improved fit to data compared to the regular Poisson and NB models. Lord et al. (2005) criticized the dual-state approach and argued that "excess" zeroes arise from a combination of low exposure, high heterogeneity and sites categorized as high risk; inappropriate selection of time/space scales; under-reporting; and/or omitting important covariates. The authors recommended that a careful selection of the time/space scales for analysis, including an improved set of explanatory variables and/or unobserved heterogeneity effects in count regression models, or applying small area statistical methods (for data characterized by low exposure) represent the most defensible modeling approaches for datasets with a preponderance of zeros.

\subsection{Generalized Estimating Equations}

The reliability of crash prediction models is enhanced by using as many years of data as possible. This could be enhanced further by accounting for the variation/trend in the year to year crash counts because of the influence of factors that change every year (Lord et al., 2000).

Diggle et al. (1994) proposed three methods to estimate the coefficients of the crash prediction models accounting for the year to year tend in crash data: marginal models, transition models and random effect models. Mountain et al. (1998) and Maher et al. (1996) suggested avoiding 
the modelling of year to year crash variation due to the difficulties faced in handling temporal correlation. According to Lord et al. (2000), the multinomial maximum-likelihood function to incorporate the trend, as proposed by Hauer (1994), is too complicated and requires various mathematical manipulations that might be out of reach of an average modeller. Lord et al. (2000) concluded that most approaches available to model trend in crash data suffer from many limitations such as ignoring the temporal correlation, the model type may not always be appropriate for crash predictions, and that incorporating trend maybe too complicated for an average modeller.

In order to overcome these difficulties, Lord et al. (2000) in their paper illustrated the application of the generalized estimating equations (GEE) procedure to safety studies where several years of crash data is available and it is desirable to incorporate the year to year trend in crashes.

The GEE procedure as illustrated by Lord et al. (2000) can be modelled in SAS (SAS, 2016) using the GENMOD statement by defining the repeated subject (which in safety modelling should be the years in the crash data) and the correlation and the covariance matrices to account for the repeated observations.

The temporal correlation in the repeated observations can be described by a $n_{i} \times n_{i}$ matrix $R(\lambda)$, where $\lambda$ represents the type of correlation with $\lambda=\left[\lambda_{1}, \ldots \ldots, \lambda_{n-1}\right]$. In this formulation, $n_{i}$ is the number of subjects or in easier words the number of years of crash data available. Thus, the new covariance matrix now becomes;

$V_{i}=A_{i}^{0.5} R_{i}(\lambda) A_{i}^{0.5}$ 
Where,

$A_{i}$ is the an $n_{i} \times n_{i}$ matrix with diagonal $\left[V\left(\mu_{i 1}, \ldots \ldots \ldots, V\left(\mu_{i T)}\right]\right.\right.$

The covariance matrix is thus given by;

$\operatorname{cov}(\hat{\beta})=\sigma^{2}\left[\sum_{i=1}^{1} D_{i}^{\prime} V_{i}^{-1} D_{i}\right]^{-1}$

Where,

$\beta=$ Coefficient to be estimated, and

$D_{i}=\delta \mu / \delta \beta$

In order solve the GEE correctly, every element of the correlation matrix $R_{i}$ has to be known. In the case of repeated observations, it is not possible to know the proper correlation type for these observations. Hence Lord et al. (2000) in their paper propose the use of a working matrix $\widehat{V}$ of the correlation matrix $V_{i}$ which is based on the correlation matrix $\hat{R}_{i}$. The covariance matrix thus then becomes;

$\operatorname{cov}(\hat{\beta})=\sigma^{2}\left[\sum_{i=1}^{1} D_{i}{ }^{\prime} V_{i}^{-1} D_{i}\right]^{-1}\left[\sum_{i=1}^{1} D_{i}{ }^{\prime} \widehat{V}_{i}^{-1} V_{i} \widehat{V}_{i}{ }^{-1} D_{i}\right]^{-1}\left[\sum_{i=1}^{1} D_{i}{ }^{\prime} V_{i}{ }^{-1} D_{i}\right]^{-1}$

The proposed methodology by Lord et al. (2000) as shown above possess a very useful property in that $\hat{\beta}$ nearly always provides consistent estimates of $\beta$ even if the matrix $V_{i}$ has been estimated improperly. Thus the confidence interval of $\beta$ will be correct even when the covariance matrix is specified incorrectly. 


\section{$\underline{2.3 \text { Safety Performance Functions }}$}

The "Future Directions Subcommittee of the Task Force for the Development of a Highway Safety Manual" gave a series of potential directions on road safety analyses (TRB, 2009). From the Task Force's perspective, the most promising developments with regards to road safety models for the future include areas such as "structural modeling", "surrogate measures of safety" and "multi-scale safety and driver behavior modeling approach".

Other recent publications similarly embed more causal factors or real-time operational factors into safety performance functions (SPFs). Lee et al. (2003) researched "real-time" crash prediction models (CPM) and applied that model towards crash prevention in freeway traffic. In this model, crash precursors based on real-time traffic measures are used to quantify crash potential. The authors recommend the following function:

Crash frequency $=$ $f$ (crash precursors, external control factors, exposure)

Three crash precursors represent traffic flow conditions before crash occurrence: (a) the average variation of speed on each lane, (b) the average variation of speed difference across adjacent lanes, and (c) traffic density.

In another paper, Lee et al (2004) researched the impact of speed limits on road safety. However, driving behavior drew more attention from road safety modelers. Verschuur and Hurts (2008) tried to model safe and unsafe behaviors in their paper. Many other recent efforts on SPFs have tried new statistical approaches in the road safety domain. 


\subsection{Structural Modeling}

Traditional statistical approaches, such as the single-level generalized linear model (GLM) that form most of current well-established safety performance functions (SPFs), assume each observation (e.g. a crash or a vehicle involvement) in the estimation procedure corresponds to an individual situation. Hence, the residuals from the model exhibit independence. As a result, a typical structure of the GLM may be expressed as a general form, as introduced by Chin and Huang (2008):

$Y \mid \theta \sim \operatorname{Dist}(\theta)$

$\theta=f(X, \beta, \varepsilon)$

Where;

$Y=$ the dependent variable of interest, e.g., crash frequency, severity levels,

$\operatorname{Dist}(\theta)=$ the adapted distribution and its parameters,

$X=$ the covariates which represent various risk factors in crash occurrences,

$\beta=$ the factor effects of $X$ on $Y$,

$f()=$. the link function that relates $X$ and $Y$, and

$\varepsilon=$ the error/disturbance terms in the model.

In a single-level GLM, every element of array of $\beta$ is fixed i.e. expressed by point estimations, along with $\varepsilon$ as "iid" (independent and identical distribution) based on the assumption of 
homogeneity upon situations of crash occasions. Any heterogeneity of situation along spatial and time units is ignored. Such assumption may often not hold true since multilevel data structures extensively exist in traffic data. For crash studies, different road segments and different time periods occasionally lead to substantial "between-group heterogeneities" which break the ground of the conventional models with fixed parameters and "iid" assumed disturbance terms.

Many road safety researchers/modellers are recognizing that road safety data is naturally multilevel constructed (Chen, 2013). As a result, more efforts are being to develop models that address multiple structures. Such models include artificial intelligent models (AI), such as neural network $(\mathrm{NN})$ or Bayesian NN to model multilevel data structure; however, the shortages of these approaches make them incapable of generating explicit functional relationships and statistically interpretable results (Chen, 2013).

Another technique that addresses multiple structures involves the use of generalized estimating equations (GEE) as an extension of the GLM. When dealing with multilevel data structures, GEE aims to provide estimates with acceptable properties only for the fixed parameters in the model, while treating the existence of any random parameters as a necessary "nuisance". Hence, GEE may merely be superior in cases where the exact form of the multilevel data structure is unknown. The next way to distinctly address a multilevel data structure is to use hierarchical models (Chen, 2013).

The hierarchical model, also called a multilevel or random effect model, is a statistical technique that allows multilevel data structures to be properly specified and estimated. Chin 
and Huang (2008) in their paper give an example of a two-level hierarchical GLM road safety model. The typical structure of GLM is still effective for a hierarchical model. However, within a hierarchical model, the covariate vector $X$ is divided into three components, $c\left(1, X^{L 1}, X^{L 2}\right)$ to represent the factors associated with the intercept, level 1 (individual level) and level 2 (group level), respectively. Correspondingly, $\beta$ and $\varepsilon$ are also divided into different components to serve different functions. Hence, the link function becomes a combination of the models in terms of the two levels as shown below;

Level 1 Model: $f^{-1}(\theta)=\beta_{0}^{L 1}+X^{L 1} \beta^{L 1}+\varepsilon^{L 1}$

Level 2 Model: $\beta_{0}^{L 1}=\beta_{00}^{L 2}+X^{L 2} \beta_{0}^{L 2}+\varepsilon_{0}^{L 2}$

Level 2 Model: $\beta^{L 1}=\beta_{01}^{L 2}+X^{L 2} \beta_{1}^{L 2}+\varepsilon_{1}^{L 2}$

The combined model is obtained by substituting the level 2 models into the level 1 model;

$f^{-1}(\theta)=\left(\beta_{00}^{L 2}+X^{L 1} \beta_{01}^{L 2}+X^{L 2} \beta_{0}^{L 2}+X^{L 1} X^{L 2} \beta_{1}^{L 2}\right)+\left(\varepsilon^{L 1}+\varepsilon_{0}^{L 2}+X^{L 1} \varepsilon_{1}^{L 2}\right)$

As can be seen from the combined model, the link function now consists of a fixed part and a random part. The fixed part represents a deterministic relationship which is fully dependent on covariate $X$, while the random part is stochastically determined by the number of disturbance terms.

Lee et al. (2008) and Davis (2004) have also tried structural modeling or similarly complicated approaches in road safety. This fashion demonstrates the potential of structural or relevant hierarchical modeling in the area of road safety. 


\subsubsection{Multi-Level Crash Modelling}

The conventional types of crash models are baseline models (also known as the general AADT models) and models with covariates (also known as Full models) (Lord et al, 2008b). Baseline models are developed with AADT as the only variable (see Equation 2 -24) assuming general conditions of other potential variables. Full models (see Equation 2-25) on the other hand functionally include other covariates as a part of the SPF equation (Chen, 2013).

$$
\begin{aligned}
& N_{\text {predicted }}=\exp \left(\alpha+\beta_{1} \times \ln (A A D T)\right) \\
& N_{\text {predicted }}=\exp \left(\alpha+\beta_{1} \times \ln (A A D T)+\beta_{2} \times \ln \left(x_{1}\right)+\beta_{3} \times \ln \left(x_{2}\right)\right)
\end{aligned}
$$

Where;

$N_{\text {predicted }}=$ the predicted target crash frequency,

$\alpha \& \beta=$ the estimated parameter coefficients,

$x=$ the covariates, and

$A A D T=$ the average annual daily traffic.

Since the development of full models is still at the exploratory stages, the Highway Safety Manual (2010) did not propose its use in their predictive methodology (Chen, 2013 \& AASTHO, 2010). Instead, the Highway Safety Manual (2010) recommended crash prediction algorithms with base model predictions multiplied by CMFs (AASTHO, 2010). This has already been identified as an issue in Section 1.4 of this dissertation. 
The full model shown in Equation 2-22 can be functionally restructured into a multi-level model using a combination of a base SPF and sub-level CMFunctions, as shown in Equation 2-23. In this case, the base SPF will only be an AADT only SPF, whereas, the sub-level models will define the various coefficients that effect the safety of the entity in question (Chen, 2013).

First Level SPF: $N_{\text {predicted }}=\exp (A+B \times \ln (A A D T))$

Sub-Level CMFunctions:

$A=\alpha \times \exp \left(\left(\alpha_{1} \times x_{1}\right)+\left(\alpha_{2} \times x_{2}\right)+\left(\alpha_{n} \times x_{n}\right)\right)$

$B=\beta_{0} \times \exp \left(\beta_{1} \times\right.$ Local Factor $)$

Where;

$A \& B=$ parameter coefficient estimates from the first-level SPF,

$\alpha, \beta \& x=$ covariate coefficients estimated in CMFunctions, and

Local Factor $=$ the classification variable used when data from different jurisdiction is used .

Chen (2013) found multi-level models perform well under different scenarios and maintained a consistent model form after several iterations of re-sampling and re-estimating. Chen (2013) also found that the structural distinction of the covariates gave rise to deeper insights for safety impact mechanisms and also emphasized the nature of hierarchy for safety datasets. 


\subsection{Cross-Sectional Regression to Estimate CMFs/CMFunctions}

As discussed in Chapter 1, CMFs derived from before-after studies are estimated by the change in number of crashes due to the application of the treatment. Before-after studies are the preferred study type but they have their own issues, which may lead to inaccurate results. Some of these issues include sample size, regression to the mean effect, site selection bias, and mixed safety effects (Wu et al., 2014). Various other researchers including Gross et al. (2011), Hauer et al. (1983), and Lord et al. (2012) have talked about these issues, and concluded that due to the nature of data available for road safety studies these issues are bound to exist.

With an increased focus these days on developing CMFunctions rather than single valued CMFs (also one of the objectives of this research), before-after studies though preferred, do not provide an easy to use solution to derive CMFunctions (Wu et al, 2014). In order to do so, one would require a large number of before-after applications for various different application circumstances of a countermeasure that may happen in real life. Getting these data is always not possible and as such cross-sectional regression analysis becomes necessary for developing CMFunctions due to the ease of modelling interactions between the countermeasure and other road features even with limited data availability as was previously discussed in Section 1.5.

Wu et al. (2014) in their study worked towards better understanding the CMFs derived from cross-sectional studies and concluded that "the CMFs derived from cross-sectional regression models should be unbiased when all factors associated with the crash risk are identical in all segments, except the factors of interest", "the derived CMFs are still reliable even if some 
factors having minor safety effects are omitted in the models", and "the derived CMFs become unreliable when the factors known to have an effect on the crash risk are ignored or omitted."

Wu et al. (2014) emphasized the need for comparison between the CMFs derived from crosssectional studies and before-after studies, since, no study in the past have looked into investigating whether CMFs derived from cross-sectional regression models reflect the true safety effects of corresponding treatments.

\subsection{Chapter Summary}

This chapter provided an in-depth review of various crash modelling techniques. It talked about generalized linear modelling and the various probability distributions that are used in crash modelling. The discussion then moved towards the use of generalized estimating equations to account for the time trend and temporal correlation in the crash data. This was then followed by a discussion on structural modelling with a focus on multi-level crash models as they are needed to model CMFs/CMFunctions. The last part of this chapter further discussed the use of cross-sectional analysis to derive CMFs/CMFunctions and why cross-sectional analysis is necessary due to the nature of data available for safety studies. 


\section{Chapter 3. Research Design, Plan, and Methodology}

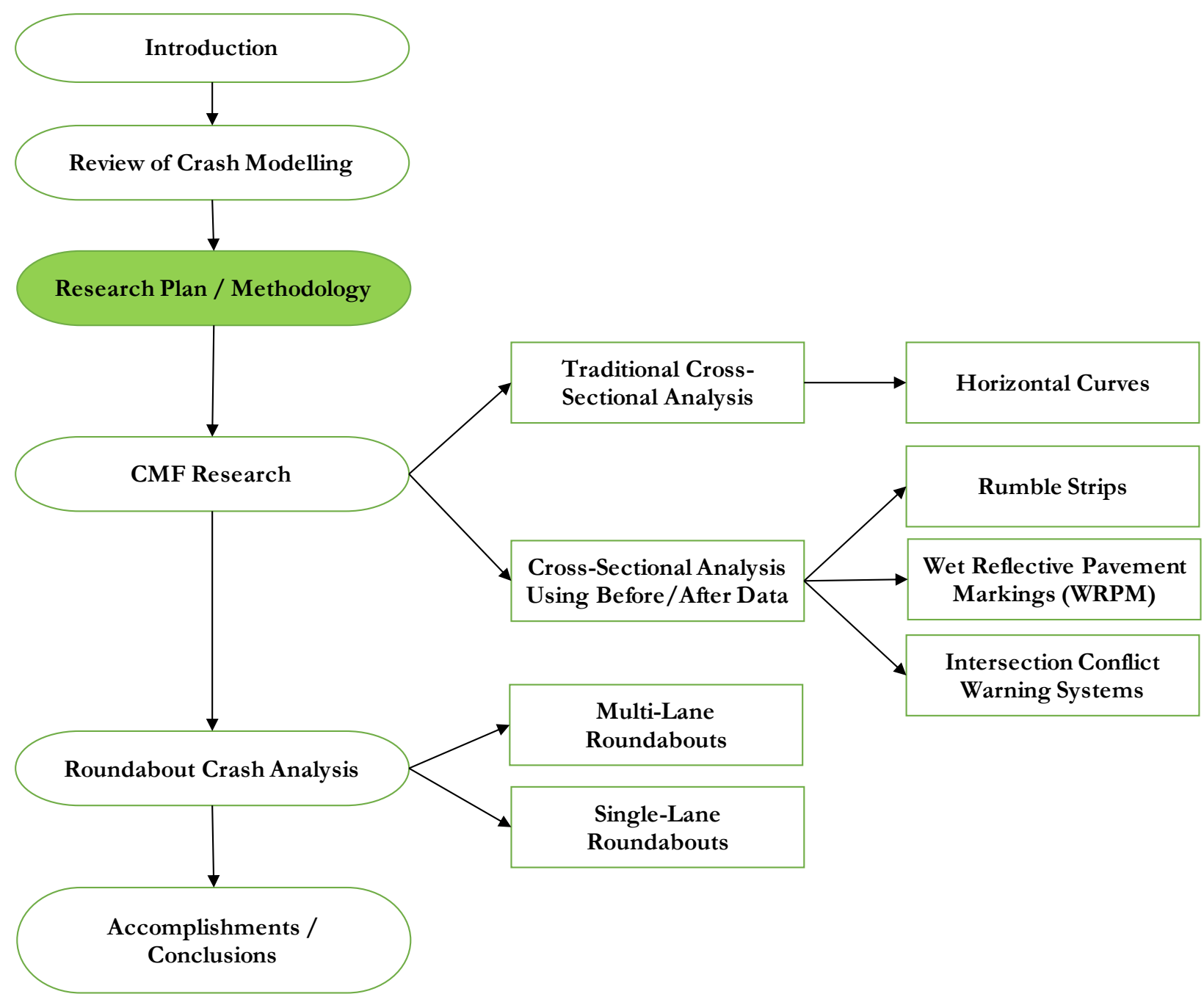

This chapter classifies the research's sub-objectives and lays out an expanded research strategy

(building on the main research objectives and strategy presented in Sections 1.6 and 1.7). This is then followed by the research methodology and allocation of tasks to achieve the goals of this research. 


\section{$\underline{\text { 3.1 Classification of Research Sub-Objectives }}$}

The fundamental goal of this dissertation is to advance the prediction methodology for estimating the safety impacts of highway design and operational elements. The essential strategy to realize this goal is to find the missing pieces within the current approaches (used to estimate safety impacts) using broader information obtained through prior research outcomes. By following this basic idea, the sub-objectives which serve the fundamental goal comprise of four aspects: comparing and assembling information from existing models; conversion of random values of CMFs into CMFunctions; improve transferability of the CMFs and CMFunctions to different jurisdictions; and improve the baseline models for roundabouts by using variables other than the approach AADTs. Figure 3.1 illustrates the classification of these research objectives.

Each of these sub-objective leads to answering key missing pieces that this dissertation will try to address. The key missing pieces include improving the reliability of CMFs and CMFunctions, eliminating the current arbitrarily defined factors, improving the transferability of CMFunctions, and developing new approaches to estimate safety of an entity.

\subsection{Design of Research Strategy}

Figure 3.2 demonstrates the research strategy that will help achieve the four major subobjectives as mentioned in Section 3.1. This strategy flows along an essential logic that outlines the process by following procedures on how to realize all parts of study and how to connect these parts as a whole. 


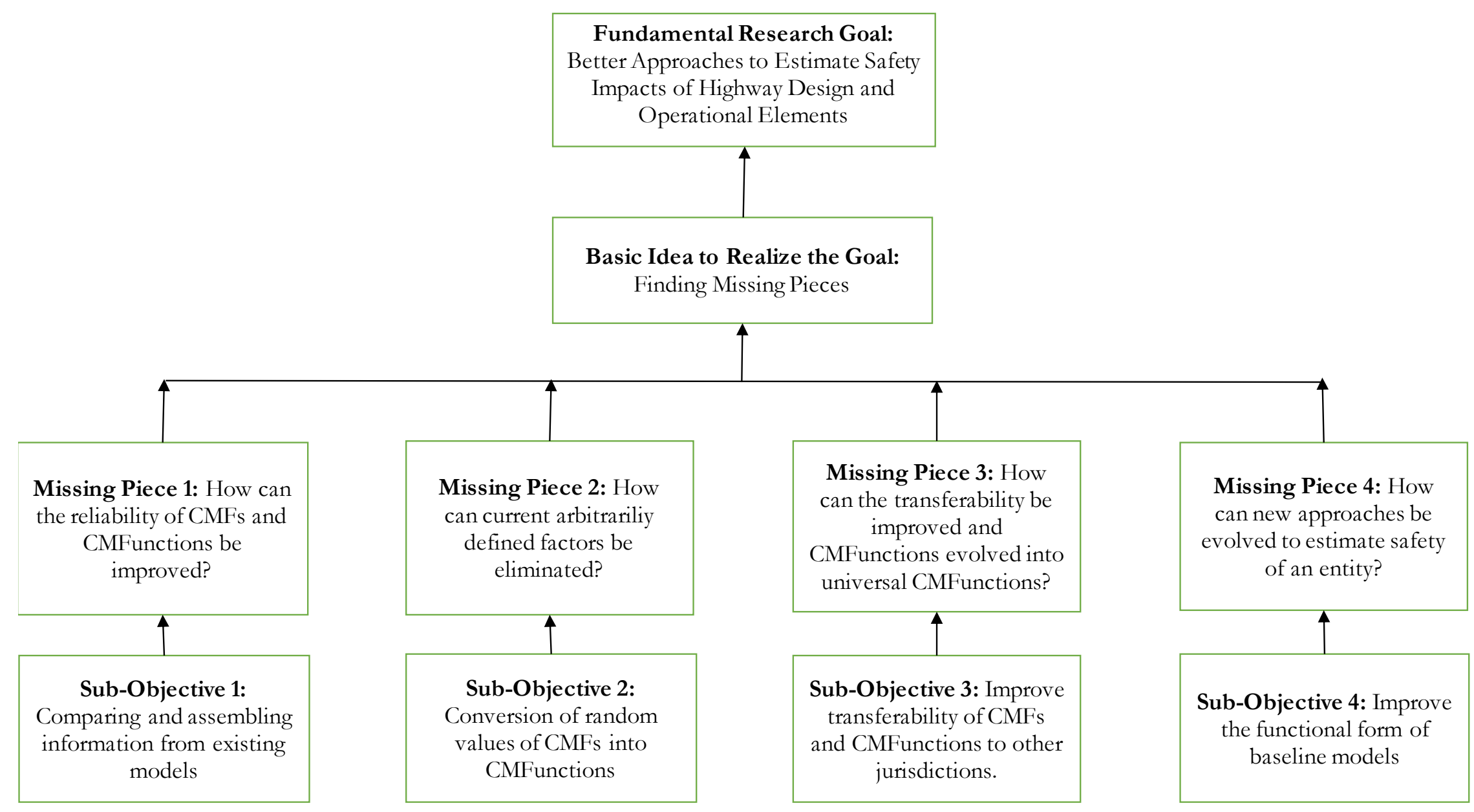

Figure 3.1 Classification of Research Sub-Objectives 


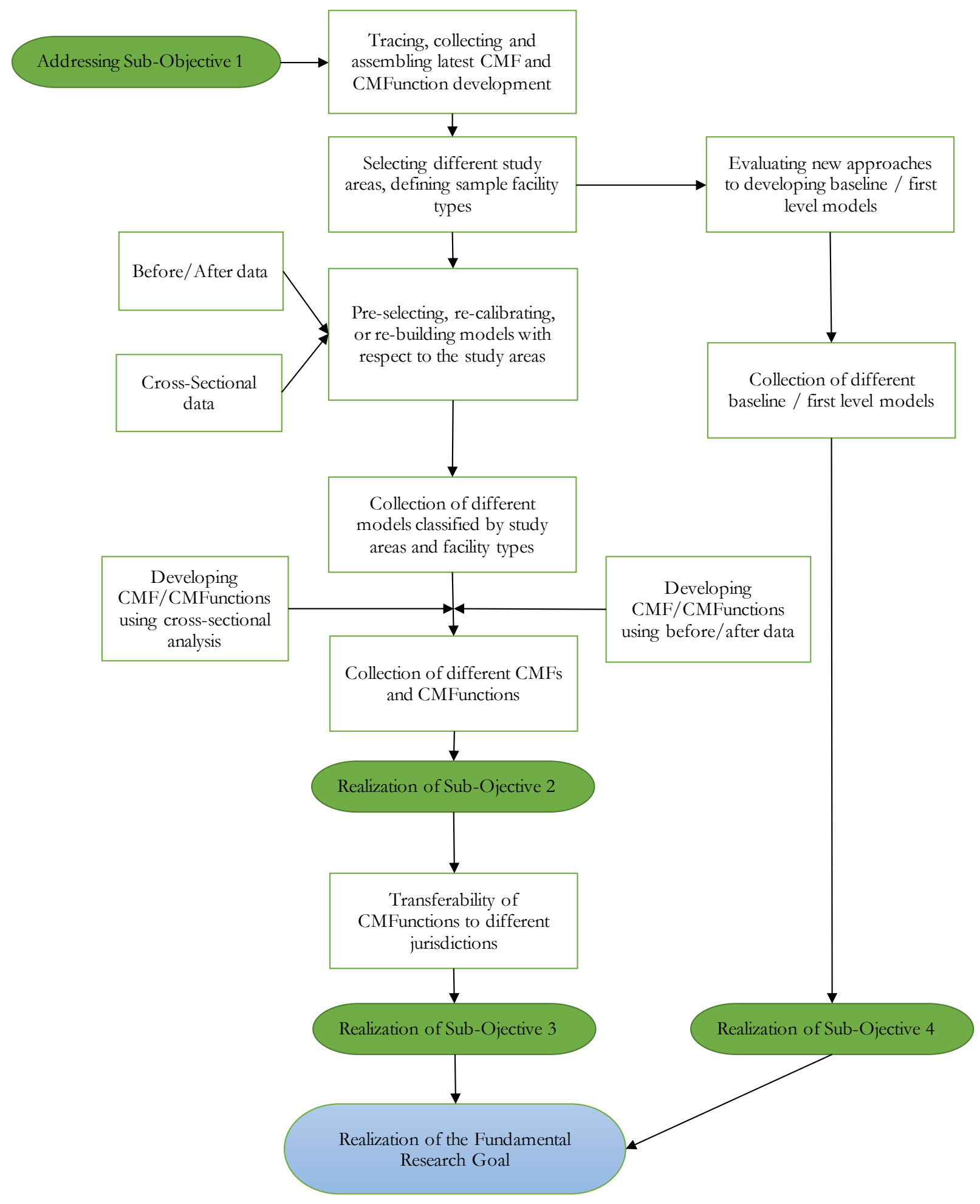

Figure 3.2 Research Strategy 


\section{$\underline{3.3 \text { Methodology }}$}

\subsubsection{Basic Philosophy}

The principal purpose of this dissertation is to study and improve the methodology for estimating safety impacts of different highway design and operational elements through exploring the development of new models and in some cases the improvement of already developed models. This philosophy governs the main ideas throughout this research.

The majority of studies in the road safety area, especially ones conducted for theses/dissertations, are overwhelmingly dominated with research and development of models, based on representative sample data and breakthrough statistical methods to assure "more excellent" or "better" results than other research counterparts. However, each of these results is purely a "sampling", which reflects an isolated piece of knowledge. The drawback to this kind of approach has been proven by different transferability studies, whereby, the model works good only when used within the sample it was developed from.

Correspondingly, this research will,

- Advance the use of CMFunctions as an effective way to (i) evaluate the safety impacts of proposed/implemented actions, and (ii) transfer models between different jurisdictions.

- Advance the assembly of models incorporating new approaches to estimate the safety of an entity. 


\subsubsection{Study Area and Sample}

The study areas used for this dissertation are vast and consist of both rural and urban sites with details about the many different features of the roadway. Different facility types that exist within the data are 2-lane highways, multi-lane highways, intersections on rural highway sections, etc.

The data are available for various states/provinces in both the U.S. and Canada. The characteristics of data are discussed in further detail in the next sections.

\section{$\underline{\text { 3.3.3 Allocation of Tasks }}$}

To determine an applicable plan, the research objectives were allocated further into different executable tasks.

\subsubsection{Tracing and Collecting the Latest Developments of CMFs and}

\section{CMFunctions}

The research and development of new approaches are being done on a continuous basis. Thus, this research requires an extensive and current collection of the CMFs and CMFunctions from all available sources.

\subsubsection{Data Collection}

This task consists of two subtasks. The first one is to get up to date data from the relevant jurisdictions, and the second one is to filter the data and make appropriate samples (with enough crashes and data points) for modelling. 


\subsubsection{Constructing a General SPF and CMFunction Configuration}

Regardless of the type of entities being modelled to predict crashes and the different impacts being evaluated by the use of CMFunctions, all relevant models will share similar SPF and CMFunction configurations. This general configuration accommodates all potential candidate models used in this research.

\subsubsection{Re-Calibrating Current Models to Convert CMFs to CMFunctions}

This task primarily focuses on the re-calibration of current models to convert the current arbitrary values of CMFs into CMFunctions. This task uses the current CMFs surveyed in Task 1 (these can be found in Chapter 4 and 5 for their respective treatments in question).

\subsubsection{Using Cross-Sectional Data and Before-After Data to Generate CMFs and CMFunctions}

Once the candidate models were selected and modelled, traditional cross-sectional analysis and cross-sectional analysis using before-after data was used to generate new CMFs and CMFunctions.

\subsubsection{Developing New Approaches to Estimate Safety of an Entity}

This task consists of developing new approaches to estimate safety of an entity by improving the functional form of the baseline models. The application is to roundabouts. 


\section{$\underline{\text { 3.4 Crash Prediction Models }}$}

Consistent with state-of-the-art methods, generalized linear modelling (GLM) with the specification of a negative binomial (NB) error structure, was used to develop the Crash Prediction Models (Persaud et al, 2012). In turn, the specification of an NB error structure allows for the direct estimation of the over dispersion parameter (one of the parameters used to assess the models) since this is a parameter of the NB distribution. As noted earlier, over dispersion occurs when the data have larger variance than what is expected under the assumption of a Poisson distribution.

Different models were developed linking different crash types to their relevant covariates. The general form of the most commonly used crash prediction model is as follows (Persaud et al, 2012):

Crashes $=e^{\alpha} \times$ Variable $1^{\beta_{1}} \times$ Variable $2^{\beta_{2}} \times---\times$ Years

Where;

Crashes $=$ Type of crash modeled (e.g. Total, Injury, Rear End, etc.),

$\alpha, \beta_{1}, \beta_{2}$, etc. $=$ Estimates parameter coefficients, and

Years $=$ No. of years of crash data used.

Several goodness-of-prediction measures were used to assess the predictive capabilities of each model. These include: 
- Plots of the cumulative residuals (CURE plots) - Observed minus predicted crash frequencies) graphed versus each variable in the model.

- Mean absolute deviation (MAD) - Absolute value of sum of observed minus predicted crash frequencies divided by sample size. Values of MAD close to zero indicate better predictions by the model.

- Mean squared prediction error (MSPE) - Sum of squared differences between observed and predicted crash frequencies divided by sample size.

- Mean prediction error (MPE) - Square root of the sum of squared differences between observed and predicted crash frequencies divided by sample size.

- Over dispersion parameter $(\mathrm{K})$ - Occurs when the data have larger variance than what is expected under the assumption of a Poisson distribution. Small values of K identify that the model better captures the over dispersion in the data.

Prior to assessing the goodness-of-fit of the models the calibration factors were derived. Because the model fitting process is actually fitting the logs of crashes and the independent variables, the sums of observed and predicted crashes are never exactly equal. Although the differences are small, in order to make equal comparisons between models, these differences were eliminated by applying calibration factors to each model as a multiplicative factor. The calibration factor is derived by dividing the sum of observed crashes by the sum of predicted crashes. 
It is important to not only evaluate a model based on overall measures but also to evaluate how it performs over the range of covariates. This evaluation makes use of cumulative residual (CURE) plots (See Figure 3.3) (Hauer and Bamfo, 1997 \& Hauer, 2014).

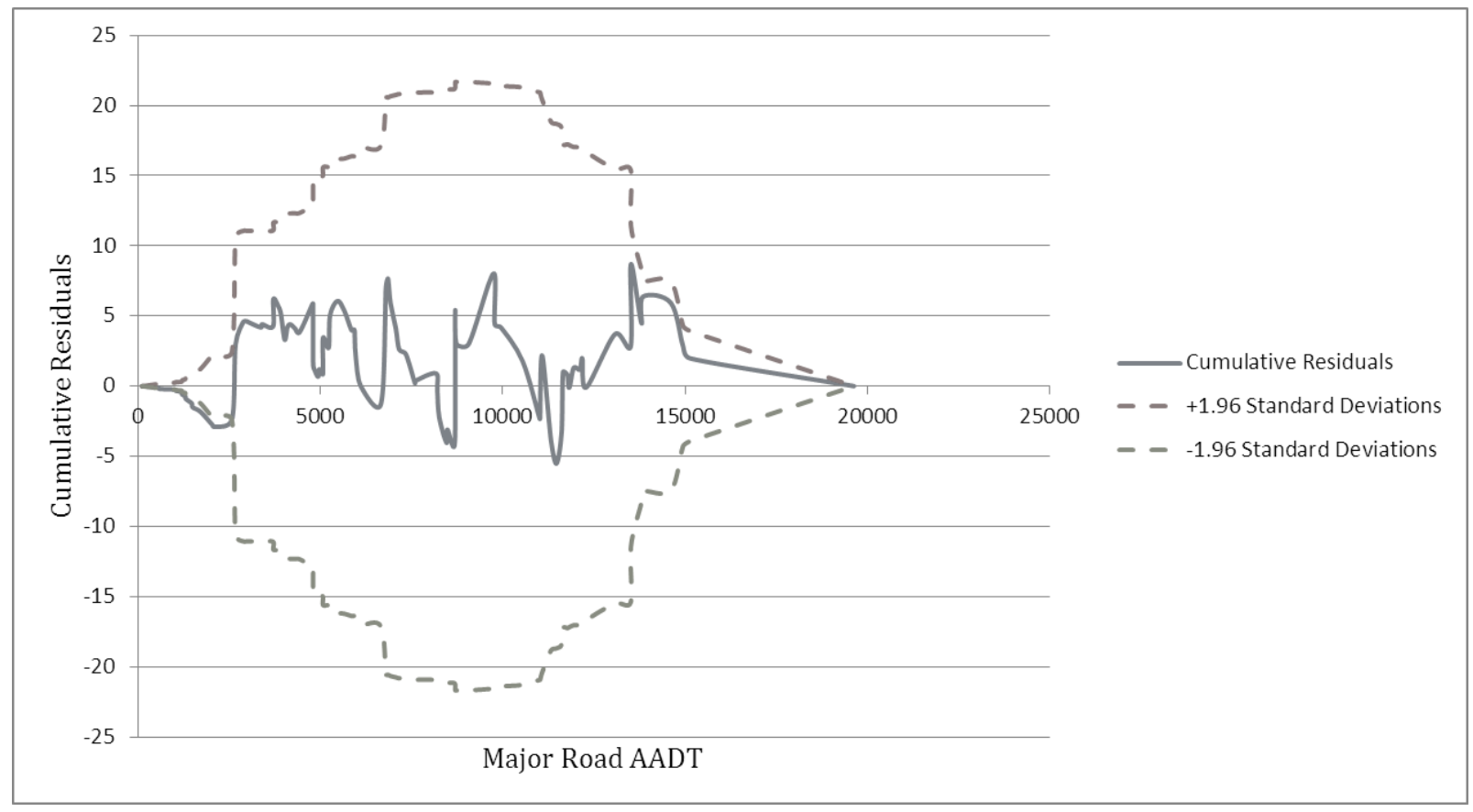

Figure 3.3 Example of a CURE Plot

In the Cumulative Residuals (CURE) method the cumulative residuals (the difference between the observed and predicted values for each site) are plotted in increasing order for each covariate separately. Also plotted are graphs of the $95 \%$ confidence limits. According to Hauer (2014), "the plots of cumulative residuals should not have vertical drops because these are indicative of inordinately large residuals - possible outliers. The plots should also not have long increasing or decreasing runs because they correspond to regions of consistent over and underestimation". If there is no bias in the model, the plot of cumulative residuals should oscillate without systematic over or under-prediction, and stay inside of these confidence limits 
(Hauer, 2014). The graph shows how well the model fits the data with respect to each individual covariate.

Figure 3.3 illustrates an example of the CURE plot for the Major Road AADT covariate. The indication is that the fit is very good for this covariate in that the cumulative residuals oscillate around the value of zero and lie between the two standard deviation boundaries.

Apart from using generalized linear modeling (GLM) with the specification of a negative binomial (NB) error structure, generalized estimating equations (GEE) were also applied in the second component of this research to account for time trend and/or temporal correlation in yearly crash data at roundabouts.

\subsection{Chapter Summary}

This chapter starts with disintegrating the key objectives of this research as identified in Section 1.6 into key sub-objectives, each of which lead to answering missing pieces in this research. This is then followed by a pictorial representation in form of flowcharts of the subobjectives leading towards the ultimate research goal and detailed research strategy (building upon material presented in Section 1.7), followed by the research methodology consisting of the basic research philosophy and the allocation of tasks. The last section of this chapter discussed the structure of the crash prediction models that will be used to develop models in the upcoming chapters and the goodness of prediction criteria that will be used to assess these models. 


\section{Chapter 4. Evaluating the Potential of Cross-Sectional}

\section{Analysis for Developing CMFs in the Absence of Before-After}

\section{Data - Case Study on the Safety Effects of Horizontal}

\section{Curvature}

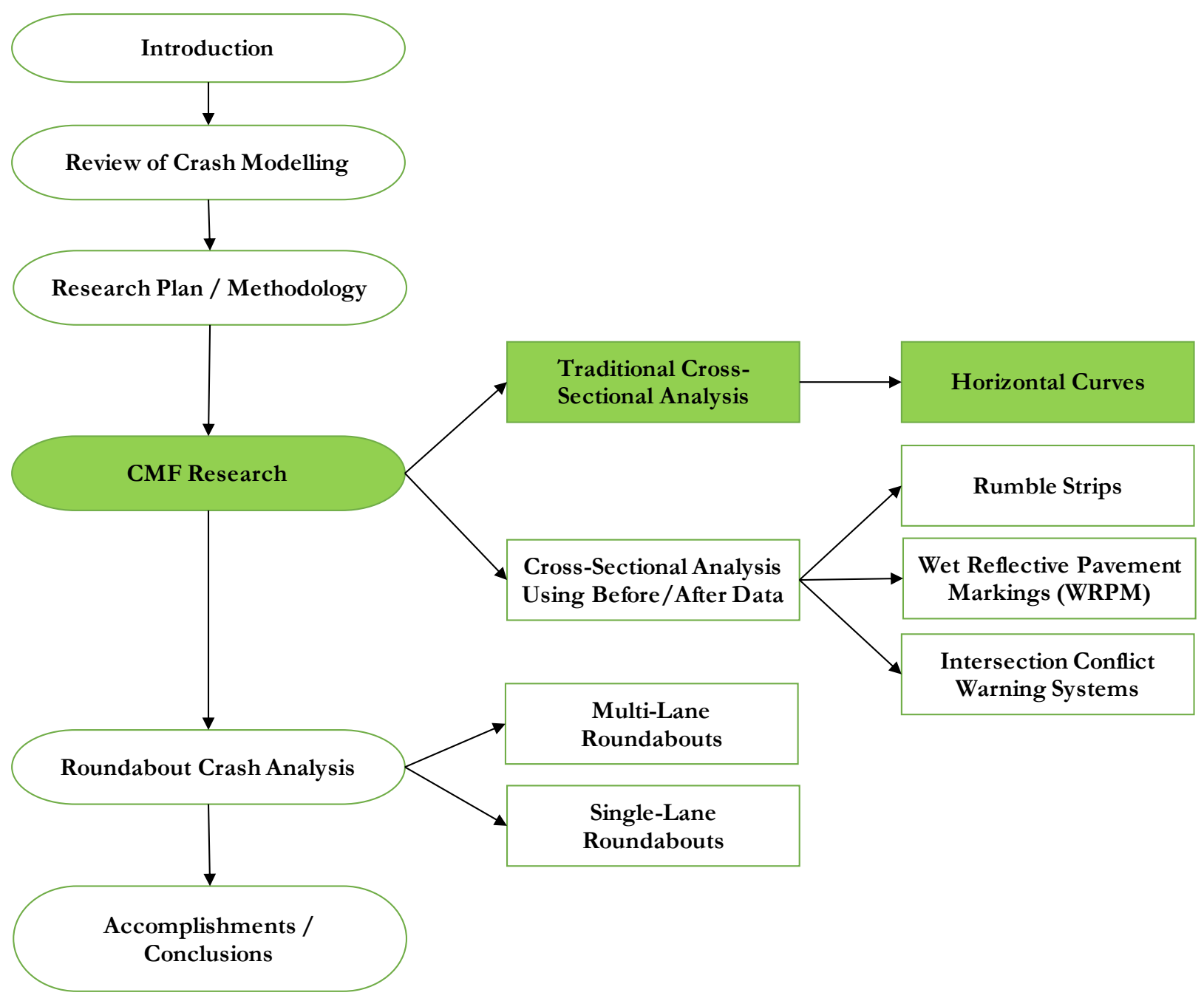

This chapter focuses on evaluating the potential of cross-sectional analysis for estimating CMFs in the absence of before-after data. Horizontal curves on rural two-lane highways are used as the design element in this evaluation. 
Evaluation using before-after data gives us the ability to accurately predict the change in crashes as they would have materialized in real life. Unfortunately, for some cases, collecting before-after data is not possible. An example of such a case would be changing the horizontal curvature of a road since there can literally be infinite combinations of curvature before and after a change. This chapter presents the first part of the CMF research component in this dissertation -- evaluating the potential of cross-sectional analysis for estimating CMFs in the absence of before-after data. The design element for this part is horizontal curvature on rural two-lane highways. The data used for this part came from the Washington State database in the Highway Safety Information System (HSIS, 2016). Crash prediction models are developed for (a) the curve sections incorporating the effects of radius, length, and shoulder width, and (b) the tangent sections up and down stream of the curve sections in question. Both sets of models account for gradient as a confounding variable. These models are then used as functions to derive the crash reduction rate (CRR) for contemplated changes in design, and subsequently the CMFs. The relationship between crashes at different volumes and deflection angles was also explored to get approximate estimates of CMFs for incremental increases in the radius, considering the differences in tangent curve lengths for two different radii.

\section{$\underline{4.1 \text { Background/Motivation }}$}

The design element of interest for this chapter is flattening of horizontal curves on rural 2-lane highways. The most well known and perhaps widely used model for predicting safety on rural 2-lane highways was developed by Zegeer et al. (1992). Zegeer et al. in their paper looked at the safety effects of various geometric improvements on horizontal curves. A study conducted by Hauer et al. (2000) concluded that the model developed by Zegeer et al. was the most useful 
and accurate model to account for safety on horizontal curves on rural 2-lane highways. Hauer et al. in their study expressed the Zegeer et al.'s model as a CMF, which was subsequently adopted by the Highway Safety Manual (AASTHO, 2010) to represent the effect of horizontal curvature changes. Equation 4-1 shows the Zegeer et al.'s model as it was adopted in the Highway Safety Manual (AASTHO, 2010).

$C M F_{\text {Horizontal Curves }}=\frac{1.55 L_{c}+\frac{80.2}{R}-0.012 S}{1.55 L_{c}}$

Where;

$L_{c}=$ Length of Curve (mi),

$R=$ Radius of Curvature (ft.), and

$S=$ Presence of Spiral Transitions: 1 if present, 0 if not present.

Many recent researchers have also tried to analyze safety on horizontal curves. Pitale et al. (2009) analyzed using in-vehicle technologies as means of reducing crashes along horizontal curves. Fitzpatrick et al. (2009) modeled horizontal curve safety with consideration for driveway density, whereas, Schneider et al. (2009) analyzed the effects of horizontal curves on truck safety.

Zegeer et al.'s model as adapted as a CMF by Highway Safety Manual (AASTHO, 2010) does not provide any interaction between horizontal curvature and the percent grade on rural 2-lane highways, and although the Highway Safety Manual (AASTHO, 2010) has a separate CMF representing the safety effects of percent grade (see Table 4.1), it does not provide any method 
to account for the interaction between curvature and grade. Thus, technically the horizontal curvature CMF presented in the Highway Safety Manual (AASTHO, 2010) assumes that horizontal curvature effects safety in the same way whether it is located on level roadway ( $0 \%$ grade), level grade $(\leq 3 \%)$, moderate grade (3-6\%), or steep grade $(\geq 6 \%)$. Furthermore, Zegeer et al.'s model assumes a linear relationship between crashes and volumes, as the non-linear relationship now commonly assumed was only suggested in later research.

Table 4.1 Highway Safety Manual CMFs for Grade of Roadway Segments

\begin{tabular}{|c|c|}
\hline Approximate Grade (\%) & $C M F$ \\
\hline \multirow{3}{*}{$\begin{array}{c}\text { Level Grade }(\leq 3 \%) \\
\text { Moderate Terrain }(3 \%<\text { Grade } \leq 6 \%) \\
\text { Steep Terrain }(>6 \%)\end{array}$} & 1.00 \\
\hline & 1.10 \\
\hline & 1.16 \\
\hline
\end{tabular}

Bauer \& Harwood (2013) in their study tried to address this issue by analyzing the safety affects for horizontal curve and grade combinations. They modeled horizontal curves on straight grades (both level and non-level alignments) with percent grades ranging from $0 \%$ to 9.67\% (see Equation 4-2). Furthermore, the CMF presented in the Highway Safety Manual does not consider differences in tangent lengths, although Zegeer et al. did develop a tangent model and suggested how it could be used when estimating the safety effect of changing horizontal curvature.

$C M F_{F \& I}=\exp \left[0.044 G+0.19 \ln \left(\frac{11460}{R}\right)+4.52\left(\frac{1}{R}\right)\left(\frac{1}{L_{c}}\right)\right]$

Where;

$L_{c}=$ Length of Curve (mi), 
$R=$ Radius of Curvature (ft.), and

$G=$ Percent Grade of Roadway.

The main objective of this chapter is to explore the development of crash modification functions (CMFunctions) to estimate CMFs in the scenario where different designs are explored to flatten an existing horizontal curve. This research builds upon previous research by Zegeer et al. (1992) and Bauer \& Harwood (2013). This research tries to improve the linear relationship between crashes and volume as shown by Zegeer et al. and focuses on curves on level grades $(\leq 3 \%)$, moderate grades $(3-6 \%)$, and steep grades $(>6 \%)$, so that the approach to developing CMFunctions for changing horizontal curvature as introduced by Bauer \& Harwood (2013) could be improved to be explored without the confounding effects of gradient.

For this purpose, crash prediction models were developed for (a) the curve sections incorporating the effects of radius, length, and shoulder width, and (b) the tangent sections up and down stream of the curve sections in question. The presentation of these models is followed by a discussion of how these models can be used as functions to derive the crash reduction rate (CRR) for contemplated changes in design and subsequently the CMFs. To further demonstrate the capabilities of the developed models, the relationship between crashes at different volumes and deflection angles were explored to get approximate estimates of CMFs for incremental increases in the radius, considering the differences in tangent curve lengths for two different radii. 


\subsection{Summary of Data}

The data for this chapter came from the Washington State database in the Highway Safety Information System (HSIS, 2016). The database consisted of over 11,200 km (7,000 mi) of data including roadway inventory, traffic volumes, crashes and curve/grade information. Some guidelines were set to select the sites that were to be used for the study. These guidelines were as follows;

- Roadway type should be rural 2-lane highways,

- Minimum curve radius should be $30.5 \mathrm{~m}$ (100 ft.),

- Maximum curve radius should be $3493 \mathrm{~m}$ (11460 ft.), and

- Posted Speed on the curve section should be between $50-60 \mathrm{mi} . / \mathrm{hr} \mathrm{(} 80-100$ $\mathrm{km} / \mathrm{hr})$.

The minimum curve radius of $100 \mathrm{ft}$. was selected per the guidelines in the Highway Safety Manual (AASTHO, 2010). Similarly, the maximum curve radius was used to $11,460 \mathrm{ft}$. as curves with radius higher than 11,460 ft. can be classified as tangents for all practical purposes (Bauer \& Harwood, 2013). The posted speed limits on the curved sections used were limited to between $\sim 80-100 \mathrm{~km} / \mathrm{hr}$ in order to avoid the bias in the data since sites with much lower speed limits will tend to have lower crashes.

Using these guidelines, a total of 3,088 curves were selected. Of the 3,088 curves selected; 2,024 were on level grades, 856 were on moderate grades, and 208 were on steep grades. The data for tangent sections up and down stream of the curves were also extracted from the 
database for 2,511 of the 3,088 curves. Tangent data cannot be extracted for all the 3,088 curves due to the very small lengths of some tangent sections and in some cases the inability to extract crash data for the particular section. Of the 2,511 tangents sections; 1,680 were on level grades, 694 were on moderate grades, and 137 were on steep grades.

Tables 4.2 and 4.3 show some summary statistics of these sites. The crash data for these sites were available for the seven-year period from 2005 - 2011. Crash statistics of the sites used in this study can be seen in Tables 4.4 and 4.5 .

Table 4.2 Summary Statistics for the Curved Segments

\begin{tabular}{|c|c|c|c|}
\hline \multicolumn{4}{|c|}{ Curved Segments on Level Grades ( $n=2024$ ) } \\
\hline Variable & Minimum & Maximum & Average \\
\hline$A A D T$ & 150.00 & 25204.00 & 2704.08 \\
\hline Radius (m) & 30.48 & 3493.01 & 624.82 \\
\hline Length $(m)$ & 18.25 & 1111.52 & 192.39 \\
\hline Shoulder (m) & 0.30 & 3.81 & 1.35 \\
\hline Grade (\%) & 0.00 & 3.00 & 0.89 \\
\hline \multicolumn{4}{|c|}{ Curved Segments on Moderate Grades $(n=856)$} \\
\hline Variable & Minimum & Maximum & Average \\
\hline$A A D T$ & 207.00 & 19831.00 & 2147.47 \\
\hline Radius (m) & 30.78 & 3493.01 & 507.56 \\
\hline Length $(m)$ & 19.81 & 914.57 & 186.64 \\
\hline Shoulder $(m)$ & 0.30 & 3.66 & 1.26 \\
\hline Grade (\%) & 3.01 & 6.00 & 4.76 \\
\hline \multicolumn{4}{|c|}{ Curved Segments on Steep Grades $(n=208)$} \\
\hline Variable & Minimum & Maximum & Average \\
\hline AADT & 208.00 & 8580.00 & 1476.61 \\
\hline Radius (m) & 31.09 & 3492.70 & 394.36 \\
\hline Length $(m)$ & 19.81 & 769.74 & 159.51 \\
\hline Shoulder (m) & 0.30 & 3.66 & 1.22 \\
\hline Grade (\%) & 6.01 & 9.47 & 6.51 \\
\hline
\end{tabular}

Note: $1 \mathrm{mi}=1609.34 \mathrm{~m}, 1 \mathrm{ft} .=0.3048 \mathrm{~m}$ 
Table 4.3 Summary Statistics for the Tangent Sections

\begin{tabular}{|c|c|c|c|}
\hline \multicolumn{4}{|c|}{ Tangents on Level Grades $(n=1680)$} \\
\hline Variable & Minimum & Maximum & Average \\
\hline AADT & 150.00 & 18745.00 & 2741.12 \\
\hline Length (m) & 161.22 & 7999.89 & 1717.63 \\
\hline Shoulder (m) & 0.30 & 3.81 & 1.38 \\
\hline Grade (\%) & 0.00 & 3.00 & 0.89 \\
\hline \multicolumn{4}{|c|}{ Tangents on Moderate Grades $(n=694)$} \\
\hline Variable & Minimum & Maximum & Average \\
\hline$A A D T$ & 207.00 & 18745.00 & 2204.31 \\
\hline Length $(m)$ & 160.94 & 7676.42 & 1343.89 \\
\hline Shoulder (m) & 0.30 & 3.66 & 1.31 \\
\hline Grade (\%) & 3.01 & 6.00 & 4.73 \\
\hline \multicolumn{4}{|c|}{ Tangents on Steep Grades $(n=137)$} \\
\hline Variable & Minimum & Maximum & Average \\
\hline$A A D T$ & 208.00 & 8580.00 & 1678.35 \\
\hline Length (m) & 162.64 & 7942.41 & 1063.94 \\
\hline Shoulder (m) & 0.30 & 3.66 & 1.18 \\
\hline Grade (\%) & 6.01 & 9.47 & 6.53 \\
\hline
\end{tabular}

Note: $1 \mathrm{mi}=1609.34 \mathrm{~m}, 1 \mathrm{ft} .=0.3048 \mathrm{~m}$

Table 4.4 Crash Statistics for the Curved Segments

\begin{tabular}{|c|c|c|c|}
\hline \multicolumn{4}{|c|}{ Curved Segments on Level Grades $(n=2024)$} \\
\hline Crash Totals (2005-2011) & Minimum & Maximum & Average \\
\hline \multirow{3}{*}{$\begin{array}{c}\text { Total } \\
\text { Fatal \& Injury } \\
\text { Property Damage Only }\end{array}$} & 0 & 24 & 1.1546 \\
\hline & 0 & 12 & 0.4619 \\
\hline & 0 & 15 & 0.6926 \\
\hline \multicolumn{4}{|c|}{ Curved Segments on Moderate Grades $(n=856)$} \\
\hline Crash Totals (2005-2011) & Minimum & Maximum & Average \\
\hline \multirow{3}{*}{$\begin{array}{c}\text { Total } \\
\text { Fatal \& Injury } \\
\text { Property Damage Only }\end{array}$} & 0 & 22 & 1.0338 \\
\hline & 0 & 12 & 0.4112 \\
\hline & 0 & 16 & 0.6226 \\
\hline \multicolumn{4}{|c|}{ Curved Segments on Steep Grades $(n=208)$} \\
\hline Crash Totals (2005-2011) & Minimum & Maximum & Average \\
\hline \multirow{3}{*}{$\begin{array}{c}\text { Total } \\
\text { Fatal \& Injury } \\
\text { Property Damage Only }\end{array}$} & 0 & 8 & 0.6298 \\
\hline & 0 & 5 & 0.2644 \\
\hline & 0 & 4 & 0.3653 \\
\hline
\end{tabular}


Table 4.5 Crash Statistics for the Tangent Sections

\begin{tabular}{|c|c|c|c|}
\hline \multicolumn{4}{|c|}{ Tangents on Level Grades $(n=1680)$} \\
\hline Crash Totals (2005-2011) & Minimum & Maximum & Average \\
\hline \multirow{3}{*}{$\begin{array}{c}\text { Total } \\
\text { Fatal \& Injury } \\
\text { Property Damage Only }\end{array}$} & 0 & 106 & 10.1997 \\
\hline & 0 & 47 & 3.9511 \\
\hline & 0 & 78 & 6.2485 \\
\hline \multicolumn{4}{|c|}{ Tangents on Moderate Grades $(n=694)$} \\
\hline Crash Totals (2005-2011) & Minimum & Maximum & Average \\
\hline \multirow{3}{*}{$\begin{array}{c}\text { Total } \\
\text { Fatal \& Injury } \\
\text { Property Damage Only }\end{array}$} & 0 & 80 & 7.0165 \\
\hline & 0 & 44 & 2.7391 \\
\hline & 0 & 53 & 4.2773 \\
\hline \multicolumn{4}{|c|}{ Tangents on Steep Grades $(n=137)$} \\
\hline Crash Totals (2005-2011) & Minimum & Maximum & Average \\
\hline \multirow{3}{*}{$\begin{array}{c}\text { Total } \\
\text { Fatal \& Injury } \\
\text { Property Damage Only }\end{array}$} & 0 & 60 & 5.4190 \\
\hline & 0 & 18 & 2.1820 \\
\hline & 0 & 43 & 3.2370 \\
\hline
\end{tabular}

\subsection{Model Fitting and Evaluation}

Consistent with state-of-the-art methods, generalized linear modelling, with the specification of a negative binomial (NB) error structure (as discussed in Section 3.4), was used to develop the crash prediction models (Persaud et al., 2010) using the SAS software (SAS, 2016).

\subsubsection{Model Fitting Using Curve Data}

Different models were developed for curves on level, moderate and steep grades. The model form used for developing the models for curved segments was as follows:

Crashes $/$ Year $=e^{\alpha} \times A A D T^{\beta_{1}} \times$ Radius $^{\beta_{2}} \times$ Length $^{\beta_{3}} \times e^{\beta_{4} \times \text { Shoulder Width }}$

Where;

$A A D T=$ Average annual daily traffic,

Radius $=$ Radius of curve in meters, 
Length $=$ Length of curve in meters, and

Shoulder Width $=$ Average of left and right side shoulder widths in meters.

Tables 4.6, 4.7 and 4.8 show the coefficient estimates, over dispersion parameters (K) and the goodness of prediction measures for models for various crash severities.

Table 4.6 Estimates \& Goodness of Fit Measures for the Curve Models on Level Grades

\begin{tabular}{|c|c|c|c|c|c|c|}
\hline \multicolumn{7}{|c|}{ Curved Segments on Level Grades } \\
\hline Crash Type & \multicolumn{2}{|c|}{ Total Crashes } & \multicolumn{2}{|c|}{ F\&I Crashes } & \multicolumn{2}{|c|}{ PDO Crashes } \\
\hline Coefficient & $\begin{array}{c}\text { Estimate } \\
\text { (Standard Error) }\end{array}$ & Pr>ChiSq & $\begin{array}{c}\text { Estimate } \\
\text { (Standard Error) }\end{array}$ & Pr>Chisq & $\begin{array}{c}\text { Estimate } \\
\text { (Standard Error) }\end{array}$ & Pr>ChiSq \\
\hline$\alpha$ & $-11.4015(0.3638)$ & $<0.0001$ & $-11.8006(0.4974)$ & $<0.0001$ & $-12.2855(0.4449)$ & $<0.0001$ \\
\hline 81 & $0.9176(0.0361)$ & $<0.0001$ & $0.9125(0.0498)$ & $<0.0001$ & $0.9185(0.0429)$ & $<0.0001$ \\
\hline 82 & $-0.4342(0.0383)$ & $<0.0001$ & $-0.5175(0.0549)$ & $<0.0001$ & $-0.3679(0.0457)$ & $<0.0001$ \\
\hline 83 & $0.9735(0.0482)$ & $<0.0001$ & $0.9783(0.0671)$ & $<0.0001$ & $0.9709(0.0571)$ & $<0.0001$ \\
\hline 84 & $-0.0434(0.0153)$ & 0.0372 & $-0.0442(0.0124)$ & 0.0183 & $-0.0582(0.0240)$ & 0.0810 \\
\hline$K$ & \multicolumn{2}{|l|}{0.4983} & \multicolumn{2}{|l|}{0.5531} & \multicolumn{2}{|l|}{0.5239} \\
\hline Crash Type & \multicolumn{2}{|c|}{ Total Crashes } & \multicolumn{2}{|c|}{ F\&I Crashes } & \multicolumn{2}{|c|}{ PDO Crashes } \\
\hline \multirow{3}{*}{$\begin{array}{c}\text { Avg. Obs. Crashes/Year/Site } \\
\text { MAD/Year/Site } \\
\text { MPE/Year/Site }\end{array}$} & \multicolumn{2}{|c|}{1.1546} & \multicolumn{2}{|c|}{0.4619} & \multicolumn{2}{|c|}{0.6926} \\
\hline & \multicolumn{2}{|l|}{0.1368} & \multicolumn{2}{|l|}{0.0736} & \multicolumn{2}{|l|}{0.0964} \\
\hline & \multicolumn{2}{|l|}{0.0140} & \multicolumn{2}{|l|}{0.0072} & \multicolumn{2}{|l|}{0.0096} \\
\hline
\end{tabular}

Table 4.7 Estimates \& Goodness of Fit Measures for the Curve Models on Moderate Grades

\begin{tabular}{|c|c|c|c|c|c|c|}
\hline \multicolumn{7}{|c|}{ Curved Segments on Moderate Grades } \\
\hline Crash Type & \multicolumn{2}{|c|}{ Total Crashes } & \multicolumn{2}{|c|}{ F\&I Crashes } & \multicolumn{2}{|c|}{ PDO Crashes } \\
\hline Coefficient & $\begin{array}{c}\text { Estimate } \\
\text { (Standard Error) }\end{array}$ & Pr>Chisq & $\begin{array}{c}\text { Estimate } \\
\text { (Standard Error) }\end{array}$ & Pr>ChiSq & $\begin{array}{c}\text { Estimate } \\
\text { (Standard Error) }\end{array}$ & Pr>ChiSq \\
\hline$\alpha$ & $-11.2799(0.5277)$ & $<0.0001$ & $11.0684(0.7468)$ & $<0.0001$ & $-12.5824(0.6466)$ & $<0.0001$ \\
\hline 81 & $0.9711(0.0548)$ & $<0.0001$ & $0.9027(0.0771)$ & $<0.0001$ & $0.9982(0.0653)$ & $<0.0001$ \\
\hline 82 & $-0.5951(0.0626)$ & $<0.0001$ & $-0.5330(0.0894)$ & $<0.0001$ & $-0.6344(0.0782)$ & $<0.0001$ \\
\hline 83 & $1.0593(0.0787)$ & $<0.0001$ & $0.9163(0.1107)$ & $<0.0001$ & $1.1595(0.0962)$ & $<0.0001$ \\
\hline 84 & $-0.1032(0.0493)$ & 0.0930 & $-0.2211(0.0372)$ & 0.0593 & $-0.0405(0.0232)$ & 0.1639 \\
\hline$K$ & \multicolumn{2}{|l|}{0.3691} & \multicolumn{2}{|l|}{0.5703} & \multicolumn{2}{|l|}{0.3480} \\
\hline Crash Type & \multicolumn{2}{|c|}{ Total Crashes } & \multicolumn{2}{|c|}{ F\&I Crashes } & \multicolumn{2}{|c|}{ PDO Crashes } \\
\hline \multirow{3}{*}{$\begin{array}{c}\text { Avg. Obs. Crashes/Year/Site } \\
\text { MAD/Year/Site } \\
\text { MPE/Year/Site }\end{array}$} & \multicolumn{2}{|c|}{1.0338} & \multicolumn{2}{|c|}{0.4112} & \multicolumn{2}{|c|}{0.6226} \\
\hline & \multicolumn{2}{|l|}{0.1185} & \multicolumn{2}{|c|}{0.0668} & \multicolumn{2}{|l|}{0.0818} \\
\hline & \multicolumn{2}{|l|}{0.0181} & \multicolumn{2}{|c|}{0.0099} & \multicolumn{2}{|l|}{0.0136} \\
\hline
\end{tabular}


Table 4.8 Estimates \& Goodness of Fit Measures for the Curve Models on Steep Grades

\begin{tabular}{|c|c|c|c|c|c|c|}
\hline \multicolumn{7}{|c|}{ Curved Segments on Steep Grades } \\
\hline \multirow{2}{*}{$\begin{array}{l}\text { Crash Type } \\
\text { Coefficient }\end{array}$} & \multicolumn{2}{|c|}{ Total Crashes } & \multicolumn{2}{|c|}{ F\&I Crashes } & \multicolumn{2}{|c|}{ PDO Crashes } \\
\hline & $\begin{array}{c}\text { Estimate } \\
\text { (Standard Error) }\end{array}$ & $\operatorname{Pr}>$ ChiSq & $\begin{array}{c}\text { Estimate } \\
\text { (Standard Error) }\end{array}$ & Pr>ChiSq & $\begin{array}{c}\text { Estimate } \\
\text { (Standard Error) }\end{array}$ & Pr>ChiSq \\
\hline$\alpha$ & $-10.1187(1.2819)$ & $<0.0001$ & $-10.9298(2.0029)$ & $<0.0001$ & $-10.9124(1.2739)$ & $<0.0001$ \\
\hline 81 & $0.9242(0.1456)$ & $<0.0001$ & $0.9498(0.2284)$ & $<0.0001$ & $0.8869(0.1363)$ & $<0.0001$ \\
\hline 82 & $-0.8275(0.1703)$ & $<0.0001$ & $-1.0616(0.2703)$ & $<0.0001$ & $-0.6589(0.1862)$ & 0.0004 \\
\hline 83 & $1.1169(0.2013)$ & $<0.0001$ & $1.3328(0.3176)$ & $<0.0001$ & $1.0063(0.2009)$ & $<0.0001$ \\
\hline 84 & $-0.0302(0.0128)$ & 0.1783 & $-0.0992(0.0679)$ & 0.1112 & $-0.0916(0.0553)$ & 0.1953 \\
\hline$K$ & \multicolumn{2}{|l|}{0.5917} & \multicolumn{2}{|l|}{1.1166} & \multicolumn{2}{|l|}{0.2283} \\
\hline Crash Type & \multicolumn{2}{|c|}{ Total Crashes } & \multicolumn{2}{|c|}{ F\&I Crashes } & \multicolumn{2}{|c|}{ PDO Crashes } \\
\hline Avg. Obs. Crashes/Year/Site & \multicolumn{2}{|c|}{0.6298} & \multicolumn{2}{|c|}{0.2644} & \multicolumn{2}{|c|}{0.3635} \\
\hline MAD/Year/Site & \multicolumn{2}{|l|}{0.0875} & \multicolumn{2}{|l|}{0.0491} & \multicolumn{2}{|l|}{0.0556} \\
\hline MPE/Year/Site & \multicolumn{2}{|l|}{0.0269} & \multicolumn{2}{|l|}{0.0174} & \multicolumn{2}{|l|}{0.0160} \\
\hline
\end{tabular}

As can be seen from Tables $4.6,4.7$ and 4.8 , the estimates of $\beta_{1}, \beta_{2}, \beta_{3}$ and $\beta_{4}$ have intuitive signs and are all highly significant $(\mathrm{P}<0.05)$ for almost all of the models. The goodness of prediction measures also suggests reasonably good fits in that the MAD/year/site (Mean Absolute deviation) and the MPE/year/site (Mean Prediction Error) for all the models are small (close to zero in all cases) when compared to the average observed crashes per year per site. For example, the MAD/year/site for total crashes on curves on level grades is 0.137 compared to an average of $\sim 1.15$ crashes per year/site. Figures $4.1,4.2$ and 4.3 shows the CURE plots for all crash severities. 

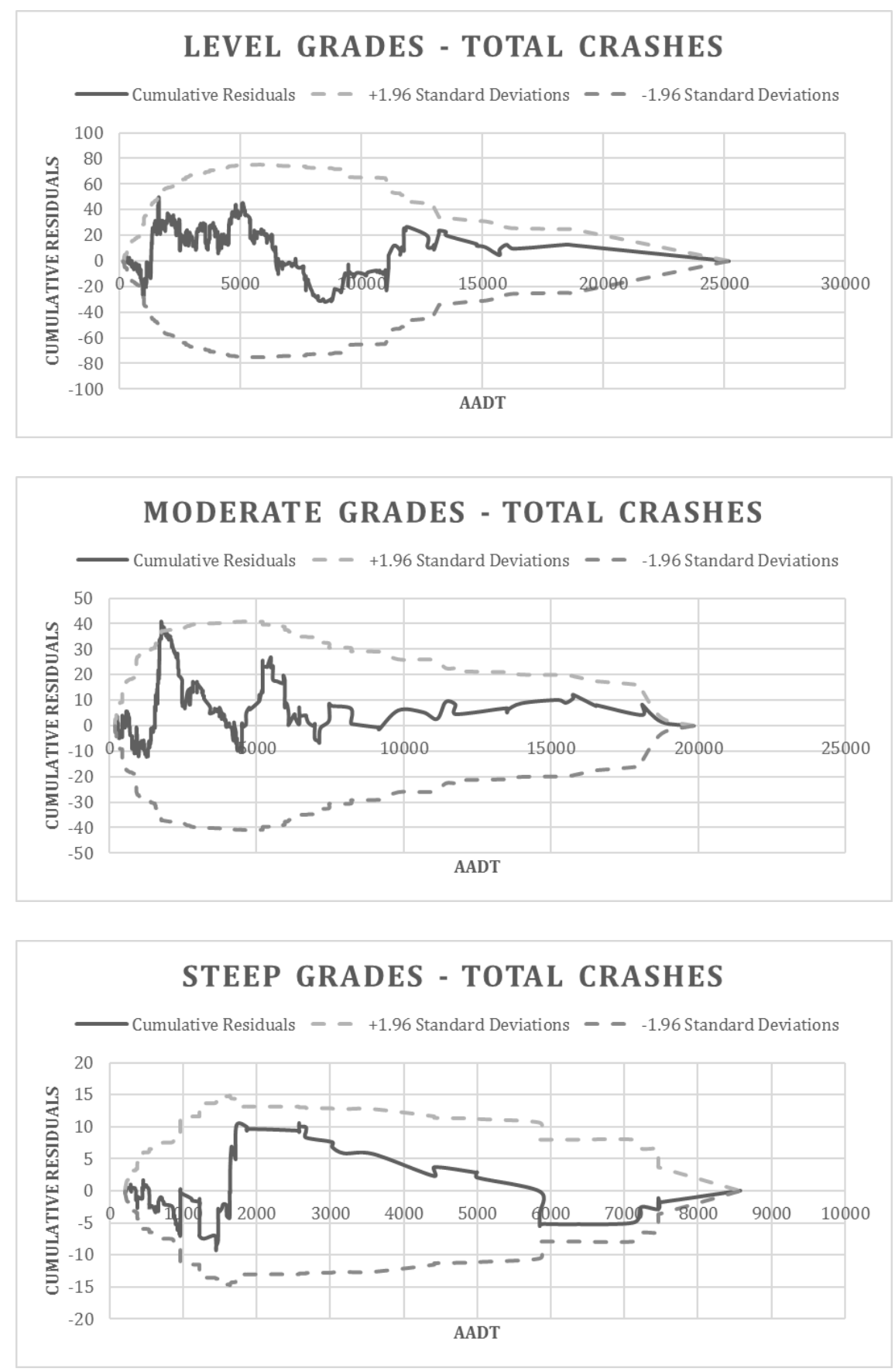

Figure 4.1 CURE Plots for Total Crashes (Curved Segments) 

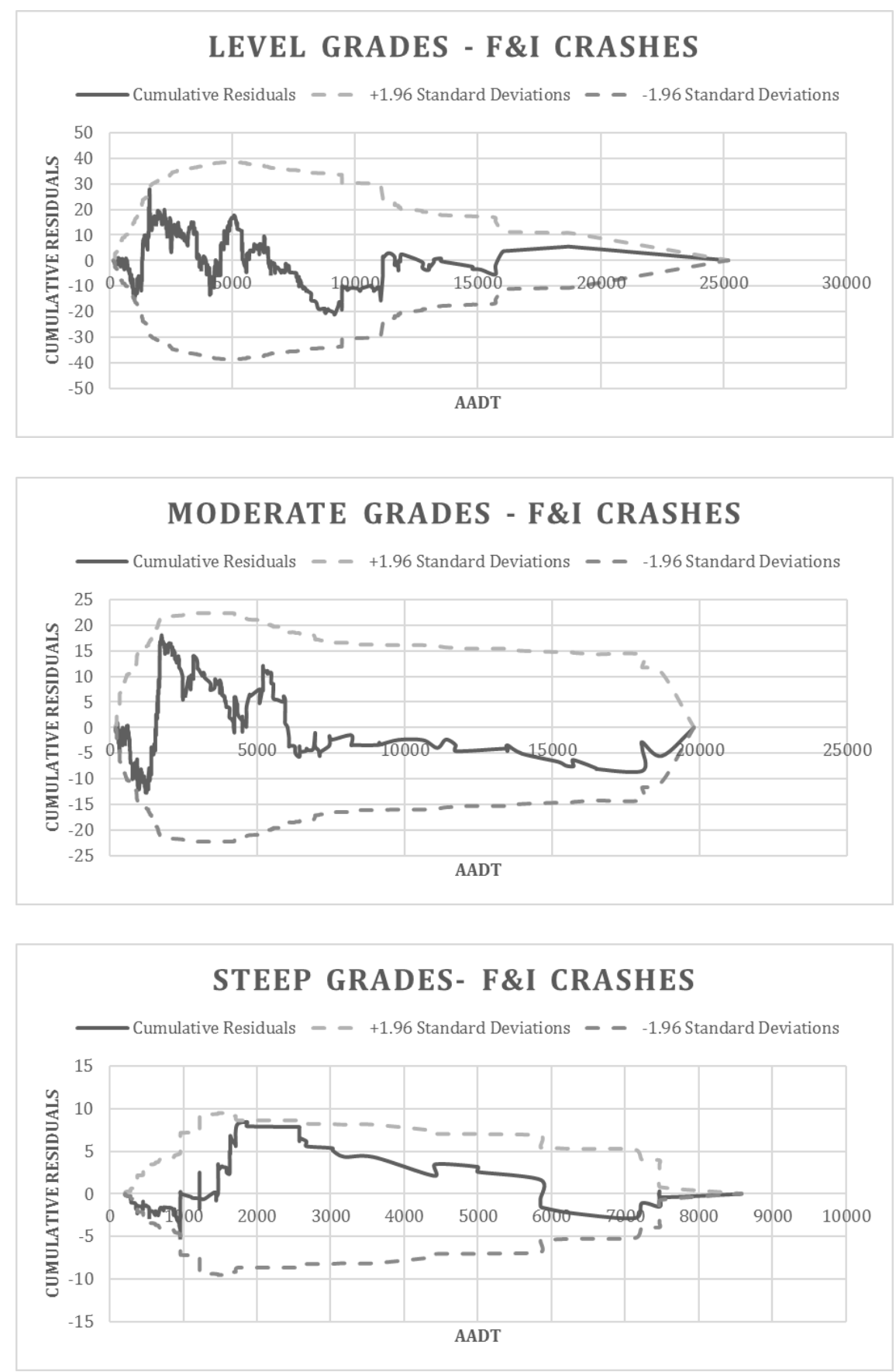

Figure 4.2 CURE Plots for F\&I Crashes (Curved Segments) 

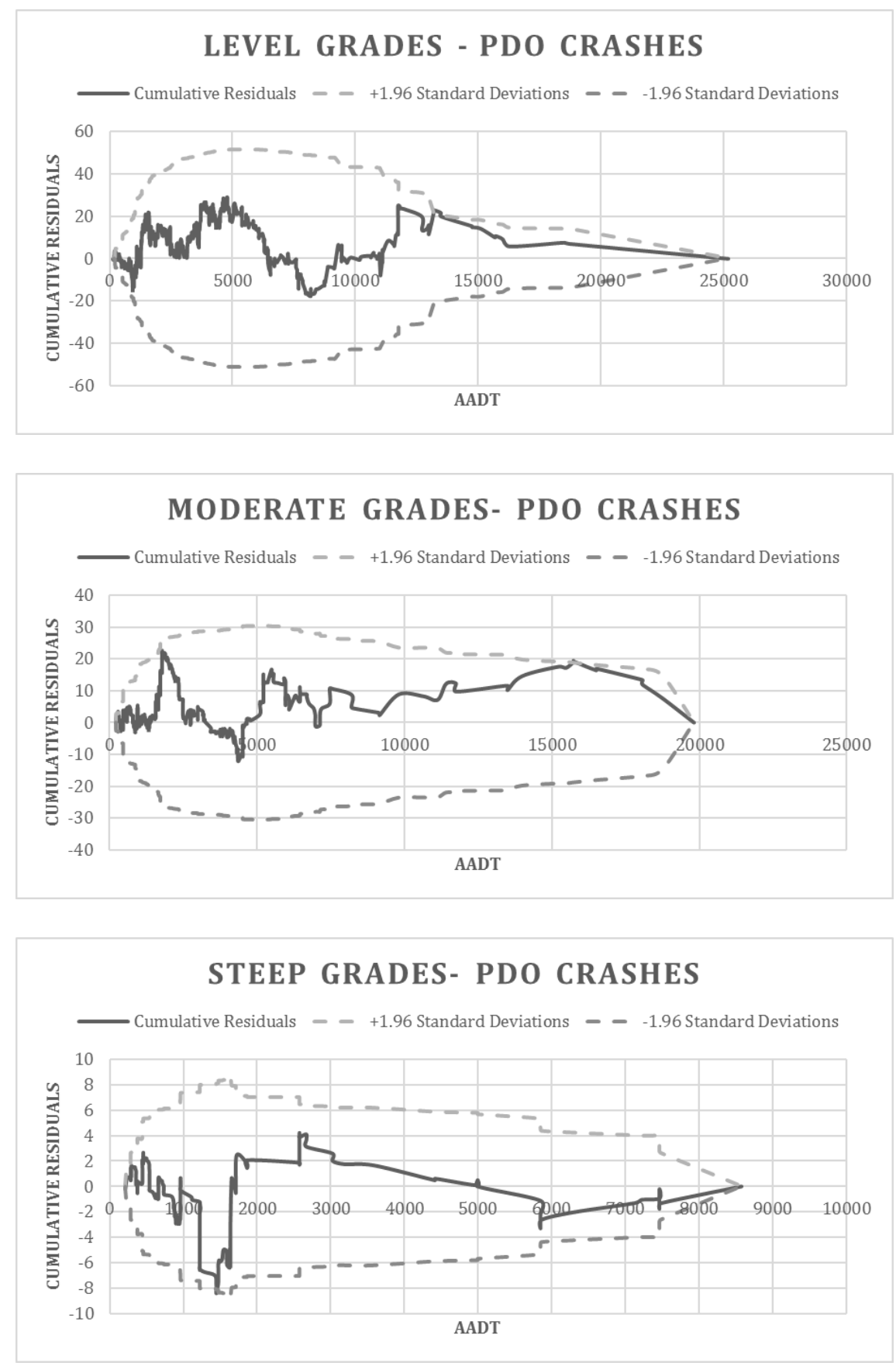

Figure 4.3 CURE Plots for PDO Crashes (Curved Segments) 
The CURE plots for total crashes on curved segments can be seen in Figure 4.1. The CURE plot for total crashes on curved segments on level grades show that the cumulative residuals lie within the $95 \%$ confidence boundaries and oscillate consistently showing little or no bias. The plot for total crashes on curved segments on moderate grades also oscillate well but does show a long vertical jump which takes the plot slightly outside the $95 \%$ confidence boundary. This may well suggest of a possible outlier in the data that makes the plot behave like this (Hauer, 2014). Apart from this, it can be said that the CURE plot does oscillate consistently showing little or no bias. The plot for total crashes on curved segments on steep grades does show a steep vertical run as well, which may again be indicative of an outlier (Hauer, 2014), but as can be seen the plot stays consistently well within the $95 \%$ confidence boundaries and oscillates consistently suggesting of a good overall fit.

The CURE plots for fatal and injury (F\&I) crashes on curved segments can be seen in Figure 4.2. It can be seen that for F\&I crashes on curved segments on level and steep grades the cumulative residuals oscillate consistently and lie well within the $95 \%$ confidence intervals suggesting of a good fit. The plot for F\&I crashes on curved segments on moderate grades also oscillate consistently and stays well within the $95 \%$ confidence intervals, but it does show a steep vertical jump indicating of a possible outlier (Hauer, 2014). Overall, based on consistent oscillation and staying well within the confidence boundaries it can be said that the CURE plot do suggest of a good overall fit.

The CURE plots for PDO crashes on curved segments can be seen in Figure 4.3. It can be seen that for PDO crashes on curved segments on level and moderate the CURE plot oscillates consistently and stays well within the $95 \%$ confidence intervals suggesting a good fit. The plot 
for PDO crashes on curved segments on steep grades though shows that the plot does stay well within the $95 \%$ confidence boundaries, it also a steep vertical jump and a drop suggesting of two possible outliers in the data (Hauer, 2014). Furthermore, the plot also shows a section of decreasing run which is indicative of under-prediction by the model in that range (Hauer, 2010). Overall, since the plot stays within the $95 \%$ boundaries, it can be said that the model does provide a good overall fit but does under predict the crashes on a small range of data.

Overall, based on all the goodness of prediction measures characterized by very low MAD/year/site and MPE/year/site values and good CURE plots in almost all the cases it can be said that the models developed are well fit and can predict crashes reasonably close to reality.

\subsubsection{Model Fitting Using Up and Downstream Tangent Data}

Different models were developed for tangents on level, moderate and steep grades. The model form used for developing the models for tangent sections was as follows:

Crashes $/$ Year $=e^{\alpha} \times A A D T^{\beta_{1}} \times$ Length $^{\beta_{2}}$

Where;

$A A D T=$ Average annual daily traffic, and

Length $=$ Length of tangent section in meters.

Tables 4.9, 4.10 and 4.11 show the coefficient estimates, over dispersion parameters $(\mathrm{K})$ and the goodness of prediction measures for models distinguished by their specific crash types. 
Note that shoulder width is not included in this models because it had a small and insignificant effect. CURE plots for all the models are shown in Figures 4.4, 4.5 and 4.6.

Table 4.9 Estimates \& Goodness of Fit Measures for the Tangents on Level Grades

\begin{tabular}{|c|c|c|c|c|c|c|}
\hline \multicolumn{7}{|c|}{ Tangents on Level Grades } \\
\hline Crash Type & \multicolumn{2}{|c|}{ Total Crashes } & \multicolumn{2}{|c|}{ F\&I Crashes } & \multicolumn{2}{|c|}{ PDO Crashes } \\
\hline Coefficient & $\begin{array}{c}\text { Estimate } \\
\text { (Standard Error) }\end{array}$ & Pr>Chisq & $\begin{array}{c}\text { Estimate } \\
\text { (Standard Error) }\end{array}$ & Pr>Chisq & $\begin{array}{c}\text { Estimate } \\
\text { (Standard Error) }\end{array}$ & Pr>Chisq \\
\hline$\alpha$ & $-14.3378(0.2052)$ & $<0.0001$ & $-14.9101(0.2323)$ & $<0.0001$ & $-15.0735(0.2356)$ & $<0.0001$ \\
\hline 81 & $0.9380(0.0190)$ & $<0.0001$ & $0.9224(0.0229)$ & $<0.0001$ & $0.9455(0.0215)$ & $<0.0001$ \\
\hline 82 & $0.9727(0.0177)$ & $<0.0001$ & $0.9407(0.0218)$ & $<0.0001$ & $0.9965(0.0202)$ & $<0.0001$ \\
\hline$K$ & \multicolumn{2}{|l|}{0.2089} & \multicolumn{2}{|l|}{0.1812} & \multicolumn{2}{|l|}{0.2081} \\
\hline Crash Type & \multicolumn{2}{|c|}{ Total Crashes } & \multicolumn{2}{|c|}{ F\&I Crashes } & \multicolumn{2}{|c|}{ PDO Crashes } \\
\hline \multirow{3}{*}{$\begin{array}{c}\text { Avg. Obs. Crashes/Year/Site } \\
\text { MAD/Year/Site } \\
\text { MPE/Year/Site }\end{array}$} & \multicolumn{2}{|c|}{10.1997} & \multicolumn{2}{|c|}{3.9511} & \multicolumn{2}{|c|}{6.2485} \\
\hline & \multicolumn{2}{|l|}{0.5942} & \multicolumn{2}{|l|}{0.2648} & \multicolumn{2}{|l|}{0.3987} \\
\hline & \multicolumn{2}{|l|}{0.0896} & \multicolumn{2}{|l|}{0.0366} & \multicolumn{2}{|l|}{0.0586} \\
\hline
\end{tabular}

Table 4.10 Estimates \& Goodness of Fit Measures for the Tangents on Moderate Grades

\begin{tabular}{|c|c|c|c|c|c|c|}
\hline \multicolumn{7}{|c|}{$\begin{array}{l}\text { Tangents on Moderate Grades } \\
\text { Total Crashes F\&I Crashes }\end{array}$} \\
\hline Coefficient & $\begin{array}{c}\text { Estimate } \\
\text { (Standard Error) }\end{array}$ & Pr>ChiSq & $\begin{array}{c}\text { Estimate } \\
\text { (Standard Error) }\end{array}$ & Pr>ChiSq & $\begin{array}{c}\text { Estimate } \\
\text { (Standard Error) }\end{array}$ & Pr>ChiSq \\
\hline$\alpha$ & $-13.6862(0.3175)$ & $<0.0001$ & $-14.2403(0.3869)$ & $<0.0001$ & $-14.5069(0.3491)$ & $<0.0001$ \\
\hline 81 & $0.9365(0.0304)$ & $<0.0001$ & $0.9123(0.0359)$ & $<0.0001$ & $0.9714(0.0327)$ & $<0.0001$ \\
\hline 82 & $0.8942(0.0290)$ & $<0.0001$ & $0.8701(0.0352)$ & $<0.0001$ & $0.8996(0.0315)$ & $<0.0001$ \\
\hline$K$ & \multicolumn{2}{|l|}{0.2060} & \multicolumn{2}{|l|}{0.1637} & \multicolumn{2}{|l|}{0.1648} \\
\hline Crash Type & \multicolumn{2}{|c|}{ Total Crashes } & \multicolumn{2}{|c|}{ F\&I Crashes } & \multicolumn{2}{|c|}{ PDO Crashes } \\
\hline Avg. Obs. Crashes/Year/Site & \multicolumn{2}{|l|}{7.0165} & \multicolumn{2}{|l|}{2.7391} & \multicolumn{2}{|l|}{4.2773} \\
\hline MAD/Year/Site & \multicolumn{2}{|l|}{0.4364} & \multicolumn{2}{|l|}{0.1981} & \multicolumn{2}{|l|}{0.2848} \\
\hline MPE/Year/Site & \multicolumn{2}{|l|}{0.1097} & \multicolumn{2}{|l|}{0.0433} & \multicolumn{2}{|l|}{0.0725} \\
\hline
\end{tabular}


Table 4.11 Estimates \& Goodness of Fit Measures for the Tangents on Steep Grades

Tangents on Steep Grades

\begin{tabular}{|c|c|c|c|c|c|c|}
\hline \multirow{2}{*}{$\begin{array}{l}\text { Crash Type } \\
\text { Coefficient }\end{array}$} & \multicolumn{2}{|c|}{ Total Crashes } & \multicolumn{2}{|c|}{ F\&I Crashes } & \multicolumn{2}{|c|}{ PDO Crashes } \\
\hline & $\begin{array}{c}\text { Estimate } \\
\text { (Standard Error) }\end{array}$ & Pr>ChiSq & $\begin{array}{c}\text { Estimate } \\
\text { (Standard Error) }\end{array}$ & Pr>ChiSq & $\begin{array}{c}\text { Estimate } \\
\text { (Standard Error) }\end{array}$ & Pr>Chisq \\
\hline$\alpha$ & $-12.3217(0.7672)$ & $<0.0001$ & $-13.0329(0.9491)$ & $<0.0001$ & $-13.5587(0.8428)$ & $<0.0001$ \\
\hline 81 & $0.8733(0.0905)$ & $<0.0001$ & $0.8466(0.1061)$ & $<0.0001$ & $0.9257(0.0963)$ & $<0.0001$ \\
\hline 82 & $0.7737(0.0894)$ & $<0.0001$ & $0.7835(0.1076)$ & $<0.0001$ & $0.8132(0.0970)$ & $<0.0001$ \\
\hline$K$ & \multicolumn{2}{|c|}{0.3709} & \multicolumn{2}{|l|}{0.3557} & \multicolumn{2}{|l|}{0.2866} \\
\hline Crash Type & \multicolumn{2}{|c|}{ Total Crashes } & \multicolumn{2}{|c|}{ F\&I Crashes } & \multicolumn{2}{|c|}{ PDO Crashes } \\
\hline Avg. Obs. Crashes/Year/Site & \multicolumn{2}{|c|}{5.419} & \multicolumn{2}{|c|}{2.1820} & \multicolumn{2}{|c|}{3.237} \\
\hline MAD/Year/Site & \multicolumn{2}{|l|}{0.4206} & \multicolumn{2}{|l|}{0.1999} & \multicolumn{2}{|l|}{0.2610} \\
\hline MPE/Year/Site & \multicolumn{2}{|l|}{0.3127} & \multicolumn{2}{|l|}{0.1127} & \multicolumn{2}{|l|}{0.1962} \\
\hline
\end{tabular}

As can be seen from Tables $4.9,4.10$ and 4.11 , the estimates of $\beta_{1}$ and $\beta_{2}$ are highly significant $(\mathrm{P}<0.01)$ for all the models. The goodness of prediction measures also suggests reasonably good fits in that the MAD/year/site (Mean Absolute Deviation) and the MPE/year/site (Mean Prediction Error) for all the models are small when compared to the average observed crashes per year per site (close to zero in all cases). For example, for tangents on level grades, the MAD/year/site is 0.594 and MPE/year/site is 0.090 compared to an average of $\sim 10.19$ total crashes per year/site. Similarly, for tangents on steep grades, the MAD/year/site is 0.420 and MPE/year/site is 0.312 compared to an average of $\sim 5.42$ total crashes per year/site. 

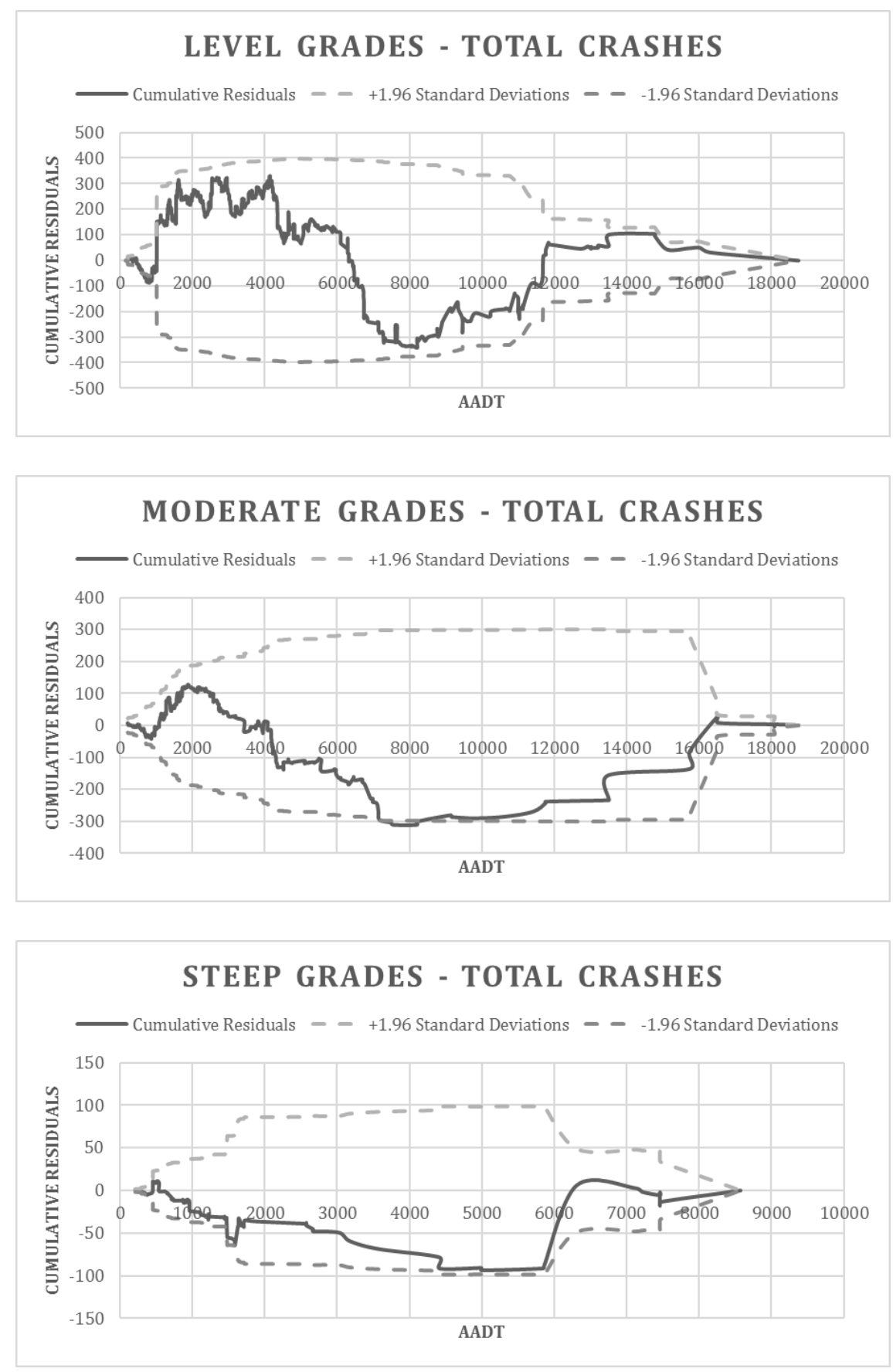

Figure 4.4 CURE Plots for Total Crashes (Tangent Sections) 

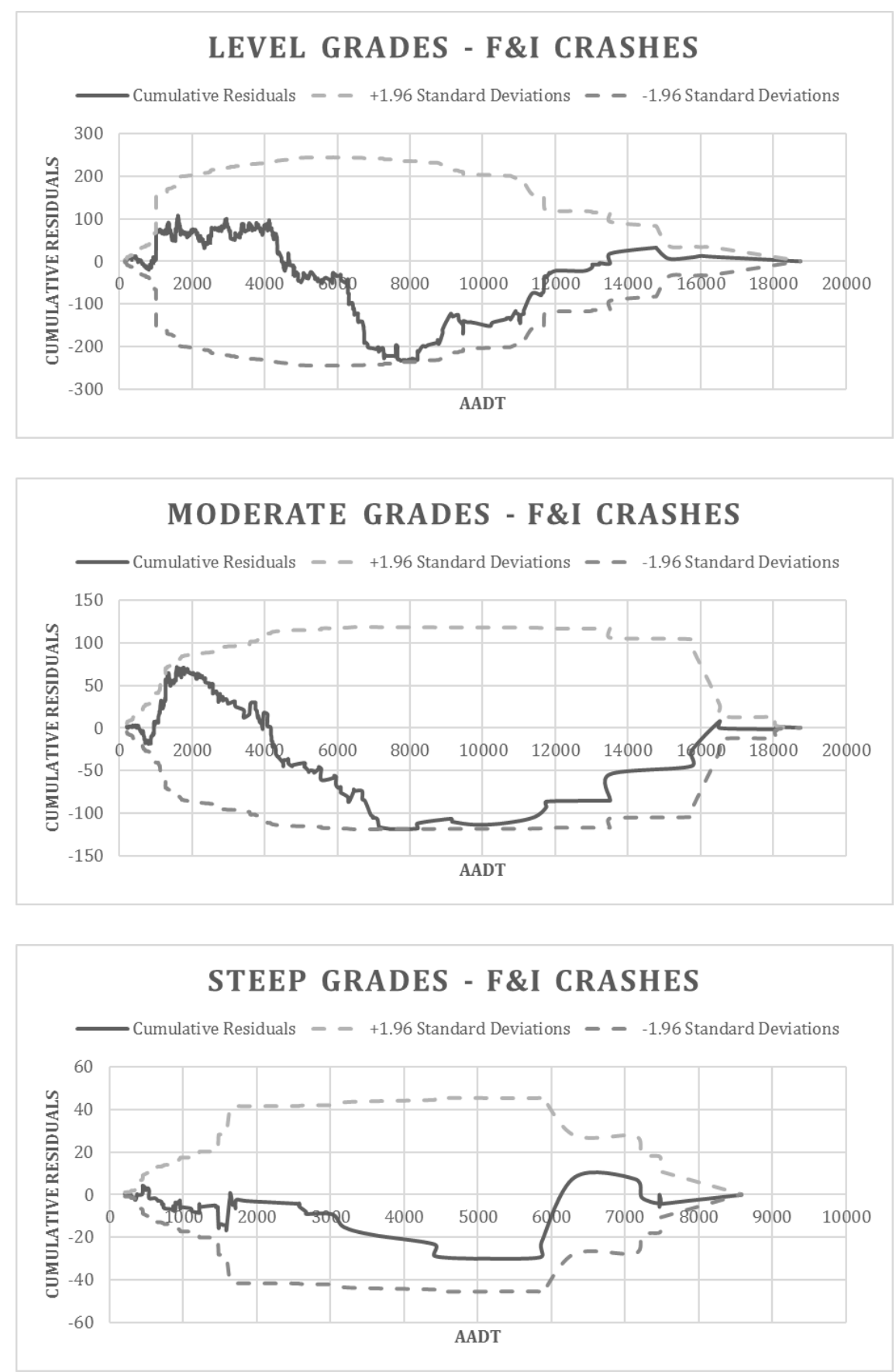

Figure 4.5 CURE Plots for F\&I Crashes (Tangent Sections) 

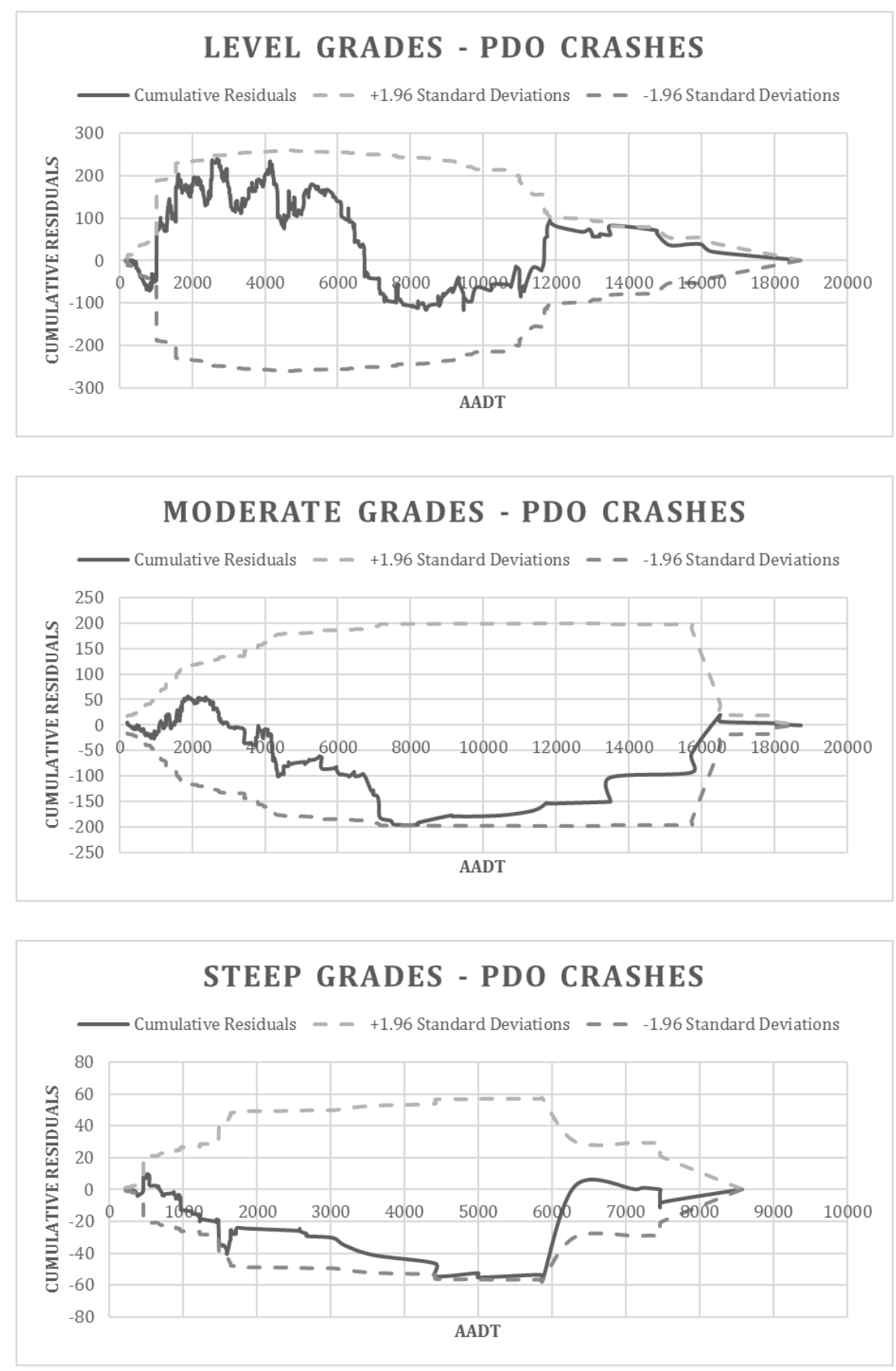

Figure 4.6 CURE Plots for PDO Crashes (Tangent Sections) 
The CURE plots for total crashes on tangent sections can be seen in Figure 4.4. The CURE plot for total crashes on tangent sections on level grades show that the cumulative residuals lie well within the $95 \%$ confidence boundaries and oscillate consistently showing little or no bias. The plot for total crashes on tangent sections on moderate grades also oscillate well but does show a long section of continuously increasing run suggesting of under/over prediction in this range (Hauer, 2014). Apart from this, it can be said that the CURE plot does oscillate consistently showing little or no bias. The plot for total crashes on tangent sections on steep grades does show a long sections of decreasing run, which may again be indicative of over/under prediction by the model in this range (Hauer, 2014), but as can be seen the plot stays consistently well with the $95 \%$ confidence boundaries suggesting of a good overall fit.

The CURE plots for fatal and injury (F\&I) crashes on tangent sections can be seen in Figure 4.5. It can be seen that for F\&I crashes on tangent sections on level grades the cumulative residuals oscillate consistently and lie well within the $95 \%$ confidence intervals, suggesting a good fit. The plots for F\&I crashes on tangent sections on moderate and steep grades also oscillate consistently and stay well within the $95 \%$ confidence intervals, but they both show long increasing and decreasing runs, respectively, which may be indicative of over/under prediction by the models (Hauer, 2014). Overall, based on consistent oscillation and staying well within the confidence boundaries it can be said that these CURE plots do suggest of a good overall fit.

The CURE plots for PDO crashes on tangent sections can be seen in Figure 4.6. It can be seen that the CURE plot for PDO crashes on tangent sections on level grades do oscillate consistently and stay well within the $95 \%$ confidence intervals suggesting of a good fit. Similar 
to F\&I crashes, the CURE plots for PDO crashes on tangent sections on moderate and steep grades stay well within the $95 \%$ confidence intervals, but they both show long increasing and decreasing runs, respectively, which may be indicative of over/under prediction by the models (Hauer, 2014). Overall, based on consistent oscillation and staying well within the confidence boundaries it can be said that these CURE plots do suggest of a good overall fit.

Overall, based on all the goodness of prediction measures characterized by very low MAD/year/site and MPE/year/site values and good CURE plots in almost all the cases it can be said that the models developed are well fit and can predict crashes reasonably close to reality.

\subsection{Application of Crash Prediction Models for Estimating Crash Modification Factors for Curve Flattening}

In order to assess the safety impacts of flattening a curve, it is essential to analyse the study area that extends beyond the limits of the smaller radii curve. The reason behind this is that when a curve is flattened (i.e. going from small radii to a larger radii), tangent sections from either ends are taken away to pave way for the larger radii curve. This phenomenon is further illustrated in Figure 4.7 below, as used by Hauer (1999) in his paper.

Thus, to accurately compare crashes between the old curve and the flattened curve, one should also account for crashes on the tangent section that was removed to accommodate the longer length of the flattened curve. Curved segments with spirals were considered in calculating tangent lengths for the entire curved segment, and the spiral lengths were added to the curve length to apply the models to Equation 4-3. 


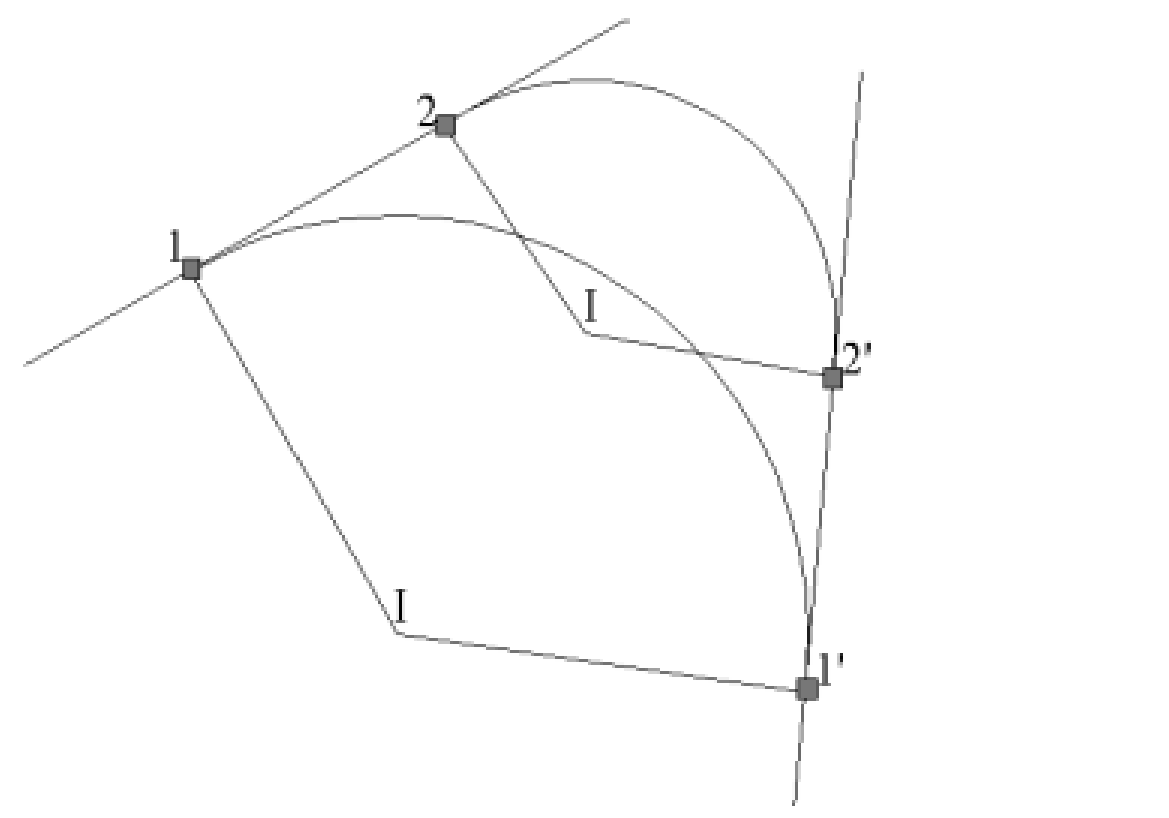

Figure 4.7 Illustration of Curve Flattening (going from 1-2-2'-1' to 1-I-1') for a given Deflection Angle (Hauer, 1999)

\subsubsection{Crash Reduction Rate (CRR) \& Crash Modification Factor (CMF) Estimation Methodology}

The crash reduction rate could be simply found by using the following equation;

$C R R=\frac{\text { Crashes }_{\text {old section }}-\text { Crashes }_{\text {new section }}}{\text { Crashes }_{\text {old section }}}$

When going from a small radii curve to a larger radii curve the crashes on the old section consist of crashes on the old curved segment and the crashes on the old tangent segments. The crashes on the new section would simply be the crashes on the flattened curve section with the larger radii. The curved segment to which the model is applied would include the circular curve plus the spirals, where these are used. Using the models shown in Equations 4-3 and 4-4, we can derive the total crashes on curved segments and tangent sections on level grades using the 
following equations (similar equations for moderate and steep grades can be derived using information from Tables 4.6-4.11):

$C_{\text {Curved Segments }}=$

$e^{-11.4015} \times A A D T^{0.9176} \times$ Radius $^{-0.4342} \times$ Length $^{0.9735} \times e^{-0.0434 \times \text { Shoulder Width }}$

$C_{\text {Tangent }}=e^{-14.3378} \times A A D T^{0.9380} \times$ Length $^{0.9727}$

Using Equations 4-5, 4-6 and 4-7 we can derive the crash modification function (CMFunction) that could be used to assess different scenarios of curve flattening and get a crash modification factor $(\mathrm{CMF})$ for them.

CMFunction $=1-\frac{\left(C_{\text {old curved segment }}+C_{\text {old tangent }}\right)-C_{\text {new } \text { curved segment }}}{\left(C_{\text {old curved segement }}+C_{\text {old tangent }}\right)}$

\subsubsection{CMF Estimates for Incrementally Increasing Radius over the Minimum Value Required at Certain Design Speeds}

The Transportation Association of Canada's Geometric Design Guide for Canadian Roads (TAC, 1999) specifies minimum radius requirements for different design speeds at different super elevation rates. For this study, the maximum super elevation rate of $0.06 \mathrm{~m} / \mathrm{m}$ was used because, according to TAC's Geometric Design Guide, in rural areas, the maximum super elevation rate of $0.06 \mathrm{~m} / \mathrm{m}$ is gaining more acceptance as it results in better horizontal alignment consistent with driver expectations for cases where minimum radii are used.

In this section, minimum radius for design speeds of 80,90 , and $100 \mathrm{~km} / \mathrm{h}$ were used as the base case to derive CMFs for increasing the radius by a factor of 1.10, 1.25, 1.50 and 2.00 over 
the minimum radius at different $\mathrm{AADTs}\left(15^{\text {th }}, 50^{\text {th }}\right.$, and the $85^{\text {th }}$ percentile of the dataset $)$ and deflection angles. Spiral lengths specified in the TAC's Geometric Design Guide for the appropriate radii were used. The results of this analysis for segments on level, moderate and steep grades (along with the standard errors) are shown in Tables 4.12, 4.13 and 4.14 respectively. The standard errors for the CMF estimates were calculated using the methodology explained by Bahar et al. (2007). The essence of the methodology is as follows;

1. Estimate the CMF from the regression model using the value of the coefficient(s) of the variable(s) of interest equal to the point estimate plus one standard deviation,

2. Estimate the CMF from the regression model using the value of the coefficient(s) of the variable(s) of interest equal to the point estimate minus one standard deviation,

3. Estimate the standard error of the CMF as one half of the absolute value of the difference between estimates from steps 1 and 2 .

For segments on level grades, it can be seen from Table 4.12 that the CMF estimates for a certain design speed and deflection angle follow a similar trend irrespective of the AADT. For example, at a design speed of $80 \mathrm{~km} / \mathrm{h}$, the $\mathrm{CMF}$ for increasing the radius by a factor 1.50 at deflection angle of $90^{\circ}$ is $\sim 0.880$ irrespective of the AADT. Similarly, at a design speed of 100 $\mathrm{km} / \mathrm{h}$, the CMF for increasing the radius by a factor of 2 at deflection angle of $30^{\circ}$ is $\sim 0.720$, irrespective of the AADT. It can also be seen that even at different speeds, the CMF estimates for a certain scenario seem to be following a similar trend. 
Table 4.12 Total Crashes CMF Estimations for Certain Increases in the Radius beyond Minimum Values (Segments on Level Grades)

\begin{tabular}{|c|c|c|c|c|c|c|c|c|c|c|c|c|}
\hline \multicolumn{13}{|c|}{ Segments on Level Grades } \\
\hline \multicolumn{13}{|c|}{ Design Speed $=80 \mathrm{~km} / \mathrm{h} ;$ Mimimum Radius $=250 \mathrm{~m}($ Base Case $: C M F=1)$} \\
\hline AADT & \multicolumn{4}{|c|}{1400} & \multicolumn{4}{|c|}{4100} & \multicolumn{4}{|c|}{6600} \\
\hline Def. Angle & 30 & 60 & 90 & 120 & 30 & 60 & 90 & 120 & 30 & 60 & 90 & 120 \\
\hline$R_{\min }$ & 1 & 1 & 1 & 1 & 1 & 1 & 1 & 1 & 1 & 1 & 1 & 1 \\
\hline$R_{\min } \times 1.10$ & $0.998(0.015)$ & $0.995(0.016)$ & $0.989(0.016)$ & $0.987(0.016)$ & $0.997(0.016)$ & $0.992(0.016)$ & $0.988(0.016)$ & $0.986(0.016)$ & $0.996(0.015)$ & 0.991 (0.016) & $0.989(0.016)$ & $0.985(0.016$ \\
\hline$R_{\min } \times 1.25$ & $0.995(0.034)$ & $0.975(0.038)$ & $0.950(0.039)$ & $0.906(0.042)$ & $0.994(0.035)$ & $0.972(0.038)$ & $0.947(0.040)$ & $0.902(0.042)$ & $0.993(0.035)$ & 0.971 (0.039) & $0.946(0.040)$ & $0.900(0.042$ \\
\hline$R_{\min } \times 1.50$ & $0.951(0.058)$ & $0.921(0.064)$ & $0.882(0.083)$ & $0.811(0.103)$ & $0.947(0.057)$ & $0.916(0.065)$ & $0.876(0.085)$ & $0.805(0.111)$ & $0.945(0.058)$ & $0.914(0.068)$ & $0.874(0.086)$ & $0.802(0.111$ \\
\hline$R_{\min } \times 2.00$ & $0.889(0.088)$ & $0.844(0.091)$ & $0.787(0.104)$ & $0.692(0.128)$ & $0.882(0.088)$ & $0.836(0.092)$ & $0.779(0.105)$ & $0.684(0.131)$ & 0.879 (0.089) & $0.833(0.092)$ & $0.776(0.105)$ & $0.681(0.132$ \\
\hline \multicolumn{13}{|c|}{ Design Speed $=90 \mathrm{~km} / \mathrm{h} ;$ Mimimum Radius $=340 \mathrm{~m}($ Base Case: $\mathrm{CMF}=1)$} \\
\hline AADT & \multicolumn{4}{|c|}{1400} & \multicolumn{4}{|c|}{4100} & \multicolumn{4}{|c|}{6600} \\
\hline Def. Angle & 30 & 60 & 90 & 120 & 30 & 60 & 90 & 120 & 30 & 60 & 90 & 120 \\
\hline$R_{\min }$ & 1 & 1 & 1 & 1 & 1 & 1 & 1 & 1 & 1 & 1 & 1 & 1 \\
\hline$R_{\min } \times 1.10$ & $0.979(0.038)$ & $0.966(0.039)$ & $0.952(0.039)$ & $0.924(0.042)$ & $0.978(0.038)$ & $0.965(0.038)$ & $0.950(0.039)$ & $0.922(0.041)$ & $0.978(0.038)$ & $0.965(0.038)$ & $0.949(0.040)$ & $0.921(0.042$ \\
\hline$R_{\min } \times 1.25$ & $0.927(0.062)$ & $0.912(0.068)$ & $0.887(0.070)$ & $0.835(0.072)$ & $0.925(0.063)$ & $0.909(0.068)$ & $0.883(0.071)$ & $0.831(0.073)$ & $0.924(0.063)$ & $0.908(0.068)$ & $0.881(0.072)$ & $0.829(0.073$ \\
\hline$R_{\min } \times 1.50$ & $0.880(0.090)$ & $0.854(0.092)$ & $0.814(0.103)$ & $0.739(0.110)$ & 0.876 (0.091) & 0.849 (0.092) & $0.808(0.104)$ & $0.733(0.112)$ & 0.874 (0.091) & $0.846(0.093)$ & $0.805(0.105)$ & $0.730(0.112$ \\
\hline$R_{\min } \times 2.00$ & $0.798(0.112)$ & $0.764(0.115)$ & $0.711(0.121)$ & $0.619(0.130)$ & $0.791(0.114)$ & $0.756(0.116)$ & $0.703(0.123)$ & $0.612(0.134)$ & $0.788(0.114)$ & $0.753(0.117)$ & $0.700(0.123)$ & $0.608(0.135$ \\
\hline \multicolumn{13}{|c|}{ Design Speed $=100 \mathrm{~km} / \mathrm{h} ;$ Mimimum Radius $=440 \mathrm{~m}$ (Base Case: $\mathrm{CMF}=1)$} \\
\hline AADT & \multicolumn{4}{|c|}{1400} & \multicolumn{4}{|c|}{4100} & \multicolumn{4}{|c|}{6600} \\
\hline Def. Angle & 30 & 60 & 90 & 120 & 30 & 60 & 90 & 120 & 30 & 60 & 90 & 120 \\
\hline$R_{\min }$ & 1 & 1 & 1 & 1 & 1 & 1 & 1 & 1 & 1 & 1 & 1 & 1 \\
\hline$R_{\min } \times 1.10$ & $0.948(0.045)$ & $0.945(0.045)$ & $0.932(0.046)$ & $0.905(0.050)$ & $0.947(0.045)$ & $0.943(0.045)$ & $0.930(0.047)$ & $0.902(0.051)$ & $0.946(0.045)$ & $0.942(0.046)$ & $0.929(0.047)$ & $0.901(0.052$ \\
\hline$R_{\min } \times 1.25$ & $0.892(0.096)$ & $0.884(0.098)$ & $0.859(0.101)$ & $0.806(0.102)$ & 0.889 (0.097) & $0.880(0.100)$ & $0.855(0.102)$ & $0.802(0.102)$ & $0.888(0.097)$ & $0.878(0.101)$ & $0.853(0.102)$ & $0.799(0.103$ \\
\hline$R_{\min } \times 1.50$ & $0.820(0.110)$ & $0.807(0.113)$ & $0.770(0.118)$ & $0.697(0.128)$ & $0.815(0.112)$ & $0.801(0.114)$ & $0.764(0.118)$ & $0.691(0.130)$ & $0.813(0.112)$ & 0.799 (0.116) & $0.762(0.118)$ & $0.688(0.130$ \\
\hline$R_{\min } \times 2.00$ & $0.726(0.126)$ & $0.706(0.131)$ & $0.659(0.134)$ & $0.572(0.141)$ & 0.719 (0.129) & $0.699(0.132)$ & $0.652(0.136)$ & $0.565(0.143)$ & $0.716(0.130)$ & $0.695(0.134)$ & $0.648(0.136)$ & $0.562(0.144$ \\
\hline
\end{tabular}


Table 4.13 Total Crashes CMF Estimations for Certain Increases in the Radius beyond Minimum Values (Segments on Moderate Grades)

\begin{tabular}{|c|c|c|c|c|c|c|c|c|c|c|c|c|}
\hline \multicolumn{13}{|c|}{ Segments on Moderate Grades } \\
\hline \multicolumn{13}{|c|}{ Design Speed $=80 \mathrm{~km} / \mathrm{h} ;$ Mimimum Radius $=250 \mathrm{~m}($ Base Case: $\mathrm{CMF}=1)$} \\
\hline AADT & \multicolumn{4}{|c|}{1400} & \multicolumn{4}{|c|}{4100} & \multicolumn{4}{|c|}{6600} \\
\hline Def. Angle & 30 & 60 & 90 & 120 & 30 & 60 & 90 & 120 & 30 & 60 & 90 & 120 \\
\hline$R_{\min }$ & 1 & 1 & 1 & 1 & 1 & 1 & 1 & 1 & 1 & 1 & 1 & 1 \\
\hline$R_{\min } \times 1.10$ & $0.999(0.016)$ & $0.981(0.017)$ & $0.982(0.016)$ & $0.961(0.019)$ & $0.998(0.016)$ & $0.991(0.016)$ & $0.983(0.016)$ & $0.963(0.018)$ & 0.992 (0.017) & $0.988(0.016)$ & $0.984(0.017)$ & $0.964(0.019)$ \\
\hline$R_{\min } \times 1.25$ & $0.934(0.037)$ & $0.913(0.039)$ & $0.892(0.044)$ & $0.852(0.058)$ & $0.938(0.038)$ & $0.918(0.038)$ & $0.898(0.045)$ & $0.858(0.057)$ & 0.939 (0.038) & $0.920(0.039)$ & $0.900(0.044)$ & $0.861(0.055)$ \\
\hline$R_{\min } \times 1.50$ & $0.839(0.062)$ & $0.826(0.063)$ & $0.801(0.071)$ & $0.745(0.084)$ & $0.847(0.061)$ & $0.835(0.065)$ & $0.810(0.069)$ & $0.755(0.086)$ & $0.851(0.063)$ & $0.839(0.062)$ & $0.814(0.068)$ & $0.760(0.085)$ \\
\hline$R_{\min } \times 2.00$ & $0.739(0.094)$ & $0.725(0.093)$ & $0.694(0.101)$ & $0.625(0.109)$ & $0.751(0.092)$ & $0.737(0.093)$ & $0.706(0.098)$ & $0.638(0.105)$ & $0.756(0.092)$ & $0.743(0.093)$ & $0.712(0.097)$ & $0.643(0.105)$ \\
\hline \multicolumn{13}{|c|}{ Design Speed $=90 \mathrm{~km} / \mathrm{h} ;$ Mimimum Radius $=340 \mathrm{~m}$ (Base Case: $\mathrm{CMF}=1)$} \\
\hline AADT & \multicolumn{4}{|c|}{1400} & \multicolumn{4}{|c|}{4100} & \multicolumn{4}{|c|}{6600} \\
\hline Def. Angle & 30 & 60 & 90 & 120 & 30 & 60 & 90 & 120 & 30 & 60 & 90 & 120 \\
\hline$R_{\min }$ & 1 & 1 & 1 & 1 & 1 & 1 & 1 & 1 & 1 & 1 & 1 & 1 \\
\hline$R_{\min } \times 1.10$ & $0.951(0.032)$ & $0.929(0.035)$ & $0.914(0.036)$ & $0.886(0.042)$ & $0.952(0.031)$ & $0.932(0.035)$ & $0.917(0.038)$ & $0.890(0.040)$ & $0.953(0.032)$ & $0.933(0.034)$ & $0.919(0.038)$ & $0.892(0.041)$ \\
\hline$R_{\min } \times 1.25$ & $0.853(0.077)$ & $0.841(0.078)$ & $0.822(0.084)$ & $0.778(0.091)$ & $0.858(0.077)$ & $0.848(0.077)$ & $0.829(0.084)$ & $0.786(0.090)$ & $0.860(0.076)$ & $0.850(0.077)$ & $0.832(0.083)$ & $0.790(0.088)$ \\
\hline$R_{\min } \times 1.50$ & $0.764(0.096)$ & $0.756(0.096)$ & $0.732(0.101)$ & $0.675(0.105)$ & $0.772(0.095)$ & $0.765(0.096)$ & 0.742 (0.099) & $0.685(0.104)$ & $0.776(0.095)$ & $0.769(0.097)$ & $0.746(0.098)$ & $0.690(0.101)$ \\
\hline$R_{\min } \times 2.00$ & $0.651(0.119)$ & $0.649(0.120)$ & $0.622(0.125)$ & $0.557(0.136)$ & $0.662(0.117)$ & $0.661(0.117)$ & $0.634(0.124)$ & $0.569(0.136)$ & $0.667(0.117)$ & $0.666(0.118)$ & $0.640(0.124)$ & $0.575(0.135)$ \\
\hline \multicolumn{13}{|c|}{ Design Speed $=100 \mathrm{~km} / \mathrm{h} ;$ Mimimum Radius $=440 \mathrm{~m}$ (Base Case: $\mathrm{CMF}=1)$} \\
\hline AADT & \multicolumn{4}{|c|}{1400} & \multicolumn{4}{|c|}{4100} & \multicolumn{4}{|c|}{6600} \\
\hline Def. Angle & 30 & 60 & 90 & 120 & 30 & 60 & 90 & 120 & 30 & 60 & 90 & 120 \\
\hline$R_{\min }$ & 1 & 1 & 1 & 1 & 1 & 1 & 1 & 1 & 1 & 1 & 1 & 1 \\
\hline$R_{\min } \times 1.10$ & $0.901(0.071)$ & 0.899 (0.072) & 0.889 (0.072) & $0.862(0.074)$ & 0.904 (0.069) & $0.903(0.070)$ & $0.893(0.071)$ & $0.867(0.073)$ & 0.905 (0.069) & 0.904 (0.069) & $0.895(0.071)$ & $0.870(0.073)$ \\
\hline $\mathrm{R}_{\min } \times 1.25$ & $0.803(0.103)$ & $0.806(0.103)$ & $0.791(0.106)$ & 0.747 (0.108) & $0.809(0.102)$ & $0.813(0.101)$ & $0.798(0.105)$ & $0.756(0.108)$ & $0.812(0.102)$ & $0.816(0.102)$ & $0.802(0.103)$ & $0.760(0.107)$ \\
\hline$R_{\min } \times 1.50$ & $0.698(0.117)$ & $0.706(0.114)$ & $0.688(0.118)$ & $0.634(0.122)$ & 0.707 (0.114) & $0.716(0.113)$ & $0.698(0.116)$ & 0.645 (0.119) & $0.711(0.114)$ & $0.721(0.112)$ & $0.703(0.115)$ & $0.649(0.119)$ \\
\hline$R_{\min } \times 2.00$ & $0.582(0.134)$ & $0.594(0.133)$ & $0.574(0.136)$ & $0.514(0.141)$ & $0.594(0.131)$ & $0.606(0.131)$ & $0.586(0.135)$ & $0.526(0.139)$ & 0.599 (0.132) & $0.612(0.130)$ & $0.591(0.135)$ & $0.531(0.138)$ \\
\hline
\end{tabular}


Table 4.14 Total Crashes CMF Estimations for Certain Increases in the Radius beyond Minimum Values (Segments on Steep Grades)

\begin{tabular}{|c|c|c|c|c|c|c|c|c|c|c|c|c|}
\hline \multicolumn{13}{|c|}{ Segments on Steep Grades } \\
\hline \multicolumn{13}{|c|}{ Design Speed $=80 \mathrm{~km} / \mathrm{h} ;$ Mimimum Radius $=250 \mathrm{~m}($ Base Case $: C M F=1)$} \\
\hline AADT & \multicolumn{4}{|c|}{1400} & \multicolumn{4}{|c|}{4100} & \multicolumn{4}{|c|}{6600} \\
\hline Def. Angle & 30 & 60 & 90 & 120 & 30 & 60 & 90 & 120 & 30 & 60 & 90 & 120 \\
\hline$R_{\min }$ & 1 & 1 & 1 & 1 & 1 & 1 & 1 & 1 & 1 & 1 & 1 & 1 \\
\hline$R_{\min } \times 1.10$ & $0.985(0.022)$ & $0.974(0.025)$ & $0.941(0.031)$ & $0.916(0.034)$ & $0.989(0.021)$ & $0.975(0.025)$ & $0.944(0.030)$ & $0.920(0.034)$ & $0.991(0.021)$ & $0.975(0.025)$ & $0.945(0.030)$ & $0.922(0.033$ \\
\hline$R_{\min } \times 1.25$ & $0.832(0.049)$ & $0.819(0.051)$ & $0.809(0.058)$ & $0.780(0.062)$ & $0.840(0.047)$ & $0.828(0.050)$ & $0.818(0.058)$ & $0.790(0.060)$ & $0.843(0.047)$ & $0.832(0.050)$ & $0.822(0.057)$ & 0.795 \\
\hline$R_{\min } \times 1.50$ & $0.690(0.083)$ & $0.704(0.081)$ & $0.700(0.082)$ & $0.667(0.085)$ & $0.704(0.082)$ & $0.718(0.079)$ & $0.714(0.079)$ & $0.681(0.085)$ & $0.710(0.082)$ & $0.724(0.079)$ & $0.720(0.078)$ & $0.688(0.084$ \\
\hline$R_{\min } \times 2.00$ & $0.569(0.126)$ & $0.590(0.122)$ & $0.586(0.123)$ & $0.549(0.129)$ & $0.585(0.123)$ & $0.606(0.121)$ & $0.602(0.121)$ & $0.566(0.128)$ & $0.592(0.122)$ & $0.613(0.121)$ & $0.610(0.120)$ & $0.573(0.128$ \\
\hline \multicolumn{13}{|c|}{ Design Speed $=90 \mathrm{~km} / \mathrm{h} ;$ Mimimum Radius $=340 \mathrm{~m}($ Base Case: $\mathrm{CMF}=1)$} \\
\hline AADT & \multicolumn{4}{|c|}{1400} & \multicolumn{4}{|c|}{4100} & \multicolumn{4}{|c|}{6600} \\
\hline Def. Angle & 30 & 60 & 90 & 120 & 30 & 60 & 90 & 120 & 30 & 60 & 90 & 120 \\
\hline$R_{\min }$ & 1 & 1 & 1 & 1 & 1 & 1 & 1 & 1 & 1 & 1 & 1 & 1 \\
\hline$R_{\min } \times 1.10$ & $0.895(0.063)$ & $0.865(0.065)$ & $0.852(0.069)$ & $0.828(0.073)$ & $0.899(0.062)$ & $0.871(0.063)$ & $0.859(0.066)$ & $0.836(0.069)$ & $0.901(0.062)$ & $0.873(0.062)$ & $0.862(0.065)$ & $0.839(0.069$ \\
\hline$R_{\min } \times 1.25$ & 0.739 (0.092) & $0.740(0.092)$ & $0.734(0.094)$ & $0.704(0.098)$ & $0.748(0.091)$ & $0.750(0.091)$ & $0.745(0.092)$ & 0.717 (0.097) & $0.752(0.091)$ & $0.755(0.091)$ & $0.750(0.091)$ & $0.722(0.097)$ \\
\hline$R_{\min } \times 1.50$ & $0.614(0.129)$ & $0.633(0.126)$ & $0.631(0.126)$ & $0.598(0.132)$ & $0.627(0.128)$ & $0.647(0.127)$ & $0.645(0.126)$ & $0.612(0.130)$ & $0.633(0.126)$ & $0.653(0.125)$ & $0.651(0.125)$ & $0.619(0.129$ \\
\hline$R_{\min } \times 2.00$ & $0.489(0.161)$ & $0.518(0.158)$ & $0.519(0.158)$ & $0.486(0.162)$ & $0.503(0.160)$ & $0.534(0.156)$ & $0.535(0.156)$ & $0.501(0.160)$ & $0.510(0.161)$ & $0.540(0.156)$ & $0.542(0.156)$ & $0.508(0.160$ \\
\hline \multicolumn{13}{|c|}{ Design Speed $=100 \mathrm{~km} / \mathrm{h} ;$ Mimimum Radius $=440 \mathrm{~m}($ Base Case: $\mathrm{CMF}=1)$} \\
\hline AADT & \multicolumn{4}{|c|}{1400} & \multicolumn{4}{|c|}{4100} & \multicolumn{4}{|c|}{6600} \\
\hline Def. Angle & 30 & 60 & 90 & 120 & 30 & 60 & 90 & 120 & 30 & 60 & 90 & 120 \\
\hline$R_{\min }$ & 1 & 1 & 1 & 1 & 1 & 1 & 1 & 1 & 1 & 1 & 1 & 1 \\
\hline$R_{\min } \times 1.10$ & $0.815(0.082)$ & $0.821(0.082)$ & $0.819(0.083)$ & $0.798(0.085)$ & $0.822(0.082)$ & $0.829(0.081)$ & $0.827(0.081)$ & $0.807(0.083)$ & $0.825(0.081)$ & $0.833(0.081)$ & $0.831(0.082)$ & $0.811(0.083$ \\
\hline$R_{\min } \times 1.25$ & $0.672(0.138)$ & $0.696(0.134)$ & $0.697(0.134)$ & $0.670(0.139)$ & $0.683(0.136)$ & $0.708(0.133)$ & $0.709(0.133)$ & $0.683(0.138)$ & $0.688(0.136)$ & $0.713(0.132)$ & $0.715(0.132)$ & $0.688(0.138$ \\
\hline$R_{\min } \times 1.50$ & $0.546(0.157)$ & $0.582(0.151)$ & $0.586(0.149)$ & $0.556(0.152)$ & $0.560(0.154)$ & $0.596(0.149)$ & $0.600(0.148)$ & $0.571(0.150)$ & $0.566(0.154)$ & $0.602(0.148)$ & $0.607(0.147)$ & $0.577(0.149$ \\
\hline$R_{\min } \times 2.00$ & $0.427(0.182)$ & $0.468(0.179)$ & $0.473(0.178)$ & $0.443(0.180)$ & $0.441(0.180)$ & $0.483(0.178)$ & $0.489(0.176)$ & $0.459(0.177)$ & $0.448(0.180)$ & $0.489(0.177)$ & $0.495(0.176)$ & $0.465(0.175$ \\
\hline
\end{tabular}


Similar to segments on level grades, it can be seen from Table 4.13, that CMF estimates for segments on moderate grades follow a similar trend irrespective of the AADT. For example, at a design speed of $80 \mathrm{~km} / \mathrm{h}$, the CMF for increasing the radius by a factor 1.50 at deflection angle of $90^{\circ}$ is $\sim 0.700$ irrespective of the AADT. Similarly, at a design speed of $100 \mathrm{~km} / \mathrm{h}$, the CMF for increasing the radius by a factor of 2 at deflection angle of $30^{\circ}$ is $\sim 0.590$, irrespective of the AADT. It can also be seen that even at different speeds, the CMF estimates for a certain scenario seem to be following a similar trend.

Similar to the results on level and moderate grades, it can be seen from Table 4.14, that the CMF estimates for segments on steep grades follow a similar trend irrespective of AADT and that at different speeds, the estimates for a certain scenario seem to be following a similar trend.

Table 4.15 summarizes the average approximate crash reduction rate (derived from Tables 4.12, 4.13 and 4.14) irrespective of the design speeds and AADTs for a specified increase in radius.

These results are comparable to those found by Zegeer et al. (1992) and Hauer (1999). They concluded that the greater the curve flattening is, the higher is the reduction in crashes is. Our results show an average crash reduction of between $20 \%$ - 55\% for an increase in radius by a factor of 2 over all three grade categories, compared to an average crash reduction of between $15 \%-45 \%$ for an increase in radius by factor of 1.50 over all three grade categories. These results are reasonably consistent with the average crash reduction of $35 \%-55 \%$ found by Zegeer et al. (1992) for increasing the radius by a factor of 2 . 
Table 4.15 Average Crash Reduction Rate (CRR) from the Results in Table 4-12 - 4-14

\begin{tabular}{|c|c|c|c|c|}
\hline \multicolumn{5}{|c|}{ Segments on Level Grades } \\
\hline Def. Angle & 30 & 60 & 90 & 120 \\
\hline$C R R$ for $R_{\min } \times 1.10$ & $1 \%-5 \%$ & $1 \%-5 \%$ & $1 \%-10 \%$ & $1 \%-10 \%$ \\
\hline$C R R$ for $R_{\min } \times 1.25$ & $1 \%-15 \%$ & $1 \%-15 \%$ & $5 \%-15 \%$ & $10 \%-20 \%$ \\
\hline$C R R$ for $R_{\min } \times 1.50$ & $5 \%-20 \%$ & $10 \%-20 \%$ & $15 \%-25 \%$ & $20 \%-30 \%$ \\
\hline$C R R$ for $R_{\min } \times 2.00$ & $15 \%-30 \%$ & $15 \%-30 \%$ & $20 \%-35 \%$ & $30 \%-45 \%$ \\
\hline \multicolumn{5}{|c|}{ Segments on Moderate Grades } \\
\hline Def. Angle & 30 & 60 & 90 & 120 \\
\hline$C R R$ for $R_{\min } \times 1.10$ & $1 \%-5 \%$ & $1 \%-10 \%$ & $1 \%-10 \%$ & $1 \%-15 \%$ \\
\hline$C R R$ for $R_{\min } \times 1.25$ & $5 \%-20 \%$ & $5 \%-20 \%$ & $10 \%-20 \%$ & $15 \%-25 \%$ \\
\hline$C R R$ for $R_{\min } \times 1.50$ & $15 \%-30 \%$ & $15 \%-30 \%$ & $20 \%-35 \%$ & $25 \%-35 \%$ \\
\hline$C R R$ for $R_{\min } \times 2.00$ & $25 \%-40 \%$ & $25 \%-40 \%$ & $30 \%-45 \%$ & $35 \%-50 \%$ \\
\hline \multicolumn{5}{|c|}{ Segments on Steep Grades } \\
\hline Def. Angle & 30 & 60 & 90 & 120 \\
\hline$C R R$ for $R_{\min } \times 1.10$ & $1 \%-15 \%$ & $1 \%-20 \%$ & $1 \%-20 \%$ & $1 \%-20 \%$ \\
\hline$C R R$ for $R_{\min } \times 1.25$ & $15 \%-35 \%$ & $15 \%-30 \%$ & $25 \%-30 \%$ & $25 \%-35 \%$ \\
\hline$C R R$ for $R_{\min } \times 1.50$ & $30 \%-45 \%$ & $30 \%-40 \%$ & $30 \%-40 \%$ & $30 \%-45 \%$ \\
\hline$C R R$ for $R_{\min } \times 2.00$ & $40 \%-55 \%$ & $40 \%-55 \%$ & $40 \%-55 \%$ & $45 \%-55 \%$ \\
\hline
\end{tabular}

The results also show a higher crash reduction for segments on higher grades, for e.g. increasing the radius by a factor of 2 at a deflection angle of $120^{\circ}$ has an average crash reduction of $45 \%$ - $55 \%$ on steep grades compared to $35 \%-50 \%$ on moderate grades.

The results shown in Tables $4.12-4.15$ also support Hauer's (1999) argument in that the CMF estimates for a certain scenario are approximately the same (i.e. they lie in the same average range) irrespective of the value of radius chosen as the base case.

To further validate the results, Figure 4.8, 4.9 and 4.10 show analogous plots of F\&I CMFs where the length of the horizontal curve, shoulder width and percent grade were kept constant and the radius of the horizontal curve varied. To be consistent, in all cases, the minimum radius of $250 \mathrm{~m}$ for design speeds of $80 \mathrm{~km} / \mathrm{h}$ was used as the base case to derive the CMFs for 
increasing the radius by a factor of 1.10, 1.25, 1.50, 2.00 and 3.00 at an AADT of 4100 (the $50^{\text {th }}$ percentile of the dataset). The main purpose here is to compare the CMFs for F\&I crashes derived from Tables 4.6-4.11 with the CMFs for the same scenarios as would be derived using Equation 4-2 from Bauer and Harwood (2013).

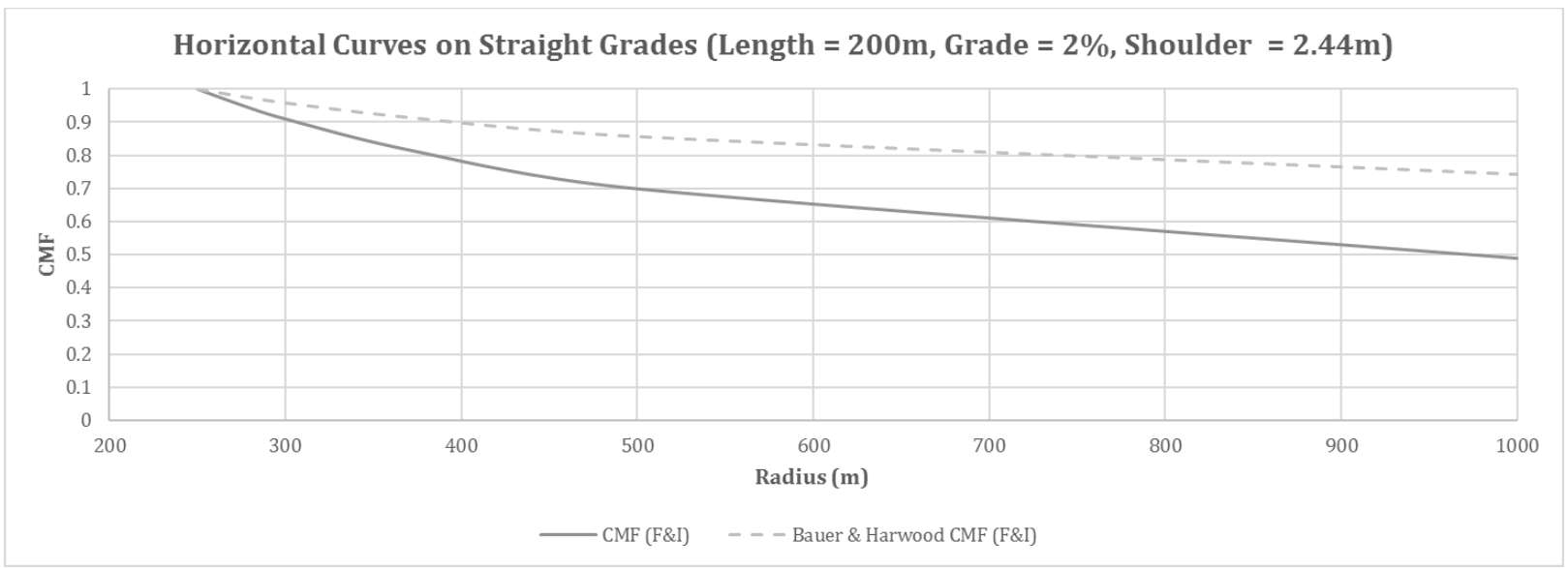

Figure 4.8 Comparison of CMFs for Varying Radii on Level Grade (2\%)

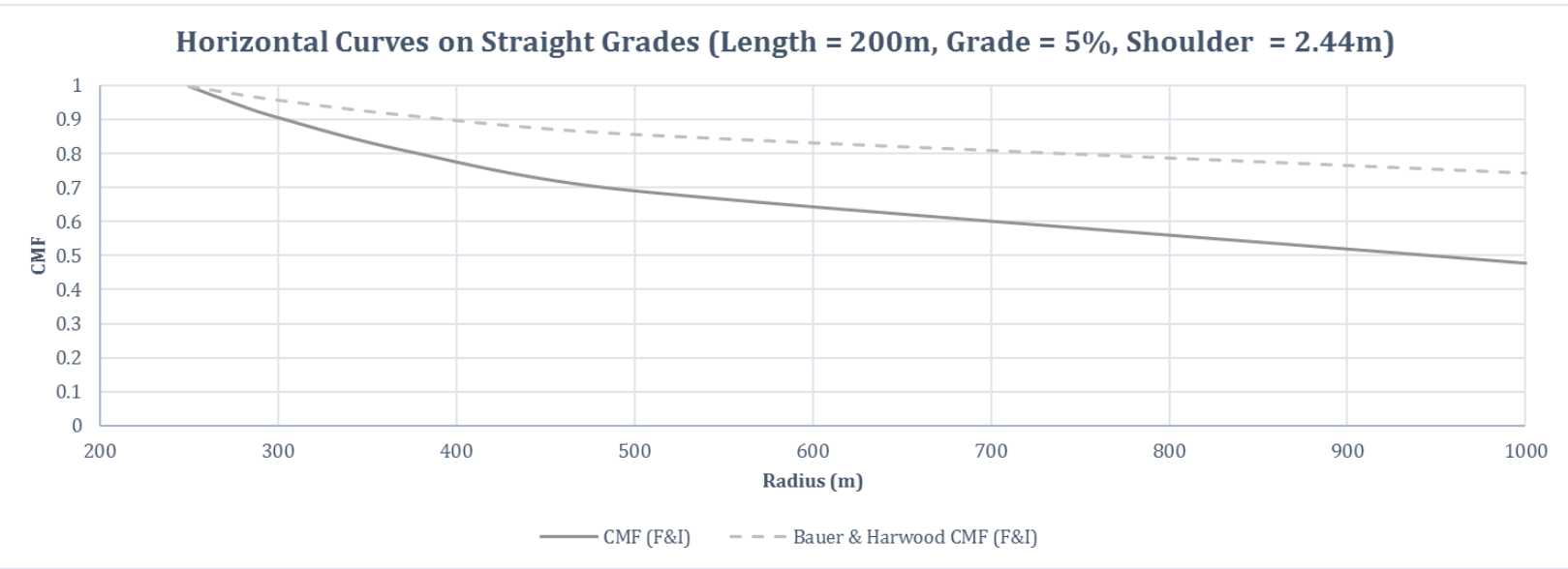

Figure 4.9 Comparison of CMFs for Varying Radii on Moderate Grade (5\%) 


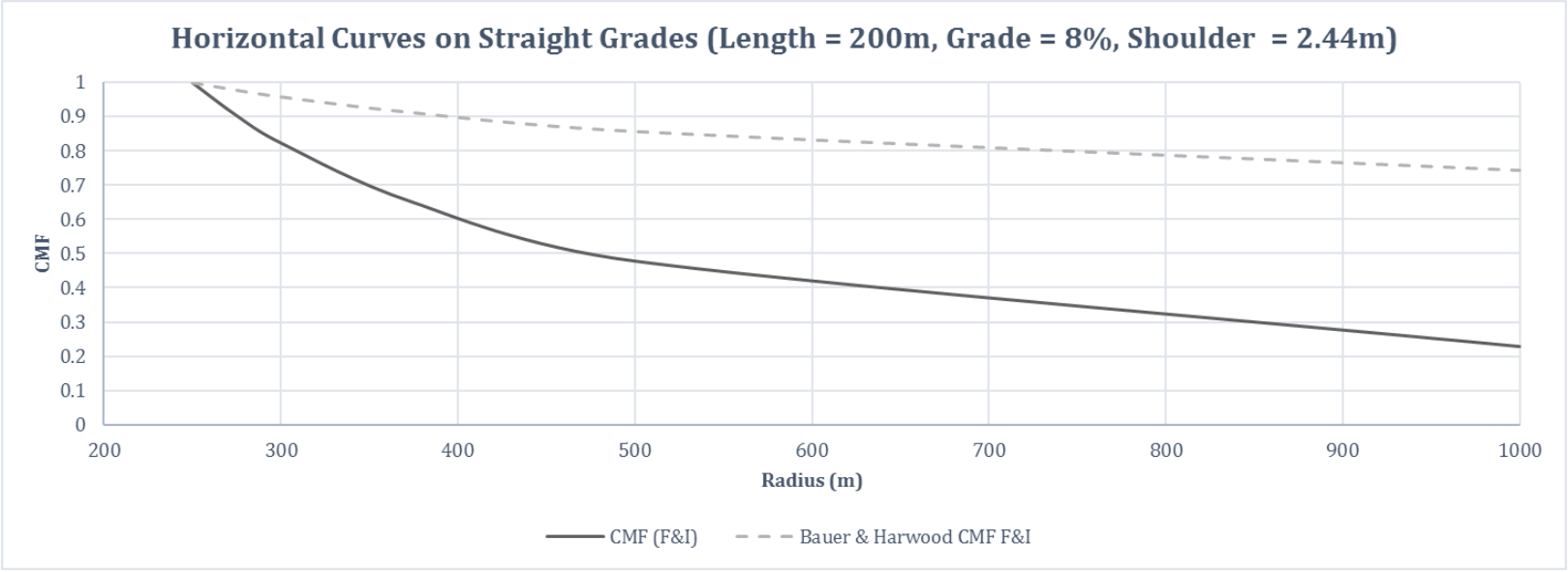

Figure 4.10 Comparison of CMFs for Varying Radii on Steep Grade (8\%)

The plots (Figures $4.8-4.10$ ) shows that the CMFs for F\&I crashes developed in this dissertation (Tables $4.6-4.11$ ) are consistently larger than the CMFs that would materialize under the model developed by Bauer \& Harwood (2013). One of the main reason for this trend can be the fact that Bauer \& Harwood (2013) in their study grouped all the horizontal curves (irrespective of their percent grade), whereas, for the purpose of this dissertation the horizontal curves were grouped into three categories; level grades $(\leq 3 \%)$, moderate grades $(3-6 \%)$, and steep grades $(>6 \%)$, so that the CMFs for changing horizontal curvature could be explored without the confounding effects of gradient.

\section{$\underline{4.5 \text { Chapter Summary }}$}

This chapter provides an assessment of the potential of cross-sectional analysis to derive CMFs in the absence of before-after data. In doing so, new insights were provided into estimating CMFs for flattening an existing horizontal curve on rural 2-lane highways. Crash prediction models were developed for the curve sections and also for the tangents up and down stream of the curve sections on level $(<3 \%)$, moderate $(3 \%-6 \%)$, and steep $(>6 \%)$ grades. These models 
were statistically significant to the 5\% level and had low standard errors. The goodness of prediction measures also indicated a good fit.

To further demonstrate the capabilities of this approach, the relationship between crashes at different volumes and deflection angles was explored to get approximate estimates of CMFs for increasing the minimum radius required by a factor of $1.10,1.25,1.50$, and 2.00 . In this case, to accurately compare crashes between the old curve and the flattened curve, crashes on the tangent section that was removed to accommodate the longer length of the flattened curve were also taken into account. The results show that even at different design speeds, the CMF estimates for a certain scenario lie in the same range conforming to the findings from Zegeer et al. (1992) and Hauer (1999). Furthermore, the CMFs for F\&I crashes were also plotted against the CMFs from Bauer and Harwood (2013). The results here showed that the effect of changing horizontal curvature was captured as being much larger when the data were explored without the confounding effects of gradient. These results show that cross-sectional analysis can be used effectively to capture the effect of a treatment in cases where before-after data is either not available or is impossible to obtain. 


\section{Chapter 5. Evaluating the Potential of Cross-Sectional}

\section{Analysis to Develop CMFs and CMFunctions using Before-}

\section{After Study Databases}

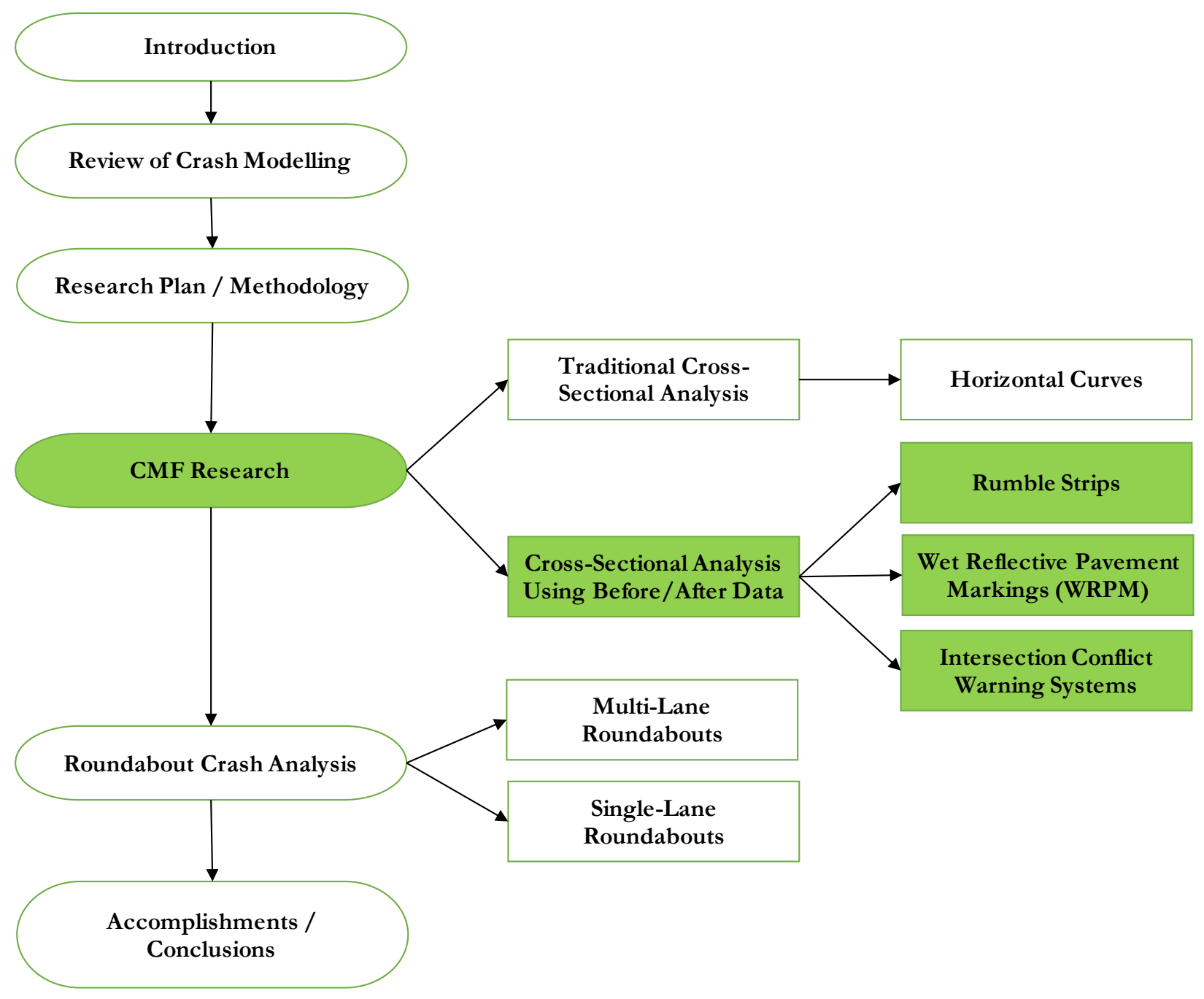

This chapter focuses on the second part of the CMF research component in this dissertation -evaluating the consistency in the estimation of CMFs from cross-sectional analysis using actual before-after databases. The main purpose of this evaluation is to investigate whether 
cross-sectional analysis can produce comparable results to before-after analysis and to examine the potential of cross-sectional analysis to better develop CMFunctions even in the presence of before-after data. The first part of the chapter presents the first case study in this evaluation using combination (center line plus shoulder) rumble strips as a design element, while the second part presents a case study for wet reflective pavement markings, and the third part presents a case study for intersection conflict warning systems.

The data used came from Minnesota, North Carolina, Kentucky, Missouri, Pennsylvania, and Wisconsin Departments of Transportation. The data, which were used in recent empirical Bayes before-after studies (Persaud et al. (2016), Lyon et al. (2016), and Himes et al. (2016)), included both treatment sites (where combination rumble strips, wet reflective pavement markings, and intersection conflict warning systems were installed, with the installation dates) and reference sites (similar to treated sites in characteristics but without the treatment). It is unclear how the reference sites were chosen, but, ideally, reference sites need to be chosen carefully to be deemed similar in characteristics to the treatment sites in the before period. Tools such as propensity score matching can be used to ensure that the two groups are matched equally and that any differences between the treatment and control groups are not a result of differences on the matching variables (Thavaneswaran \& Lix, 2008). Donnell et al. (2011) presented a comparison of inverse propensity score weighting and propensity score matching in the context of data used for transportation safety studies.

A cross-sectional analysis was conducted using negative binomial regression and a combination of the reference site data and the after period data at the treatment sites to derive functions that can subsequently be used to estimate CMFs. These CMFs were then compared 
to those from recent studies, where the above mentioned treatments were evaluated using empirical Bayes before-after analysis with the datasets used for the cross-sectional regression.

The main motivation for this part of the research is to answer three questions: (1) Can the effect of treatment be inferred given the problems with cross-sectional analysis? (2) Can crosssectional analysis provide CMF estimates comparable to the CMF estimates from before-after analysis? and (3) Can cross-sectional analysis better estimate CMFunctions?

\section{$\underline{\text { 5.1 Safety Evaluation of Combination Rumble Strips }}$}

This section presents the first case study in this investigation -- using combination (center line plus shoulder) rumble strips as a design element and data from a before-after study by Persaud et al. (2016).

\subsubsection{Background/Motivation}

Rumble strips are milled grooves placed along the center line and/or shoulder of a roadway. They are a low cost treatment for reducing crashes related to veering off the lane way such as run off road, head on and sideswipe opposite direction crashes (Persaud et al., 2016).

Shoulder rumble strips may be placed on the edge line or offset some distance into a paved shoulder. Center line rumble strips are similar in principle to shoulder rumble strips. The purpose is to alert drivers who may inadvertently stray or encroach into opposing lanes. Although there is no standard design, these rumble strips are generally wider than the center markings, extending into the travel lane by $12.7 \mathrm{~cm}(5 \mathrm{in}$.) to as much as $0.46 \mathrm{~m}(1.5 \mathrm{ft})$. (Persaud et al., 2016). 
Several research studies have been conducted to assess the safety performance of either center line or shoulder rumble strips, but as Persaud et al. (2016) concluded after reviewing them, research assessing the combined effect of center line and shoulder rumble strips at the time of their study has been rare and limited in scope. As a result, the Highway Safety Manual (AASTHO, 2010) does not provide a CMF for the combined effect of center line plus shoulder rumble strips.

Perhaps the most relevant and robust study was one done by Sayed et al. (2010) evaluated the safety impacts of applying center line and shoulder rumble strips at a combination two-lane and four-lane rural highways in British Columbia, Canada. They conducted an empirical Bayes before-after using 47 treated sites and 225 comparison sites. Their results showed that the installation of the combined center line and shoulder rumble strips can reduce total crashes by 18\%. A higher reduction of $21.4 \%$ was seen for run off road and head on crashes. Another study by Kay et al. (2015) assessed the safety impacts of the statewide center line and edge line rumble strips implemented by the Michigan Department of Transportation (Michigan DOT) between the years of 2008-2010. The program was meant to blanket cover more than $8,000 \mathrm{~km}$ of rural non-freeway highways maintained by Michigan DOT. Edge line rumble strips were installed in combination with centerline rumble strips in areas where paved shoulders were at least $1.83 \mathrm{~m}$ (6 ft.). Using the empirical Bayes methodology, they found a $27.3 \%$ reduction at center line rumble strips sites and a $32.8 \%$ reduction at sites with combination center line and edge line rumble strips for target crashes (crashes involving vehicles crossing the centerline). Another study conducted by Torbic et al. (2013) also looked at evaluating the combined effect of center line and edge line rumble strips in the state of 
Mississippi. They looked at two-lane rural highways and defined their target crashes as head on, sideswipe opposite direction and run off road. Their results showed a reduction of $35 \%$ in total crashes and $39.6 \%$ in fatal/injury crashes at sites where the treatment was applied.

The main objective of this section is to explore the development of crash modification function (CMFunction) to estimate CMFs for application of combined center line plus shoulder rumble strips. For this purpose, crash prediction models were developed using using negative binomial regression and a combination of the reference site data and the after period data at the treatment sites to derive functions. The CMFs estimated from the parameters of these models are then compared those from the study by Persaud et al. (2016), where center line plus shoulder rumble strips were evaluated using empirical Bayes before-after analysis with the datasets used for the cross-sectional regression.

\subsubsection{Summary of Data}

The data for this study were provided by Kentucky and Pennsylvania Department's of Transportation and consisted of two-lane rural roads. The data consisted of both the locations and dates of the installation of center line and shoulder rumble strips alongside information on road geometry, volumes and crashes for both reference and installation sites.

Tables 5.1 and 5.2 show some summary statistics and crash data for the Kentucky sites. The data consisted of $264 \mathrm{~km}$ of treated sites and $2440 \mathrm{~km}$ of reference sites with 11 years of crash data (ranging from 2002-2012) available. The speed limit at all the Kentucky sites used was $55 \mathrm{mi} . / \mathrm{hr}(\sim 90 \mathrm{~km} / \mathrm{hr})$. 
Table 5.1 Summary Statistics for Kentucky Sites

\begin{tabular}{|c|ccc|}
\hline \multicolumn{4}{|c|}{ Kentucky Treatment Sites $(\mathrm{n}=27)$} \\
\hline Variable & Minimum & Maximum & Average \\
\hline AADT & 1290.00 & 20433.00 & 6166.21 \\
Length $(m)$ & 804.67 & 26100.27 & 9760.76 \\
Shoulder $(m)$ & 0.61 & 3.66 & 2.50 \\
\hline \multicolumn{5}{|c|}{ Kentucky Reference Sites $(\mathrm{n}=988)$} \\
\hline Variable & Minimum & Maximum & Average \\
\hline AADT & 10.00 & 18200.00 & 2723.26 \\
\hline Length $(m)$ & 14.84 & 20710.60 & 2469.93 \\
\hline Shoulder $(m)$ & 0.00 & 4.27 & 1.87 \\
\hline
\end{tabular}

Note: $1 \mathrm{mi}=1609.34 \mathrm{~m}, 1 \mathrm{ft}$. $=0.3048 \mathrm{~m}$

Table 5.2 Crash Statistics for Kentucky Sites

\begin{tabular}{|c|c|c|c|}
\hline \multicolumn{5}{|c|}{ Kentucky Treatment Sites ( $\mathbf{n = 2 7 )}$} \\
\hline Crash Type & Minimum & Maximum & Average \\
\hline Total & 1 & 128 & 26.63 \\
Injury & 0 & 29 & 7.78 \\
Run off Road & 0 & 22 & 5.52 \\
Head-On & 0 & 3 & 0.56 \\
Side-Swipe Opposite-Direction & 0 & 4 & 1.15 \\
\hline \multicolumn{5}{|c|}{ Kentucky Reference Sites } & (n = 988) \\
\hline Crash Type & Minimum & Maximum & Average \\
\hline Total & 0 & 355 & 18.16 \\
Injury & 0 & 125 & 5.83 \\
\hline Run off Road & 0 & 170 & 6.43 \\
Head-On & 0 & 12 & 0.54 \\
\hline
\end{tabular}

Tables 5.3 and 5.4 show some summary statistics and crash data for the Pennsylvania sites. The data consisted of $351 \mathrm{~km}$ of treated sites and $28,856 \mathrm{~km}$ of reference sites with 10 years of crash data (ranging from 2003-2012) available. The speed limit at all the Pennsylvania sites used was between $35-55 \mathrm{mi} . / \mathrm{hr}(\sim 55-90 \mathrm{~km} / \mathrm{hr})$. 
Table 5.3 Summary Statistics for Pennsylvania Sites

\begin{tabular}{|c|c|c|c|}
\hline \multicolumn{4}{|c|}{ Pennsylvania Treatment Sites $(n=464)$} \\
\hline Variable & Minimum & Maximum & Average \\
\hline AADT & 745.00 & 25706.00 & 4920.15 \\
\hline Length $(m)$ & 18.25 & 1213.92 & 755.94 \\
\hline Shoulder (m) & 0.00 & 3.05 & 1.40 \\
\hline \multicolumn{4}{|c|}{ Pennsylvania Treatment Sites $(n=39360)$} \\
\hline Variable & Minimum & Maximum & Average \\
\hline$A A D T$ & 470.00 & 26173.00 & 4325.06 \\
\hline Length $(m)$ & 15.24 & 2029.05 & 733.15 \\
\hline Shoulder (m) & 0.00 & 4.88 & 0.94 \\
\hline
\end{tabular}

Note: $1 \mathrm{mi}=1609.34 \mathrm{~m}, 1 \mathrm{ft}$. $=0.3048 \mathrm{~m}$

Table 5.4 Crash Statistics for Pennsylvania Sites

\begin{tabular}{|c|c|c|c|}
\hline \multicolumn{4}{|c|}{ Pennsylvania Treatment Sites ( $\mathrm{n}=464$ ) } \\
\hline Crash Type & Minimum & Maximum & Average \\
\hline Total & 0 & 23 & 1.24 \\
Injury & 0 & 9 & 0.68 \\
Run offRoad & 0 & 7 & 0.19 \\
Head-On & 0 & 2 & 0.05 \\
Side-Swipe Opposite-Direction & 0 & 3 & 0.03 \\
\hline \multicolumn{4}{|c|}{ Pennsylvania Treatment Sites (n=39360) } \\
\hline Crash Type & Minimum & Maximum & Average \\
\hline Total & 0 & 107 & 5.59 \\
Injury & 0 & 60 & 2.92 \\
Run off Road & 0 & 35 & 1.01 \\
Head-On & 0 & 17 & 0.24 \\
\hline
\end{tabular}

\subsubsection{Model Fitting and Evaluation}

Consistent with state-of-the-art methods, generalized linear modelling, with the specification of a negative binomial (NB) error structure (as discussed in Section 3.4), was used to develop the crash prediction models (Persaud et al., 2012) using the SAS software (SAS, 2016). 


\subsubsection{Model Fitting Using Kentucky Data}

Two models were developed using the Kentucky data. The first form modelled the presence of center line plus shoulder rumble strips yielding a crash modification factor for the presence of the combination rumble strips. The second form looked at the interaction between the presence of center line and shoulder rumble strips and the shoulder width. This would lead to a crash modification function where the magnitude of crash reduction is not only based on the presence of the combination rumble strips, but also depends on the shoulder width.

\subsection{Modelling the Presence of Combination Rumble Strips - Model A}

The model form used for developing the models incorporating the presence of combination rumble strips was as follows:

Crashes $/$ Km $/$ Year $=e^{\alpha} \times A A D T^{\beta_{1}} \times e^{\beta_{2} \times R S}$

Where;

$A A D T=$ Average annual daily traffic, and

$R S=$ Presence of center line plus shoulder rumble strips: 1 if present, 0 if not present.

Tables 5.5 and 5.6 show the coefficient estimates, over dispersion parameters $(\mathrm{K})$ and the goodness of prediction measures for models for various crash severities. 
Table 5.5 Estimates and Dispersion Parameters for Kentucky Model A

\begin{tabular}{|c|c|c|c|c|}
\hline Crash Type & \multicolumn{2}{|l|}{ Total } & \multicolumn{2}{|l|}{ Injury } \\
\hline Coefficient & $\begin{array}{c}\text { Estimate } \\
\text { (Standard Error) }\end{array}$ & Pr>Chisq & $\begin{array}{c}\text { Estimate } \\
\text { (Standard Error) }\end{array}$ & Pr>Chisq \\
\hline$\alpha$ & $-5.0256(0.2746)$ & $<0.0001$ & $-5.6673(0.3041)$ & $<0.0001$ \\
\hline 81 & $0.6126(0.0356)$ & $<0.0001$ & 0.5389 (0.0389) & $<0.0001$ \\
\hline 82 & $-0.2832(0.2026)$ & 0.1622 & $-0.3374(0.2106)$ & 0.1091 \\
\hline$K$ & \multicolumn{2}{|l|}{0.8973} & \multicolumn{2}{|l|}{0.7326} \\
\hline Crash Type & \multicolumn{2}{|c|}{ Run Off Road } & \multicolumn{2}{|c|}{ Head On } \\
\hline Coefficient & $\begin{array}{c}\text { Estimate } \\
\text { (Standard Error) }\end{array}$ & Pr>Chisq & $\begin{array}{c}\text { Estimate } \\
\text { (Standard Error) }\end{array}$ & Pr>ChiSq \\
\hline$\alpha$ & $-4.2793(0.3264)$ & $<0.0001$ & $-8.4602(0.5787)$ & $<0.0001$ \\
\hline 81 & $0.3774(0.0424)$ & $<0.0001$ & 0.5820 (0.0719) & $<0.0001$ \\
\hline 82 & $-0.6143(0.2456)$ & 0.0124 & $-0.8125(0.3570)$ & 0.0229 \\
\hline$K$ & \multicolumn{2}{|l|}{0.9372} & \multicolumn{2}{|l|}{0.7729} \\
\hline Crash Type & \multicolumn{2}{|c|}{ Side Swipe (OD) } & & \\
\hline Coefficient & $\begin{array}{c}\text { Estimate } \\
\text { (Standard Error) }\end{array}$ & Pr>Chisq & & \\
\hline$\alpha$ & $-6.4539(0.4758)$ & $<0.0001$ & & \\
\hline 81 & $0.4049(0.0602)$ & $<0.0001$ & & \\
\hline 82 & $-0.3269(0.3081)$ & 0.2887 & & \\
\hline$K$ & 0.8759 & & & \\
\hline
\end{tabular}

Table 5.6 Goodness of Prediction Measures for Kentucky Model A

\begin{tabular}{|c|c|c|}
\hline \multicolumn{2}{|c|}{ Crash Type } & Total \\
\hline Avg. Obs. Crashes/Year/Site & 18.3901 & 5.8807 \\
MAD/Year/Site & 0.5559 & 0.1938 \\
MPE/Year/Site & 0.1094 & 0.0341 \\
\hline Crash Type & Run Off Road & Head On \\
\hline Avg. Obs. Crashes/Year/Site & 6.4009 & 0.5428 \\
MAD/Year/Site & 0.2327 & 0.0282 \\
MPE/Year/Site & 0.0415 & 0.0055 \\
\hline Crash Type & Side Swipe (OD) & \\
\hline Avg. Obs. Crashes/Year/Site & 0.9310 & \\
MAD/Year/Site & 0.0493 & \\
MPE/Year/Site & 0.0088 & \\
\hline
\end{tabular}

As can be seen from Tables 5.5 and 5.6, the estimates of $\beta_{1}$ are highly significant $(\mathrm{P}<0.01)$ for all the models. Estimates for $\beta_{2}$ are significant to the $15 \%$ level for all the crashes except for the sideswipe opposite direction crashes. Even though the $\beta_{2}$ estimate for sideswipe opposite direction crashes was not significant, the model can still be considered good because the direction of the magnitude of $\beta_{2}$ is correct suggesting of a decrease in crashes when the 
combination rumble strips are present. The goodness of prediction measures also suggests of reasonably good fits in that the MAD/year/site (Mean Absolute Deviation) and the MPE/year/site (Mean Prediction Error) for all the models are small (close to zero in all cases) when compared to the average observed crashes per year per site. For example, for total crashes the MAD/year/site is 0.556 and MPE/year/site is 0.109 compared to an average of $~ 18.39$ total crashes per year per site. Similarly, for run off road crashes, the MAD/year/site is 0.233 and MPE/year/site is 0.042 compared to an average of $\sim 6.40$ run off road crashes per year per site. The CURE plots for all models are shown in Figures 5.1.
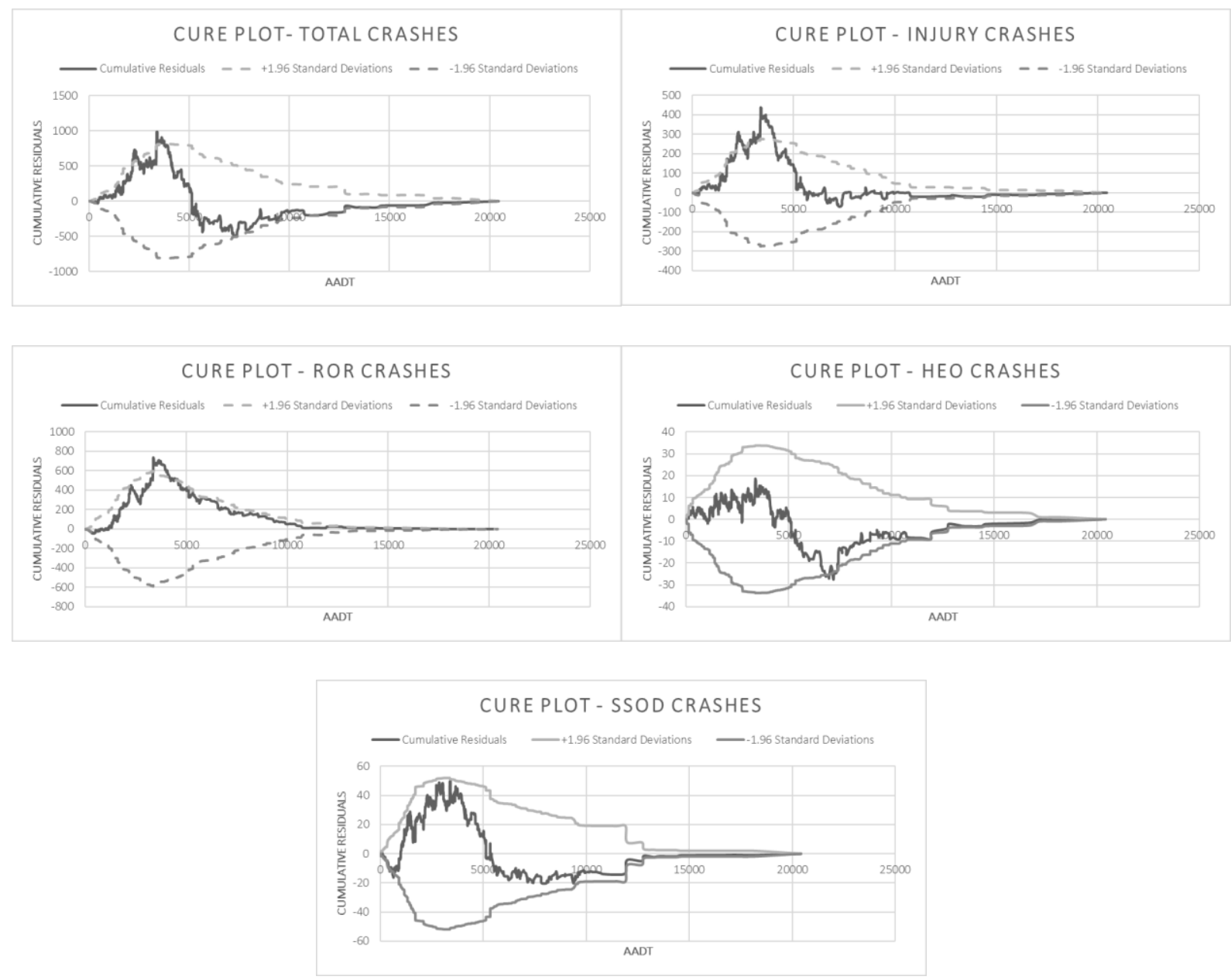

Figure 5.1 Cure Plots for Kentucky Model A 
The CURE plots for all models, shown in Figure 5.1, indicate that for total and injury crashes, the CURE plot does oscillate consistently but at two points a steep vertical jump takes it outside of the $95 \%$ confidence boundaries. This can be indicative of possible outliers (Hauer, 2014). The plot for run off road crashes shows a large section of increasing runs followed by a large section of decreasing runs. Though the plot stays within the $95 \%$ confidence boundaries at most times, this behavior is indicative of consistent over/under prediction by the model (Hauer, 2014). The plots for head on and sideswipe opposite direction crashes do oscillate consistently and stay within the $95 \%$ confidence boundaries suggesting of a good fit.

Overall, based on all the goodness of prediction measures characterized by very low MAD/year/site and MPE/year/site values, and CURE plots staying within the 95\% confidence boundaries, it can be said that the models developed are well fit and can predict crashes reasonably close to reality.

\subsection{Modelling the Interaction of Combination Rumble Strips with Shoulder Width - Model B}

The model form used for developing the models incorporating the interaction between the presence of combination rumble strips and the shoulder width was as follows:

Crashes $/$ Km $/$ Year $=e^{\alpha} \times A A D T^{\beta_{1}} \times e^{\beta_{2} \times(\text { Shoulder Width } \times R S)}$

Where;

$A A D T=$ Average annual daily traffic,

Shoulder Width = Shoulder width in meters, and 
$R S=$ Presence of center line plus shoulder rumble strips: 1 if present, 0 if not present.

Incorporating the interaction between shoulder width and rumble strips will allow the crash reduction rate to vary with the varying shoulder widths.

Tables 5.7 and 5.8 show the coefficient estimates, over dispersion parameters $(\mathrm{K})$ and the goodness of prediction measures for models for various crash severities. CURE plots for all models are shown in Figure 5.2.

Table 5.7 Estimates and Dispersion Parameters for Kentucky Model B

\begin{tabular}{|c|c|c|c|c|}
\hline Crash Type & \multicolumn{2}{|l|}{ Total } & \multicolumn{2}{|l|}{ Injury } \\
\hline Coefficient & $\begin{array}{c}\text { Estimate } \\
\text { (Standard Error) }\end{array}$ & Pr>ChiSq & $\begin{array}{c}\text { Estimate } \\
\text { (Standard Error) }\end{array}$ & Pr>Chisq \\
\hline$\alpha$ & $-5.0487(0.2743)$ & $<0.0001$ & $-5.6775(0.3032)$ & $<0.0001$ \\
\hline 81 & $0.6159(0.0355)$ & $<0.0001$ & $0.5404(0.0388)$ & $<0.0001$ \\
\hline 82 & $-0.1572(0.0746)$ & 0.0351 & $-0.1535(0.0731)$ & 0.0354 \\
\hline$K$ & \multicolumn{2}{|l|}{0.8946} & \multicolumn{2}{|l|}{0.7298} \\
\hline Crash Type & \multicolumn{2}{|c|}{ Run Off Road } & \multicolumn{2}{|c|}{ Head On } \\
\hline Coefficient & $\begin{array}{c}\text { Estimate } \\
\text { (Standard Error) }\end{array}$ & Pr>ChiSq & $\begin{array}{c}\text { Estimate } \\
\text { (Standard Error) }\end{array}$ & Pr>ChiSq \\
\hline$\alpha$ & $-4.3122(0.3252)$ & $<0.0001$ & $-8.4706(0.5774)$ & $<0.0001$ \\
\hline 81 & $0.3823(0.0422)$ & $<0.0001$ & $0.5836(0.0718)$ & $<0.0001$ \\
\hline 82 & $-0.3162(0.0820)$ & 0.0001 & $-0.3003(0.1147)$ & 0.0089 \\
\hline$K$ & \multicolumn{2}{|l|}{0.9297} & \multicolumn{2}{|l|}{0.7674} \\
\hline Crash Type & \multicolumn{2}{|c|}{ Side Swipe (OD) } & & \\
\hline Coefficient & $\begin{array}{c}\text { Estimate } \\
\text { (Standard Error) }\end{array}$ & Pr>Chisq & & \\
\hline$\alpha$ & $-6.5148(0.4745)$ & $<0.0001$ & & \\
\hline 81 & $0.4137(0.0600)$ & $<0.0001$ & & \\
\hline 82 & $-0.2238(0.1001)$ & 0.0253 & & \\
\hline$K$ & 0.8636 & & & \\
\hline
\end{tabular}


Table 5.8 Goodness of Prediction Measures for Kentucky Model B

\begin{tabular}{|c|c|c|}
\hline \multicolumn{2}{|c|}{ Crash Type } & Total \\
\hline Avg. Obs. Crashes/Year/Site & 18.3901 & 5.8807 \\
MAD/Year/Site & 0.5557 & 0.1933 \\
MPE/Year/Site & 0.1089 & 0.0340 \\
\hline Crash Type & Run Off Road & Head On \\
\hline Avg. Obs. Crashes/Year/Site & 6.4009 & 0.5428 \\
MAD/Year/Site & 0.2328 & 0.0283 \\
MPE/Year/Site & 0.0414 & 0.0055 \\
\hline Crash Type & Side Swipe (OD) & \\
\hline Avg. Obs. Crashes/Year/Site & 0.9310 & \\
MAD/Year/Site & 0.0493 & \\
MPE/Year/Site & 0.0088 & \\
\hline
\end{tabular}
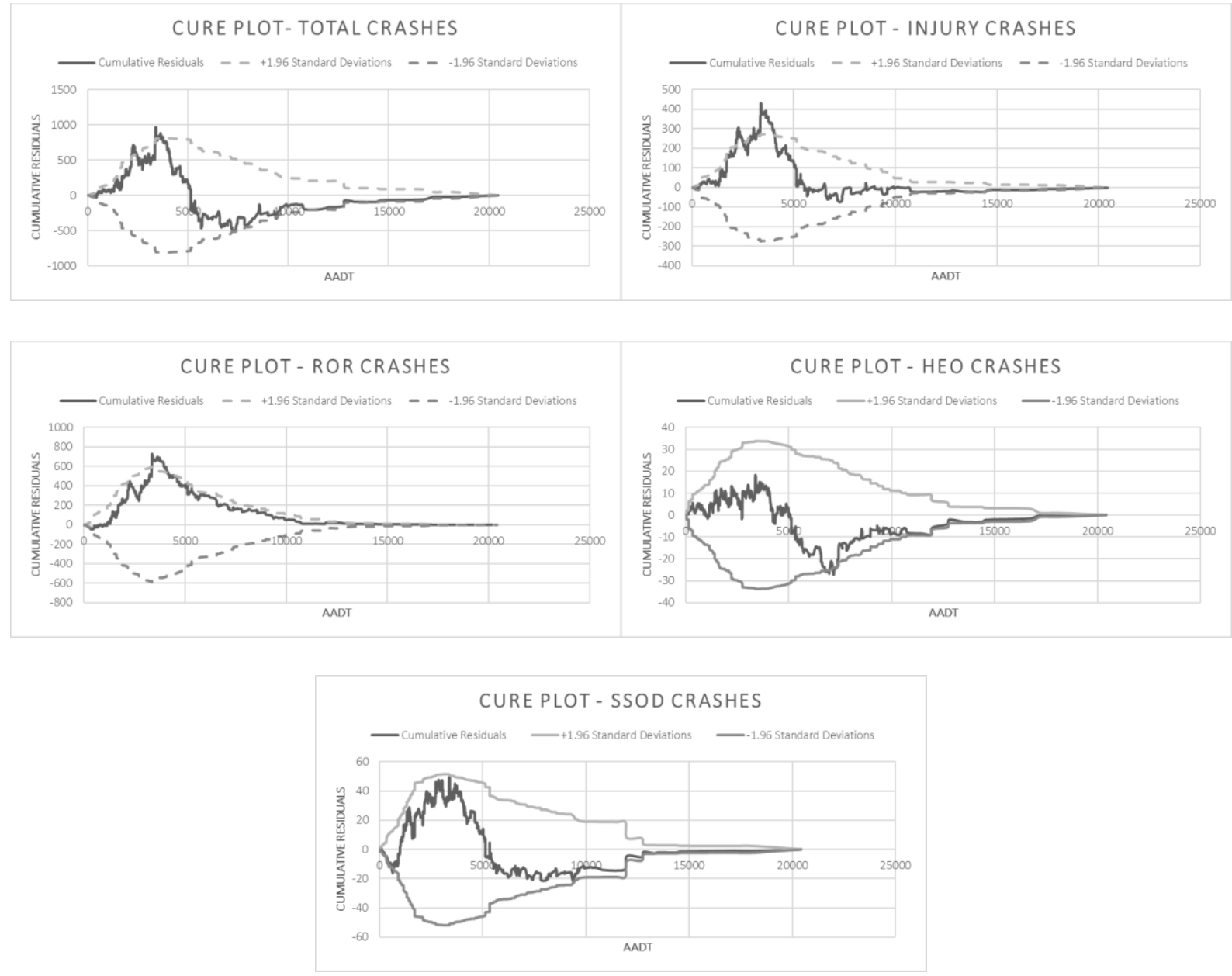

Figure 5.2 Cure Plots for Kentucky Model B 
As can be seen from Tables 5.7 and 5.8, the estimates of $\beta_{1}$ and $\beta_{2}$ are highly significant $(\mathrm{P}<0.05)$ for all the models. The goodness of prediction measures also suggests of reasonably good fits in that the MAD/year/site (Mean Absolute Deviation) and the MPE/year/site (Mean Prediction Error) for all the models are small (close to zero in almost all cases) when compared to the average observed crashes per year per site. For example, for total crashes the MAD/year/site is 0.5557 and MPE/year/site is 0.1089 compared to an average of 18.39 total crashes per year per site. Similarly, for run off road crashes, the MAD/year/site is 0.2328 and $\mathrm{MPE} / \mathrm{year} / \mathrm{site}$ is 0.0414 compared to an average of $\sim 6.40$ run off road crashes per year per site.

The CURE plots for all models, shown in Figure 5.2, show similar characteristics to the CURE plots for Model A shown in Figure 5.1. It can be seen that for total and injury crashes, the CURE plot does oscillate consistently but at two points a steep vertical jump takes it outside of the $95 \%$ confidence boundaries. This can be indicative of possible outliers (Hauer, 2014). The plot for run off road crashes shows a large section of increasing runs followed by a large section of decreasing runs. Though the plot stays within the $95 \%$ confidence boundaries at most times, this behavior is indicative of consistent over/under prediction by the model (Hauer, 2014). The plots for head on and sideswipe opposite direction crashes do oscillate consistently and stay within the $95 \%$ confidence boundaries suggesting a reasonably good fit.

Overall, based on all the goodness of prediction measures characterized by very low MAD/year/site and MPE/year/site values and CURE plots staying within the 95\% confidence boundaries it can be said that the models developed are well fit and can predict crashes reasonably close to reality. 


\subsubsection{Model Fitting Using Pennsylvania Data}

Similar to the Kentucky models, two models were developed using the Pennsylvania data, the first modelling the presence of center line plus shoulder rumble strips and the second modelling the interaction between the presence of center line and shoulder strips and the shoulder width.

\subsection{Modelling the Presence of Combination Rumble Strips -Model A}

The model form used for developing the models incorporating the presence of combination rumble strips and the shoulder width was as follows:

Crashes $/$ Km $/$ Year $=e^{\alpha} \times A A D T^{\beta_{1}} \times e^{\beta_{2} \times R S}$

Where;

$A A D T=$ Average annual daily traffic, and

$R S=$ Presence of center line plus shoulder rumble strips: 1 if present, 0 if not present.

Tables 5.9 and 5.10 show the coefficient estimates, over dispersion parameters $(\mathrm{K})$ and the goodness of prediction measures for models for various crash severities. CURE plots for all models are shown in Figure 5.3. 
Table 5.9 Estimates and Dispersion Parameters for Pennsylvania Model A

\begin{tabular}{|c|c|c|c|c|}
\hline Crash Type & \multicolumn{2}{|l|}{ Total } & \multicolumn{2}{|l|}{ Injury } \\
\hline Coefficient & $\begin{array}{c}\text { Estimate } \\
\text { (Standard Error) }\end{array}$ & Pr>ChiSq & $\begin{array}{c}\text { Estimate } \\
\text { (Standard Error) }\end{array}$ & Pr>ChiSq \\
\hline$\alpha$ & $-6.2675(0.0382)$ & $<0.0001$ & $-7.0884(0.0438)$ & $<0.0001$ \\
\hline 81 & $0.7294(0.0047)$ & $<0.0001$ & $0.7487(0.0053)$ & $<0.0001$ \\
\hline 82 & $-0.2156(0.0554)$ & 0.0001 & $-0.1174(0.0674)$ & 0.0816 \\
\hline$K$ & \multicolumn{2}{|l|}{0.4413} & \multicolumn{2}{|l|}{0.4345} \\
\hline Crash Type & \multicolumn{2}{|c|}{ Run Off Road } & \multicolumn{2}{|c|}{ Head On } \\
\hline Coefficient & $\begin{array}{c}\text { Estimate } \\
\text { (Standard Error) }\end{array}$ & Pr>ChiSq & $\begin{array}{c}\text { Estimate } \\
\text { (Standard Error) }\end{array}$ & Pr>Chisq \\
\hline$\alpha$ & $-5.9997(0.0689)$ & $<0.0001$ & $-10.8243(0.1178)$ & $<0.0001$ \\
\hline 81 & $0.4940(0.0084)$ & $<0.0001$ & 0.8919 (0.0137) & $<0.0001$ \\
\hline 82 & $-0.2797(0.1167)$ & 0.0165 & $-0.1697(0.1073)$ & 0.2129 \\
\hline$K$ & \multicolumn{2}{|l|}{0.9616} & \multicolumn{2}{|l|}{0.7210} \\
\hline Crash Type & \multicolumn{2}{|c|}{ Side Swipe (OD) } & & \\
\hline Coefficient & $\begin{array}{c}\text { Estimate } \\
\text { (Standard Error) }\end{array}$ & Pr>Chisq & & \\
\hline$\alpha$ & $-10.5451(0.1432)$ & $<0.0001$ & & \\
\hline 81 & 0.7999 (0.0167) & $<0.0001$ & & \\
\hline 82 & $-0.2411(0.1228)$ & 0.1768 & & \\
\hline$K$ & 0.7490 & & & \\
\hline
\end{tabular}

Table 5.10 Goodness of Prediction Measures for Pennsylvania Model A

\begin{tabular}{|c|c|c|}
\hline \multicolumn{2}{|c|}{ Crash Type } & Total \\
\hline Avg. Obs. Crashes/Year/Site & 5.5381 & 2.9032 \\
MAD/Year/Site & 0.6176 & 0.3512 \\
MPE/Year/Site & 0.0110 & 0.0062 \\
\hline Crash Type & Run Off Road & Head On \\
\hline Avg. Obs. Crashes/Year/Site & 0.9969 & 0.2382 \\
MAD/Year/Site & 0.1771 & 0.0535 \\
MPE/Year/Site & 0.0032 & 0.0011 \\
\hline Crash Type & Side Swipe (OD) & \\
\hline Avg. Obs. Crashes/Year/Site & 0.1425 & \\
MAD/Year/Site & 0.0366 & \\
MPE/Year/Site & 0.0008 & \\
\hline
\end{tabular}

As can be seen from Tables 5.9 and 5.10, the estimates of $\beta_{1}$ and $\beta_{2}$ are significant for all the models. Estimates for $\beta_{3}$ in some cases come up to be insignificant (for head on and side swipe opposite direction crashes). Even though the $\beta_{3}$ estimates for head on and sideswipe opposite direction crashes was not significant, the models can still be considered good because the 
direction of the magnitude of $\beta_{3}$ is correct suggesting of a decrease in crashes when the combination rumble strips are present. Thus, the insignificance in the estimates can be explained by the small number of treatment sites; 464 compared to 39360 reference sites and also by the low average/year/site occurrence of these two types of crashes. The goodness of prediction measures also suggests of reasonably good fits in that the MAD/year/site (Mean Absolute Deviation) and the MPE/year/site (Mean Prediction Error) for all the models are small (close to zero in all cases) when compared to the average observed crashes per year per site. For example, for total crashes the MAD/year/site is 0.613 and MPE/year/site is 0.011 compared to an average of $\sim 5.54$ total crashes per year per site. Similarly, for run off road crashes, the MAD/year/site is 0.177 and MPE/year/site is 0.003 compared to an average of $\sim 1.00$ run off road crash per year per site.

The CURE plots for all models are shown in Figure 5.3. The CURE plots show that for the range of AADT between $\sim 6000$ and $\sim 19000$, the cumulative residuals lie outside the $95 \%$ confidence boundaries, in almost all cases, suggesting of a bias in the predictions in this AADT range. This can very well be indicative of long periods of over/under prediction by these models (Hauer, 2014). The CURE plots for head on and sideswipe opposite direction crashes do oscillate consistently suggesting of a better fit compared to other models. However, CURE plots alone cannot be used to judge the significance of the models and since the other goodness of prediction measures do suggest of a good overall fit, we can say that overall these models can predict crashes reasonably well. 

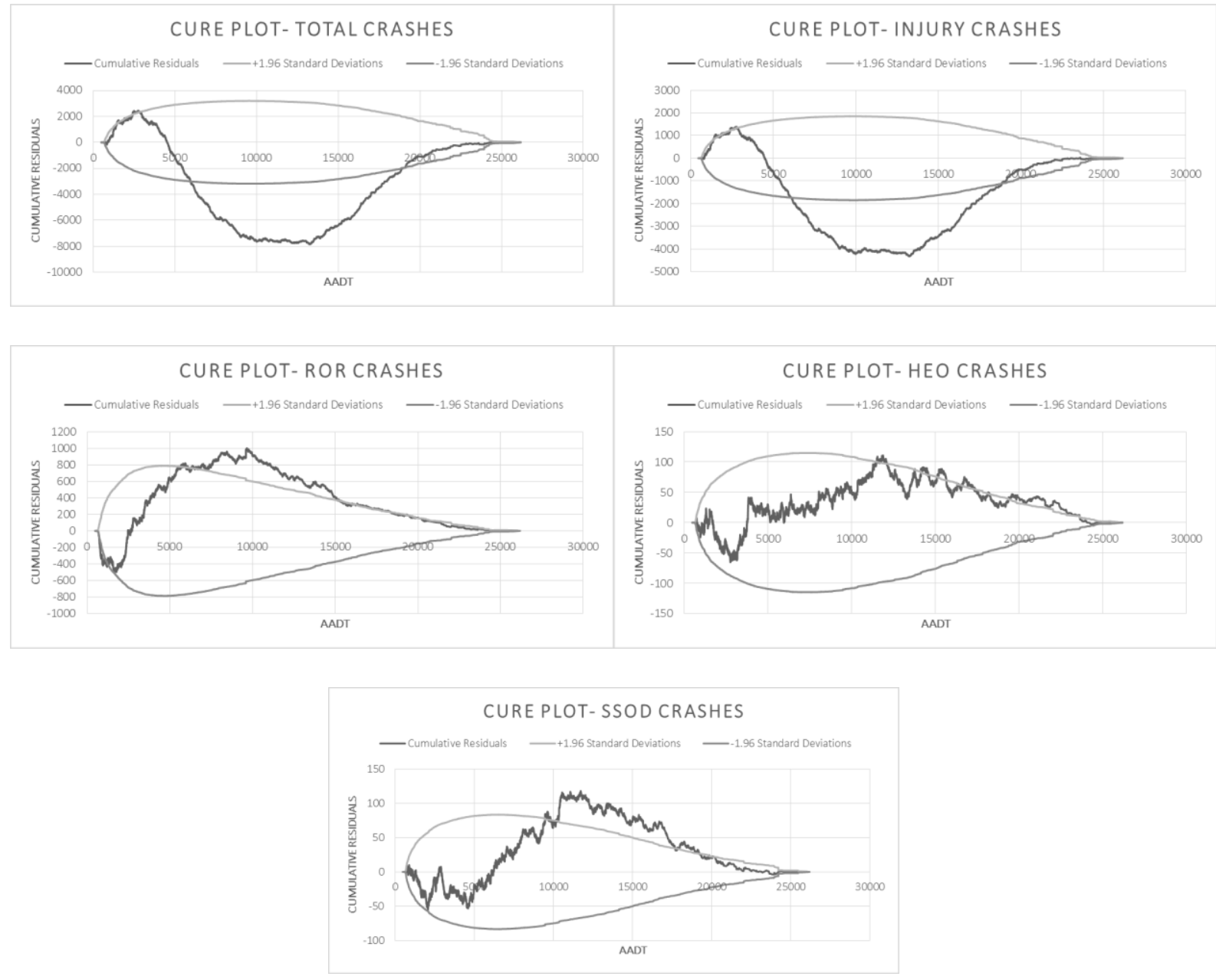

Figure 5.3 CURE Plots for Pennsylvania Model A

\subsection{Modelling the Interaction of Combination Rumble Strips with Shoulder Width - Model B}

The model form used for developing the models incorporating the interaction between the presence of combination rumble strips and the shoulder width was as follows:

Crashes $/$ Km $/$ Year $=e^{\alpha} \times A A D T^{\beta_{1}} \times e^{\beta_{2} \times(\text { ShoulderWidth } \times R S)}$

Where; 
$A A D T=$ Average annual daily traffic,

Shoulder Width $=$ Shoulder width in meters, and

$R S=$ Presence of center line plus shoulder rumble strips: 1 if present, 0 if not present.

Tables 5.11 and 5.12 show the coefficient estimates, over dispersion parameters (K) and the goodness of prediction measures for models for various crash severities. CURE plots for all models are shown in Figure 5.4.

As can be seen from Tables 5.11 and 5.12, the estimates of $\beta_{1}$ and $\beta_{2}$ are highly significant $(\mathrm{P}<0.01)$ for all models. Estimates for $\beta_{3}$, like Model A, are seen to be insignificant for head on and sideswipe opposite direction crashes, but the direction of the magnitude suggests of the correct effect. This could once again be due the very small number of treatment sites compared to reference sites and the low occurrence/year/site of the head on and sideswipe opposite direction crashes. The goodness of prediction measures also suggests of reasonably good fits in that the MAD/year/site (Mean Absolute Deviation) and the MPE/year/site (Mean Prediction Error) for all the models are small (close to zero in all cases) when compared to the average observed crashes per year per site. For example, for total crashes the MAD/year/site is 0.613 and MPE/year/site is 0.011 compared to an average of $\sim 5.54$ total crashes per year per site. 
Table 5.11 Estimates and Dispersion Parameters for Pennsylvania Model B

\begin{tabular}{|c|c|c|c|c|}
\hline Crash Type & \multicolumn{2}{|l|}{ Total } & \multicolumn{2}{|l|}{ Injury } \\
\hline Coefficient & $\begin{array}{c}\text { Estimate } \\
\text { (Standard Error) }\end{array}$ & Pr>Chisq & $\begin{array}{c}\text { Estimate } \\
\text { (Standard Error) }\end{array}$ & Pr>Chisq \\
\hline$\alpha$ & $-6.2700(0.0382)$ & $<0.0001$ & $-7.0901(0.0438)$ & $<0.0001$ \\
\hline 81 & $0.7297(0.0047)$ & $<0.0001$ & $0.7489(0.0053)$ & $<0.0001$ \\
\hline 82 & $-0.1371(0.0352)$ & $<0.0001$ & $-0.0965(0.0431)$ & 0.0253 \\
\hline$K$ & \multicolumn{2}{|l|}{0.4413} & \multicolumn{2}{|l|}{0.4344} \\
\hline Crash Type & \multicolumn{2}{|c|}{ Run Off Road } & \multicolumn{2}{|c|}{ Head On } \\
\hline Coefficient & $\begin{array}{c}\text { Estimate } \\
\text { (Standard Error) }\end{array}$ & Pr>ChiSq & $\begin{array}{c}\text { Estimate } \\
\text { (Standard Error) }\end{array}$ & Pr>ChiSq \\
\hline$\alpha$ & $-6.0027(0.0690)$ & $<0.0001$ & $-10.8248(0.1178)$ & $<0.0001$ \\
\hline 81 & $0.4944(0.0084)$ & $<0.0001$ & $0.8919(0.0137)$ & $<0.0001$ \\
\hline 82 & $-0.2665(0.0833)$ & 0.0014 & $-0.0489(0.0385)$ & 0.3798 \\
\hline$K$ & \multicolumn{2}{|l|}{0.9615} & \multicolumn{2}{|l|}{0.7210} \\
\hline Crash Type & \multicolumn{2}{|c|}{ Side Swipe (OD) } & & \\
\hline Coefficient & $\begin{array}{c}\text { Estimate } \\
\text { (Standard Error) }\end{array}$ & Pr>ChiSq & & \\
\hline$\alpha$ & $-10.5464(0.1432)$ & $<0.0001$ & & \\
\hline 81 & $0.8000(0.0167)$ & $<0.0001$ & & \\
\hline 82 & $-0.1122(0.1016)$ & 0.3274 & & \\
\hline$K$ & 0.7490 & & & \\
\hline
\end{tabular}

Table 5.12 Goodness of Prediction Measures for Pennsylvania Model B

\begin{tabular}{|c|c|c|}
\hline \multicolumn{2}{|c|}{ Crash Type } & Total \\
\hline Avg. Obs. Crashes/Year/Site & 5.5381 & 2.9032 \\
MAD/Year/Site & 0.6176 & 0.3511 \\
MPE/Year/Site & 0.0110 & 0.0062 \\
\hline Crash Type & Run Off Road & Head On \\
\hline Avg. Obs. Crashes/Year/Site & 0.9969 & 0.2382 \\
MAD/Year/Site & 0.177 & 0.0535 \\
MPE/Year/Site & 0.0032 & 0.0011 \\
\hline Crash Type & Side Swipe (OD) & \\
\hline Avg. Obs. Crashes/Year/Site & 0.1425 & \\
MAD/Year/Site & 0.0366 & \\
MPE/Year/Site & 0.0008 & \\
\hline
\end{tabular}



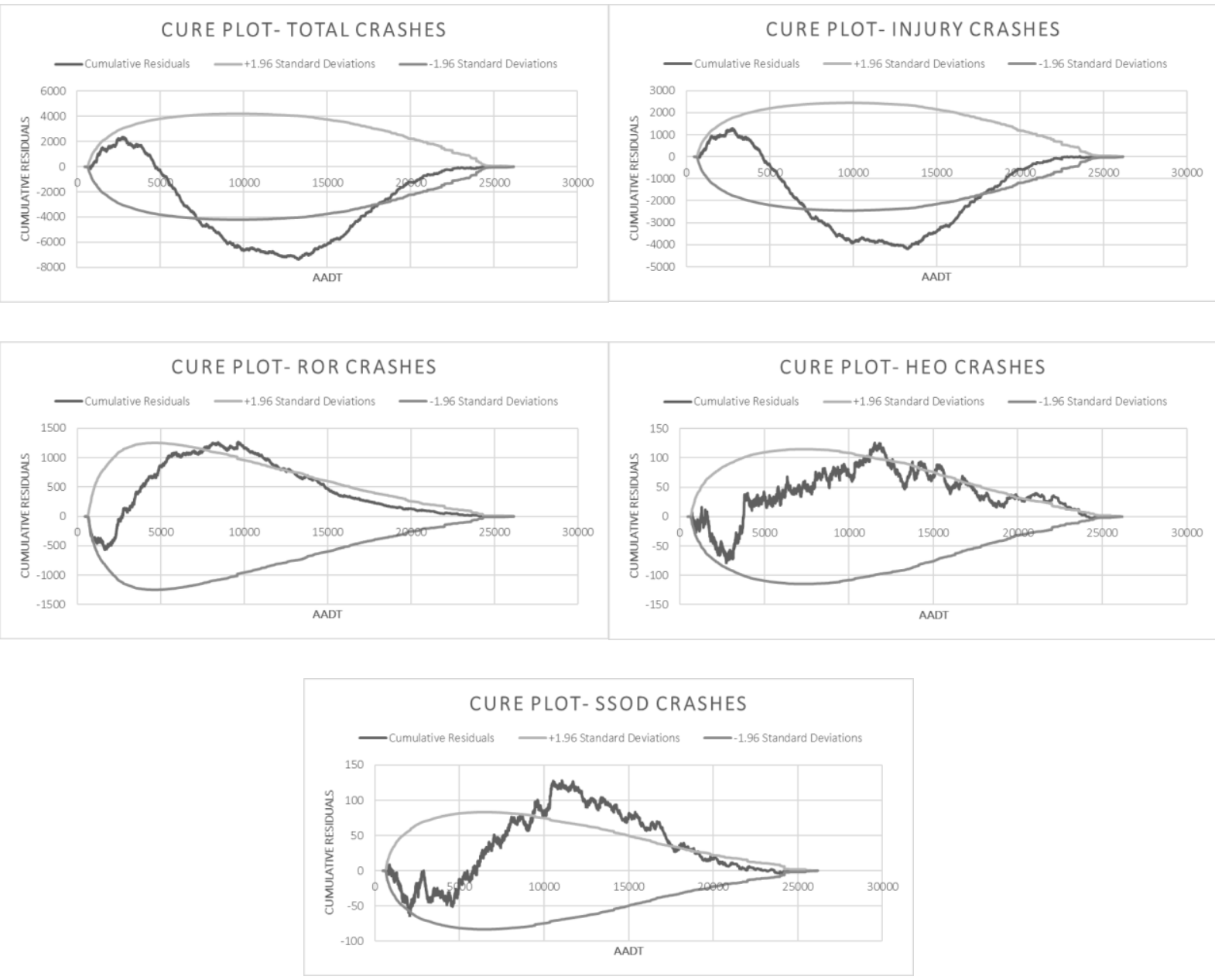

Figure 5.4 CURE Plots for Pennsylvania Model B

The CURE plots (Figure 5.4) show a similar trend to the CURE plots for Model A shown in Figure 5.3 in that for the range of AADT between $\sim 6000$ and $\sim 19000$, the cumulative residuals lie outside the $95 \%$ confidence boundaries, in almost all cases, suggesting of a bias in the predictions in this AADT range. This can once again be indicative of long periods of over/under prediction by these models (Hauer, 2014). The CURE plots for head on and sideswipe opposite direction crashes do oscillate consistently suggesting of a better fit compared to other models. However, CURE plot alone cannot be used to judge the significance 
of the models and since the other goodness of prediction measures do suggest of a good overall fit, we can say that overall these models can predict crashes reasonably well.

\subsubsection{Model Fitting Using Combined (Kentucky + Pennsylvania) Data}

Since the models developed for Kentucky and Pennsylvania showed insignificant estimates for head on and sideswipe opposite direction crashes, an attempt was made to develop models by combining both datasets. Similar to the previous sections, two models were developed using the combined data, the first modelling the presence of center line plus shoulder rumble strips and the second modelling the interaction between the presence of center line and shoulder rumble strips and the shoulder width.

\subsection{Modelling the Presence of Combination Rumble Strips - Model A}

The model form used for developing the models incorporating the presence of combination rumble strips and the shoulder width was as follows:

Crashes $/$ Km $/$ Year $=e^{\alpha+\beta_{3}} \times A A D T^{\beta_{1}} \times e^{\beta_{2} \times R S}$

Where;

$A A D T=$ Average annual daily traffic, and

$R S=$ Presence of center line plus shoulder rumble strips: 1 if present, 0 if not present.

Tables 5.13 and 5.14 show the coefficient estimates, over dispersion parameters (K) and the goodness of prediction measures for models for various crash severities. CURE plots for all models are shown in Figure 5.5. 
As can be seen from Tables 5.13 and 5.14, the estimates of $\beta_{1}, \beta_{2}$ and $\beta_{3}$ are highly significant $(<0.01)$ in almost all the cases. The goodness of prediction measures also suggests of reasonably good fits in that the MAD/year/site (Mean Absolute Deviation) and the MPE/year/site (Mean Prediction Error) for all the models are small (close to zero in almost all cases) when compared to the average observed crashes per year per site. For example, for total crashes the MAD/year/site is 0.616 and MPE/year/site is 0.011 compared to an average of $\sim 5.86$ total crashes per year per site. Similarly, for run off road crashes, the MAD/year/site is 0.178 and MPE/year/site is 0.003 compared to an average of $\sim 1.13$ run off road crash per year per site.

Table 5.13 Estimates and Dispersion Parameters for Combined Model A

\begin{tabular}{|c|c|c|c|c|}
\hline \multirow{2}{*}{$\begin{array}{l}\text { Crash Type } \\
\text { Coefficient }\end{array}$} & \multicolumn{2}{|l|}{ Total } & \multicolumn{2}{|l|}{ Injury } \\
\hline & $\begin{array}{c}\text { Estimate } \\
\text { (Standard Error) }\end{array}$ & Pr>Chisq & $\begin{array}{c}\text { Estimate } \\
\text { (Standard Error) }\end{array}$ & Pr>ChiSa \\
\hline$\alpha$ & $-6.2398(0.0381)$ & $<0.0001$ & $-7.0436(0.0435)$ & $<0.0001$ \\
\hline 81 & $0.7261(0.0046)$ & $<0.0001$ & $0.7433(0.0052)$ & $<0.0001$ \\
\hline 82 & $-0.2363(0.0527)$ & $<0.0001$ & $-0.1701(0.0643)$ & 0.0081 \\
\hline 83 (Kentucky) & $0.3076(0.0269)$ & $<0.0001$ & $-0.2466(0.0313)$ & $<0.0001$ \\
\hline 83 (Pennsylvania) & 0 & - & 0 & - \\
\hline$K$ & \multicolumn{2}{|l|}{0.4551} & \multicolumn{2}{|l|}{0.4448} \\
\hline Crash Type & \multicolumn{2}{|c|}{ Run Off Road } & \multicolumn{2}{|c|}{ Head On } \\
\hline Coefficient & $\begin{array}{c}\text { Estimate } \\
\text { (Standard Error) }\end{array}$ & Pr>ChiSq & $\begin{array}{c}\text { Estimate } \\
\text { (Standard Error) }\end{array}$ & Pr>ChiSq \\
\hline$\alpha$ & $-5.9627(0.0676)$ & $<0.0001$ & $-10.7338(0.1158)$ & $<0.0001$ \\
\hline 81 & $0.4894(0.0082)$ & $<0.0001$ & $0.8814(0.0135)$ & $<0.0001$ \\
\hline 82 & $-0.3554(0.1089)$ & 0.0011 & $-0.4081(0.1909)$ & 0.0325 \\
\hline B3 (Kentucky) & $0.8205(0.0400)$ & $<0.0001$ & $-0.1528(0.0628)$ & 0.0149 \\
\hline B3 (Pennsylvania) & 0 & - & 0 & - \\
\hline$K$ & \multicolumn{2}{|l|}{0.9615} & \multicolumn{2}{|l|}{0.7309} \\
\hline Crash Type & \multicolumn{2}{|c|}{ Side Swipe (OD) } & & \\
\hline Coefficient & $\begin{array}{c}\text { Estimate } \\
\text { (Standard Error) }\end{array}$ & Pr>Chisq & & \\
\hline$\alpha$ & $-10.3181(0.1385)$ & $<0.0001$ & & \\
\hline 81 & $0.7733(0.0162)$ & $<0.0001$ & & \\
\hline 82 & $-0.4128(0.2121)$ & 0.0517 & & \\
\hline B3 (Kentucky) & $0.9492(0.0572)$ & $<0.0001$ & & \\
\hline B3 (Pennsylvania) & 0 & - & & \\
\hline$K$ & 0.8079 & & & \\
\hline
\end{tabular}


Table 5.14 Goodness of Prediction Measures for Combined Model A

\begin{tabular}{|c|c|c|}
\hline \multicolumn{2}{|c|}{ Crash Type } & Total \\
\hline Avg. Obs. Crashes/Year/Site & 5.8576 & 2.9772 \\
MAD/Year/Site & 0.6161 & 0.3472 \\
MPE/Year/Site & 0.0111 & 0.0061 \\
\hline Crash Type & Run Off Road & Head On \\
\hline Avg. Obs. Crashes/Year/Site & 1.1312 & 0.2458 \\
MAD/Year/Site & 0.1784 & 0.0528 \\
MPE/Year/Site & 0.0033 & 0.0011 \\
\hline Crash Type & Side Swipe (OD) & \\
\hline Avg. Obs. Crashes/Year/Site & 0.1621 & \\
MAD/Year/Site & 0.0369 & \\
MPE/Year/Site & 0.0009 & \\
\hline
\end{tabular}
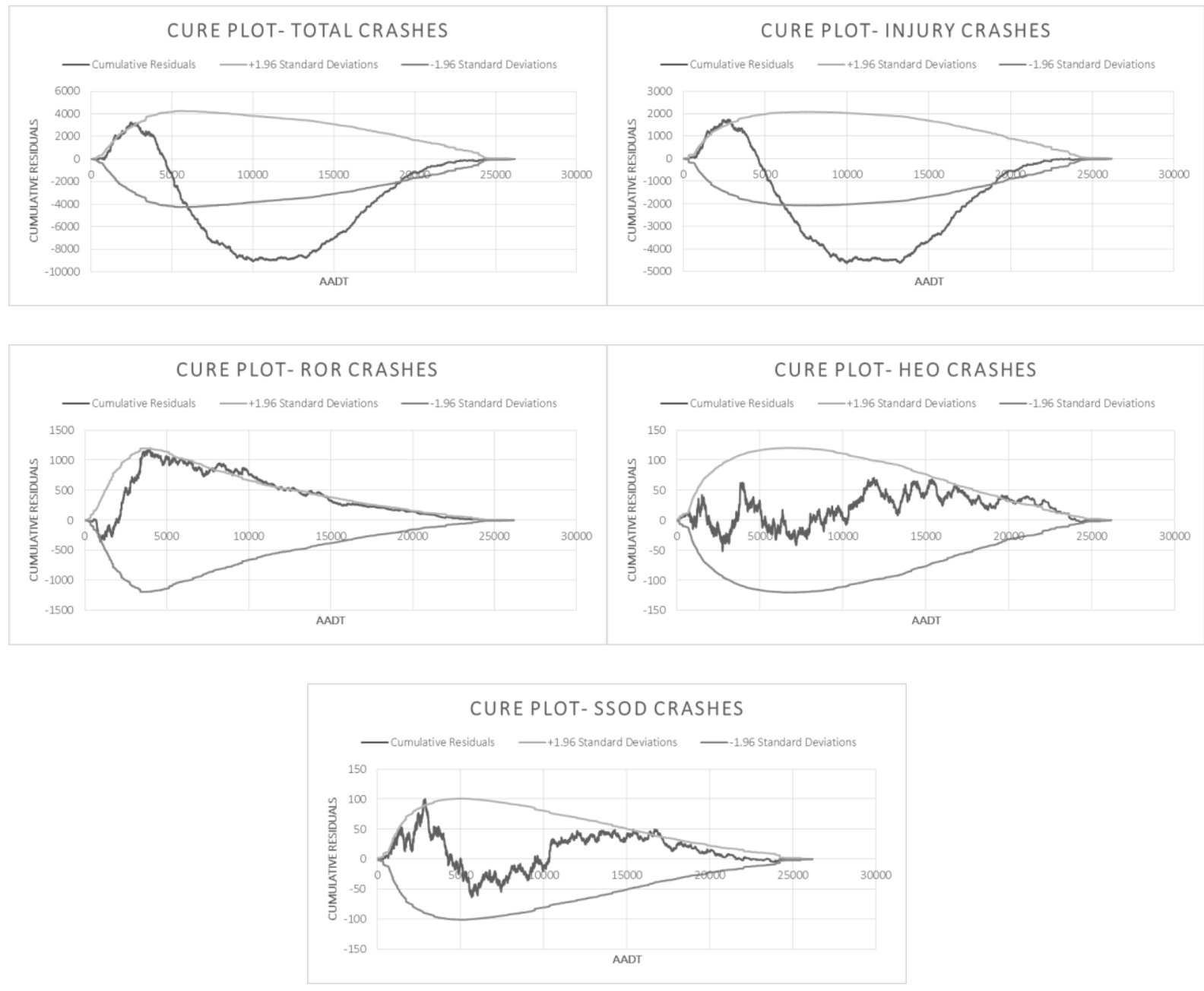

Figure 5.5 CURE Plots for Combined Model A 
The CURE plots (Figure 5.5) for the combined Kentucky + Pennsylvania models show a similar trend to the CURE plots for models developed using individual state data as shown in Figures 5.3 and 5.4. For the range of AADT between $\sim 6000$ and $\sim 19000$, the cumulative residuals lie outside the $95 \%$ confidence boundaries for total, injury and run off road crashes suggesting of a bias in the predictions in this AADT range. This can once again be indicative of long periods of over/under prediction by these models (Hauer, 2014). The CURE plots for head on and sideswipe opposite direction crashes do oscillate consistently and stay well within the $95 \%$ confidence boundaries suggesting of a better fit. However, as noted earlier, CURE plots alone cannot be used to judge the significance of the models and since the other goodness of prediction measures do suggest of a good overall fit, we can say that overall these models can predict crashes reasonably well.

\subsection{Modelling the Interaction of Combination Rumble Strips with Shoulder Width - Model B}

The model form used for developing the models incorporating the interaction between the presence of combination rumble strips and the shoulder width was as follows:

Crashes $/$ Km $/$ Year $=e^{\alpha+\beta_{3}} \times A A D T^{\beta_{1}} \times e^{\beta_{2} \times(\text { ShoulderWidth } \times R S)}$

Where;

$A A D T=$ Average annual daily traffic,

Shoulder Width = Shoulder width in meters, and

$R S=$ Presence of center line plus shoulder rumble strips: 1 if present, 0 if not present. 
Tables 5.15 and 5.16 show the coefficient estimates, over dispersion parameters $(K)$ and the goodness of prediction measures for models for various crash severities. CURE plots for all models are shown in Figure 5.6.Table 5.15 Estimates and Dispersion Parameters for

\section{Combined Model B}

\begin{tabular}{|c|c|c|c|c|}
\hline \multirow{2}{*}{$\begin{array}{l}\text { Crash Type } \\
\text { Coefficient }\end{array}$} & \multicolumn{2}{|l|}{ Total } & \multicolumn{2}{|l|}{ Injury } \\
\hline & $\begin{array}{c}\text { Estimate } \\
\text { (Standard Error) }\end{array}$ & Pr>ChiSq & $\begin{array}{c}\text { Estimate } \\
\text { (Standard Error) }\end{array}$ & Pr>ChiSq \\
\hline$\alpha$ & $-6.2437(0.0381)$ & $<0.0001$ & $-7.0470(0.0435)$ & $<0.0001$ \\
\hline 81 & $0.7266(0.0046)$ & $<0.0001$ & $0.7437(0.0052)$ & $<0.0001$ \\
\hline 82 & $-0.1538(0.0301)$ & $<0.0001$ & $-0.1365(0.0360)$ & 0.0001 \\
\hline B3 (Kentucky) & $0.3118(0.0270)$ & $<0.0001$ & $-0.2412(0.0314)$ & $<0.0001$ \\
\hline B3 (Pennsylvania) & 0 & - & 0 & - \\
\hline$K$ & \multicolumn{2}{|l|}{0.4550} & \multicolumn{2}{|l|}{0.4446} \\
\hline Crash Type & \multicolumn{2}{|c|}{ Run Off Road } & \multicolumn{2}{|c|}{ Head On } \\
\hline Coefficient & $\begin{array}{c}\text { Estimate } \\
\text { (Standard Error) }\end{array}$ & Pr>ChiSq & $\begin{array}{c}\text { Estimate } \\
\text { (Standard Error) }\end{array}$ & Pr>ChiSq \\
\hline$\alpha$ & $-5.9688(0.0677)$ & $<0.0001$ & $-10.7378(0.1158)$ & $<0.0001$ \\
\hline 81 & $0.4902(0.0083)$ & $<0.0001$ & $0.8818(0.0135)$ & $<0.0001$ \\
\hline 82 & $-0.3078(0.0605)$ & $<0.0001$ & $-0.2230(0.0863)$ & 0.0098 \\
\hline B3 (Kentucky) & $0.8301(0.0401)$ & $<0.0001$ & $-0.1442(0.0629)$ & 0.0219 \\
\hline 83 (Pennsylvania) & 0 & - & 0 & - \\
\hline$K$ & \multicolumn{2}{|l|}{0.9604} & \multicolumn{2}{|l|}{0.7295} \\
\hline Crash Type & \multicolumn{2}{|c|}{ Side Swipe (OD) } & & \\
\hline Coefficient & $\begin{array}{c}\text { Estimate } \\
\text { (Standard Error) }\end{array}$ & Pr>ChiSq & & \\
\hline$\alpha$ & $-10.3251(0.1385)$ & $<0.0001$ & & \\
\hline 81 & $0.7741(0.0162)$ & $<0.0001$ & & \\
\hline 82 & $-0.2592(0.0868)$ & 0.0028 & & \\
\hline 83 (Kentucky) & $0.9595(0.0573)$ & $<0.0001$ & & \\
\hline 83 (Pennsylvania) & 0 & - & & \\
\hline$K$ & 0.8040 & & & \\
\hline
\end{tabular}

As can be seen from Tables 5.15 and 5.16, the estimates of $\beta_{1}, \beta_{2}$ and $\beta_{3}$ are highly significant $(\mathrm{P}<0.01)$ for all the models. The goodness of prediction measures also suggests of reasonably good fits in that the MAD/year/site (Mean Absolute Deviation) and the MPE/year/site (Mean Prediction Error) for all the models are small (close to zero in all cases) when compared to the average observed crashes per year per site. For example, for total crashes the MAD/year/site is 0.616 and MPE/year/site is 0.011 compared to an average of $\sim 5.86$ total crashes per year per site. 
Table 5.16 Goodness of Prediction Measures for Combined Model B

\begin{tabular}{|c|c|c|}
\hline \multicolumn{2}{|c|}{ Crash Type } & Total \\
\hline Avg. Obs. Crashes/Year/Site & 5.8576 & 2.9772 \\
MAD/Year/Site & 0.6161 & 0.3471 \\
MPE/Year/Site & 0.0111 & 0.0061 \\
\hline Crash Type & Run Off Road & Head On \\
\hline Avg. Obs. Crashes/Year/Site & 1.1312 & 0.2458 \\
MAD/Year/Site & 0.1783 & 0.0528 \\
MPE/Year/Site & 0.0033 & 0.0011 \\
\hline Crash Type & Side Swipe (OD) & \\
\hline Avg. Obs. Crashes/Year/Site & 0.1621 & \\
MAD/Year/Site & 0.0369 & \\
MPE/Year/Site & 0.0009 & \\
\hline
\end{tabular}
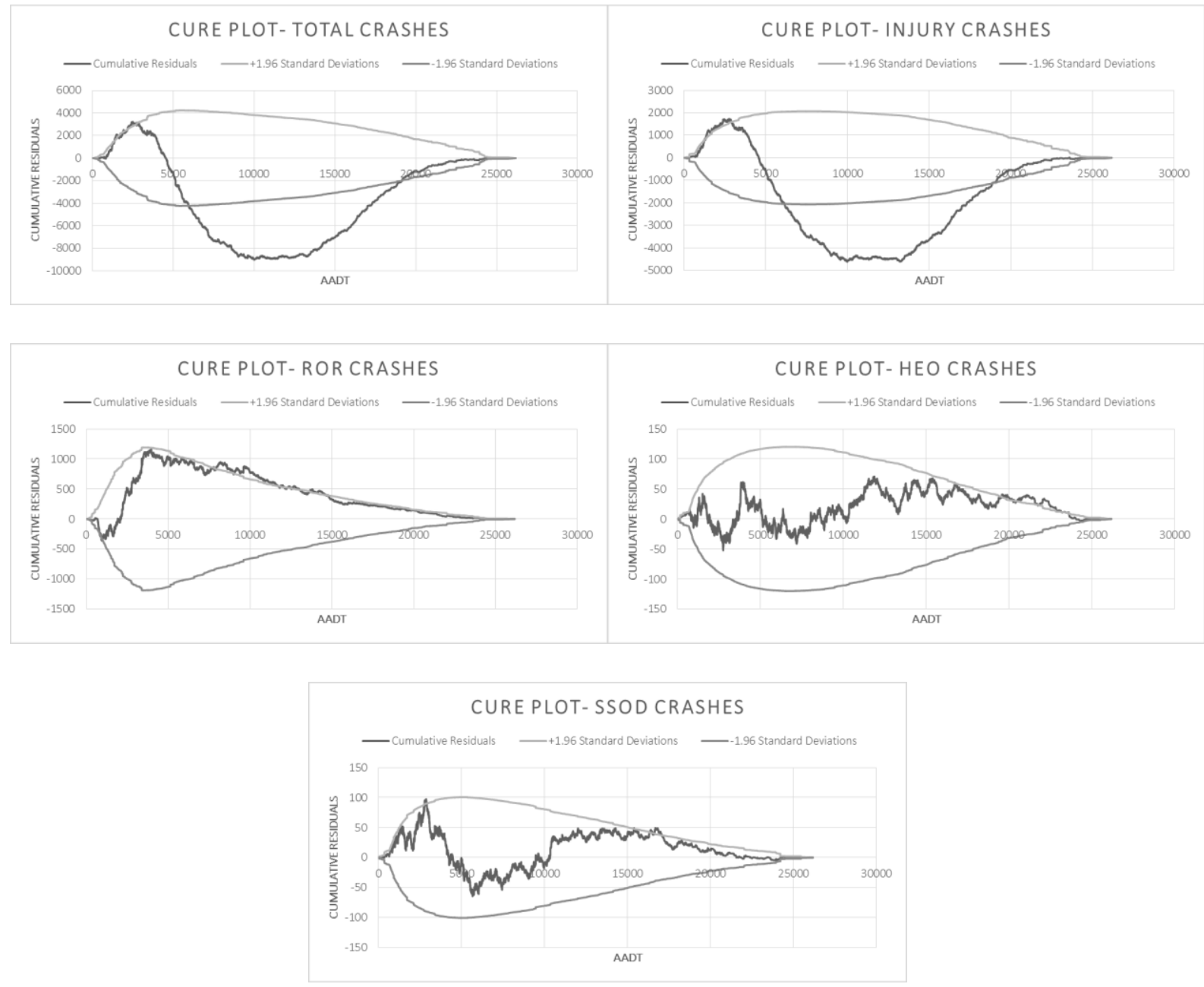

Figure 5.6 CURE Plots for Combined Model B 
The CURE plots (Figure 5.6) show a similar trend to the CURE plots for Model A shown in Figure 5.5 in that for the range of AADT between $\sim 6000$ and $\sim 19000$, the cumulative residuals lie outside the $95 \%$ confidence boundaries for total, injury and run off road crashes suggesting of a bias in the predictions in this AADT range. This can once again be indicative of long periods of over/under prediction by these models (Hauer, 2014). The CURE plots for head on and sideswipe opposite direction (SSOD) crashes do oscillate consistently and stay well within the $95 \%$ confidence boundaries suggesting of a better fit. However, as mentioned before, CURE plots alone cannot be used to judge the significance of the models and since the other goodness of prediction measures do suggest of a good overall fit, we can say that overall these models can predict crashes reasonably well.

\subsubsection{Application of Crash Prediction Models for Estimating Crash Modification Factors for Combination Rumble Strips}

The models shown in Equation 5-1 to 5-6 could be used to derive crash modification factors.

- Model A (for Kentucky, Pennsylvania and Combined data) would yield a single value CMF.

- Model B (for Kentucky, Pennsylvania and Combined data) would yield a crash modification function.

The crash modification factor derived from Model A would take the following form:

$C M F=\exp ^{\beta_{x} \times R S}$

Where; 
$C M F=$ Crash Modification Factor,

$\beta_{x}=\beta_{2}$ for all models (see Tables 5.5, 5.9 and 5.13), and

$R S=$ Presence of center line plus shoulder rumble strips: 1 if present, 0 if not present.

Table 5.17 below shows the CMF estimates from Equation 5-7 and compares them to the CMF estimates from the empirical Bayes study done by Persaud et al. (2016) with the dataset used in this section. Statistical significance of the differences in the CMF estimates was also tested at the 5\% level of significance (using the two-tailed t-test) and can be seen in Table 5.17. Figure 5.7 shows the comparison between the CMFs in the form of a bar graph for easy visual understanding.

Table 5.17 Comparison of CMF Estimates with CMFs from Persaud et al. (2016)

\begin{tabular}{|c|c|c|c|}
\hline State & Crash Type & CMF (SE) & $\begin{array}{c}\text { CMF from Persaud et al. } \\
2016 \text { (SE) }\end{array}$ \\
\hline \multirow{5}{*}{ Kentucky } & \multirow{5}{*}{$\begin{array}{c}\text { Total } \\
\text { Injury } \\
\text { Run Off Road } \\
\text { Head On } \\
\text { Side Swipe (OD) }\end{array}$} & $0.753(0.202)$ & $0.842(0.054)$ \\
\hline & & $0.714(0.210)$ & $0.812(0.088)$ \\
\hline & & $0.541(0.245)$ & $0.613(0.073)$ \\
\hline & & $0.444(0.357)$ & $0.480(0.142)$ \\
\hline & & $0.721(0.308)$ & $0.891(0.210)$ \\
\hline \multirow{5}{*}{ Pennsylvania } & \multirow{5}{*}{$\begin{array}{c}\text { Total } \\
\text { Injury } \\
\text { Run Off Road } \\
\text { Head On } \\
\text { Side Swipe (OD) }\end{array}$} & $0.806(0.055)$ & $0.975(0.046)$ \\
\hline & & $0.889(0.067)$ & $1.019(0.063)$ \\
\hline & & $0.756(0.117)$ & $0.920(0.103)$ \\
\hline & & $0.844(0.107)$ & $1.021(0.210)$ \\
\hline & & $0.786(0.122)$ & $0.907(0.246)$ \\
\hline \multirow{5}{*}{$\begin{array}{c}\text { Combined } \\
\text { (Kentucky }+ \\
\text { Pennsylvania) }\end{array}$} & \multirow{5}{*}{$\begin{array}{c}\text { Total } \\
\text { Injury } \\
\text { Run Off Road } \\
\text { Head On } \\
\text { Side Swipe (OD) }\end{array}$} & $0.790(0.027)$ & $0.800(0.025)$ \\
\hline & & $0.844(0.031)$ & $0.771(0.034)$ \\
\hline & & $0.704(0.040)$ & $0.742(0.041)$ \\
\hline & & $0.665(0.063)$ & $0.632(0.085)$ \\
\hline & & $0.662(0.057)$ & $0.767(0.097)$ \\
\hline
\end{tabular}

Note: Differences between CMFs (for the Crash Type) Statistically Insignificant at 5\% Level shown in boldface

As can be seen from the results in Table 5.17, the cross-sectional models developed in this section estimate slightly larger crash reductions compared to the results from Persaud et al. 
(2016). For example, for Kentucky, the model estimated a 25\% reduction in crashes (with a standard error of 0.202 ) compared to $16 \%$ by Persaud et al. (2016) (with a standard error of 0.054). The differences in CMF estimates from Kentucky data for all crash types are insignificant at the 5\% level showing that the two estimates are comparable statistically.

For Pennsylvania, the differences in CMF estimates for total and injury crashes are significant at the 5\% level, although, interestingly the cross-sectional analysis suggested that there is a benefit, in contrast to the before-after analysis. The CMFs for the other crash types are statistically comparable. For the models using combined dataset, it can be seen that the CMFs are closer to the CMFs estimated by Persaud et al. (2016) and have low standard errors. The differences in CMF estimates from the combined data are insignificant at the 5\% level for all crash types (except for injury crashes) showing that once again the two estimates are, in general, statistically comparable.

Overall, the results from the cross-sectional analysis seem to follow a similar trend to the results from the empirical Bayes study and the CMF estimates are comparable to those from the empirical Bayes study. A similar trend in the CMFs can also be seen from the bar graphs (Figure 5.7). 

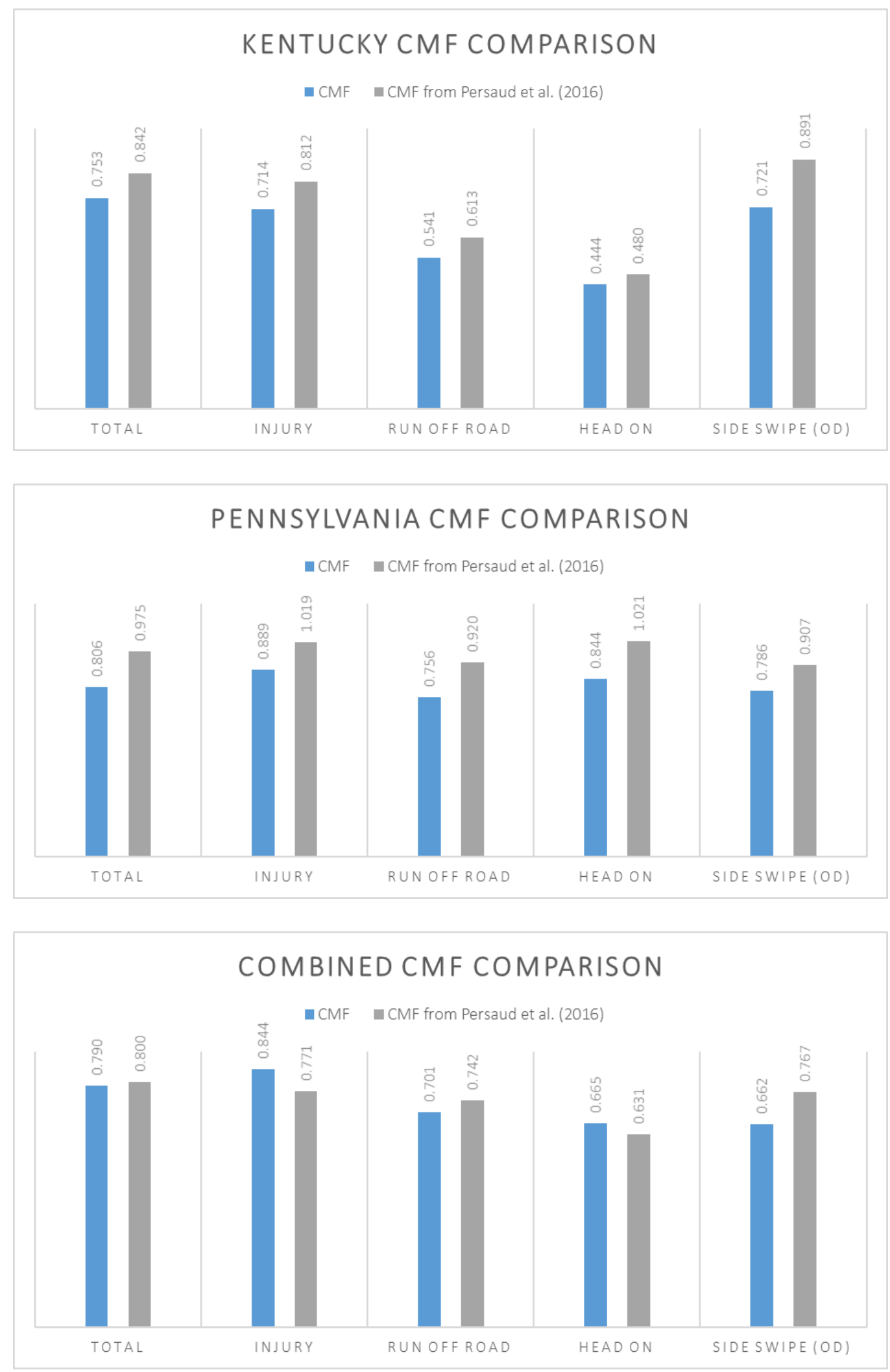

Figure 5.7 Bar Graphs for the Comparison of Rumble Strips CMFs 
Model B presents the interaction between the shoulder width and the presence of combination rumble strips. The crash modification function derived from Model B would take the following form:

$C M F=e^{\beta_{x} \times(\text { Shoulder Width } \times R S)}$

Where;

$C M F=$ Crash Modification Factor,

$\beta_{x}=\beta_{2}$ for all models (see Tables 5.7, 5.11 and 5.15),

Shoulder Width $=$ Shoulder width in meters, and

$R S=$ Presence of center line plus shoulder rumble strips: 1 if present, 0 if not present.

Figures $5.8-5.10$ below plot the effect on the crash reduction of varying shoulder width assuming that the combination rumble strips are present. For the plots, the base case is assumed to be shoulder width of 0 , which would translate to a CMF of 1 . CMFs for increasing shoulder width were found by comparing their safety to the safety at shoulder width of 0 . For example, a CMF of 0.5 would suggest a $50 \%$ crash reduction compared to shoulder width of 0 under similar circumstances. The plots are shown for shoulder widths up to $2 \mathrm{~m}$ as shoulder widths above $2 \mathrm{~m}$ are very rare in reality.

It can be seen from the plots that the safety performance of the combination rumble strips increases with an increase in the shoulder width. For example, for a shoulder width of $2 \mathrm{~m}$, the CMF for total crashes that would materialize in Kentucky would be $\sim 0.82$ (18\% reduction in crashes) and $\sim 0.77$ ( $23 \%$ reduction in crashes) in Pennsylvania. 


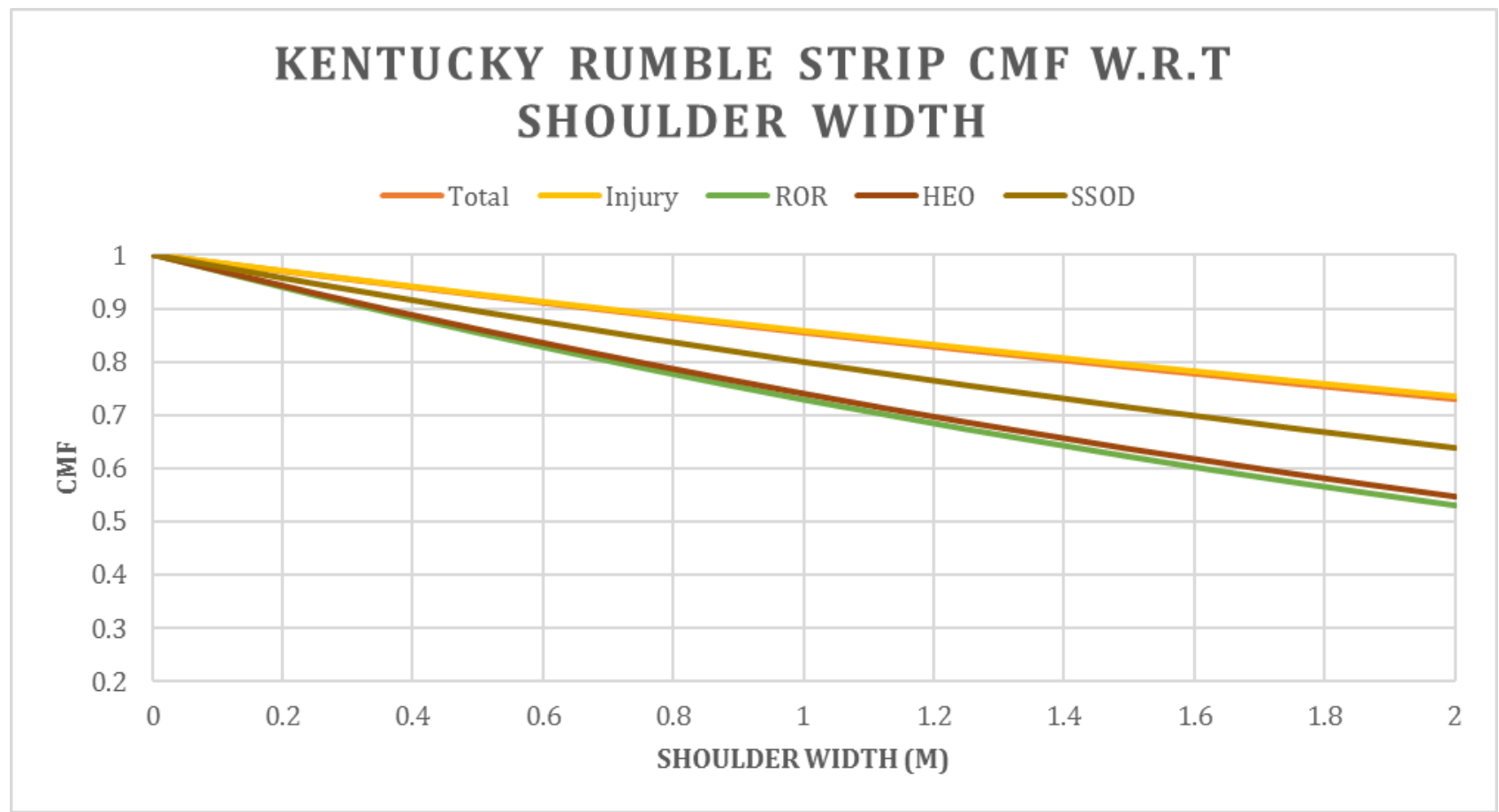

Figure 5.8 Comparison of CMFs for Varying Shoulder Width (Kentucky)

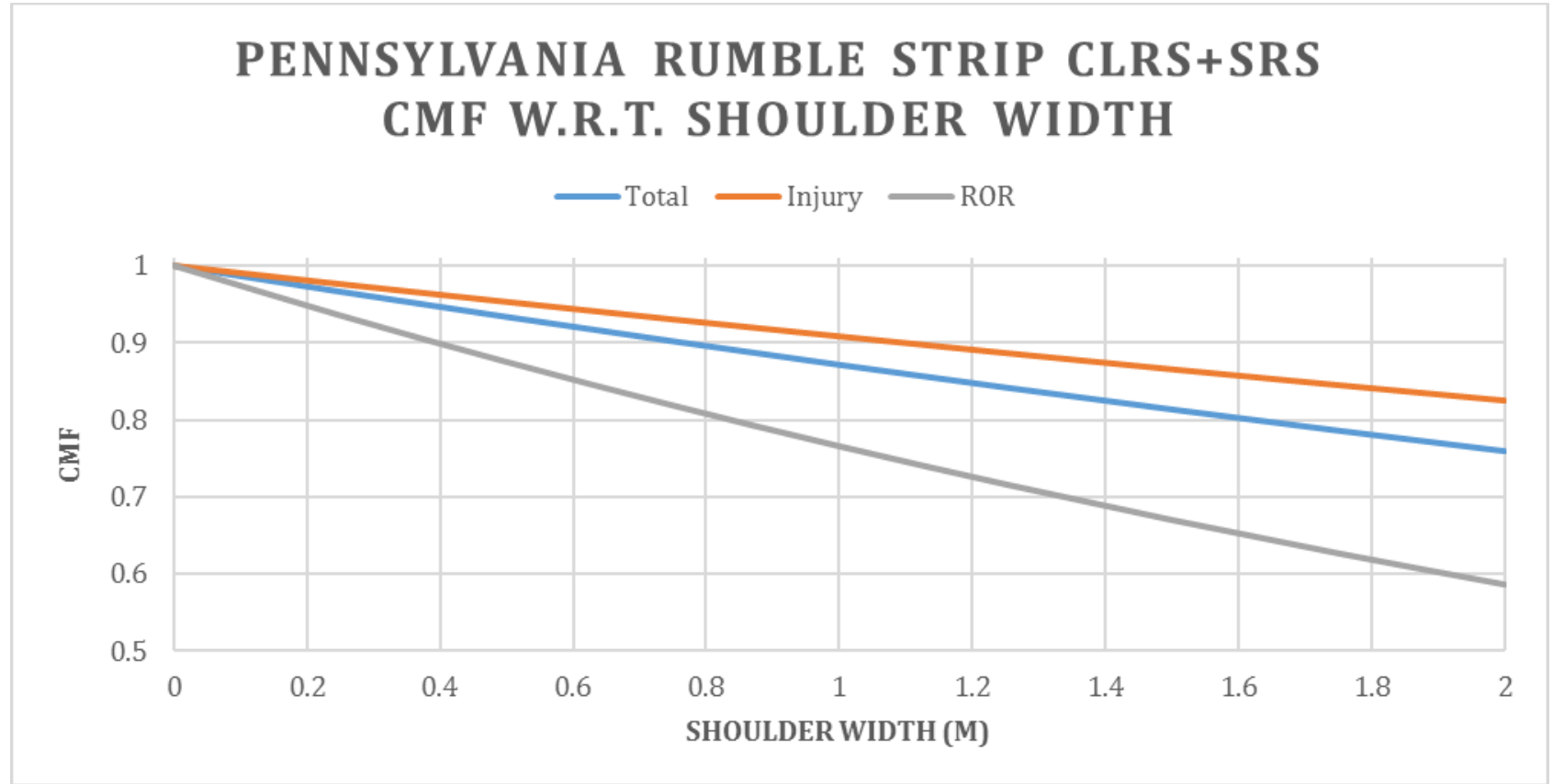

Figure 5.9 Comparison of CMFs for Varying Shoulder Width (Pennsylvania) 


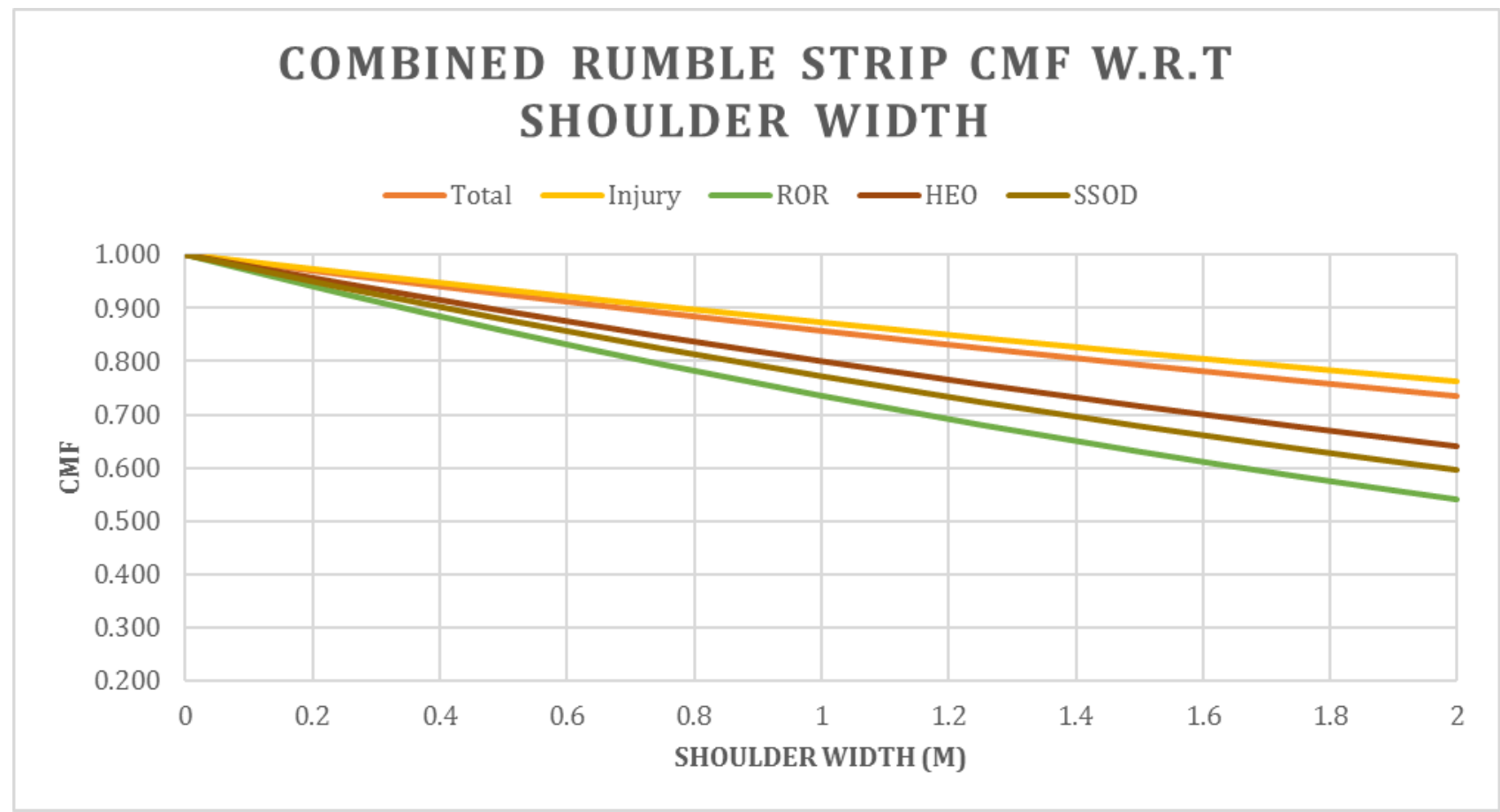

Figure 5.10 Comparison of CMFs for Varying Shoulder Width (Combined Data)

The plots also show that the reduction in crashes at higher shoulder widths are not due to the confounding effects of low speeds since the effect of high shoulder widths on safety was similar in both Kentucky (where all the segments had the same speed limit) and Pennsylvania (where the speed limits were within a $35 \mathrm{~km} / \mathrm{hr}$ range)

\subsubsection{Summary of the Combination Rumble Strip Case Study}

This section presented first of the three case studies used to evaluate the consistency of crash modification factors estimated from cross-sectional analysis using before-after data compared to CMFs estimated from empirical Bayes before-after evaluation. Crash prediction models were developed incorporating the presence of combination rumble strips and the interaction between shoulder widths and the presence of combination rumble strips. The model parameters 
were statistically significant to the 5\% level and had low standard errors. The CURE plots for total, injury and run off road crashes showed over/under prediction by the models but overall other goodness of prediction measures suggested of a good overall fit.

To further demonstrate the capabilities of this approach, estimates of CMFs for presence of combination rumble strips were compared to the empirical Bayes evaluation conducted by Persaud et al. (2016). The differences in the CMF estimates (compared to Persaud et al.) were mostly insignificant at the 5\% level, showing that the two estimates are statistically comparable in general. The results also indicated that the safety effects of changing shoulder width in the presence of combination rumble strips to increase as the shoulder width increases irrespective of the speed limit. Notably, this relationship could not be established in the before-after analysis by Persaud et al. (2016) who concluded that "the analysis found no clear trend between the CMF and shoulder width".

\subsection{Safety Evaluation of Wet Reflective Pavement Markings}

This section continues the focus on evaluating the consistency in the estimation of CMFs from cross-sectional analysis using actual before/after databases. It presents the second case study in this investigation using wet reflective pavement markings as a design element and data from a before-after study by Lyon et al. (2016). 


\subsubsection{Background/Motivation}

Wet reflective pavements markings are designed to provide an improved level of retro reflectivity during wet road surface conditions (Lyon et al., 2016). They can be applied on the road surface in various different forms including paint, tape, or thermoplastic materials.

According to Lyon et al. (2016), research assessing the safety effects of wet reflective pavement markings has been rare and limited in scope at the time of their study. Most of the studies available focus on the general relationship between retro reflectivity and crashes. As a result, the Highway Safety Manual (AASTHO, 2010) does not provide a CMF for the effect of wet reflective pavement markings.

A study done by Smadi et al. (2008) analyzed the relationship between three years of pavement marking retro reflectivity data collected by Iowa Department of Transportation and nighttime run off road and cross centre line crashes on rural two-lane roadways. They used logistic regression to assess the increased probability of target crashes when retro reflectivity values are lower. They did not a correlation between poor pavement marking retro reflectivity and higher crash probability. However, at lower values of retro reflectivity, they did find a weak but statistically significant relationship between crashes and retro reflectivity. Another study by Bahar et al. (2006) developed models for retro reflectivity of pavement markings based on the color, age, marker type, climate region and amount of snow removal to evaluate safety impact of retro reflectivity on nighttime non intersection in California. Their results indicated no relationship between nighttime no intersection crashes and retro reflectivity. Similar to the study conducted by Bahar et al. (2006), Donnell et al. (2009) in their study performed an exploratory analysis to determine the existence of statistical association between pavement 
marking retro reflectivity and traffic crash frequency. They developed models of pavement marking retro reflectivity degradation for selected highways in North Carolina using artificial neural networks, and subsequently, combined the estimates of pavement marking retro reflectivity levels with roadway and crash frequency data. They then estimated the monthly target crash frequencies using generalized estimating equations. Target crashes in their study included night time crashes linked visibility, dry roadway, run off road and sideswipe crashes. They found that on two-lane roads the regression parameter estimated for yellow and white pavement markings were negative, showing a decrease in crashes with increased retro reflectivity levels. For multi-lane roads they found lower crashes with increased retro reflectivity of white pavement markings, and vice versa for yellow pavement markings. Similarly, Carlson et al. (2015) in their study developed the relationship between retro reflectivity and crash data from North Carolina to demonstrate the effects of pavement marking retro reflectivity to nighttime crashes on rural two-lane roads. They found a decrease in nighttime crashes of between $15 \%$ - 30\% with increased level of pavement marking retro reflectivity.

The main objective of this section is to explore the use of cross-sectional regression to develop a crash modification function (CMFunction) to estimate CMFs for application of wet reflective pavement markings. For this purpose, crash prediction models were developed using using negative binomial regression and a combination of the reference site data and the after period data at the treatment sites to derive functions that can subsequently be used to estimate CMFs. These CMFs were then compared to recent study by Lyon et al. (2016), where wet reflective 
pavement markings were evaluated using empirical Bayes before/after analysis with the datasets used for the cross-sectional regression.

\subsubsection{Summary of Data}

The data for this chapter were provided by North Carolina and Wisconsin Department's of Transportation and consisted of freeways and multi-lane divided roads, respectively. The data consisted of both the locations and dates of the installation of wet reflective pavement markings alongside information on road geometry, volumes and crashes for both reference and installation sites. Tables 5.18 and 5.19 show some summary statistics and crash data for the North Carolina freeway sites. The data consisted of $372 \mathrm{~km}$ of treated sites and $153 \mathrm{~km}$ of reference sites with traffic and crash data ranging from 1998 - 2012. Tables 5.20 and 5.21 show some summary statistics and crash data for the Wisconsin multi-lane sites. The data consisted of $388 \mathrm{~km}$ of treated sites and $158 \mathrm{~km}$ of reference sites with traffic and crash data ranging from $2003-2012$. 
Table 5.18 Summary Statistics for North Carolina Freeway Data

\begin{tabular}{|c|c|c|c|}
\hline \multicolumn{4}{|c|}{ North Carolina Freeway Treatment Sites $(n=262)$} \\
\hline Variable & Minimum & Maximum & Average \\
\hline$A A D T$ & 10366.00 & 118760.00 & 45788.85 \\
\hline Length $(m)$ & 16.09 & 6448.63 & 586.03 \\
\hline Median $(m)$ & 0.00 & 33.53 & 8.98 \\
\hline Shoulder (m) & 0.00 & 5.33 & 2.78 \\
\hline Number of Lanes & 4.00 & 10.00 & 4.78 \\
\hline \multicolumn{4}{|c|}{ North Carolina Freeway Reference Sites $(n=790)$} \\
\hline Variable & Minimum & Maximum & Average \\
\hline$A A D T$ & 14005.00 & 159122.00 & 58669.06 \\
\hline Length $(m)$ & 16.09 & 6448.63 & 471.54 \\
\hline Median (m) & 0.00 & 33.53 & 7.50 \\
\hline Shoulder (m) & 0.76 & 5.02 & 2.66 \\
\hline Number of Lanes & 4.00 & 16.00 & 4.98 \\
\hline
\end{tabular}

Note: $1 \mathrm{mi}=1609.34 \mathrm{~m}, 1 \mathrm{ft}$. $=0.3048 \mathrm{~m}$

Table 5.19 Crash Statistics for North Carolina Freeway Data

\begin{tabular}{|c|c|c|c|}
\hline \multicolumn{4}{|c|}{ North Carolina Freeway Treatment Sites (n = 262) } \\
\hline Crash Type & Minimum & Maximum & Average \\
\hline Total & 0 & 125 & 9.86 \\
Injury & 0 & 36 & 2.41 \\
Run off Road & 0 & 11 & 0.51 \\
Side-Swipe Same-Direction & 0 & 22 & 1.49 \\
Wet Road & 0 & 34 & 2.03 \\
Night Time & 0 & 34 & 2.53 \\
\hline Night Time Wet Road & 0 & 13 & 0.64 \\
\hline North Carolina Freeway Reference Sites (n & $790)$ & \\
\hline Crash Type & Minimum & Maximum & Average \\
\hline Total & 0 & 713 & 39.59 \\
Injury & 0 & 236 & 12.51 \\
\hline Run off Road & 0 & 90 & 4.53 \\
\hline Side-Swipe Same-Direction & 0 & 129 & 5.49 \\
\hline Wet Road & 0 & 187 & 8.84 \\
Night Time & 0 & 137 & 10.37 \\
\hline Night Time Wet Road & 0 & 42 & 2.57 \\
\hline
\end{tabular}


Table 5.20 Summary Statistics for Wisconsin Multi Lane Data

\begin{tabular}{|c|c|c|c|}
\hline \multicolumn{4}{|c|}{ Wisconsin Multi-Lane Divided Treatment Sites ( $\mathbf{n}=408$ ) } \\
\hline & Minimum & Maximum & Average \\
\hline Variable & 2880.00 & 56640.00 & 16118.77 \\
\hline AADT & 16.09 & 2961.19 & 952.51 \\
Length $(m)$ & 0.00 & 115.82 & 10.65 \\
Median $(m)$ & 0.00 & 5.49 & 1.98 \\
Shoulder $(m)$ & 2.00 & 6.00 & 4.18 \\
\hline Number of Lanes & Minimum & Maximum & Average \\
\hline Wisconsin Multi-Lane Divided Reference Sites (n= 150) & 8835.82 \\
\hline Variable & 1813.00 & 29391.00 & 1051.22 \\
\hline AADT & 16.09 & 3234.77 & 4.41 \\
\hline Length $(m)$ & 0.00 & 36.57 & 2.09 \\
\hline Median $(m)$ & 0.00 & 3.96 & 4.16 \\
\hline Shoulder $(m)$ & 2.00 & 6.00 & \\
\hline Number of Lanes & & & \\
\hline
\end{tabular}

Note: $1 \mathrm{mi}=1609.34 \mathrm{~m}, 1 \mathrm{ft} .=0.3048 \mathrm{~m}$

Table 5.21 Crash Statistics for Wisconsin Multi Lane Data

\begin{tabular}{|c|c|c|c|}
\hline \multicolumn{4}{|c|}{ Wisconsin Multi-Lane Divided Treatment Sites (n = 408) } \\
\hline Crash Type & Minimum & Maximum & Average \\
\hline Total & 0 & 31 & 1.13 \\
Injury & 0 & 12 & 0.37 \\
Run off Road & 0 & 4 & 0.14 \\
Side-Swipe Same-Direction & 0 & 6 & 0.21 \\
Wet Road & 0 & 8 & 0.17 \\
Night Time & 0 & 4 & 0.23 \\
Night Time Wet Road & 0 & 3 & 0.04 \\
\hline Wisconsin Multi-Lane Divided Reference Sites (n=150) \\
\hline Crash Type & Minimum & Maximum & Average \\
\hline Total & 0 & 43 & 3.95 \\
Injury & 0 & 21 & 1.68 \\
\hline Run off Road & 0 & 6 & 0.58 \\
\hline Side-Swipe Same-Direction & 0 & 8 & 0.55 \\
Wet Road & 0 & 10 & 0.75 \\
Night Time & 0 & 8 & 0.87 \\
\hline Night Time Wet Road & 0 & 3 & 0.22 \\
\hline
\end{tabular}




\subsubsection{Model Fitting and Evaluation}

Consistent with state-of-the-art methods, generalized linear modelling, with the specification of a negative binomial (NB) error structure (as discussed in Section 3.4), was used to develop the crash prediction models (Persaud et al., 2012) using the SAS software (SAS, 2016).

\subsubsection{Model Fitting Using North Carolina Data}

Two models were developed using the North Carolina freeway data. The first form modelled the presence of the wet reflective pavement markings yielding a crash modification factor for the presence of the markings. The second form looked at the interaction between the presence of the wet reflective pavement markings and the median width. This would lead to a crash modification function where the magnitude of crash reduction will not only be based on the presence of the markings, but will also depend on the median width.

\subsection{Modeling the Presence of Wet Reflective Pavement Markings - Model $\boldsymbol{A}$}

The model form used for developing the models incorporating the presence of wet reflective pavement markings were as follows:

Crashes $/ \mathrm{Km} /$ Year $=e^{\alpha} \times A A D T^{\beta_{1}} \times e^{\beta_{2} \times \text { Lanes }} \times e^{\beta_{3} \times \text { Median Width }} \times e^{\beta_{4} \times W R P M}$

Where;

$A A D T=$ Average annual daily traffic,

Lanes $=1$ if 4 Lanes, 2 if 6 Lanes and 3 if $>6$ Lanes, 
Median Width = Median width in meters, and

$W R P M=$ Presence of wet reflective pavement markings: 1 if present, 0 if not present.

Shoulder width was not included in the model since it did not yield a significant estimate.

Tables 5.22 and 5.23 show the coefficient estimates, over dispersion parameters $(\mathrm{K})$ and the goodness of prediction measures for models for various crash severities. The CURE plots for all models are shown in Figure 5.11.

Table 5.22 Estimates and Dispersion Parameters for North Carolina Model A

\begin{tabular}{|c|c|c|c|c|c|c|c|c|}
\hline Crash Type & \multicolumn{2}{|l|}{ Total } & \multicolumn{2}{|l|}{ Injury } & \multicolumn{2}{|c|}{ Run Off Road } & \multicolumn{2}{|c|}{ Side Swipe (SD) } \\
\hline Coefficient & $\begin{array}{c}\text { Estimate } \\
\text { (Standard Error) }\end{array}$ & Pr>Chisq & $\begin{array}{c}\text { Estimate } \\
\text { (Standard Error) }\end{array}$ & Pr>Chisq & $\begin{array}{c}\text { Estimate } \\
\text { (Standard Error) }\end{array}$ & Pr>Chisq & $\begin{array}{c}\text { Estimate } \\
\text { (Standard Error) }\end{array}$ & Pr>Chisq \\
\hline$\alpha$ & $-15.0978(0.8644)$ & $<0.0001$ & $-16.6150(0.8855)$ & $<0.0001$ & $-17.5600(1.1035)$ & $<0.0001$ & $-21.1730(0.9828)$ & $<0.0001$ \\
\hline 81 & $0.6658(0.0856)$ & $<0.0001$ & $1.6894(0.0873)$ & $<0.0001$ & $1.6758(0.1084)$ & $<0.0001$ & $2.0361(0.0968)$ & $<0.0001$ \\
\hline 82 & $-0.2919(0.0933)$ & $<0.0001$ & $-0.3797(0.0889)$ & $<0.0001$ & $-0.2898(0.1014)$ & $<0.0001$ & $-0.2733(0.0907)$ & $<0.0001$ \\
\hline 83 & $-0.0429(0.0102)$ & $<0.0001$ & $-0.0308(0.0096)$ & 0.0014 & $-0.0133(0.0109)$ & 0.1650 & $-0.0596(0.0108)$ & $<0.0001$ \\
\hline 84 & $-0.0699(0.0169)$ & 0.0496 & $-0.1725(0.0830)$ & $<0.0001$ & $-0.1241(0.0268)$ & $<0.0001$ & $-0.0819(0.0299)$ & 0.1285 \\
\hline$K$ & & & 1.5550 & & 1.6726 & & 1.3564 & \\
\hline Crash Type & \multicolumn{2}{|c|}{ Wet Road } & \multicolumn{2}{|c|}{ Night Time } & \multicolumn{2}{|c|}{ Wet Road-Night Time } & & \\
\hline Coefficient & $\begin{array}{c}\text { Estimate } \\
\text { (Standard Error) }\end{array}$ & Pr>Chisq & $\begin{array}{c}\text { Estimate } \\
\text { (Standard Error) }\end{array}$ & Pr>ChiSq & $\begin{array}{c}\text { Estimate } \\
\text { (Standard Error) }\end{array}$ & Pr $>$ Chisq & & \\
\hline$\alpha$ & $-12.8494(0.9355)$ & $<0.0001$ & $-14.5658(0.9032)$ & $<0.0001$ & $-15.3138(1.0927)$ & $<0.0001$ & & \\
\hline 81 & $1.2914(0.0917)$ & $<0.0001$ & $1.4874(0.0891)$ & $<0.0001$ & $1.4232(0.1077)$ & $<0.0001$ & & \\
\hline 82 & $-0.2306(0.0998)$ & 0.0208 & $-0.1729(0.0915)$ & $<0.0001$ & $-0.3480(0.1095)$ & 0.0015 & & \\
\hline 63 & $-0.0292(0.0119)$ & 0.0141 & $-0.0396(0.0102)$ & 0.0001 & $-0.0392(0.0123)$ & 0.0014 & & \\
\hline 84 & $-0.1958(0.1154)$ & 0.0260 & $-0.1821(0.0723)$ & 0.0211 & $-0.2330(0.0931)$ & 0.0047 & & \\
\hline$K$ & 1.9089 & & 1.5996 & & 1.5734 & & & \\
\hline
\end{tabular}

Table 5.23 Goodness of Prediction Measures for North Carolina Model A

\begin{tabular}{|c|c|c|c|c|}
\hline Crash Type & Total & Injury & Run Off Road & Side Swipe (SD) \\
\hline \multirow{3}{*}{$\begin{array}{c}\text { Avg. Obs. Crashes/Year/Site } \\
\text { MAD/Year/Site } \\
\text { MPE/Year/Site }\end{array}$} & 32.1844 & 10.0009 & 3.5304 & 4.5019 \\
\hline & 9.5787 & 2.9203 & 1.1283 & 1.5424 \\
\hline & 0.7826 & 0.2543 & 0.0991 & 0.1165 \\
\hline Crash Type & Wet Road & Night Time & Wet Road - Night & \\
\hline \multirow{3}{*}{$\begin{array}{c}\text { Avg. Obs. Crashes/Year/Site } \\
\text { MAD/Year/Site } \\
\text { MPE/Year/Site }\end{array}$} & 7.1501 & 8.4201 & 2.0903 & \\
\hline & 2.1170 & 2.3455 & 0.7130 & \\
\hline & 0.1751 & 0.1886 & 0.0604 & \\
\hline
\end{tabular}



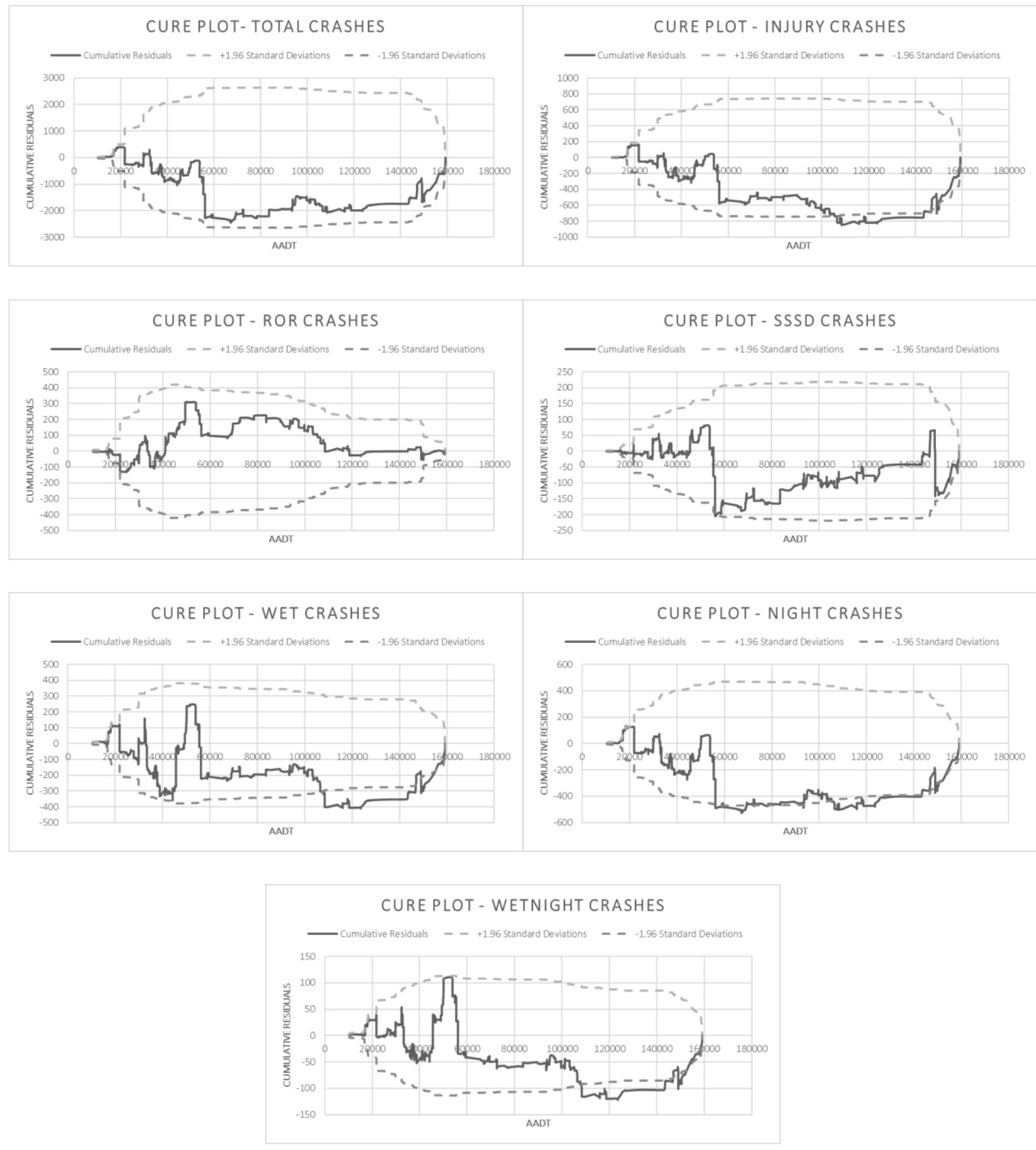

Figure 5.11 CURE Plots for North Carolina Model A

As can be seen from Tables 5.22 and 5.23, almost all of the coefficient estimates are highly significant $(\mathrm{P}<0.05)$ for all the models. There are few parameters (for example, median width in the run off road model) that are significant at the $15 \%$ level, which, combined with the high 
significance of other parameters, indicate significant overall results. The goodness of prediction measures also suggests of reasonably good fits in that the MAD/year/site (Mean Absolute Deviation) and the MPE/year/site (Mean Prediction Error) for all the models are small when compared to the average observed crashes per year per site. Even though the MAD/year/site values seem to higher in almost all cases, these values are still small when compared to the average observed crashes per year per site. For example, for total crashes the MAD/year/site is 9.578 and MPE/year/site is 0.782 compared to an average of $\sim 32.18$ total crashes per year per site.

The CURE plots for all models (Figure 5.11) show that the cumulative residuals lie mostly between the $95 \%$ confidence boundaries and oscillate consistently. In all cases, it can be seen that there a few steep vertical jumps and drops which are indicative of presence of possible outliers in the data (Hauer, 2014). CURE plots combined with other goodness of prediction measures suggest reasonably good predictions by all models.

\subsection{Modelling the Interaction of Wet Reflective Pavement Markings with Number of Lanes - Model B}

The model form used for developing the models incorporating the interaction of wet reflective pavement markings with number of lanes were as follows (interaction of wet reflective pavement markings with shoulder and median width were also explored, since these models provided inconsistent results they are not shown in this section):

Crashes $/$ Km $/$ Year $=e^{\alpha} \times A A D T^{\beta_{1}} \times e^{\beta_{2} \times \text { Median Width }} \times e^{\beta_{3} \times \text { Lanes } \times \text { WRPM }}$

Where; 
$A A D T=$ Average annual daily traffic,

Lanes $=1$ if 4 Lanes, 2 if 6 Lanes and 3 if $>6$ Lanes,

Median Width = Median width in meters, and

$W R P M=$ Presence of wet reflective pavement markings: 1 if present, 0 if not present.

Tables 5.24 and 5.25 show the coefficient estimates, over dispersion parameters $(\mathrm{K})$ and the goodness of prediction measures for models for various crash severities. The CURE plots for all the models are shown in Figure 5.12.

Table 5.24 Estimates and Dispersion Parameters for North Carolina Model B

\begin{tabular}{|c|c|c|c|c|c|c|c|c|}
\hline \multirow{2}{*}{$\begin{array}{l}\text { Crash Type } \\
\text { Coefficient }\end{array}$} & \multicolumn{2}{|l|}{ Total } & \multicolumn{2}{|l|}{ Injury } & \multicolumn{2}{|c|}{ Run Off Road } & \multicolumn{2}{|c|}{ Side Swipe (SD) } \\
\hline & $\begin{array}{c}\text { Estimate } \\
\text { (Standard Error) }\end{array}$ & Pr>ChiSq & $\begin{array}{c}\text { Estimate } \\
\text { (Standard Error) }\end{array}$ & Pr>ChiSq & $\begin{array}{c}\text { Estimate } \\
\text { (Standard Error) }\end{array}$ & Pr>ChiSq & $\begin{array}{c}\text { Estimate } \\
\text { (Standard Error) }\end{array}$ & Pr>Chisq \\
\hline$\alpha$ & $-13.4488(0.7547)$ & $<0.0001$ & $-15.3623(0.7730)$ & $<0.0001$ & $-16.2388(0.9681)$ & $<0.0001$ & $-19.4547(0.8553)$ & $<0.0001$ \\
\hline 81 & $1.4574(0.0680)$ & $<0.0001$ & $1.5184(0.0695)$ & $<0.0001$ & $1.4982(0.0875)$ & $<0.0001$ & $1.8212(0.0769)$ & $<0.0001$ \\
\hline 82 & $-0.0322(0.0103)$ & 0.0018 & $-0.0224(0.0095)$ & 0.0178 & $-0.0106(0.0016)$ & 0.0569 & $-0.0474(0.0105)$ & $<0.0001$ \\
\hline 83 & $-0.1182(0.0530)$ & 0.0022 & $-0.1679(0.0680)$ & $<0.0001$ & $-0.2905(0.0921)$ & $<0.0001$ & $-0.0746(0.0409)$ & 0.0059 \\
\hline K & \multicolumn{2}{|l|}{1.9454} & \multicolumn{2}{|l|}{1.5267} & \multicolumn{2}{|l|}{1.6369} & \multicolumn{2}{|l|}{1.3687} \\
\hline Crash Type & \multicolumn{2}{|c|}{ Wet Road } & \multicolumn{2}{|c|}{ Night Time } & \multicolumn{2}{|c|}{ Wet Road - Night Time } & & \\
\hline Coefficient & $\begin{array}{c}\text { Estimate } \\
\text { (Standard Error) }\end{array}$ & Pr>ChiSq & $\begin{array}{c}\text { Estimate } \\
\text { (Standard Error) }\end{array}$ & Pr>ChiSq & $\begin{array}{c}\text { Estimate } \\
\text { (Standard Error) }\end{array}$ & Pr>ChiSq & & \\
\hline$\alpha$ & $-12.1595(0.8368)$ & $<0.0001$ & $-13.0739(0.7848)$ & $<0.0001$ & $-13.9690(0.9402)$ & $<0.0001$ & & \\
\hline 81 & $1.1945(0.0750)$ & $<0.0001$ & $1.2958(0.0705)$ & $<0.0001$ & $1.2492(0.0842)$ & $<0.0001$ & & \\
\hline 82 & $-0.0216(0.0117)$ & 0.0643 & $-0.0308(0.0101)$ & 0.0024 & $-0.0335(0.0121)$ & 0.0056 & & \\
\hline 83 & $-0.1191(0.0408)$ & $<0.0001$ & $-0.1088(0.0218)$ & $<0.0001$ & $-0.1352(0.0248)$ & $<0.0001$ & & \\
\hline$K$ & 1.8628 & & 1.5862 & & 1.5349 & & & \\
\hline
\end{tabular}

Table 5.25 Goodness of Prediction Measures for North Carolina Model B

\begin{tabular}{|c|c|c|c|c|}
\hline Crash Type & Total & Injury & Run Off Road & Side Swipe (SD) \\
\hline \multirow{3}{*}{$\begin{array}{c}\text { Avg. Obs. Crashes/Year/Site } \\
\text { MAD/Year/Site } \\
\text { MPE/Year/Site }\end{array}$} & 32.1844 & 10.0009 & 3.5304 & 4.5019 \\
\hline & 9.5906 & 3.1587 & 1.1966 & 1.5116 \\
\hline & 0.8204 & 0.2813 & 0.1041 & 0.1209 \\
\hline Crash Type & Wet Road & Night Time & Wet Road - Night & \\
\hline \multirow{3}{*}{$\begin{array}{c}\text { Avg. Obs. Crashes/Year/Site } \\
\text { MAD/Year/Site } \\
\text { MPE/Year/Site }\end{array}$} & 7.1501 & 8.4201 & 2.0903 & \\
\hline & 2.1715 & 2.4459 & 0.7255 & \\
\hline & 0.1861 & 0.2040 & 0.0633 & \\
\hline
\end{tabular}



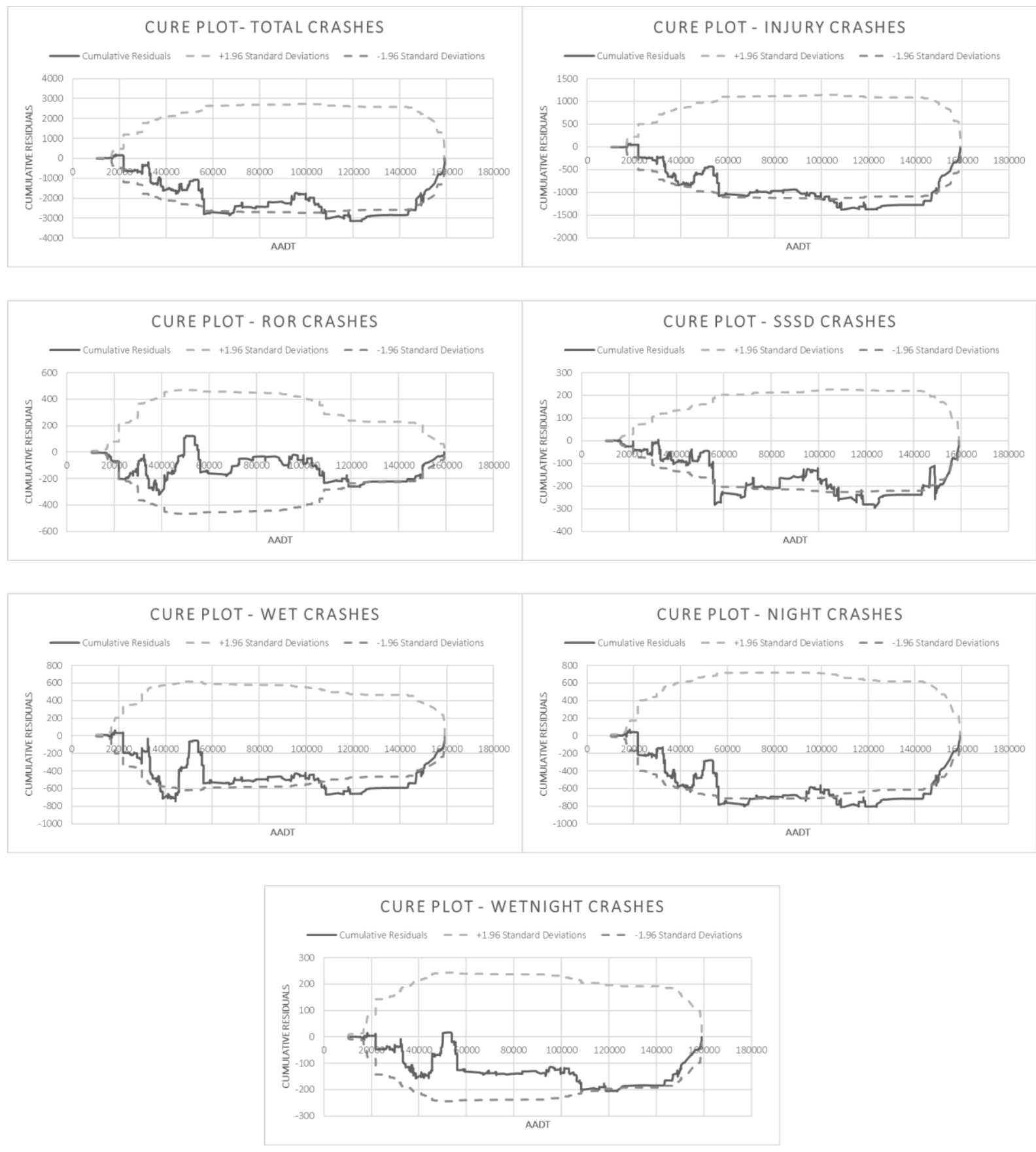

Figure 5.12 CURE Plots for North Carolina Model B

As can be seen from Tables 5.24 and 5.25, almost all of the coefficient estimates are highly significant $(\mathrm{P}<0.01)$ for all the models. The goodness of prediction measures also suggests of reasonably good fits in that the MAD/year/site (Mean Absolute Deviation) and the 
MPE/year/site (Mean Prediction Error) for all the models are small when compared to the average observed crashes per year per site. Even though the MAD/year/site values seem to higher in almost all cases, these values are still small when compared to the average observed crashes per year per site. For example, for total crashes the MAD/year/site is 9.591 and MPE/year/site is 0.820 compared to an average of $\sim 32.18$ total crashes per year per site. Similarly, for run off road crashes, the MAD/year/site is 1.196 and MPE/year/site is 0.104 compared to an average of $\sim 3.53$ run off road crashes per year per site.

The CURE plots for all models (Figure 5.12) show that the cumulative residuals oscillate consistently, but in all cases, tend to go outside the $95 \%$ confidence boundaries at higher values of AADT. Furthermore, it can be seen that all plots do show signs of steep vertical jumps and drops, indicating of possible outliers in the data (Hauer, 2014). However, CURE plot alone cannot be used to judge the significance of the models and since the other goodness of prediction measures do suggest of a good overall fit, we can say that overall these models can predict crashes reasonably well.

\subsubsection{Model Fitting Using Wisconsin Data}

In this part, multi lane divided road data from Wisconsin were used to develop the models. First, the presence of the wet reflective pavement markings was modelled yielding a crash modification factor for the presence of the markings. Other models were also explored to define the interaction between wet reflective pavement markings and various other variables including shoulder width, median width and number of lanes. None of these interactions came out to be significant and hence these models are not shown here. 
The model form used for developing the models incorporating the presence of wet reflective pavement markings were as follows:

Crashes $/$ Km $/$ Year $=e^{\alpha} \times A A D T^{\beta_{1}} \times e^{\beta_{2} \times \text { Shoulder Width }} \times e^{\beta_{3} \times W R P M}$

Where;

$A A D T=$ Average annual daily traffic,

Shoulder Width = Shoulder width in meters, and

$W R P M=$ Presence of wet reflective pavement markings: 1 if present, 0 if not present.

Median width and number of lanes were not included in the model as they did not yield a significant estimate. Tables 5.26 and 5.27 show the coefficient estimates, dispersion parameters and the goodness of prediction measures for models for various crash severities. The CURE plots for all models are shown in Figure 5.13.

Table 5.26 Estimates and Dispersion Parameters for Wisconsin Models

\begin{tabular}{|c|c|c|c|c|c|c|c|c|}
\hline \multirow{2}{*}{$\begin{array}{l}\text { Crash Type } \\
\text { Coefficient }\end{array}$} & \multicolumn{2}{|l|}{ Total } & \multicolumn{2}{|l|}{ Injury } & \multicolumn{2}{|c|}{ Run Off Road } & \multicolumn{2}{|c|}{ Side Swipe (SD) } \\
\hline & $\begin{array}{c}\text { Estimate } \\
\text { (Standard Error) }\end{array}$ & Pr>ChiSq & $\begin{array}{c}\text { Estimate } \\
\text { (Standard Error) }\end{array}$ & Pr>Chisq & $\begin{array}{c}\text { Estimate } \\
\text { (Standard Error) }\end{array}$ & Pr>Chisq & $\begin{array}{c}\text { Estimate } \\
\text { (Standard Error) }\end{array}$ & Pr>Chisc \\
\hline$\alpha$ & $-14.4712(1.2993)$ & $<0.0001$ & $-15.8067(1.8335)$ & $<0.0001$ & $-9.9158(1.7935)$ & $<0.0001$ & $-16.5213(2.2079)$ & $<0.0001$ \\
\hline 81 & $1.6692(0.1328)$ & $<0.0001$ & $1.7093(0.1867)$ & $<0.0001$ & $0.8437(0.1843)$ & $<0.0001$ & $1.6991(0.2257)$ & $<0.0001$ \\
\hline 82 & $-0.4807(0.0572)$ & $<0.0001$ & $-0.4331(0.0793)$ & $<0.0001$ & $-0.1496(0.0973)$ & 0.1244 & $-0.6385(0.0850)$ & $<0.0001$ \\
\hline 83 & $-0.3349(0.0474)$ & $<0.0001$ & $-0.7713(0.1054)$ & $<0.0001$ & $-1.0155(0.2261)$ & $<0.0001$ & $-0.1651(0.0446)$ & $<0.0001$ \\
\hline$K$ & \multicolumn{2}{|l|}{0.5771} & \multicolumn{2}{|l|}{0.7438} & \multicolumn{2}{|l|}{0.34} & & \\
\hline Crash Type & \multicolumn{2}{|c|}{ Wet Road } & \multicolumn{2}{|c|}{ Night Time } & \multicolumn{2}{|c|}{ Wet Road - Night Time } & & \\
\hline Coefficient & $\begin{array}{c}\text { Estimate } \\
\text { (Standard Error) }\end{array}$ & Pr>ChiSq & $\begin{array}{c}\text { Estimate } \\
\text { (Standard Error) }\end{array}$ & Pr>ChiSq & $\begin{array}{c}\text { Estimate } \\
\text { (Standard Error) }\end{array}$ & Pr>ChiSq & & \\
\hline$\alpha$ & $-17.7779(2.3568)$ & $<0.0001$ & $-13.6559(1.7318)$ & $<0.0001$ & $-15.7599(3.3208)$ & $<0.0001$ & & \\
\hline 81 & $1.8454(0.2405)$ & $<0.0001$ & $1.3862(0.1767)$ & $<0.0001$ & $1.4950(0.3388)$ & $<0.0001$ & & \\
\hline 82 & $-0.4838(0.0973)$ & $<0.0001$ & $-0.3092(0.0736)$ & $<0.0001$ & $-0.5088(0.1301)$ & $<0.0001$ & & \\
\hline 83 & $-0.5601(0.0684)$ & $<0.0001$ & $-0.6146(0.1003)$ & $<0.0001$ & $-0.6493(0.0879)$ & $<0.0001$ & & \\
\hline$K$ & \multicolumn{2}{|l|}{0.6341} & \multicolumn{2}{|l|}{0.2813} & \multicolumn{2}{|l|}{0.2494} & & \\
\hline
\end{tabular}


Table 5.27 Goodness of Prediction Measures for Wisconsin Models

\begin{tabular}{|c|c|c|c|c|}
\hline Crash Type & Total & Injury & Run Off Road & Side Swipe (SD) \\
\hline \multirow{3}{*}{$\begin{array}{c}\text { Avg. Obs. Crashes/Year/Site } \\
\text { MAD/Year/Site } \\
\text { MPE/Year/Site }\end{array}$} & 1.8870 & 0.7258 & 0.2634 & 0.3064 \\
\hline & 0.7655 & 0.3446 & 0.1441 & 0.1633 \\
\hline & 0.0832 & 0.0420 & 0.0143 & 0.0177 \\
\hline Crash Type & Wet Road & Night Time & Wet Road-Night & \\
\hline \multirow{3}{*}{$\begin{array}{c}\text { Avg. Obs. Crashes/Year/Site } \\
\text { MAD/Year/Site } \\
\text { MPE/Year/Site }\end{array}$} & 0.3279 & 0.4014 & 0.0896 & \\
\hline & 0.1751 & 0.2042 & 0.0501 & \\
\hline & 0.0221 & 0.0193 & 0.0080 & \\
\hline
\end{tabular}
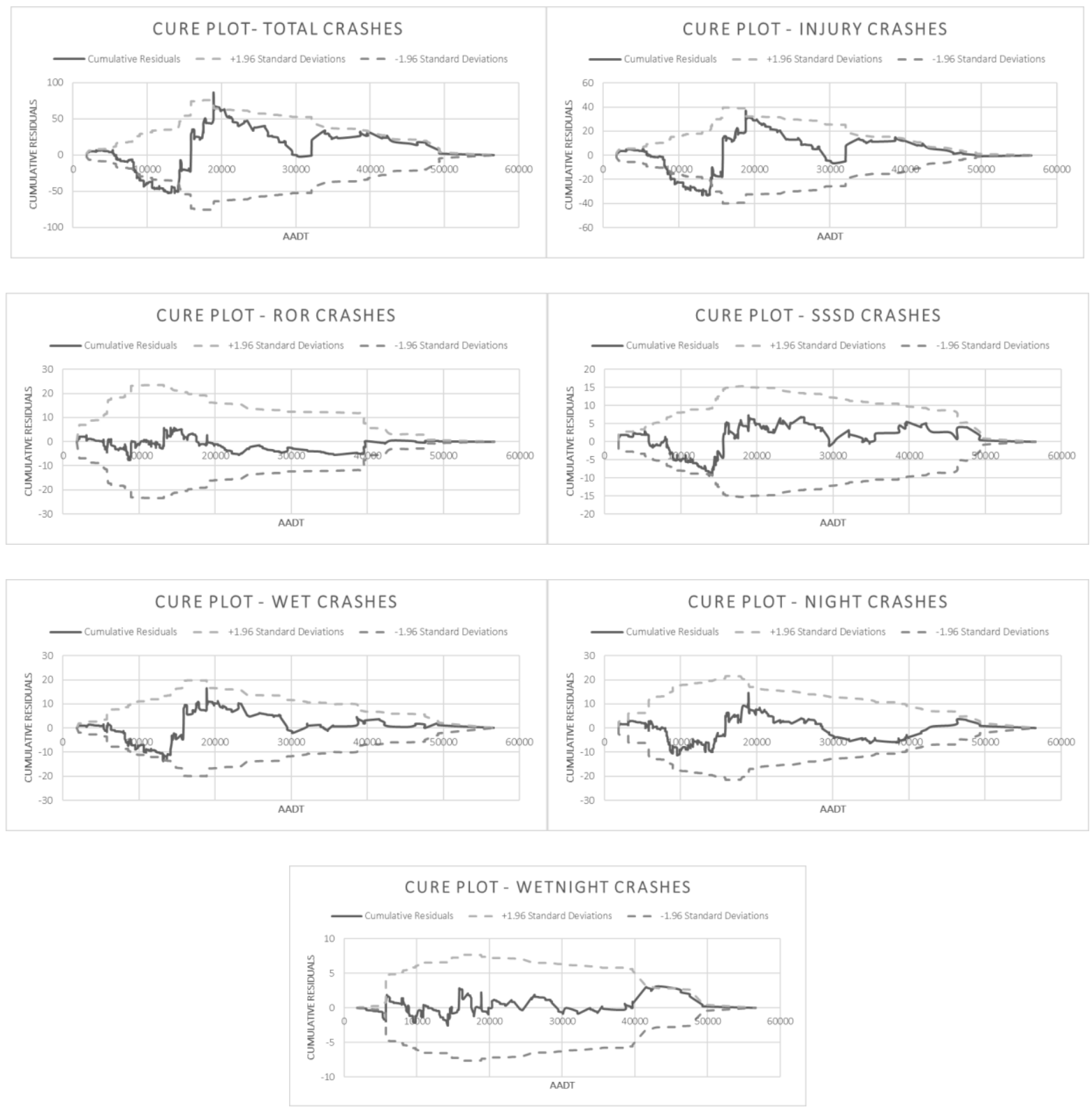

Figure 5.13 CURE Plots for Wisconsin Models 
As can be seen from Tables 5.26 and 5.27, all of the coefficient estimates are highly significant $(\mathrm{P}<0.01)$ for all the models. The goodness of prediction measures also suggests of reasonably good fits in that the MAD/year/site (Mean Absolute Deviation) and the MPE/year/site (Mean Prediction Error) for all the models are small when compared to the average observed crashes per year per site. Even though the MAD/year/site values seem to higher in almost all cases, these values are still small when compared to the average observed crashes per year per site. For example, for total crashes the MAD/year/site is 0.766 and MPE/year/site is 0.083 compared to an average of $\sim 1.89$ total crashes per year per site. Similarly, for run off road crashes, the MAD/year/site is 0.144 and MPE/year/site is 0.014 compared to an average of $\sim 0.26$ run off road crashes per year per site.

The CURE plots for all the models, shown in Figure 5.13, indicate that the cumulative residuals lie well within the $95 \%$ confidence boundaries and oscillate consistently for all the models, showing little or no bias. CURE plots combined with other goodness of prediction measures suggest that the models can predict crashes reasonably well.

\subsubsection{Application of Crash Prediction Models for Estimating Crash Modification Factors for Wet Reflective Pavement Markings}

The models shown in Equation 5-9 to 5-11, could be used to derive the crash modification factors.

- Equations 5-9 and 5-11 (for both North Carolina and Wisconsin) would yield a single value CMF.

- Equation 5-10 (for North Carolina) would yield a crash modification function. 
The crash modification factor derived from Equation 5-9 and 5-11 would take the following form:

$C M F=\exp ^{\beta_{x} \times W R P M}$

Where;

$\mathrm{CMF}=$ Crash Modification Factor,

$\beta_{x}=\beta_{4}$ for North Carolina model, $\beta_{3}$ for Wisconsin model (see Tables 5.22 and 5.26), and

$W R P M=$ Presence of wet reflective pavement markings: 1 if present, 0 if not present.

Tables 5.28 and 5.29 show the CMF estimates from Equation 5-12 for freeway (North Carolina) and multi-lane divided (Wisconsin) sites and compares them to the CMF estimates from the empirical Bayes study done by Lyon et al. (2016) using the dataset used in this chapter. Statistical significance of the differences in the CMF estimates was also tested at the 5\% level of significance (using the two-tailed t-test) and can be seen in Tables 5.28 and 5.29.

Table 5.28 Comparison of Freeway CMF Estimates with CMFs from Lyon et al. (2016)

\begin{tabular}{|c|c|c|}
\hline Crash Type & Freeway CMF (SE) & $\begin{array}{c}\text { Freeway CMF from Lyon } \\
\text { et al. } 2016 \text { (SE) }\end{array}$ \\
\hline Total & $0.932(0.017)$ & $0.977(0.020)$ \\
\hline Injury & $0.842(0.083)$ & $0.881(0.033)$ \\
\hline Run Off Road & $0.883(0.027)$ & $0.964(0.054)$ \\
\hline Side Swipe (SD) & $0.921(0.030)$ & $1.010(0.054)$ \\
\hline Wet Road & $0.822(0.115)$ & $0.861(0.040)$ \\
\hline Night Time & $0.934(0.072)$ & $0.966(0.038)$ \\
\hline Wet Road-Night & $0.792(0.093)$ & $0.979(0.080)$ \\
\hline
\end{tabular}

Note: Differences between CMFs (for the Crash Type) Statistically Insignificant at 5\% Level shown in boldface 
Table 5.29 Comparison of Multi-Lane Divided CMF Estimates with CMFs from Lyon et al. (2016)

\begin{tabular}{|c|c|c|}
\hline Crash Type & $\begin{array}{c}\text { Multi-Lane Divided CMF } \\
\text { (SE) }\end{array}$ & $\begin{array}{l}\text { Multi-Lane Divided CMF } \\
\text { from Lyon et al. } 2016\end{array}$ \\
\hline Total & $0.715(0.047)$ & $0.825(0.051)$ \\
\hline Injury & $0.462(0.105)$ & $0.595(0.059)$ \\
\hline Run Off Road & $0.362(0.226)$ & $0.538(0.078)$ \\
\hline Side Swipe (SD) & $0.848(0.045)$ & $0.941(0.115)$ \\
\hline Wet Road & $0.571(0.068)$ & $0.751(0.108)$ \\
\hline Night Time & $0.541(0.100)$ & $0.696(0.058)$ \\
\hline Wet Road-Night & $0.522(0.088)$ & $1.001(0.270)$ \\
\hline
\end{tabular}

Note: Differences between CMFs (for the Crash Type) Statistically Insignificant at 5\% Level shown in boldface

As can be seen from the results in Table 5.28, the cross-sectional models developed in this section estimate slightly larger crash reductions (with slightly large standard errors) for freeway segments compared to the results from Lyon et al. (2016). For example, the model estimated a $16 \%$ reduction in injury crashes (with a standard error of 0.017 ) compared to $12 \%$ by Lyon et al. (2016) (with a standard error of 0.033). Lyon et al. (2016) in their study do get an increase in side swipe same direction crashes of $0.1 \%$ (with a standard error of 0.054 ), which in fact is contrary to the crash counts of treatment and reference sites. The differences in CMF estimates (compared to Lyon et al.) are insignificant for injury, sideswipe same direction, wet road, and night time crashes showing the two estimates for these crashes are statistically comparable for these crash types. Although the differences between estimates for total, run off road, and wet road - night time crashes are significant at the 5\% level, interestingly the cross-sectional analysis suggested that there is a benefit, in contrast to the before-after analysis.

For multi-lane divided segments (Table 5.29), the CMFs again follow a similar trend. For example, the model predicts a $29 \%$ reduction in total crashes (with a standard error of 0.047 ) 
compared to $18 \%$ (with a standard error of 0.051 ) by Lyon et al. (2016). Lyon et al. (2016) in their study do predict wet road - night time crashes to stay unchanged i.e. a CMF of $\sim 1$, which in fact is contrary to the crash counts of treatment and reference sites. The differences in CMF estimates (compared to Lyon et al.) are insignificant for sideswipe same direction, wet road, and wet road - night time crashes showing the two estimates for these crashes are statistically comparable. Although the differences between estimates for total, injury, run off road, and night time crashes are significant at the 5\% level, interestingly the cross-sectional analysis suggested that there is a benefit, in contrast to the before-after analysis.

Overall, the results from the cross-sectional analysis seem to follow a similar trend to the results from the empirical Bayes study and the estimates are reasonably comparable to those from the empirical Bayes study. Figures 5.14 and 5.15 show the comparison between the CMFs in the form of a bar graph for easy visual understanding. The similar trend in the CMF estimates can be seen from the bar graphs.

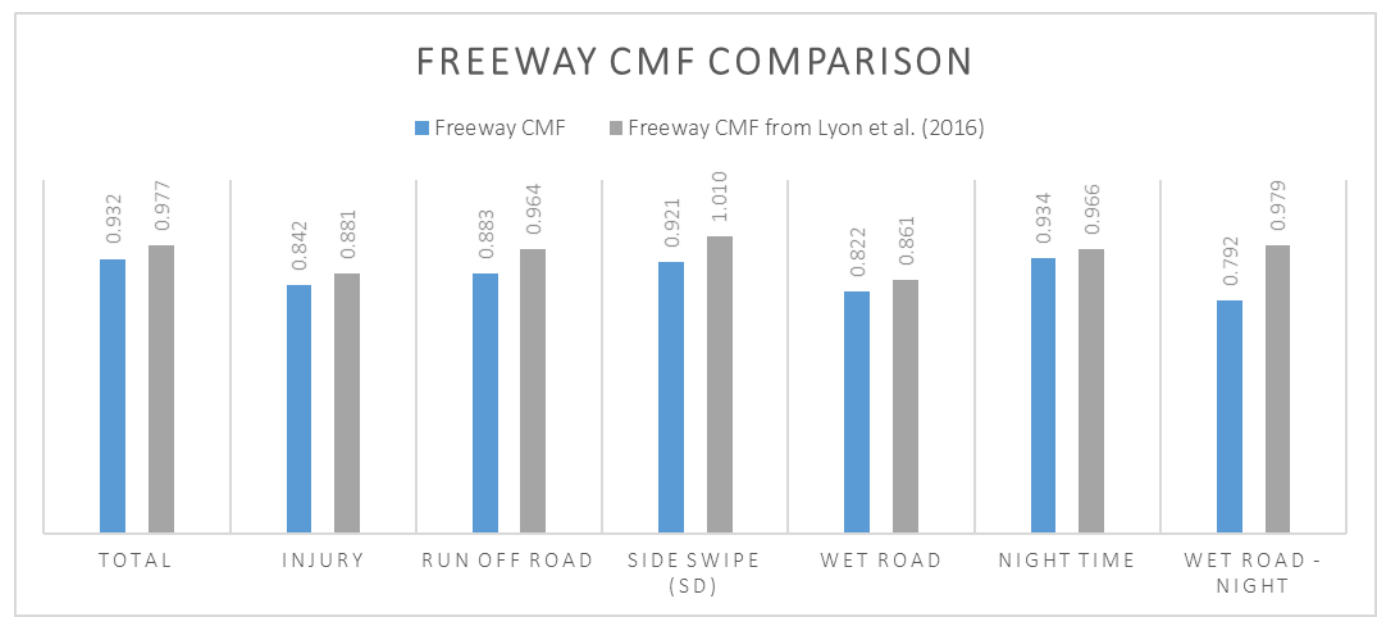

Figure 5.14 Bar Graph for the Comparison of Freeway CMFs 


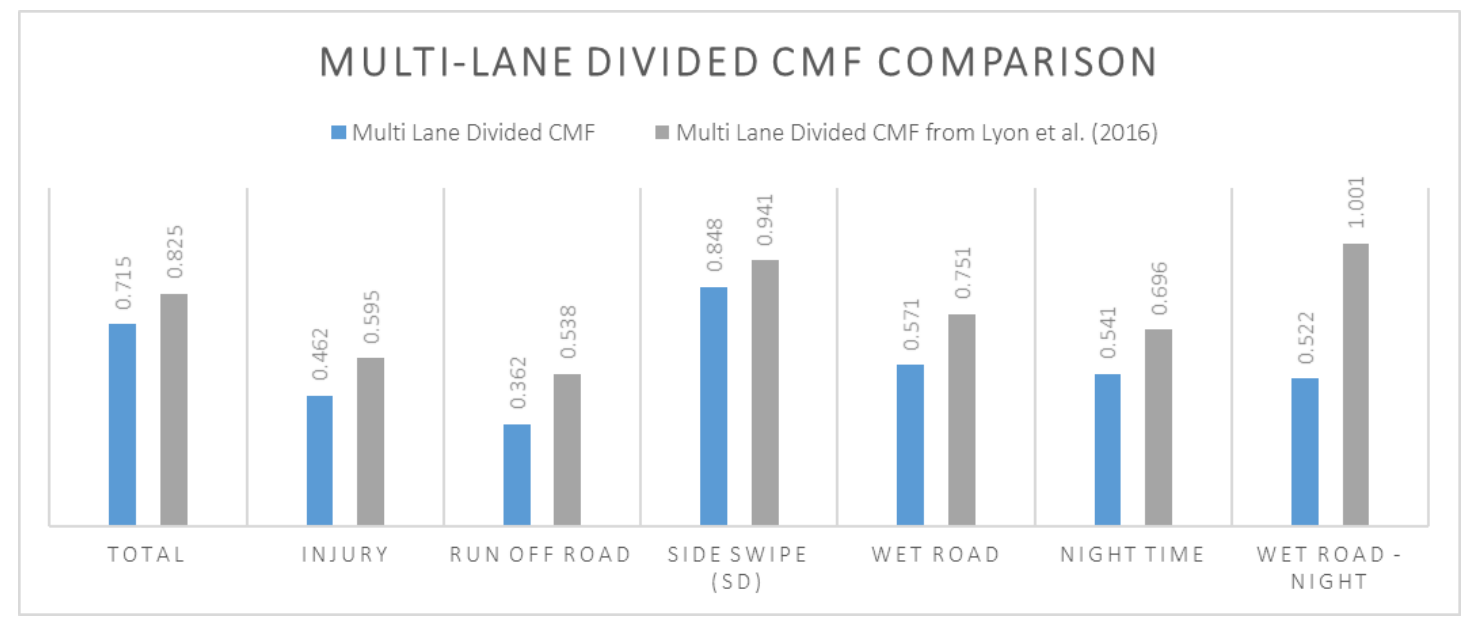

Figure 5.15 Bar Graph for the Comparison of Multi-Lane Divided CMFs

Equation 5-10 presents the interaction between the number of lanes and the presence of combination rumble strips. The crash modification function derived from Equation 5-10 would take the following form:

$C M F=e^{\beta_{x} \times(\text { Lanes } \times R S)}$

Where;

$\mathrm{CMF}=$ Crash Modification Factor,

$\beta_{x}=\beta_{3}$ from North Carolina freeway model (see Tables 5.24),

Lanes $=1$ if 4 Lanes, 2 if 6 Lanes and 3 if $>6$ Lanes, and

$W R P M=$ Presence of wet reflective pavement markings: 1 if present, 0 if not present.

Table 5.30 shows the effect on the crash reduction of varying number of lanes assuming that the wet reflective pavement markings are present. 


\begin{tabular}{|c|c|c|c|c|}
\hline Lanes & Total & Injury & $R O R$ & SSSD \\
\hline 4 Lanes & 0.889 & 0.845 & 0.748 & 0.928 \\
\hline 6 Lanes & 0.789 & 0.715 & 0.559 & 0.861 \\
\hline$>6$ Lanes & 0.701 & 0.604 & 0.418 & 0.799 \\
\hline Lanes & Wet & Night & Wet-Night & \\
\hline 4 Lanes & 0.888 & 0.897 & 0.874 & \\
\hline 6 Lanes & 0.788 & 0.804 & 0.763 & \\
\hline$>6$ Lanes & 0.700 & 0.722 & 0.667 & \\
\hline
\end{tabular}

Table 5.30 Comparison of Wet Reflective Pavement Marking CMFs for Varying Number of Lanes (North Carolina Freeways)

It can be seen from Table 5.30 that the safety performance of the wet reflective pavement markings increases with an increase in the number of lanes. For example, for a six- lane freeway, the CMF that would materialize for total crashes would be $\sim 0.79$ (21\% reduction in crashes) compared to a CMF of 0.89 for total crashes on four-lane freeways. CMFs for all crash types seem to follow a similar downward trend with run off road crashes seeing the biggest drop.

\subsubsection{Summary of the Wet-Reflective Pavement Marking Case Study}

This section presented second of the three case studies used to evaluate the consistency of crash modification factors estimated from cross-sectional analysis using before-after data compared to CMFs estimated from empirical Bayes before/after evaluation. Crash prediction models were developed incorporating the presence of wet reflective pavement markings and the interaction between median widths and the presence of wet reflective pavement markings. These models were statistically significant to the 5\% level and had low standard errors in almost all cases. The goodness of prediction measures also indicated a good fit. 
To further demonstrate the capabilities of this approach, estimates of CMFs for presence of wet reflective pavement markings were compared to the empirical Bayes evaluation conducted by Lyon et al. (2016). The comparison was generally favourable. The differences in the CMF estimates (compared to Lyon et al.) were mostly insignificant at the 5\% level, showing that the two estimates are statistically comparable in general. For cases, where the differences were significant at the 5\% level, interestingly the cross-sectional analysis suggested that there is a benefit, in contrast to the before-after analysis. The results also indicate that the safety effects of varying number of lanes in the presence of wet reflective pavement markings increased as the number of lanes increases. Notably, this relationship could not be established using the before-after analysis, with Lyon et al. (2016) concluding that "no differences or clear results were seen for number of lanes and the estimated CMFs".

\section{$\underline{5.3 \text { Safety Evaluation of Intersection Conflict Warning Systems }}$}

This section will continue the focus on evaluating the consistency in the estimation of CMFs from cross-sectional analysis using actual before/after databases. It presents the third and the final case study in this evaluation using intersection conflict warning systems as a design element and data from a before-after study by Himes et al. (2016).

\subsubsection{Background/Motivation}

Intersection conflict warning systems employ vehicle detectors to alert motorists of conflicting vehicles on an adjacent approach through the use of warning signs. It is a strategy used at intersections with limited sight distance and/or intersections with a history of crashes linked to

gap acceptance. Warning signs on major road approaches could read, "Vehicle Entering When 
Flashing", "Crossing Traffic When Flashing", or "Watch for Entering Traffic" (Himes et al., 2016). Warning signs on minor road approaches could read, "Traffic Approaching When Flashing" or "Look for Traffic" (Himes et al., 2016).

According to Himes e al. (2016), research assessing the safety effects of intersection conflict warning systems have been rare at the time of their study and cannot be considered to be definitive mostly because of the low sample sizes used. As a result, the Highway Safety Manual (AASTHO, 2010) does not provide a CMF for the effect of wet reflective pavement markings.

A study conducted by Bretherton and Miao (1999) used 18 sites with at least three preventable crashes in a year or at least one preventable crash for three consecutive years. These sites were installed with a post mounted sign with the messages, "Vehicle Approaching" and "Vehicle Entering Highway" on minor and major road approaches, respectively. Their results showed that the warning systems did effectively reduce the number of crashes linked to limited sight distance. Similarly, Hanscom (2001) conducted a before-after analysis for a collision countermeasure system at one site, Pennsylvania Department of Transportation (2007) conducted a before-after analysis of a post mounted at two locations, and Missouri Department of Transportation (2010) studied the safety effectiveness of post mounted warning systems at nine major and ten minor road approaches. Although all of these studies are relevant, their small sample sizes make it impossible to conduct a detailed safety review

The main objective of this section is to explore the development of crash prediction models to estimate CMFs for application of intersection conflict warning systems. Models were developed using using negative binomial regression and a combination of the reference site 
data and the after period data at the treatment sites to derive functions that can subsequently be used to estimate CMFs. These CMFs were then compared to recent study by Himes et al. (2016), where intersection conflict warning systems were evaluated using empirical Bayes before/after analysis with parts of the datasets used for the cross-sectional regression.

\subsubsection{Summary of Data}

The data for this chapter were provided by Minnesota, Missouri and North Carolina Department's of Transportation and consisted of four legged intersections with either two- or four-lanes on major road approaches. The data consisted of both the locations and dates of the installation of wet reflective pavement markings alongside information on road geometry, volumes and crashes for both reference and installation sites. Tables 5.31, 5.32 and 5.33 show some summary statistics and crash data Minnesota, Missouri and North Carolina. Crash Data was available ranging from $1992-2012$, but for the purpose of this evaluation only 7 years of data, 2006 - 2012, were used. Reference site data was used from 2006 - 2012. For treatment sites, only those that were treated in or after 2005 were taken into consideration. 
Table 5.31 Summary Statistics for Minnesota Data

\begin{tabular}{|c|c|c|c|}
\hline \multicolumn{4}{|c|}{ Minnessota Treatment Sites $(n=13)$} \\
\hline Data & Minimum & Maximum & Average \\
\hline Major AADT & 2033 & 21000 & 8415.24 \\
\hline Minor AADT & 310 & 4400 & 1392.34 \\
\hline Major Road Lanes & 2 & 4 & 3.11 \\
\hline Total Crashes/Year & 0 & 6 & 1.42 \\
\hline Injury Crashes/Year & 0 & 5 & 0.58 \\
\hline Right Angle Crashes/Year & 0 & 3 & 0.69 \\
\hline \multicolumn{4}{|c|}{ Minnessota Reference Sites $(n=63)$} \\
\hline Data & Minimum & Maximum & Average \\
\hline Major AADT & 900 & 18600 & 4349.76 \\
\hline Minor AADT & 224 & 2742 & 850.63 \\
\hline Major Road Lanes & 2 & 4 & 3.11 \\
\hline Total Crashes/Year & 0 & 9 & 1.71 \\
\hline Injury Crashes/Year & 0 & 5 & 0.69 \\
\hline Right Angle Crashes/Year & 0 & 4 & 1.04 \\
\hline
\end{tabular}

Table 5.32 Summary Statistics for Missouri Data

\begin{tabular}{|c|c|c|c|}
\hline \multicolumn{4}{|c|}{ Missouri Treatment Sites $(n=14)$} \\
\hline Data & Minimum & Maximum & Average \\
\hline Major AADT & 973 & 33685 & 10357.78 \\
\hline Minor AADT & 224 & 2742 & 850.63 \\
\hline Major Road Lanes & 2 & 4 & 3.11 \\
\hline Total Crashes/Year & 0 & 8 & 1.42 \\
\hline Injury Crashes/Year & 0 & 5 & 0.53 \\
\hline Right Angle Crashes/Year & 0 & 6 & 0.58 \\
\hline \multicolumn{4}{|c|}{ Missouri Reference Sites $(n=63)$} \\
\hline Data & Minimum & Maximum & Average \\
\hline Major AADT & 90 & 12770 & 4317.13 \\
\hline Minor AADT & 18 & 1412 & 398.97 \\
\hline Major Road Lanes & 2 & 4 & 2.88 \\
\hline Total Crashes/Year & 0 & 15 & 2.26 \\
\hline Injury Crashes/Year & 0 & 7 & 0.94 \\
\hline Right Angle Crashes/Year & 0 & 5 & 1.19 \\
\hline
\end{tabular}


Table 5.33 Summary Statistics for North Carolina Data

\begin{tabular}{|c|c|c|c|}
\hline \multicolumn{4}{|c|}{ North Carolina Treatment Sites $(n=56)$} \\
\hline Data & Minimum & Maximum & Average \\
\hline Major AADT & 830 & 31000 & 5517.17 \\
\hline Minor AADT & 370 & 4500 & 2022.32 \\
\hline Major Road Lanes & 2 & 4 & 2.36 \\
\hline Total Crashes/Year & 0 & 10 & 1.76 \\
\hline Injury Crashes/Year & 0 & 7 & 0.85 \\
\hline Right Angle Crashes/Year & 0 & 6 & 0.69 \\
\hline \multicolumn{4}{|c|}{ North Carolina Reference Sites $(n=62)$} \\
\hline Data & Minimum & Maximum & Average \\
\hline Major AADT & 1100 & 25000 & 9576.31 \\
\hline Minor AADT & 110 & 5600 & 1146.73 \\
\hline Major Road Lanes & 2 & 4 & 2.96 \\
\hline Total Crashes/Year & 0 & 10 & 2.58 \\
\hline Injury Crashes/Year & 0 & 7 & 1.32 \\
\hline Right Angle Crashes/Year & 0 & 6 & 1.56 \\
\hline
\end{tabular}

Because of the low number of treatment sites in Minnesota and Missouri, for the purpose of modelling, all the three datasets were combined to yield two sub sets of two-lane and four-lane intersections.

\subsubsection{Model Fitting and Evaluation}

Consistent with state-of-the-art methods, generalized linear modelling, with the specification of a negative binomial (NB) error structure (as discussed in Section 3.4), was used to develop the crash prediction models (Persaud et al., 2012) using the SAS software (SAS, 2016).

Models were developed using a combined dataset from all three states incorporating the presence of intersection conflict warning systems yielding a crash modification factor for the presence of intersection conflict warning systems. Other models were also explored to define the interaction between intersection conflict warning systems and various other variables including number of turn lanes, intersection angle, lighting at intersection, speed limit and 
median width on major route. None of these interactions came out to be significant and hence these models are not shown here. The only variable that would interact with the presence of intersection conflict warning systems would be the number of lanes on the approaches. Since the data were already disaggregated by the number of lanes, this interaction could not be explored.

\subsubsection{Model for Sites with Two Lanes on Major Road Approaches}

The model form used for developing the models incorporating the presence of intersection conflict warning systems was as follows:

Tables 5.34 and 5.35 show the coefficient estimates, over dispersion parameters $(\mathrm{K})$ and the goodness of prediction measures for models for various crash severities.

Table 5.34 Estimates and Dispersion Parameters for Two-Lane Model

\begin{tabular}{|c|c|c|c|c|}
\hline \multirow{2}{*}{$\begin{array}{l}\text { Crash Type } \\
\text { Coefficient }\end{array}$} & \multicolumn{2}{|l|}{ Total } & \multicolumn{2}{|l|}{ Injury } \\
\hline & $\begin{array}{c}\text { Estimate } \\
\text { (Standard Error) }\end{array}$ & Pr>ChiSq & $\begin{array}{c}\text { Estimate } \\
\text { (Standard Error) }\end{array}$ & Pr>Chisq \\
\hline$\alpha$ & $-5.0745(0.4795)$ & $<0.0001$ & $-6.9852(0.7153)$ & $<0.0001$ \\
\hline 81 & $0.6882(0.0554)$ & $<0.0001$ & $0.8301(0.0825)$ & $<0.0001$ \\
\hline 82 & $-0.3900(0.0618)$ & $<0.0001$ & $-0.5620(0.0859)$ & $<0.0001$ \\
\hline$K$ & \multicolumn{2}{|c|}{0.2531} & \multicolumn{2}{|l|}{0.3959} \\
\hline Crash Type & \multicolumn{2}{|c|}{ Right Angle } & & \\
\hline Coefficient & $\begin{array}{c}\text { Estimate } \\
\text { (Standard Error) }\end{array}$ & Pr>ChiSq & & \\
\hline$\alpha$ & $-4.9887(0.7250)$ & $<0.0001$ & & \\
\hline 81 & $0.6247(0.0839)$ & $<0.0001$ & & \\
\hline 82 & $-0.8820(0.0916)$ & $<0.0001$ & & \\
\hline$K$ & 0.6566 & & & \\
\hline
\end{tabular}


Table 5.35 Goodness of Prediction Measures for Two-Lane Model

\begin{tabular}{|c|c|c|}
\hline Crash Type & Total & Injury \\
\hline \multirow{3}{*}{$\begin{array}{c}\text { Avg. Obs. Crashes/Year/Site } \\
\text { MAD/Year/Site } \\
\text { MPE/Year/Site }\end{array}$} & 1.5196 & 0.7272 \\
\hline & 0.9130 & 0.4264 \\
\hline & 0.0465 & 0.0309 \\
\hline Crash Type & Right Angle & \\
\hline \multirow{3}{*}{$\begin{array}{c}\text { Avg. Obs. Crashes/Year/Site } \\
\text { MAD/Year/Site } \\
\text { MPE/Year/Site }\end{array}$} & 0.7608 & \\
\hline & 0.4867 & \\
\hline & 0.0343 & \\
\hline
\end{tabular}
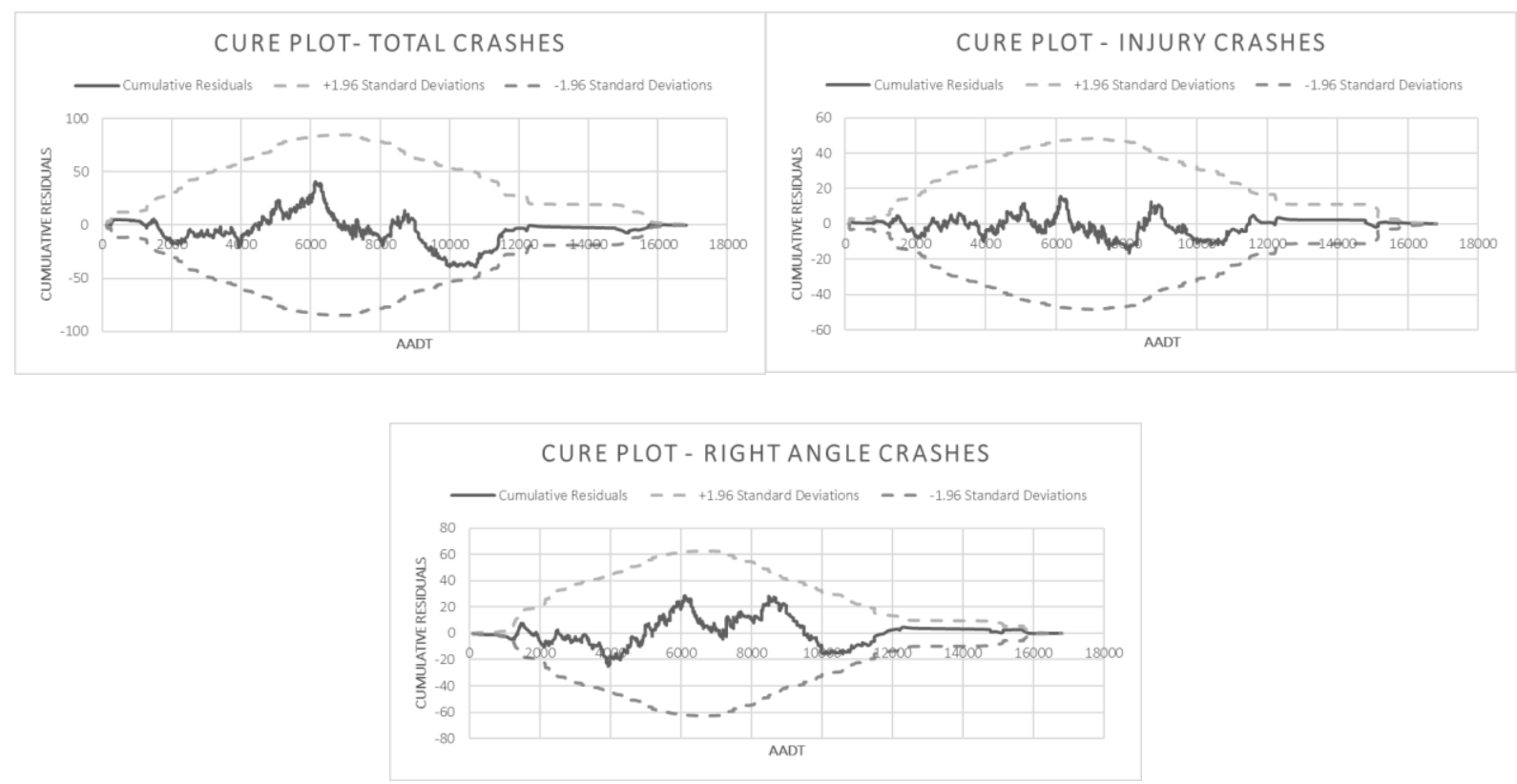

Figure 5.16 CURE Plots for Two-Lane Model

As can be seen from Tables 5.34 and 5.35, the estimates of $\beta_{1}$ and $\beta_{2}$ are highly significant $(\mathrm{P}<0.01)$ for all the models. The goodness of prediction measures also suggests of reasonably good fits in that the MAD/year/site (Mean Absolute Deviation) and the MPE/year/site (Mean Prediction Error) for all the models are small when compared to the average observed crashes per year per site. For example, for total crashes the MAD/year/site is 0.913 and MPE/year/site is 0.047 compared to an average of $\sim 1.52$ total crashes per year per site. 
The CURE plots for all models, shown in Figures 5.16, show that the cumulative residuals lie mostly between the 95\% confidence boundaries and oscillate consistently around the horizontal axis, showing little or no bias.

\subsubsection{Model for Sites with Four Lanes on Major Road Approaches}

The model form used for developing the models incorporating the presence of intersection conflict warning systems was as follows:

Crashes $/$ Year $=e^{\alpha} \times$ Entering $A A D T^{\beta_{1}} \times e^{\beta_{2} \times I C W S}$

Where;

Entering $A A D T=$ Sum of the major and minor road AADTs, and

$I C W S=$ Presence of intersection conflict warning systems: 1 if present, 0 if not present.

Tables 5.36 and 5.37 show the coefficient estimates, over dispersion parameters (K) and the goodness of prediction measures for models for various crash severities.

Table 5.36 Estimates and Dispersion Parameters for Four-Lane Model

\begin{tabular}{|c|c|c|c|c|}
\hline \multirow{2}{*}{$\begin{array}{l}\text { Crash Type } \\
\text { Coefficient }\end{array}$} & \multicolumn{2}{|l|}{ Total } & \multicolumn{2}{|l|}{ Injury } \\
\hline & $\begin{array}{c}\text { Estimate } \\
\text { (Standard Error) }\end{array}$ & Pr>ChiSq & $\begin{array}{c}\text { Estimate } \\
\text { (Standard Error) }\end{array}$ & Pr>Chisq \\
\hline$\alpha$ & $-4.3406(0.6909)$ & $<0.0001$ & $-4.0964(0.8515)$ & $<0.0001$ \\
\hline 81 & $0.5676(0.0714)$ & $<0.0001$ & $0.4656(0.0882)$ & $<0.0001$ \\
\hline 82 & $-0.2820(0.1024)$ & 0.0059 & $-0.3894(0.1168)$ & 0.0009 \\
\hline$K$ & \multicolumn{2}{|l|}{0.4854} & \multicolumn{2}{|l|}{0.3420} \\
\hline Crash Type & \multicolumn{2}{|c|}{ Right Angle } & & \\
\hline Coefficient & $\begin{array}{c}\text { Estimate } \\
\text { (Standard Error) }\end{array}$ & Pr>Chisq & & \\
\hline$\alpha$ & $-3.8451(0.9166)$ & $<0.0001$ & & \\
\hline 81 & $0.4470(0.0952)$ & $<0.0001$ & & \\
\hline 82 & $-0.4540(0.1301)$ & 0.0005 & & \\
\hline$K$ & 0.6599 & & & \\
\hline
\end{tabular}


Table 5.37 Goodness of Prediction Measures for Four-Lane Model

\begin{tabular}{|c|c|c|}
\hline Crash Type & Total & Injury \\
\hline \multirow{3}{*}{$\begin{array}{c}\text { Avg. Obs. Crashes/Year/Site } \\
\text { MAD/Year/Site } \\
\text { MPE/Year/Site }\end{array}$} & 2.0447 & 0.9156 \\
\hline & 1.4887 & 0.5291 \\
\hline & 0.0680 & 0.0371 \\
\hline & Side Swipe (OD) & \\
\hline \multirow{3}{*}{$\begin{array}{c}\text { Avg. Obs. Crashes/Year/Site } \\
\text { MAD/Year/Site } \\
\text { MPE/Year/Site }\end{array}$} & 0.9379 & \\
\hline & 0.6426 & \\
\hline & 0.0398 & \\
\hline
\end{tabular}
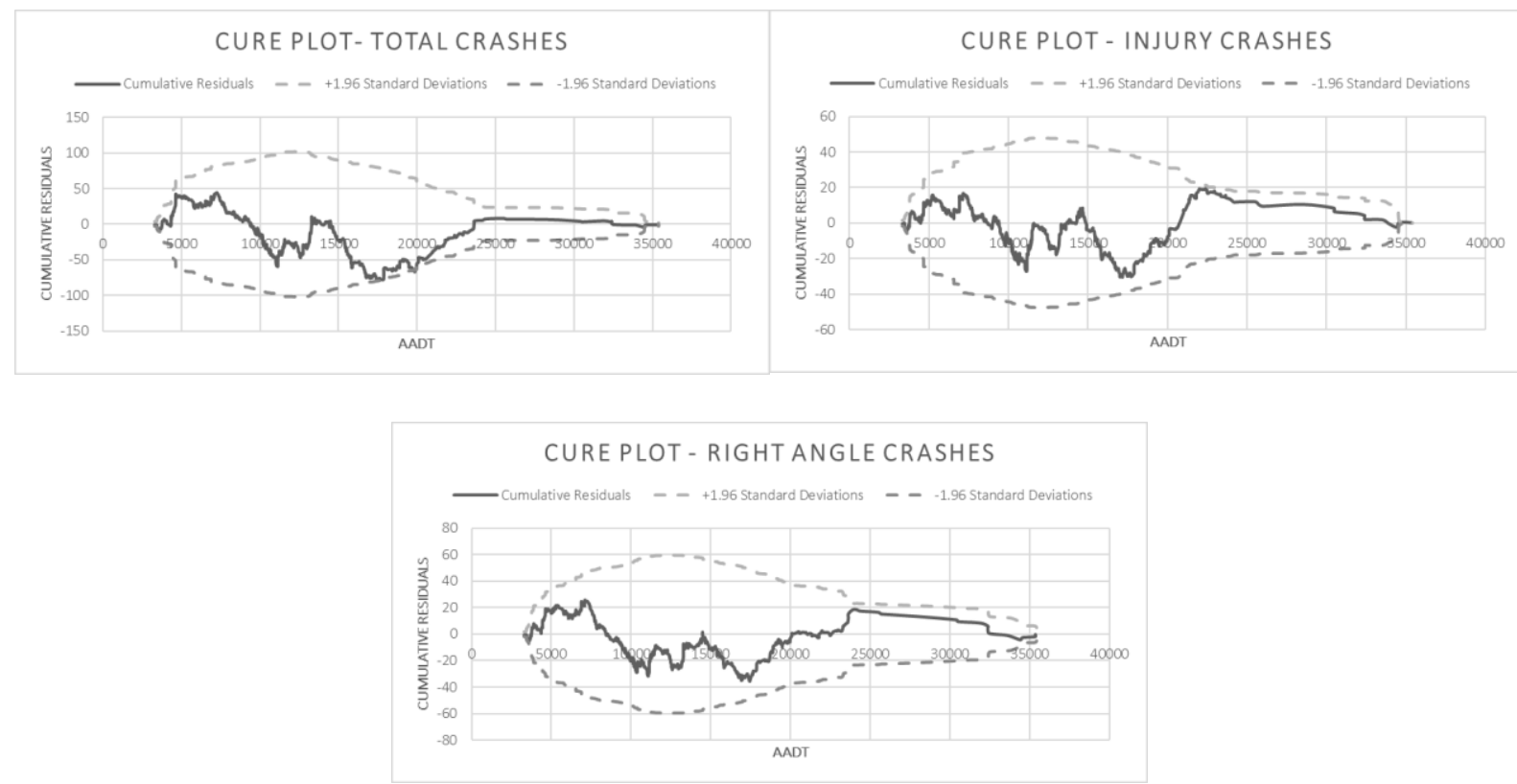

Figure 5.17 CURE Plots for Four-Lane Model

As can be seen from Tables 5.36 and 5.37, the estimates of $\beta_{1}$ and $\beta_{2}$ are highly significant $(\mathrm{P}<0.01)$ for all the models. The goodness of prediction measures also suggests of reasonably good fits in that the MAD/year/site (Mean Absolute Deviation) and the MPE/year/site (Mean Prediction Error) for all the models are small when compared to the average observed crashes per year per site. For example, for total crashes the MAD/year/site is 1.489 and MPE/year/site is 0.068 compared to an average of $\sim 2.05$ total crashes per year per site. 
The CURE plots for all models, shown in Figures 5.17, show that the cumulative residuals lie mostly between the $95 \%$ confidence boundaries and oscillate consistently around the horizontal axis, showing little or no bias.

\subsubsection{Application of Crash Prediction Models for Estimating Crash Modification Factors for Intersection Conflict Warning Systems}

The crash modification factor derived from Equation 5-12 and 5-13 would take the following form:

$C M F=\exp ^{\beta_{x} \times I C W S}$

Where;

$\mathrm{CMF}=$ Crash Modification Factor,

$\beta_{x}=\beta_{2}$ for all model (see Tables 5.34 and 5.36), and

$I C W S=$ Presence of intersection conflict warning systems: 1 if present, 0 if not present.

Tables 5.38 and 5.39 show the CMF estimates from Equation 5-16 for two-lane and four-lane sites and compares them to the CMF estimates from the empirical Bayes study done by Himes et al. (2016) using the dataset used in this chapter. Significance of the differences in the CMF estimates was also tested at the $5 \%$ level of significance (using the two-tailed t-test) and can be seen in Tables 5.38 and 5.39 . 
Table 5.38 Comparison of 2-Lane CMF Estimates with CMFs from Himes et al. (2016)

\begin{tabular}{|c|c|c|}
\hline Crash Type & 2-Lane CMF (SE) & $\begin{array}{c}\text { 2-Lane CMF from Himes } \\
\text { et al. } 2016 \text { (SE) }\end{array}$ \\
\hline Total & $0.677(0.062)$ & $0.730(0.040)$ \\
\hline Injury & $0.570(0.086)$ & $0.700(0.050)$ \\
\hline Right Angle & $0.414(0.092)$ & $0.800(0.050)$ \\
\hline
\end{tabular}

As can be seen from the results in Table 5.38, the cross-sectional models developed in this section estimate slightly larger crash reductions for total and injury crashes, and nearly double the reduction for right angle crashes on two-lane sites (all with high standard errors) compared to the results from Himes et al. (2016). For example, the model estimated a $32 \%$ reduction in total crashes (with a standard error of 0.062 ) compared to $27 \%$ (with a standard error of 0040) by Himes et al. (2016). The standard errors of the CMFs estimates were slightly higher, but, within the realm of reality this suggests that the estimates are closer to the two-lane CMFs estimated by Himes et al. (2016). Himes et al. (2016) in their study, got a $20 \%$ reduction in right angle crashes, whereas, the cross sectional analysis shows a 59\% reduction. This could possibly be due to the limited number of years used for cross sectional analysis. The differences in CMF estimates (compared to Himes et al.) are insignificant for total crashes showing the two estimates for total crashes are statistically comparable for this crash type. Although the differences between estimates for injury and right angle crashes are significant at the 5\% level, it can be seen that the direction of the magnitude of these crash reductions is correct and hence these estimates can be considered reasonably comparable in this sense, if not statistically so. 
Table 5.39 Comparison of 4-Lane CMF Estimates with CMFs from Himes et al. (2016)

\begin{tabular}{|c|c|c|}
\hline Crash Type & 4-Lane CMF (SE) & $\begin{array}{c}\text { 4-Lane CMF from Himes } \\
\text { et al. } 2016 \text { (SE) }\end{array}$ \\
\hline \multirow{3}{*}{$\begin{array}{c}\text { Total } \\
\text { Injury } \\
\text { Right Angle }\end{array}$} & $0.754(0.102)$ & $0.830(0.060)$ \\
\hline & $0.677(0.117)$ & $0.800(0.070)$ \\
\hline & $0.635(0.130)$ & $0.850(0.080)$ \\
\hline
\end{tabular}

Note: Differences between CMFs (for the Crash Type) Statistically Insignificant at 5\% Level shown in boldface

For four-lane sites, the CMFs again follow a similar trend. For example, the model predicts a $25 \%$ reduction in total crashes (with a standard error of 0.102 ) compared to $17 \%$ (with a standard error of 0.060) by Himes et al. (2016). The standard errors of the CMFs estimates were slightly higher, but, within the realm of reality this suggests that the estimates are closer to the CMFs estimated by Himes et al. (2016). The differences in CMF estimates (compared to Himes et al.) are insignificant for total and injury crashes showing the two estimates for these crashes are statistically comparable. Although the difference in estimates for right angle crashes is significant at the $5 \%$ level, it can be seen that the direction of the magnitude of the crash reduction is correct and hence this estimate can be considered reasonably comparable in this sense if not in the strict statistical sense.

Overall, the results from the cross-sectional analysis seem to follow a similar trend to the results from the empirical Bayes study and the estimates are comparable generally comparable to those from the empirical Bayes study. Figure 5.18 shows the comparison between the CMFs in the form of a bar graph for easy visual understanding. The similar trend in the CMF estimates can be seen from the bar graphs. 

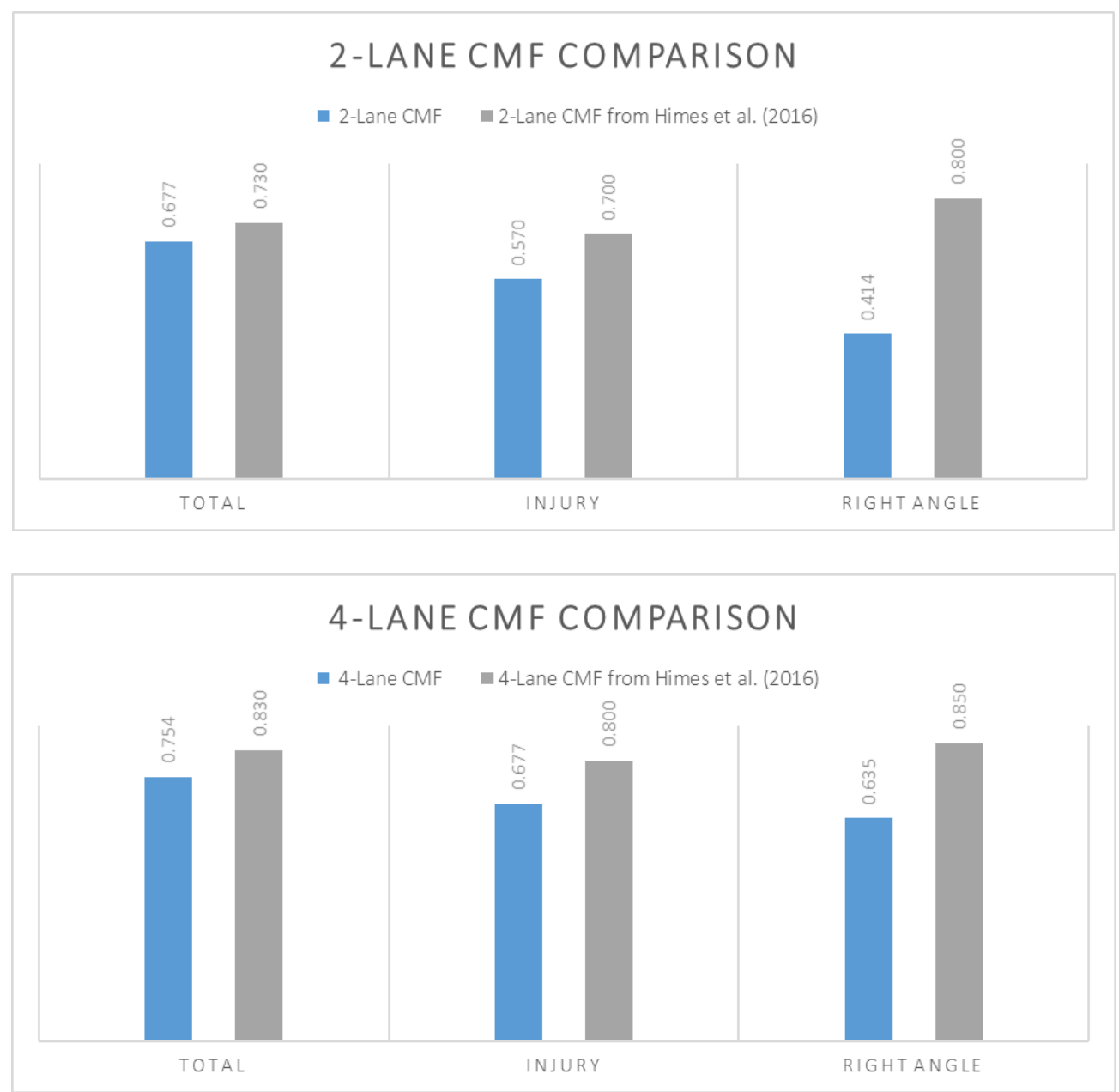

Figure 5.18 Bar Graphs for the Comparison of Two- \& Four-Lane CMFs

\subsubsection{Summary of the Intersection Conflict Warning Case Study}

This section presented the last of the three case studies used to evaluate the consistency of crash modification factors estimated from cross-sectional analysis using before-after data compared to CMFs estimated from empirical Bayes before-after evaluation. Crash prediction models were developed incorporating the presence of intersection conflict warning systems. These models were statistically significant to the 5\% level and had low standard errors in almost all cases. The goodness of prediction measures also suggested of a reasonably good fit. 
To further demonstrate the capabilities of this approach, estimates of CMFs for presence of intersection conflict warning systems were compared to the empirical Bayes evaluation conducted by Himes et al. (2016). The differences in CMF estimates (compared to Himes et al.) were insignificant at the $5 \%$ level in most cases, with the magnitude and direction of the reduction in cases where the differences were significant suggesting of a reasonable estimates. The results found the comparison to be favourable as the CMFs follow a similar trend.

\subsection{Chapter Summary}

This chapter presented the second part of the CMF research component in this dissertation -evaluating the consistency in estimation of CMFs from cross-sectional analysis using beforeafter data. In this evaluation, three case studies were presented using combination (center line plus shoulder) rumble strips, wet reflective pavement markings, and intersection conflict warning conflict warning systems as design elements.

Crash prediction models were developed incorporating (a) the presence of combination (center line plus shoulder) rumble strips, wet reflective pavement markings. and intersection conflict warning conflict warning systems, and (b) the interaction between shoulder widths and the presence of combination rumble strips and number of lanes and the presence of wet reflective pavement markings, respectively. The model parameters were statistically significant to the $5 \%$ level and had low standard errors. The goodness of prediction measures also suggested a good fit.

To further demonstrate the capabilities of this approach, estimates of CMFs for presence of (center line plus shoulder) rumble strips, wet reflective pavement markings. and intersection 
conflict warning conflict warning systems were compared to the empirical Bayes evaluation conducted by Persaud et al. (2016), Lyon et al. (2016), and Himes et al. (2016), respectively, for the three case studies. The standard errors of the CMF estimates from the cross sectional regression were slightly higher than the comparison studies, but, within the realm of reality, this suggests that the estimates are closer to the CMFs estimated by Persaud et al. (2016), Lyon et al. (2016), and Himes et al. (2016). The high standard errors can also be partly explained by the fact that the comparison studies used real life before and after data of the sites and the cross sectional regression used a combination of reference sites and after period treated sites data. The differences in the CMF estimates (compared to Persaud et al., Lyon et al., and Himes et al.) were also insignificant at the 5\% level in most cases, while the magnitude and direction of the reduction in cases where the differences were significant suggesting reasonably comparable estimates. The results also found the safety effects of changing shoulder width in the presence of combination rumble strips and varying number of lanes in the presence of wet reflective pavement markings to increase as the shoulder width/number of lanes increases. These interactions could not be established using the before-after analysis as noted by the authors of these studies.

Based on the three case studies used, it can be seen that the cross sectional models that were developed, using a combination of the reference site data and the after period data at the treatment sites, can not only estimate CMFs that are close and comparable to the CMFs estimated from empirical Bayes before-after studies but also have the potential to better capture the variation of the CMF with certain factors. This also opens the door for further research in 
this area whereby more treatments can be explored to see whether similar results can be achieved. 


\section{Chapter 6. Investigation of New Approaches to Estimating Safety Performance of Roundabouts}

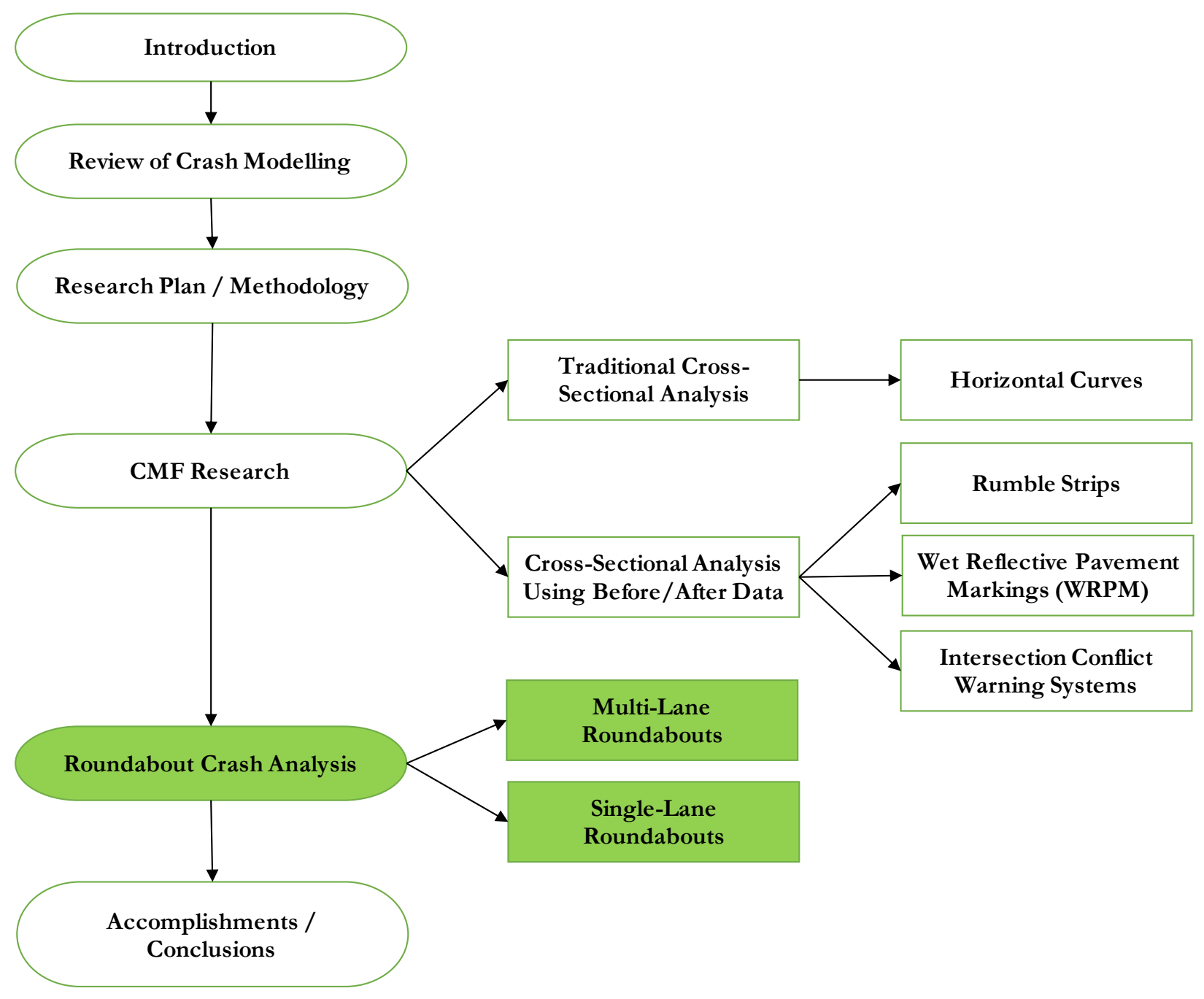

This chapter presents the second component of the research involving a comparative analysis of roundabout crash predictions using conflicting volumes and delays from turning movement counts and the traditional approach flow based models. The purpose is to assess whether it is worth the extra effort to collect turning movement data that are not typically collected at roundabouts. 
The data used came from Region of Waterloo, City of Ottawa, and Washington State. Crash prediction models are developed based on (a) estimated total peak hour conflicting volumes (ETPKCV), (b) circulating flow, (c) entry/exit flow, and (d) total overall delay. ETPKCV was estimated by adding the estimated conflicting volumes for each approach, whereas, the delay (both overall and approach level) were estimated using the highway capacity manual methodology. The relationship between crashes and all the above-mentioned variables was then explored to derive the relationship between safety and traffic operations at roundabouts. This component of research concludes with a comparative assessment of the predictive ability of the various models in order to answer the fundamental question posed - whether it is worthwhile to collect turning movement counts at roundabouts.

\section{$\underline{6.1 \text { Background/Motivation }}$}

Roundabouts are constructed mostly because of their safety and capacity benefits; they provide a solution that can potentially reduce crashes at intersections. According to the National Cooperative Highway Research Program (NCHRP) Report 672: "Roundabouts: An Informational Guide" (TRB, 2010a), roundabouts are shown to reduce injury collisions by approximately $76 \%$ as compared to stop control or traffic signals. The three characteristics of roundabouts that contribute to their increased safety are reduced vehicle speeds on all approaches, reduction in the number of conflict points and the change in the types of crashes that occur (Isebrands, 2011). This is due to the fact that all vehicles are forced to reduce their speeds through geometry and yield to circulating flow upon entry encouraging slow and consistent speeds for all traffic (Isebrands, 2011). 
Pellecuer \& St-Jacques (2008) and Rodegerdts et al. (2007) have shown that roundabouts are steadily gaining popularity in regions where they have not applied in the past. NCHRP Report 672 (TRB, 2010a) found that in a number of circumstances, roundabouts proved to be more beneficial in terms of both operations and safety when compared to other intersection types.

Generally, only entering volumes (commonly referred to as entry/exit flow in the Highway Capacity Manual) are collected at roundabouts. This is due to the roundabout geometry making it difficult and costly to do turning movement counts. As a result, turning movement counts are hardly ever collected for roundabouts even though they are required for both capacity and level of service (LOS) calculations with the Highway Capacity Manual methodology. These counts may also help in getting better safety estimates. This leads to the main motivation behind this component of this research -- whether it is worthwhile to put forward the considerable extra effort needed to collect these data. This is accomplished by investigating new approaches for estimating roundabout safety using conflicting volumes and delays, both of which could only be estimated using the turning movement counts. For this purpose, samples of multi- and single-lane roundabouts from the Region of Waterloo and City of Ottawa in Canada, and Washington State were used. State-of-the-art, generalized linear modeling (GLM), with the specification of a negative binomial (NB) error structure, was used to develop the crash prediction models. Models were developed linking crashes to the estimated total peak hour conflicting volume (ETPKCV) and the overall total roundabout delay.

To demonstrate the versatility of the approach, multi-lane roundabouts (in the Region of Waterloo and Washington State) and single-lane roundabouts (in the City of Ottawa and 
Washington State) were used as separate case studies to assess the validity of the approach. The viability of the approach was further assessed by comparing the developed models within that approach to the conventional models linking crashes to circulating and entry/exit traffic flows.

The reliability of the model estimates was enhanced further by accounting for the variation/trends in crash counts due to the influence of factors that change from year-to-year. This variation was captured by treating the counts for each year as a separate observation using general estimating equations (GEE) to develop crash prediction models accounting for time trend and/or the temporal correlation in crash data.

\subsection{Data and Methodology}

The data for this study were provided by the Region of Waterloo's Transportation Engineering department, City of Ottawa's Transportation Engineering Department, and the Washington State Department of Transportation. The overall data consisted of 19 multi-lane and 22 singlelane roundabouts. These 41 roundabouts were the only ones from the 300 plus roundabouts that were submitted for NCHRP 17-70 project (TRB, 2016) to have turning movement counts, emphasizing the reality that these data are typically not collected.

A total of five years of crash data were used for the Region of Waterloo sites and seven years for the City of Ottawa and Washington State sites. Circulating flows and the entry/exit flows were estimated using the methodologies in Chapter 21 of the Highway Capacity Manual (TRB, 2010). Circulating flow is classified as the entering traffic that will use the traffic circle and does not include right turning traffic using bypass lanes (if available). The entry/exit flow 
for the roundabout is basically the total entering AADT obtained by adding all the flows on major and minor road approaches. "Entry/exit" terminology is used in this research instead of the more common "entering flow" in order to maintain consistency with the terminology in the Highway Capacity Manual (TRB, 2010).

Total peak hour conflicting volumes were estimated by adding the peak hour conflicting volumes (left turning (L), right turning (R), through (T) and total volumes) for each approach. The methodology used to define the conflicting volumes for each approach was developed as an extension to the already defined procedure in the Highway Capacity Manual (TRB, 2010).

Estimated peak hour conflicting volumes for approaches at multi-lane roundabouts were calculated using Equations 6-1 - 6-4.

$\boldsymbol{E B}$ Conflicting Volume $=\min (E B L, S B T)+\min (E B L, S B L)+\min (E B L, N B L)+$ $\min (E B L, N B T)+\min (E B L, W B L)+\min (E B L, W B T)+\min (E B T, S B L)+$ $\min (E B T, S B T)+\min (E B T, N B L)+\min (E B T, N B T)+\min (E B T, N B R)+$ $\min (E B R, S B T)$

WB Conflicting Volume $=\min (W B L, N B L)+\min (W B L, N B T)+$ $\min (W B L, S B L)+\min (W B L, S B T)+\min (W B L, E B L)+\min (W B L, E B T)+$ $\min (W B T, N B L)+\min (W B T, N B T)+\min (W B T, S B L)+\min (W B T, S B T)+$ $\min (W B T, S B R)+\min (W B R, N B T)$

NB Conflicting Volume $=\min (N B L, E B L)+\min (N B L, E B T)+\min (N B L, W B L)+$ $\min (N B L, W B T)+\min (N B L, S B L)+\min (N B L, S B T)+\min (N B T, E B L)+$ 
$\min (N B T, E B T)+\min (N B T, W B L)+\min (N B T, W B T)+\min (N B T, W B R)+$

$\min (N B R, W B T)$

SB Conflicting Volume $=\min (S B L, W B L)+\min (S B L, W B T)+\min (S B L, E B L)+$ $\min (S B L, E B T)+\min (S B L, N B L)+\min (S B L, N B T)+\min (S B T, W B L)+$ $\min (S B T, W B T)+\min (S B T, E B L)+\min (S B T, E B T)+\min (S B T, E B R)+$ $\min (S B R, W B T)$

Similarly, at single-lane roundabouts, the estimated peak hour conflicting volumes for approaches were calculated using Equations 6-5 - 6-8.

EB Conflicting Volume $=\min \left(E B_{\text {Total }}, S B_{\text {Total }}-S B R\right)+\min \left(E B_{\text {Total }}-\right.$ $\left.E B R, N B_{\text {Total }}\right)+\min \left(E B L, W B_{\text {Total }}\right)$

WB Conflicting Volume $=\min \left(W B_{\text {Total }}, N B_{\text {Total }}-N B R\right)+\min \left(W B_{\text {Total }}-\right.$

$\left.W B R, S B_{\text {Total }}\right)+\min \left(W B L, E B_{\text {Total }}\right)$

NB Conflicting Volume $=\min \left(N B_{\text {Total }}, E B_{\text {Total }}-E B R\right)+\min \left(N B_{\text {Total }}-\right.$

$\left.N B R, W B_{\text {Total }}\right)+\min \left(N B L, S B_{\text {Total }}\right)$

SB Conflicting Volume $=\min \left(S B_{\text {Total }}, W B_{\text {Total }}-W B R\right)+\min \left(S B_{\text {Total }}-\right.$

$\left.S B, E B_{\text {Total }}\right)+\min \left(S B L, N B_{\text {Total }}\right)$

The total peak hour conflicting volumes for each roundabout were calculated by adding all of the conflicting flows pertaining to the roundabout in question. The overall roundabout delay per vehicle was calculated using the following equation from the Highway Capacity Manual (TRB, 2010). 
Delay $_{\text {Lane }}=\frac{3600}{c}+900\left(x-1+\sqrt{(x-1)^{2}+\frac{\left(\frac{3600}{c}\right) x}{450}}\right)+5 \times \min [x, 1]$

Where;

$c=$ the capacity of the subject lane, and

$x=$ the volume-to-capacity ratio of the subject lane.

The capacity, $c$, of the subject lane is calculated using Exhibits 21-15 and 21-16 of the Highway Capacity Manual 2010 (TRB, 2010). The average roundabout delay per vehicle was then multiplied by the roundabout volumes to get the total overall roundabout delay. The average delay per vehicle for each lane was calculated using the following equation.

Delay $_{\text {Approach }}=\frac{\sum_{\text {Delay }} \text { Lane } \text { Volume }_{\text {Lane }}}{\sum \text { Volume }_{\text {Lane }}}$

Delay $_{\text {Roundbaout }}=\frac{{\sum \text { Delay }_{\text {Approach }} \text { Volume }_{\text {Approach }}}_{\text {}}}{\sum \text { Volume }_{\text {Approach }}}$

Tables 6.1, 6.2, 6.3 and 6.4 show the summary statistics for the traffic and crash data at the roundabouts used in this study.

Table 6.1 Summary Statistics for Region of Waterloo Multi-Lane Roundabouts

\begin{tabular}{|c|c|c|c|c|}
\hline \multicolumn{5}{|c|}{ Region of Waterloo Multi-Lane Roundabouts (2010-2014) (n=12) } \\
\hline Data & Minimum & Maximum & Mean & Percentage \\
\hline ETPKCV & 940 & 9228 & 3697.68 & - \\
\hline Total Delay (sec) & 14509.00 & 369832.00 & 114000.10 & - \\
\hline Circulating Volume & 1174 & 3566 & 2691.30 & - \\
\hline Entry/Exit Volume & 1174 & 3638 & 2731.86 & - \\
\hline Total Crashes & 13 & 192 & 69.75 & $100 \%$ \\
\hline Injury Crashes & 0 & 22 & 7.33 & $11 \%$ \\
\hline PDO Crashes & 13 & 170 & 62.42 & $89 \%$ \\
\hline
\end{tabular}

Note: ETPKCV = Estimated Total Peak Hour Conflicting Volume 
Table 6.2 Summary Statistics for City of Ottawa Single-Lane Roundabouts

\begin{tabular}{|c|c|c|c|c|}
\hline \multicolumn{5}{|c|}{ City of Ottawa Single-Lane Roundabouts (2006 - 2013) $(n=5)$} \\
\hline Data & Minimum & Maximum & Mean & Percentage \\
\hline \multirow{4}{*}{$\begin{array}{c}\text { ETPKCV } \\
\text { Total Delay (sec) } \\
\text { Circulating Volume } \\
\text { Entry/Exit Volume }\end{array}$} & 222 & 2497 & 1398.80 & - \\
\hline & 9636.00 & 35064.00 & 24092.50 & - \\
\hline & 727 & 1726 & 1254.60 & - \\
\hline & 747 & 1735 & 1326.80 & - \\
\hline \multirow{3}{*}{$\begin{array}{l}\text { Total Crashes } \\
\text { Injury Crashes } \\
\text { PDO Crashes }\end{array}$} & 3 & 33 & 14.20 & $100 \%$ \\
\hline & 0 & 11 & 3.60 & $25 \%$ \\
\hline & 3 & 22 & 10.60 & $75 \%$ \\
\hline
\end{tabular}

Note: $E T P K C V=$ Estimated Total Peak Hour Conflicting Volume

Table 6.3 Summary Statistics for Washington State Multi-Lane Roundabouts

\begin{tabular}{|c|c|c|c|c|}
\hline \multicolumn{5}{|c|}{ Washinton State Multi-Lane Roundabouts (2008-2014) (n=7) } \\
\hline Data & Minimum & Maximum & Mean & Percentage \\
\hline ETPKCV & 546 & 5744 & 2229.00 & - \\
\hline Total Delay (sec) & 3476.00 & 200930.00 & 114000.10 & - \\
\hline Circulating Volume & 622 & 2784 & 1924.29 & - \\
\hline Entry/Exit Volume & 651 & 2938 & 2063.86 & - \\
\hline Total Crashes & 4 & 211 & 79.29 & $100 \%$ \\
\hline Injury Crashes & 0 & 20 & 12.14 & $15 \%$ \\
\hline PDO Crashes & 4 & 194 & 67.14 & $85 \%$ \\
\hline
\end{tabular}

Note: $E T P K C V=$ Estimated Total Peak Hour Conflicting Volume

Table 6.4 Summary Statistics for Region of Waterloo Single-Lane Roundabouts

\begin{tabular}{|c|c|c|c|c|}
\hline \multicolumn{5}{|c|}{ Washington State Single-Lane Roundabouts (2008-2014) (n=17) } \\
\hline Data & Minimum & Maximum & Mean & Percentage \\
\hline ETPKCV & 438 & 2394 & 1213.29 & - \\
\hline Total Delay (sec) & 4161.00 & 136756.00 & 36206.80 & - \\
\hline Circulating Volume & 640 & 2320 & 1294.41 & - \\
\hline Entry/Exit Volume & 749 & 2341 & 1325.59 & - \\
\hline Total Crashes & 2 & 19 & 11.82 & $100 \%$ \\
\hline Injury Crashes & 0 & 5 & 2.12 & $18 \%$ \\
\hline PDO Crashes & 2 & 18 & 9.71 & $82 \%$ \\
\hline
\end{tabular}

Note: ETPKCV = Estimated Total Peak Hour Conflicting Volume

Table 6.5 shows the total crashes summed by years for all the multi- and single-lane sites within the same region. 
Table 6.5 Total Crashes (By Years) Recorded at Sites Used for Analysis

\begin{tabular}{|c|c|c|c|}
\hline \multicolumn{2}{|c|}{$\begin{array}{c}\text { Region of Waterloo Multi-Lane } \\
\text { Roundabouts }\end{array}$} & \multicolumn{2}{|c|}{$\begin{array}{c}\text { City of Ottawa Single-Lane } \\
\text { Roundabouts }\end{array}$} \\
\hline Year & Total Crashes & Year & Total Crashes \\
\hline 2010 & 129 & \multirow{7}{*}{$\begin{array}{l}2007 \\
2008 \\
2009 \\
2010 \\
2011 \\
2012 \\
2013 \\
\end{array}$} & 10 \\
\hline 2011 & 159 & & 5 \\
\hline 2012 & 189 & & 8 \\
\hline 2013 & 180 & & 7 \\
\hline 2014 & 180 & & 15 \\
\hline & & & 12 \\
\hline & & & 8 \\
\hline \multicolumn{2}{|c|}{$\begin{array}{l}\text { Washington State Multi-Lane } \\
\text { Roundabouts }\end{array}$} & \multicolumn{2}{|c|}{$\begin{array}{c}\text { Washington State Single-Lane } \\
\text { Roundabouts }\end{array}$} \\
\hline Year & Total Crashes & Year & Total Crashes \\
\hline 2008 & 74 & \multirow{2}{*}{$\begin{array}{l}2008 \\
2009\end{array}$} & 15 \\
\hline 2009 & 71 & & 25 \\
\hline 2010 & 80 & \multirow{2}{*}{$\begin{array}{l}2010 \\
2011\end{array}$} & 43 \\
\hline 2011 & 66 & & 21 \\
\hline 2012 & 83 & 2012 & 20 \\
\hline 2013 & 90 & 2013 & 35 \\
\hline 2014 & 91 & 2014 & 42 \\
\hline
\end{tabular}

\subsection{Model Fitting and Evaluation}

Consistent with state-of-the-art methods, generalized linear modeling (GLM), with the specification of a negative binomial (NB) error structure, was used to develop the crash prediction models (Persaud et al., 2012) using the SAS software (SAS, 2016).

\subsubsection{Multi-Lane Roundabout Models}

The multi-lane roundabout data from Region of Waterloo and Washington State were used to develop models linking crashes to estimated total peak hour conflicting volumes (ETPKCV), total overall roundabout delay, circulating flow, and entry/exit flow. The model form used for developing these models are shown below in Equations 6-12 - 6-15. 
Crashes $=e^{\alpha} \times E T P K C V^{\beta_{1}} \times$ Years

Crashes $=e^{\alpha} \times$ Total Delay ${ }^{\beta_{1}} \times$ Years

Crashes $=e^{\alpha} \times$ Circulating Flow ${ }^{\beta_{1}} \times$ Years

Crashes $=e^{\alpha} \times$ Entry $/$ Exit Flow ${ }^{\beta_{1}} \times$ Years

These models are evaluated in the following sections for total, injury and PDO crashes.

\subsubsection{Total Crash Models}

The model forms shown in Equations 6-12 - 6-15 were used for developing the various total crash models. Table 6.6 shows the coefficient estimates, over dispersion parameter $(\mathrm{K})$ and the goodness of prediction measures for total crash models distinguished by different model types. The CURE plots for all the model types are shown in Figure 6.1.

Table 6.6 Estimates and Goodness of Fit Measures for Total Crash Models

\begin{tabular}{|c|c|c|c|c|}
\hline Model & \multicolumn{2}{|c|}{ ETPKCV } & \multicolumn{2}{|c|}{ Delay } \\
\hline Coefficient & $\begin{array}{c}\text { Estimate } \\
\text { (Standard Error) }\end{array}$ & Pr>ChiSq & $\begin{array}{c}\text { Estimate } \\
\text { (Standard Error) }\end{array}$ & Pr>ChiSq \\
\hline$\alpha$ & $-4.9954(0.8758)$ & $<0.0001$ & $-2.2756(0.8972)$ & 0.0230 \\
\hline 81 & $0.9566(0.1109)$ & $<0.0001$ & $0.4360(0.1730)$ & 0.0117 \\
\hline$K$ & \multicolumn{2}{|c|}{0.0911} & \multicolumn{2}{|c|}{0.4777} \\
\hline Model & \multicolumn{2}{|c|}{ Circulating Flow } & \multicolumn{2}{|c|}{ Entry/Exit Flow } \\
\hline Coefficient & $\begin{array}{c}\text { Estimate } \\
\text { (Standard Error) }\end{array}$ & Pr>ChiSq & $\begin{array}{c}\text { Estimate } \\
\text { (Standard Error) }\end{array}$ & Pr>ChiSq \\
\hline$\alpha$ & $-9.6756(2.2504)$ & $<0.0001$ & $-9.9580(2.3541)$ & $<0.0001$ \\
\hline 81 & $1.5805(0.2902)$ & $<0.0001$ & $1.6106(0.3023)$ & $<0.0001$ \\
\hline$K$ & \multicolumn{2}{|c|}{0.2415} & \multicolumn{2}{|c|}{0.2518} \\
\hline Model & ETPKCV & Delay & $\begin{array}{c}\text { Circulating } \\
\text { Flow }\end{array}$ & Entry/Exit Flow \\
\hline Avg. Obs. Crashes/Year/Site & 14.6560 & 14.6560 & 14.6560 & 14.6560 \\
\hline MAD/Year/Site & 9.5140 & 6.6506 & 5.7996 & 8.5003 \\
\hline MPE/Year/Site & 7.8674 & 5.0076 & 4.1955 & 5.3615 \\
\hline
\end{tabular}



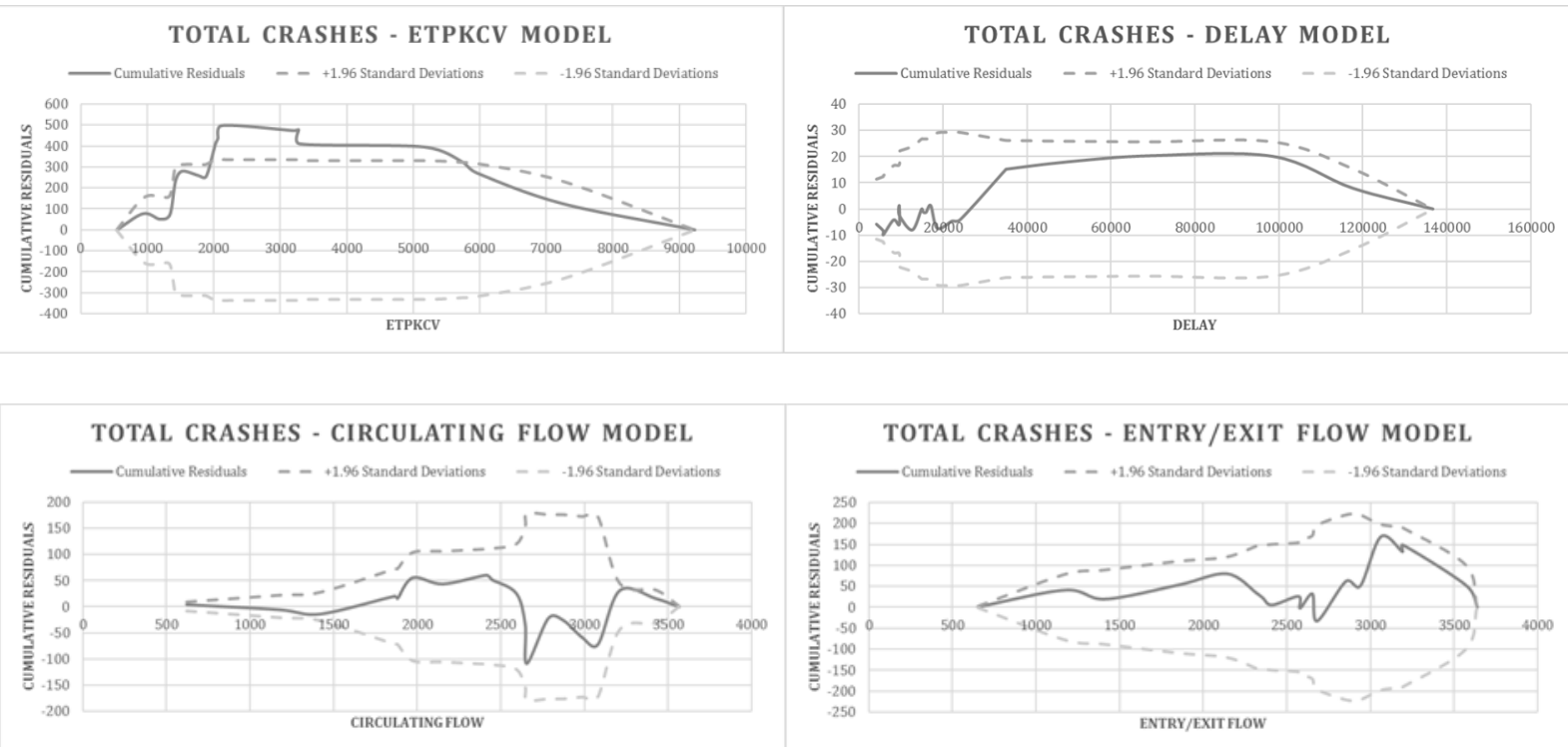

Figure 6.1 CURE Plots for all Model Types (Total Crashes)

As can be seen from the p-values in Table 6.6, the coefficient estimates are highly significant for all the models. The goodness of prediction measures also show that the MAD/year/site (Mean Absolute Deviation) and the MPE/year/site (Mean Prediction Error) for all the models are small when compared to the average observed crashes per year per site. For example, for ETPKCV model the MAD/year/site is 9.51 compared to average observed total crashes of $\sim 15$ per year per site. Even though these values are higher than what one would expect them to ideally be, they are comparable to the values obtained by both the circulating flow and the entry/exit flow models. It can also be seen that the ETPKCV model has a low $\mathrm{K}$ value compared to both of the flow based models ( 0.09 compared to $0.24 \& 0.25$ ) showing that the ETPKCV model is better at capturing the over dispersion in the data compared to the flow based models.

The CURE plots (Figure 6.1) for all of the delay and the flow based models show that the cumulative residuals lie between the $95 \%$ confidence boundaries and oscillate consistently 
showing little or no bias. The oscillation in these plots is not as one would expect in a perfect CURE plot as described by Hauer (2014), but the main reason for that is the smaller number of sites being used here. The CURE plots for ETPKCV model show that the cumulative residuals lie between the $95 \%$ confidence boundaries for smaller and larger values of the conflicting volumes and slight over prediction for conflicting volumes in the range of $\sim 2000$ 5000 ( $\sim 57 \%$ of the multi-lane sites lie in this range). But nonetheless, the MAD, MPE, K, and p-values suggest of a good fit and are an indicator of reasonable predictions by the ETPKCV model for total crashes at multi-lane roundabouts.

\subsubsection{Injury Crash Models}

The model forms shown in Equations 6-12 - 6-15 were used for developing the various injury crash models. Table 6.7 shows the coefficient estimates, over dispersion parameter $(\mathrm{K})$ and the goodness of prediction measures for injury crash models distinguished by different model types. The CURE plots for all model types are shown in Figure 6.2.

As can be seen from the p-values in Table 6.7, the coefficient estimates are highly significant for all the models. The goodness of prediction measures also show that the MAD/year/site (Mean Absolute Deviation) and the MPE/year/site (Mean Prediction Error) for all the models are small when compared to the average observed crashes per year per site. For example, for ETPKCV model the MAD/year/site is 1.06 compared to average observed injury crashes of $\sim 2$ per year per site. Even though these values are higher than what one would expect them to ideally be, they are comparable to the values obtained by both the circulating flow and the entry/exit flow models. It can also be seen that the ETPKCV model has a low $\mathrm{K}$ value 
compared to both of the flow based models ( 0.16 compared to $0.21 \& 0.18)$ showing that the ETPKCV model is better at capturing the over dispersion in the data compared to the flow based models.

Table 6.7 Estimates and Goodness of Fit Measures for Injury Crash Models

\begin{tabular}{|c|c|c|c|c|}
\hline Model & \multicolumn{2}{|c|}{ ETPKCV } & \multicolumn{2}{|c|}{ Delay } \\
\hline Coefficient & $\begin{array}{c}\text { Estimate } \\
\text { (Standard Error) }\end{array}$ & Pr>ChiSq & $\begin{array}{c}\text { Estimate } \\
\text { (Standard Error) }\end{array}$ & Pr>ChiSq \\
\hline$\alpha$ & $-2.7403(1.2988)$ & 0.0349 & $-1.2283(0.6598)$ & 0.0459 \\
\hline 81 & $0.4199(0.1641)$ & 0.0105 & $0.1529(0.0510)$ & 0.0311 \\
\hline$K$ & \multicolumn{2}{|c|}{0.1654} & \multicolumn{2}{|c|}{0.3717} \\
\hline Model & \multicolumn{2}{|c|}{ Circulating Flow } & \multicolumn{2}{|c|}{ Entry/Exit Flow } \\
\hline Coefficient & $\begin{array}{c}\text { Estimate } \\
\text { (Standard Error) }\end{array}$ & Pr>ChiSq & $\begin{array}{c}\text { Estimate } \\
\text { (Standard Error) }\end{array}$ & Pr>ChiSq \\
\hline$\alpha$ & $-4.8105(2.5607)$ & 0.0603 & $-5.7718(1.5653)$ & $<0.0001$ \\
\hline 81 & 0.6939 (0.3299) & 0.0354 & $0.8132(0.2284)$ & $<0.0001$ \\
\hline$K$ & \multicolumn{2}{|c|}{0.2085} & \multicolumn{2}{|c|}{0.1795} \\
\hline Crash Type & ETPKCV & Delay & $\begin{array}{c}\text { Circulating } \\
\text { Flow }\end{array}$ & Entry/Exit Flow \\
\hline \multirow{3}{*}{$\begin{array}{c}\text { Avg. Obs. Crashes/Year/Site } \\
\text { MAD/Year/Site } \\
\text { MPE/Year/Site }\end{array}$} & 1.7540 & 1.7540 & 1.7540 & 1.7540 \\
\hline & 1.0644 & 0.7372 & 0.8710 & 0.8699 \\
\hline & 0.6629 & 0.5279 & 0.5222 & 0.5602 \\
\hline
\end{tabular}
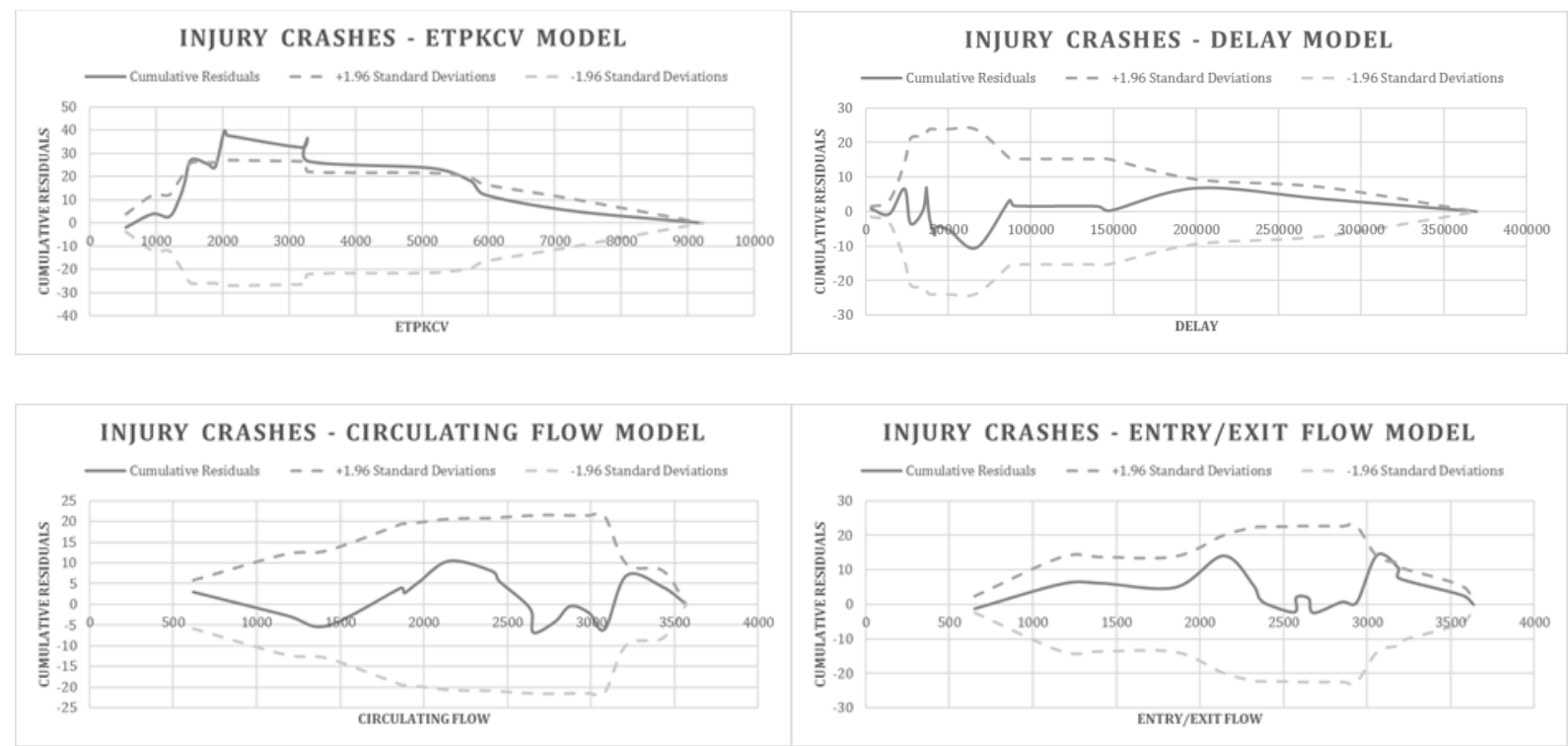

Figure 6.2 CURE Plots for all Model Types (Injury Crashes) 
The CURE plots (Figure 6.2) for all of the delay and the flow based models show that the cumulative residuals lie between the $95 \%$ confidence boundaries and oscillate consistently showing little or no bias. The oscillation in these plots is not as one would expect in a perfect CURE plot as described by Hauer (2014), but the main reason for that is, as before, the smaller number of sites being used here. The CURE plots for ETPKCV model show that the cumulative residuals lie between the $95 \%$ confidence boundaries for smaller and larger values of the conflicting volumes and slight over prediction for conflicting volumes in the range of $\sim 2000-5000(\sim 57 \%$ of the multi-lane sites lie in this range). But nonetheless, the MAD, MPE, $\mathrm{K}$, and $\mathrm{p}$-values suggest of a good fit and are an indicator of reasonable predictions by the ETPKCV model for injury crashes at multi-lane roundabouts.

\subsubsection{PDO Crash Models}

The model forms shown in Equations 6-12 - 6-15 were used for developing the various PDO crash models. Table 6.8 shows the coefficient estimates, over dispersion parameter $(\mathrm{K})$ and the goodness of prediction measures for PDO crash models distinguished by different model types. The CURE plots for all the model types are shown in Figure 6.3.

As can be seen from the p-values in Table 6.8, the coefficient estimates are highly significant for all the models. The goodness of prediction measures also show that the MAD/year/site (Mean Absolute Deviation) and the MPE/year/site (Mean Prediction Error) for all models are small when compared to the average observed crashes per year per site. For example, for ETPKCV model the MAD/year/site is 8.66 compared to average observed PDO crashes of $\sim 12.90$ per year per site. Even though these values are higher than what one would expect them 
to ideally be, they are comparable to the values obtained by both the circulating flow and the entry/exit flow models. It can also be seen that the ETPKCV model has a low $\mathrm{K}$ value compared to both of the flow based models ( 0.08 compared to $0.25 \& 0.27$ ) showing that the ETPKCV model is better at capturing the over dispersion in the data compared to the flow based models.

Table 6.8 Estimates and Goodness of Fit Measures for PDO Crash Models

\begin{tabular}{|c|c|c|c|c|}
\hline Model & \multicolumn{2}{|c|}{ ETPKCV } & \multicolumn{2}{|c|}{ Delay } \\
\hline Coefficient & $\begin{array}{c}\text { Estimate } \\
\text { (Standard Error) }\end{array}$ & Pr>ChiSq & $\begin{array}{c}\text { Estimate } \\
\text { (Standard Error) }\end{array}$ & Pr>ChiSq \\
\hline$\alpha$ & $-5.9080(0.8835)$ & $<0.0001$ & $-3.1679(1.0631)$ & 0.1247 \\
\hline 81 & $1.0521(0.1115)$ & $<0.0001$ & $0.5043(0.1881)$ & 0.0073 \\
\hline$K$ & \multicolumn{2}{|c|}{0.0832} & \multicolumn{2}{|c|}{0.5107} \\
\hline Model & \multicolumn{2}{|c|}{ Circulating Flow } & \multicolumn{2}{|c|}{ Entry/Exit Flow } \\
\hline Coefficient & $\begin{array}{c}\text { Estimate } \\
\text { (Standard Error) }\end{array}$ & Pr>ChiSq & $\begin{array}{c}\text { Estimate } \\
\text { (Standard Error) }\end{array}$ & Pr>ChiSq \\
\hline$\alpha$ & $-11.7657(2.4803)$ & $<0.0001$ & $-11.9665(2.6382)$ & $<0.0001$ \\
\hline 81 & $1.8300(0.3195)$ & $<0.0001$ & $1.8492(0.3385)$ & $<0.0001$ \\
\hline$K$ & \multicolumn{2}{|c|}{0.2535} & \multicolumn{2}{|c|}{0.2741} \\
\hline Crash Type & ETPKCV & Delay & $\begin{array}{c}\text { Circulating } \\
\text { Flow }\end{array}$ & Entry/Exit Flow \\
\hline \multirow{3}{*}{$\begin{array}{c}\text { Avg. Obs. Crashes/Year/Site } \\
\text { MAD/Year/Site } \\
\text { MPE/Year/Site }\end{array}$} & 12.9020 & 12.9020 & 12.9020 & 12.9020 \\
\hline & 8.6620 & 6.2612 & 5.3382 & 8.0203 \\
\hline & 7.4379 & 4.7469 & 3.8169 & 5.0268 \\
\hline
\end{tabular}



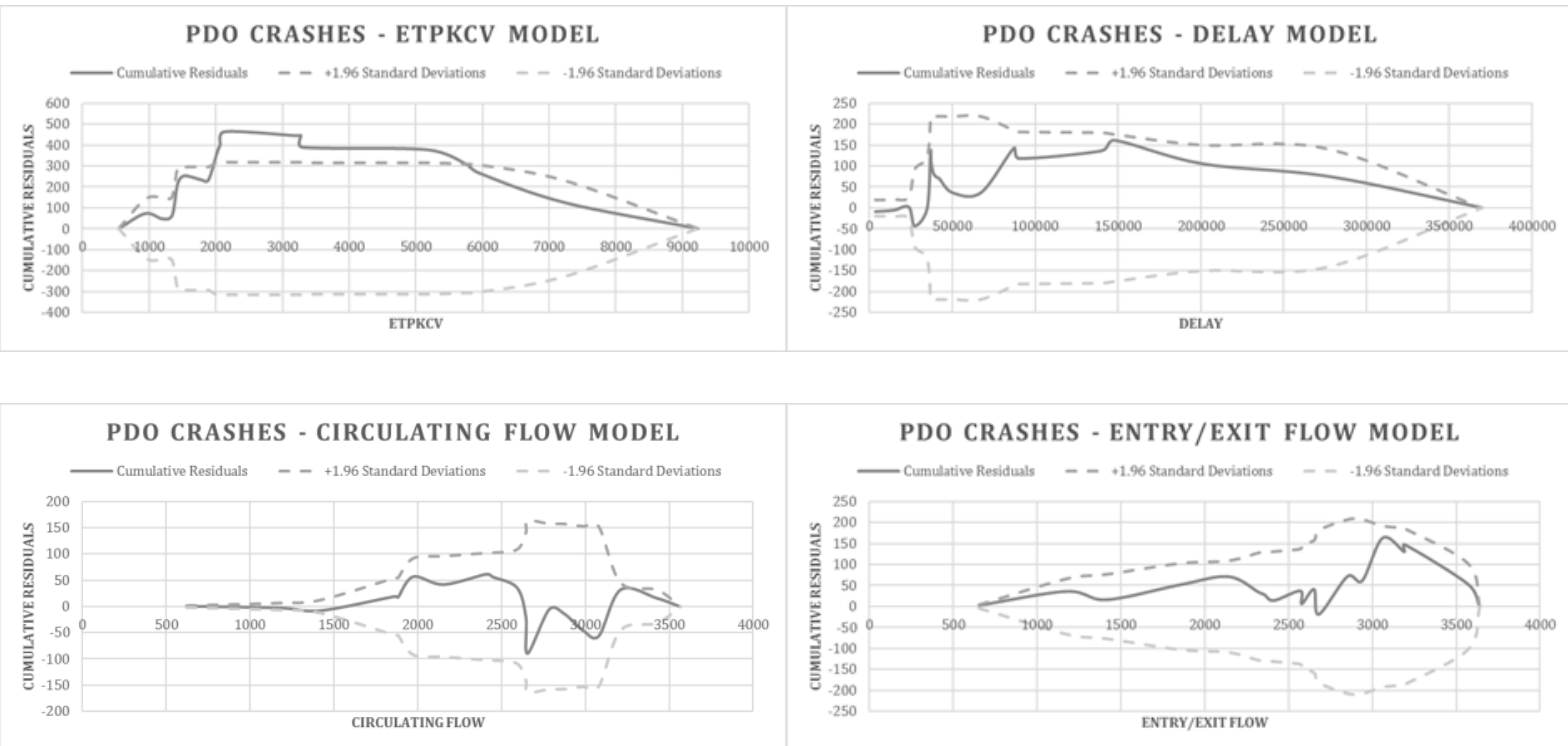

Figure 6.3 CURE Plots for all Model Types (PDO Crashes)

The CURE plots (Figure 6.3) for all of the delay and the flow based models show that the cumulative residuals lie between the $95 \%$ confidence boundaries and oscillate consistently showing little or no bias. The oscillation in these plots is not as one would expect in a perfect CURE plot as described by Hauer (2014), but the main reason for that is, again, the smaller number of sites being used here. The CURE plots for ETPKCV model show that the cumulative residuals lie between the $95 \%$ confidence boundaries for smaller and larger values of the conflicting volumes and slight over prediction for conflicting volumes in the range of $\sim 2000-5000(\sim 57 \%$ of the multi-lane sites lie in this range). But nonetheless, the MAD, MPE, $\mathrm{K}$, and $\mathrm{p}$-values suggest of a good fit and are an indicator of reasonable predictions by the ETPKCV model for PDO crashes at multi-lane roundabouts. 


\subsubsection{Single-Lane Roundabout Models}

The single-lane roundabout data from City of Ottawa and Washington State were used to develop models linking crashes to estimated total peak hour conflicting volumes (ETPKCV), total overall roundabout delay, circulating flow, and entry/exit flow. The model form used for developing these models are shown below in Equations 6-16 - 6-19.

Crashes $=e^{\alpha} \times$ ETPKCV $^{\beta_{1}} \times$ Years

Crashes $=e^{\alpha} \times$ Total Delay $\beta_{1} \times$ Years

Crashes $=e^{\alpha} \times$ Circulating Flow ${ }^{\beta_{1}} \times$ Years

Crashes $=e^{\alpha} \times$ Entry $/$ Exit Flow ${ }^{\beta_{1}} \times$ Years

These models are evaluated in the following sections for total, injury and PDO crashes.

\subsubsection{Total Crash Models}

The model forms shown in Equations 6-16-6-19 were used for developing the various total crash models. Table 6.9 shows the coefficient estimates, over dispersion parameter $(\mathrm{K})$ and the goodness of prediction measures for total crash models distinguished by different model types. The CURE plots for all the model types are shown in Figure 6.4. 
Table 6.9 Estimates and Goodness of Fit Measures for Total Crash Models

\begin{tabular}{|c|c|c|c|c|}
\hline Model & \multicolumn{2}{|c|}{ ETPKCV } & \multicolumn{2}{|c|}{ Delay } \\
\hline Coefficient & $\begin{array}{c}\text { Estimate } \\
\text { (Standard Error) }\end{array}$ & Pr>ChiSq & $\begin{array}{c}\text { Estimate } \\
\text { (Standard Error) }\end{array}$ & Pr>ChiSq \\
\hline$\alpha$ & $-1.4675(0.9238)$ & 0.1122 & $-2.2160(1.2096)$ & 0.0788 \\
\hline 81 & $0.3322(0.1295)$ & 0.0103 & $0.2688(0.1207)$ & 0.0259 \\
\hline$K$ & \multicolumn{2}{|c|}{0.0336} & \multicolumn{2}{|c|}{0.1753} \\
\hline Model & \multicolumn{2}{|c|}{ Circulating Flow } & \multicolumn{2}{|c|}{ Entry/Exit Flow } \\
\hline Coefficient & $\begin{array}{c}\text { Estimate } \\
\text { (Standard Error) }\end{array}$ & Pr>ChiSq & $\begin{array}{c}\text { Estimate } \\
\text { (Standard Error) }\end{array}$ & Pr>ChiSq \\
\hline$\alpha$ & $-3.5490(1.4957)$ & 0.0177 & $-3.6861(1.6216)$ & 0.0230 \\
\hline 81 & $0.6192(0.2080)$ & 0.0029 & $0.6352(0.2246)$ & 0.0047 \\
\hline$K$ & \multicolumn{2}{|c|}{0.0291} & \multicolumn{2}{|c|}{0.0331} \\
\hline Crash Type & ETPKCV & Delay & $\begin{array}{c}\text { Circulating } \\
\text { Flow }\end{array}$ & Entry/Exit Flow \\
\hline \multirow{3}{*}{$\begin{array}{c}\text { Avg. Obs. Crashes/Year/Site } \\
\text { MAD/Year/Site } \\
\text { MPE/Year/Site }\end{array}$} & 2.3373 & 2.3373 & 2.3373 & 2.3373 \\
\hline & 0.6512 & 0.6660 & 0.6474 & 0.6605 \\
\hline & 0.3947 & 0.5218 & 0.3933 & 0.3970 \\
\hline
\end{tabular}

As can be seen from the p-values in Table 6.9, the coefficient estimates are highly significant for all models. The goodness of prediction measures also show that the MAD/year/site (Mean Absolute Deviation) and the MPE/year/site (Mean Prediction Error) for all the models are small when compared to the average observed crashes per year per site. For example, for ETPKCV model the MAD/year/site is 0.65 compared to average observed total crashes of $\sim 2$ per year per site. Even though these values are higher than what one would expect them to ideally be, they are comparable to the values obtained by both the circulating flow and the entry/exit flow models. It can also be seen that the ETPKCV model has $\mathrm{K}$ value that is approximately the same as the flow based models showing that the ETPKCV model can capture the over dispersion in the data comparably to the flow based models. 

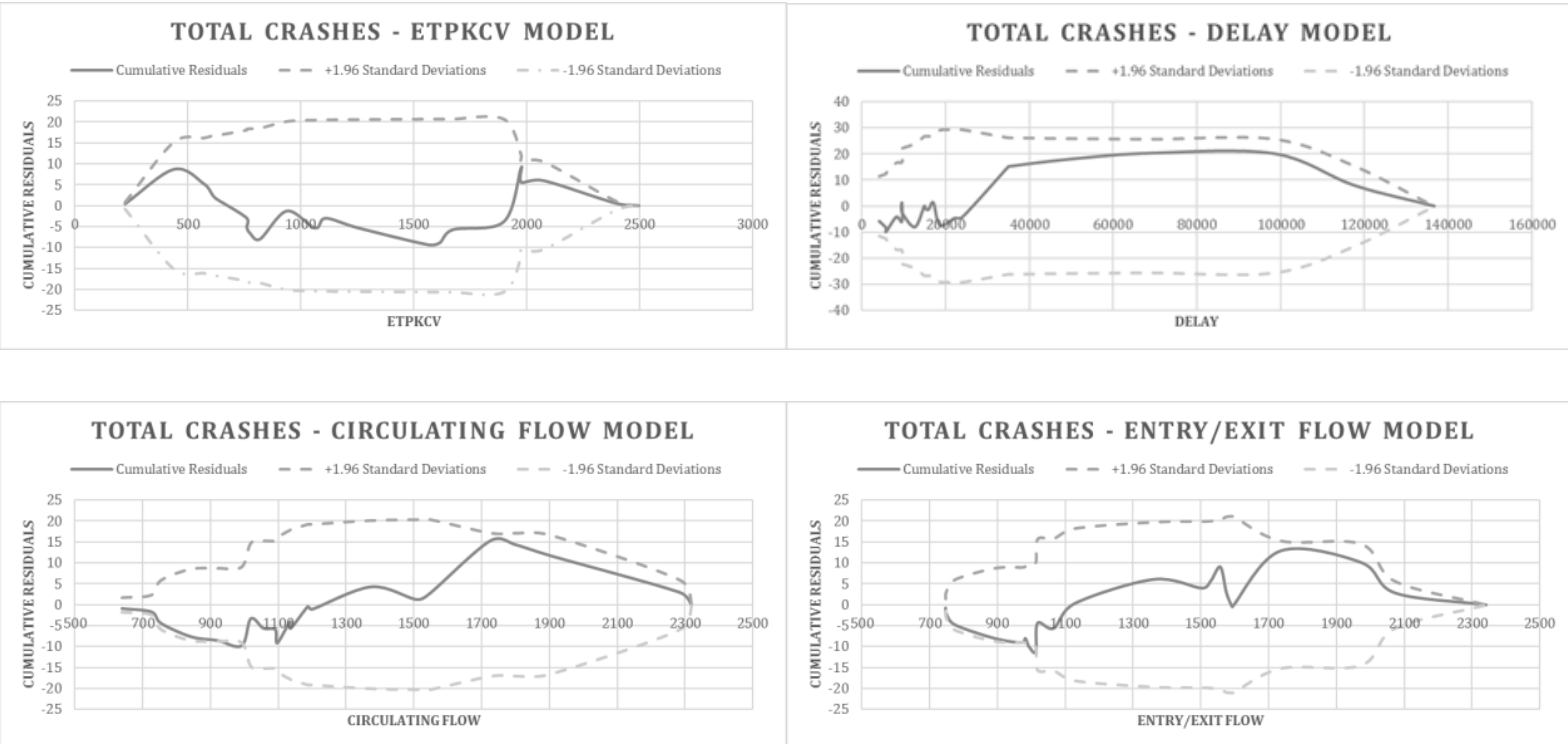

Figure 6.4 CURE Plots for all Model Types (Total Crashes)

The CURE plots (Figure 6.4) for all models show that the cumulative residuals lie between the 95\% confidence boundaries and oscillate consistently showing little or no bias. The oscillation in these plots is not as one would expect in a perfect CURE plot as described by Hauer (2014), but the main reason for that is, once again, the smaller number of sites being used here. Both the goodness of prediction measures and the CURE plots for the models are an indicator of good predictions by all the models.

\subsubsection{Injury Crash Models}

The model forms shown in Equations 6-16 - 6-19 were used for developing the various injury crash models. Table 6.10 shows the coefficient estimates, over dispersion parameter $(\mathrm{K})$ and the goodness of prediction measures for injury crash models distinguished by different model types. The CURE plots for all the model types are shown in Figure 6.5. 
Table 6.10 Estimates and Goodness of Fit Measures for Injury Crash Models

\begin{tabular}{|c|c|c|c|c|}
\hline Model & \multicolumn{2}{|c|}{ ETPKCV } & \multicolumn{2}{|c|}{ Delay } \\
\hline Coefficient & $\begin{array}{c}\text { Estimate } \\
\text { (Standard Error) }\end{array}$ & Pr>ChiSq & $\begin{array}{c}\text { Estimate } \\
\text { (Standard Error) }\end{array}$ & Pr>ChiSq \\
\hline$\alpha$ & $-1.5913(0.9945)$ & 0.0425 & $-2.7783(1.1642)$ & 0.0992 \\
\hline 61 & $0.1202(0.0817)$ & 0.0697 & $0.1732(0.0959)$ & 0.0423 \\
\hline$K$ & \multicolumn{2}{|c|}{0.2268} & \multicolumn{2}{|c|}{0.4589} \\
\hline Model & \multicolumn{2}{|c|}{ Circulating Flow } & \multicolumn{2}{|c|}{ Entry/Exit Flow } \\
\hline Coefficient & $\begin{array}{c}\text { Estimate } \\
\text { (Standard Error) }\end{array}$ & Pr>ChiSq & $\begin{array}{c}\text { Estimate } \\
\text { (Standard Error) }\end{array}$ & Pr>ChiSq \\
\hline$\alpha$ & $-1.8736(0.6605)$ & 0.1634 & $-1.4997(0.7999)$ & 0.0931 \\
\hline 81 & $0.1583(0.0848)$ & 0.1539 & $0.1054(0.0295)$ & 0.0823 \\
\hline$K$ & \multicolumn{2}{|c|}{0.2317} & \multicolumn{2}{|c|}{0.2334} \\
\hline Crash Type & ETPKCV & Delay & $\begin{array}{c}\text { Circulating } \\
\text { Flow }\end{array}$ & Entry/Exit Flow \\
\hline \multirow{3}{*}{$\begin{array}{c}\text { Avg. Obs. Crashes/Year/Site } \\
\text { MAD/Year/Site } \\
\text { MPE/Year/Site }\end{array}$} & 0.4413 & 0.4413 & 0.4413 & 0.4413 \\
\hline & 0.2996 & 0.2325 & 0.3012 & 0.2998 \\
\hline & 0.1856 & 0.1908 & 0.1864 & 0.1866 \\
\hline
\end{tabular}

As can be seen from the p-values in Table 6.10, the coefficient estimates are highly significant for all the models. The goodness of prediction measures also show that the MAD/year/site (Mean Absolute Deviation) and the MPE/year/site (Mean Prediction Error) for all the models are small when compared to the average observed crashes per year per site. For example, for ETPKCV model the MAD/year/site is 0.29 compared to average observed injury crashes of $\sim 0.5$ per year per site. Even though these values are higher than what one would expect them to ideally be, they are comparable to the values obtained by both the circulating flow and the entry/exit flow models. It can also be seen that the ETPKCV model has a slightly lower K value compared to both of the flow based models $(0.22$ compared to $0.23 \& 0.23)$ showing that the ETPKCV model is slightly better at capturing the over dispersion in the data compared to the flow based models. 

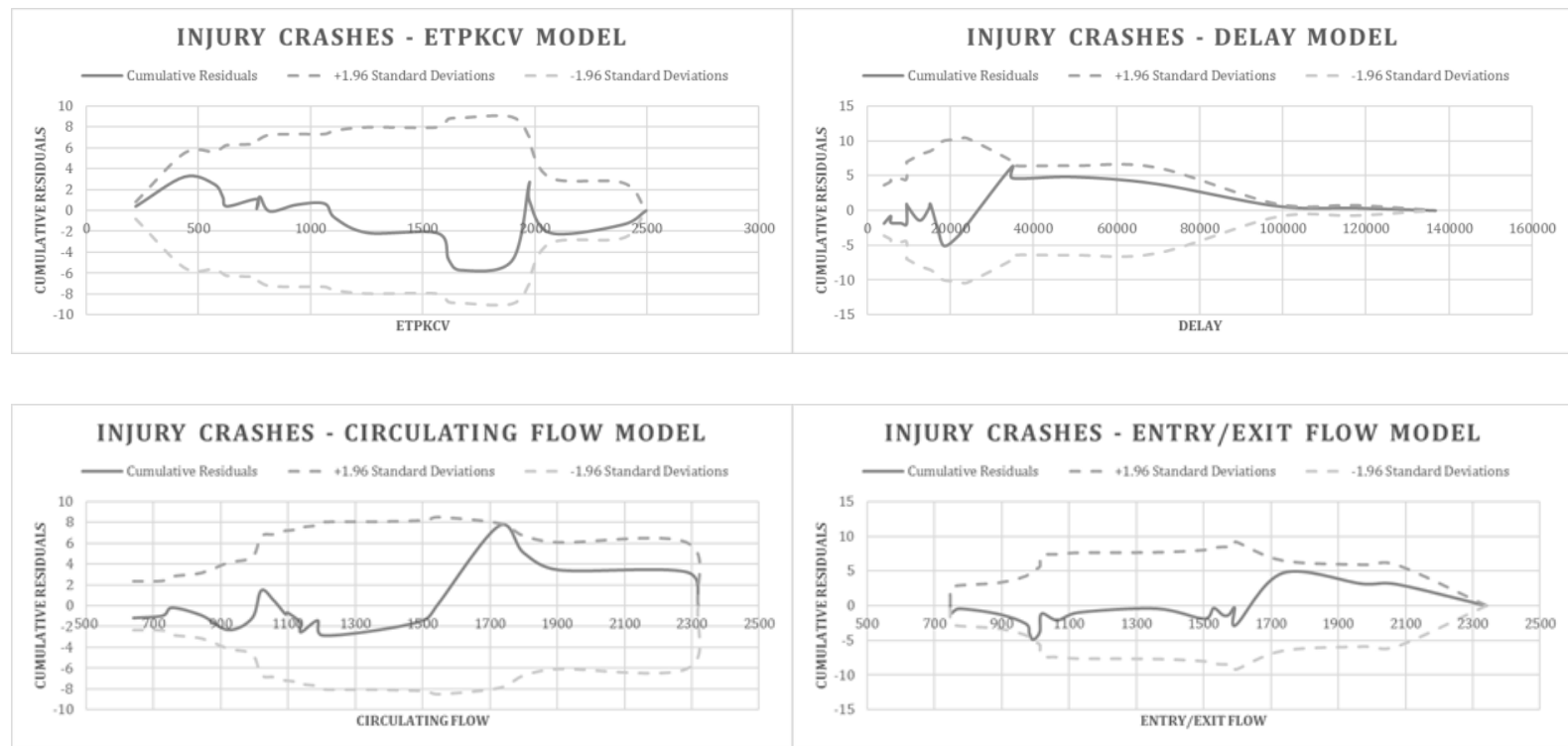

Figure 6.5 CURE Plots for all Model Types (Injury Crashes)

The CURE plots (Figure 6.5) for all models show that the cumulative residuals lie between the 95\% confidence boundaries and oscillate consistently showing little or no bias. The oscillation in these plots is not as one would expect in a perfect CURE plot as described by Hauer (2014), but the main reason for that is, again, the smaller number of sites being used here. Both the goodness of prediction measures and the CURE plots for the models are an indicator of good predictions by all the models.

\subsubsection{PDO Crash Models}

The model forms shown in Equations 6-16 - 6-19 were used for developing the various PDO crash models. Table 6.11 shows the coefficient estimates, over dispersion parameter $(\mathrm{K})$ and the goodness of prediction measures for PDO crash models distinguished by different model types. The CURE plots for all the model types are shown in Figure 6.6. 
Table 6.11 Estimates and Goodness of Fit Measures for PDO Crash Models

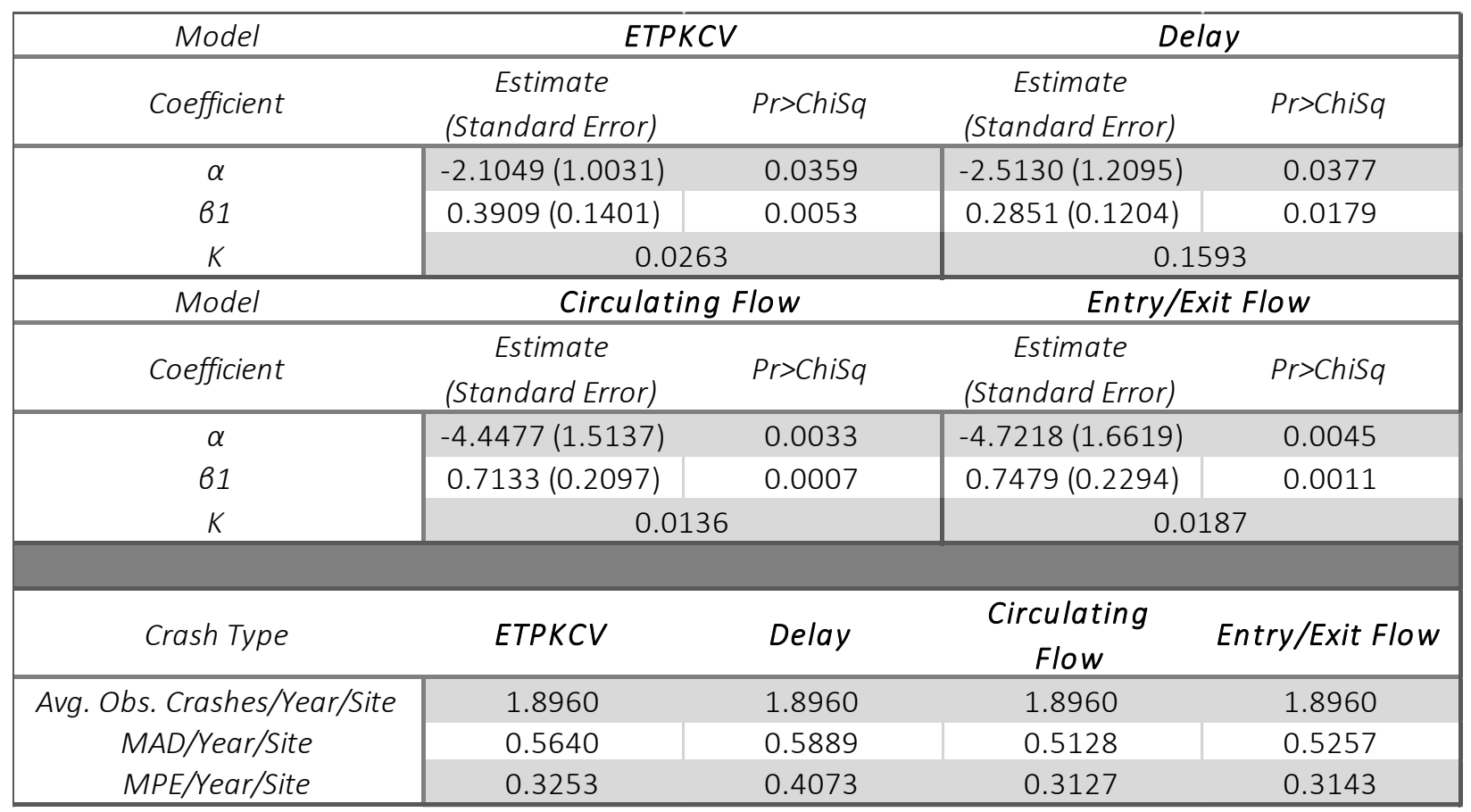
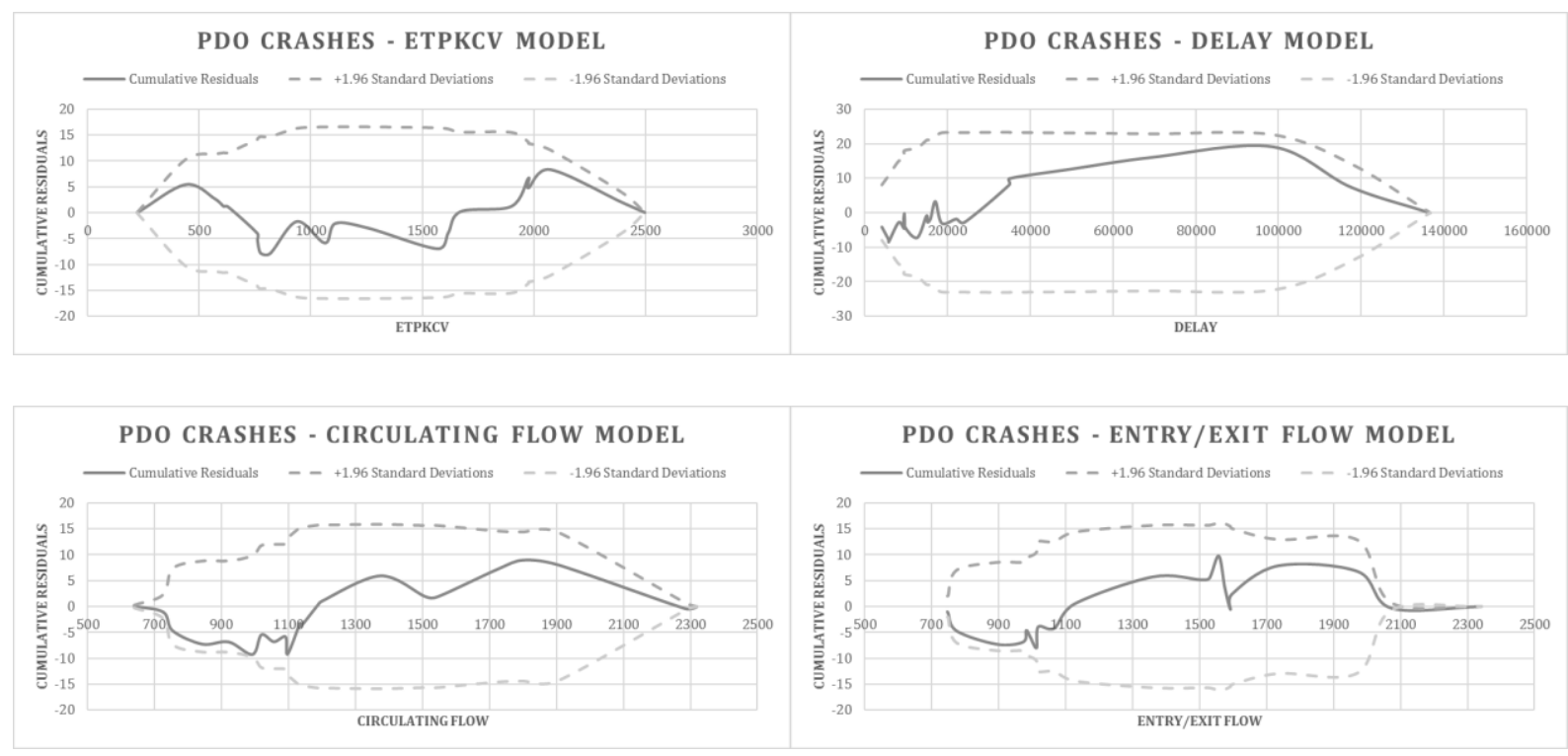

Figure 6.6 CURE Plots for all Model Types (PDO Crashes)

As can be seen from the p-values in Table 6.11, the coefficient estimates are highly significant for all the models. The goodness of prediction measures also show that the MAD/year/site 
(Mean Absolute Deviation) and the MPE/year/site (Mean Prediction Error) for all the models are small when compared to the average observed crashes per year per site. For example, for ETPKCV model the MAD/year/site is 0.56 compared to average observed PDO crashes of $\sim 2$ per year per site. Even though these values are higher than what one would expect them to ideally be, they are comparable to the values obtained by both the circulating flow and the entry/exit flow models. It can also be seen that the ETPKCV model has $\mathrm{K}$ value that is approximately the same as the flow based models showing that the ETPKCV model can capture the over dispersion in the data comparably to the flow based models.

The CURE plots (Figure 6.6) for all models show that the cumulative residuals lie between the 95\% confidence boundaries and oscillate consistently showing little or no bias. The oscillation in these plots is not as one would expect in a perfect CURE plot as described by Hauer (2014), but the main reason for that is, again, the smaller number of sites being used here. Both the goodness of prediction measures and the CURE plots for the models are an indicator of good predictions by all the models.

\subsubsection{Comparative Evaluation of the ETPKCV, Delay \& Flow Based Models}

As can be seen from Sections 6.3.1 and 6.3.2, all models based whether based on ETPKCV, overall Delay, Circulating Flows and/or Entry/Exit Flows predict crashes very well. This fact is affirmed by the highly significant coefficient estimates (as seen by p-values of less than 5\%), the low MAD and MPE values and the CURE plots showing little or no bias in almost all cases. The CURE plots are not as they would be in a perfect scenario as described by Hauer (2014). The main reason behind this is the small number of sites used in this evaluation and to 
an extent it could also be because the small sample used was diverse, consisting of data from three different sources.

For multi-lane models, the coefficient estimates for ETPKCV and delay are slightly smaller than those for the Circulating and Entry/Exit Flows, showing that the models developed are better at capturing the effects of Circulating and Entry/Exit Flows on crashes. On the other hand, even though the ETPKCV and delay coefficient estimates are smaller than those of the Circulating and Entry/Exit Flows, they are still high enough (being close to 1 in most cases) and highly significant. The over dispersion parameter, $\mathrm{K}$, for the ETPKCV models was small compared to the flow based models showing that the ETPKCV models better capture the over dispersion in the data. $\mathrm{K}$ value for delay models were slightly higher that the flow based models but were still comparable. This suggests that the ETPKCV and delay models are able to predict crashes as close to reality. This fact can also be confirmed by comparing the MAD and MPE values in Tables $6.6-6.8$ for the ETPKCV, Total Delay, Circulating Flow, and Entry/Exit Flow models. The MAD/Year/Site and the MPE/Year/Site values are slightly higher than what would be ideal, but are comparable to the values from the flow based models. Even though the CURE plot suggested a slight over prediction by the ETPKCV models in a certain range of conflicting volumes, all the other measures do indicate that the models linking crashes to ETPKCV can predict crashes at multi-lane roundabouts as well as the flow based models.

For single-lane models, the coefficient estimates for both ETPKCV and delay are slightly smaller than those for the Circulating and Entry/Exit Flows, showing that the once again the models developed are better at capturing the effects of Circulating and Entry/Exit Flows on crashes. But it can be seen that the coefficient estimates for the ETPKCV and delay models 
are highly significant. The results also indicate better p-values for injury crashes when ETPKCV and delay models are used as opposed to the flow models. The over dispersion parameter, $\mathrm{K}$, for the ETPKCV models was similar to the flow based models. $\mathrm{K}$ value for the delay models was slightly higher but still comparable to the other models. This suggests that the ETPKCV and delay models are able to predict crashes as close to reality. This fact can also be confirmed by comparing the MAD and MPE values in Tables $6.9-6.11$ for the ETPKCV, Total Delay, Circulating Flow, and Entry/Exit Flow models. The MAD/Year/Site and the MPE/Year/Site values are slightly higher than what would be ideal, but are comparable to the values from the flow based models. The CURE plots in all cases also indicate that the cumulative residuals lie between the $95 \%$ confidence boundaries and that they oscillate consistently, showing little or no bias. The observations indicate that, similar to the multi-lane roundabouts, at single-lane roundabouts the ETPKCV and delay models can again predict crashes as well as the conventional flow based models.

\subsection{Application of GEE Models to Account for Time Trend and/or $\underline{\text { Temporal Correlation }}$}

Both multi- and single-lane roundabout data was used to develop models to account for time trend and/or temporal correlation using General Estimating Equation (GEE). These models will try to capture the year to year change in crashes by taking each year as a separate observation. 


\subsubsection{GEE Models for Multi-Lane Roundabouts}

The model form used for developing the models for multi-lane roundabouts using General Estimating Equations (GEE) was as follows:

Model A: Total Crashes $=e^{\alpha} \times E_{T P K C V}^{\beta_{1}}$

Model B: Total Crashes $=e^{\alpha} \times$ Delay $^{\beta_{1}}$

GEE was used to develop Total Crash models using both ETPKCV (Model A) and Delay (Model B). Both models were developed first without accounting for time trend to compare the outputs with the GLM models developed in Table 6.6. Models were then developed accounting for the time trend and/or temporal correlation. Data used for these models spanned 5 years from 2010 to 2014 . This 5-year time period was used because the data was available for both Region of Waterloo and the Washington State multi-lane roundabouts in this time period.

Table 6.12. Estimates for Multi-Lane GEE Model (with/without Trend)

\begin{tabular}{|c|c|c|c|c|}
\hline Crash Type & \multicolumn{4}{|c|}{ Total (Without Trend) } \\
\hline Model Form & \multicolumn{2}{|c|}{ Model A } & \multicolumn{2}{|c|}{ Model B } \\
\hline Coefficient & Estimate & Pr>ChiSq & Estimate & Pr>ChiSq \\
\hline$\alpha$ & -6.7496 & $<0.0001$ & -3.6656 & $<0.0001$ \\
\hline 81 & 0.9596 & $<0.0001$ & 0.4171 & $<0.0001$ \\
\hline Crash Type & \multicolumn{4}{|c|}{ Total (With Trend) } \\
\hline Model Form & \multicolumn{2}{|c|}{ Model A } & \multicolumn{2}{|c|}{ Model B } \\
\hline Coefficient & Estimate & Pr>ChiSq & Estimate & Pr>Chisq \\
\hline$\alpha($ Year 2010) & -6.8839 & $<0.0001$ & -3.7622 & $<0.0001$ \\
\hline$\alpha($ Year 2011) & -6.8822 & $<0.0001$ & -3.7398 & $<0.0001$ \\
\hline$\alpha($ Year 2012) & -6.6154 & $<0.0001$ & -3.5484 & $<0.0001$ \\
\hline$\alpha($ Year 2013) & -6.6965 & $<0.0001$ & -3.5549 & $<0.0001$ \\
\hline$\alpha$ (Year 2014) & -6.6884 & $<0.0001$ & -3.5574 & $<0.0001$ \\
\hline 81 & 0.9591 & $<0.0001$ & 0.4136 & $<0.0001$ \\
\hline
\end{tabular}


As can be seen from Table 6.12, the GEE models (not accommodating for trend) portray similar results as the GLM models for Total Crashes shown in Table 6.6. The slight differences in the estimates can be attributed to the fact the GLM models were developed using cumulative crash data, whereas, the GEE models were developed using yearly crash data. Thus even though we are not accounting for trend, the number of data points used in GEE models would be five times as much as used in the GLM models (based on 5 years of crash data used). The GEE models (accommodating for trend) estimate separate coefficients for each year of crash data used. It can be seen that the trend in the intercept estimates closely resembles to the trend in Total Crashes (observed by year) as shown in Table 6.5.

\subsubsection{GEE Models for Single-Lane Roundabouts}

The model form used for developing the models for single-lane roundabouts using General Estimating Equations (GEE) was as follows:

Model A: Total Crashes $=e^{\alpha} \times E T P K C V^{\beta_{1}}$

Model B: Total Crashes $=e^{\alpha} \times$ Delay $^{\beta_{1}}$

GEE was used to develop Total Crash models using both ETPKCV (Model A) and Delay (Model B). Both models were developed first without accounting for time trend to compare the outputs with the GLM models developed in Table 6.9. Models were then developed accounting for the time trend and/or temporal correlation. Data used for these models spanned 6 years from 2008 to 2013. This 6-year time period was used because the data was available for both City of Ottawa and the Washington State single-lane roundabouts in this time period. 
As can be seen from Table 6.13, the GEE models (not accommodating for trend) portray similar results as the GLM models for Total Crashes shown in Table 6.9. The slight differences in the estimates can be attributed to the fact the GLM models were developed using cumulative crash data, whereas, the GEE models were developed using yearly crash data. Thus even though we are not accounting for trend, the number of data points used in GEE models would be six times as much as used in the GLM models (based on 6 years of crash data used).

Table 6.13 Estimates for Single-Lane GEE Model (with/without Trend)

\begin{tabular}{|c|c|c|c|c|}
\hline Crash Type & \multicolumn{4}{|c|}{ Total (Without Trend) } \\
\hline Model Form & \multicolumn{2}{|c|}{ Model A } & \multicolumn{2}{|c|}{ Model B } \\
\hline Coefficient & Estimate & Pr>Chisq & Estimate & Pr>ChiSq \\
\hline$\alpha$ & -4.8830 & $<0.0001$ & -3.4178 & $<0.0001$ \\
\hline 81 & 0.5118 & $<0.0001$ & 0.2202 & $<0.0001$ \\
\hline Crash Type & \multicolumn{4}{|c|}{ Total (With Trend) } \\
\hline Model Form & \multicolumn{2}{|c|}{ Model A } & \multicolumn{2}{|c|}{ Model B } \\
\hline Coefficient & Estimate & Pr>Chisq & Estimate & Pr>Chisq \\
\hline$\alpha($ Year 2008) & -5.4169 & $<0.0001$ & -4.0526 & $<0.0001$ \\
\hline$\alpha$ (Year 2009) & -4.9984 & $<0.0001$ & -3.5857 & $<0.0001$ \\
\hline$\alpha($ Year 2010) & -4.5831 & $<0.0001$ & -3.1848 & $<0.0001$ \\
\hline$\alpha($ Year 2011) & -4.8623 & $<0.0001$ & -3.4469 & $<0.0001$ \\
\hline$\alpha($ Year 2012) & -5.0527 & $<0.0001$ & -3.6128 & $<0.0001$ \\
\hline$\alpha($ Year 2013) & -4.7355 & $<0.0001$ & -3.3454 & $<0.0001$ \\
\hline 81 & 0.5161 & $<0.0001$ & 0.2236 & $<0.0001$ \\
\hline
\end{tabular}

The GEE models (accommodating for trend) estimate separate coefficients for each year of crash data used. It can be seen that the trend in the intercept estimates closely resembles to the trend in Total Crashes (observed by year) as shown in Table 6.5. Furthermore, it can be seen that due to the GEE model accounting for the time trend, the coefficient estimate for TPKCV is slightly higher compared to the GLM models predicting that the model is capturing the effects of the conflicting volumes slightly better when time trend in accommodated. 


\subsection{Chapter Summary}

The main purpose of this chapter was to answer the question of whether it is worthwhile to collect turning movement counts at roundabouts. To this end, the research evaluated roundabout crash models based on the estimated total peak hour conflicting volume (ETPKCV) and the total overall roundabout delay and compared them with the more traditional flow based models. All of the models developed showed that the coefficient estimates for the variables were statistically significant in almost all cases at the $5 \%$ level. The MAD/year/site and the MPE/year/site values even though were slightly higher, but were comparable to the flow based models. The dispersion parameters for both the TDCV and flow based models are small and similar to each other. The CURE plots also suggest of good fit since the residuals stay well within the $95 \%$ confidence boundaries.

The ETPKCV and delay based models, however, yield significant coefficient estimates for the ETPKCV and total delay but the effect of both these variables is weak as can be seen by lower coefficient estimates when compared to the estimates of the flow based models. The weak effect of these variables on the crashes can be explained partially by the different regions and the number of sites used in the study. A higher number of sites and using data from only one region might have yielded different results.

The GEE models (both multi- and single-lane) without trend showed similar results to the GLM models with slight differences being attributable to the way the data is used for both models (i.e. cumulative crash data for GLM and each year separately for GEE). Both multiand single-lane models accommodating for trend resembles the observed crash data (recorded 
by year) and slightly better coefficient estimates due to the time trend and/or temporal correlation being accounting for.

To conclude, it can be said that ETPKCV and delay based crash prediction models provide a good alternative to the flow based models and that they can be effectively used to evaluate the safety of multi- and single-lane roundabouts. Based on this conclusion, it seems worthwhile to collect turning movement counts at roundabouts, especially considering that they are required for Highway Capacity Manual capacity and level of service calculations. Future research can further focus on the potential of using conflicting volumes and delay to better estimate safety at roundabouts and better methods can be researched to estimate the conflicting volumes and delay and subsequently improving the models developed in this dissertation. 


\section{Chapter 7. Accomplishments, Conclusions and Future Work}

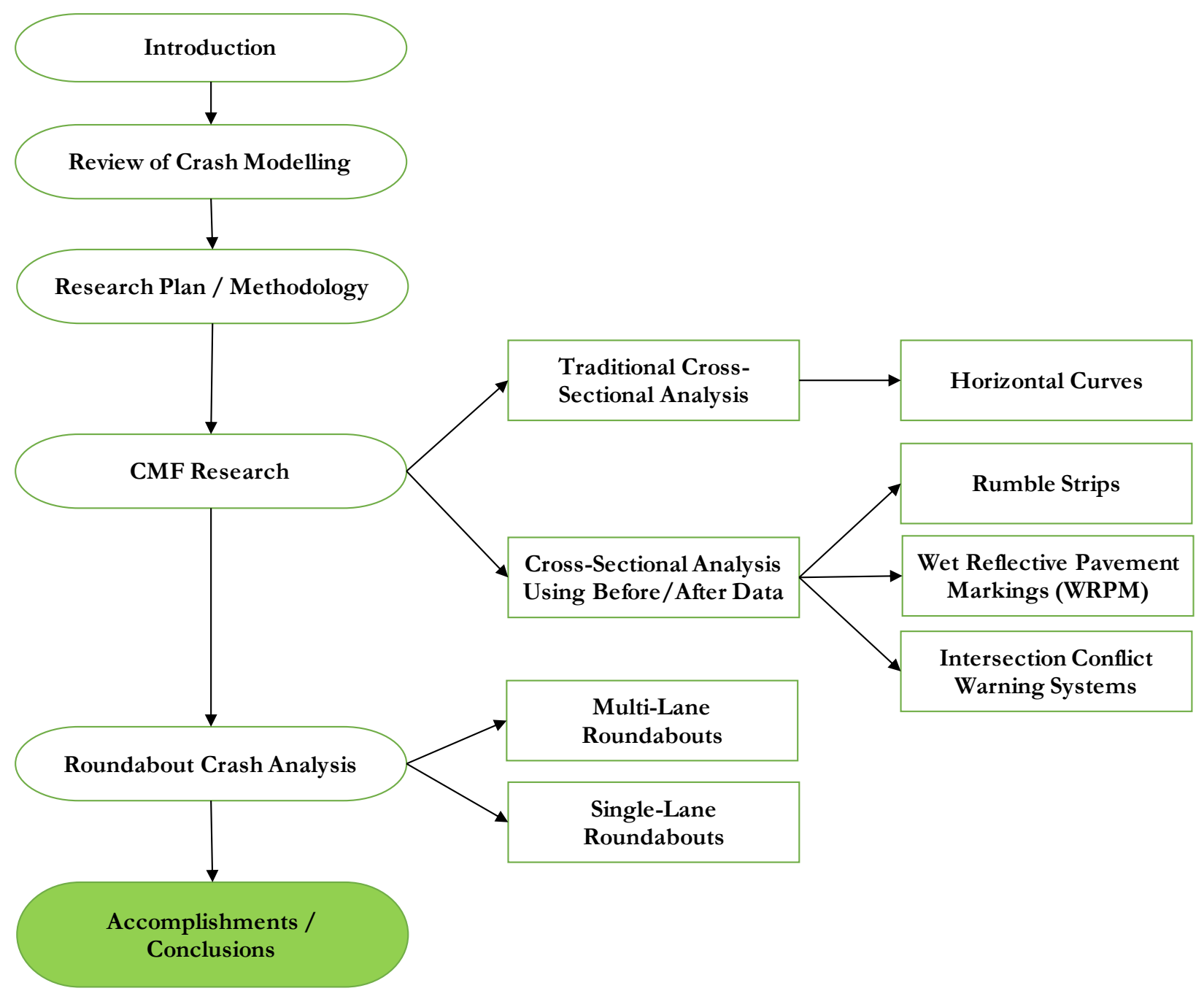

Chapter 4-6 have contributed in filling the gaps in the current research by evaluating the potential of cross-sectional analysis to better estimate the safety effects both in the absence and presence of before-after data and by introducing new approaches to evaluate the relationship between safety and traffic operations at roundabouts incorporating the use of rarely collected turning movement counts. This chapter is the conclusive summary of contents 
in Chapters 4-6 and consists of three sections: summary and conclusions, accomplishments and recommendation for future studies.

\subsection{Summary and Conclusions}

This research was divided into two components. The first component looked at evaluating the potential of cross-sectional analysis for developing crash modification factors/functions (CMFs/CMFunctions) used to represent the effects of safety treatments on crashes. The object here was to evaluate the potential of cross-sectional analysis in the absence of before-after data, to see whether cross-sectional analysis can predict results comparably to before-after analysis (for cases where before-after data is available), and, finally, to see if cross-sectional analysis can better predict CMFunctions even in the presence of before-after data. The second component looked at the development of roundabout crash prediction models using conflicting volumes and delays, which could only be estimated from turning movement counts that are rarely collected at roundabouts. The object here was to determine whether the considerable extra effort to collect these data would be worthwhile. In doing so, this research also addresses advancing both the safety performance functions (SPFs) and CMFs.

The first part of the CMF research in this dissertation looked at evaluating the potential of cross-sectional analysis in the absence of before-after data. The design element for this part was horizontal curvature on rural two-lane highways for which collecting before-after data would be impossible because of the rarity of this treatment and the infinite combinations of curvature before and after. This part of the research provided new insights into estimating CMFs for flattening an existing horizontal curve on rural 2-lane highways. Crash prediction 
models were developed for the curve sections and also the tangents up and down stream of the curve sections on level $(<3 \%)$, moderate $(3 \%-6 \%)$, and steep $(>6 \%)$ grades. The model parameters were statistically significant to the 5\% level and had low standard errors. The goodness of prediction measures also suggested a good fit. To further demonstrate the capabilities of this approach, the relationship between crashes at different volumes and deflection angles were also explored to get approximate estimates of CMFs for increasing the minimum radius required by a factor of $1.10,1.25,1.50$, and 2.00 . In this case, to accurately compare crashes between the old curve and the flattened curve, crashes on the tangent section that was removed to accommodate the longer length of the flattened curve were also taken into account. The results show that even at different design speeds, the CMF estimates for a certain scenario lie in the same range conforming to the findings from Zegeer et al. (1992) and Hauer (1999). The results also showed that the effect of changing horizontal curvature was captured as being much larger when the data was explored without confounding effects of gradient. Overall, these results show that cross-sectional analysis can be used effectively to capture the effect of a treatment in cases where before-after are either not available or are impossible to obtain.

The second part of the CMF research in this dissertation looked into further evaluating the potential of the cross-sectional approach by estimating CMFs from cross-sectional studies using actual before-after databases and compare these estimates to those from empirical Bayes before-after studies. This part also looked at the potential of cross-sectional analysis to better estimate CMFunctions than before-after studies. The design elements for this investigation were combination (center line plus shoulder) rumble strips, wet reflective pavement markings 
in various road class categories and intersection conflict warning systems. The data used came from Minnesota, North Carolina, Kentucky, Missouri, Pennsylvania, and Wisconsin Departments of Transportation. The data included both treatment sites (where wet reflective pavement markings, combination rumble strips and intersection conflict warning systems were installed, with the installation dates) and reference sites (similar to treated sites in characteristics but without the treatment). A cross-sectional analysis was conducted using negative binomial regression and a combination of the reference site data and the after period data at the treatment sites to derive functions that can be subsequently be used to estimate CMFs. These CMFs were then compared to recent studies by Persaud et al. (2016), Lyon et al. (2016), and Himes et al. (2016), where the three treatments used were evaluated using empirical Bayes before-after analysis with the same datasets used for the cross-sectional regression.

Based on the three case studies done for the second part of the CMF research in this dissertation, it can be seen that the cross sectional models that were developed, using a combination of the reference site data and the after period data at the treatment sites, can not only estimate CMFs that are close and comparable to the CMFs estimated from empirical Bayes before-after studies but also have the potential to better capture the variation of the CMF with certain factors. This also opens the door for further research in this area whereby more treatments can be explored to see whether similar results can be achieved. This part of the research answered the three main questions that formed the motivation for undertaking it, i.e., that carefully done cross-sectional regression can infer the effects of treatments, that they can 
provide CMF estimates close to the CMF estimates from before-after analysis, and that they can better estimate CMFunctions even in the presence of before-after data.

The second component of this research involved a comparative analysis of roundabout crash predictions using estimated total peak hour conflicting volume (ETPKCV) and delays (both of which can only be calculated using rarely collected turning movement counts at roundabouts) and the traditional flow based models. Crash prediction models were developed based on (a) estimated total peak hour conflicting volumes (ETPKCV), (b) circulating flow, (c) entry/exit flow, and (d) total overall delay.

Based on the comparative analysis of these models, it can be said that ETPKCV and delay based crash prediction models provide a good alternative to the flow based models and that they can be effectively used to evaluate the safety of multi- and single-lane roundabouts. Based on this conclusion, it seems worthwhile to collect turning movement data at roundabouts and opens the door to future research to further focus on the potential of this approach.

\section{$\underline{7.2 \text { Accomplishments }}$}

Safety evaluations using cross-sectional analysis and before-after evaluations are not a new topic in traffic safety domain. The basic framework for conducting these analysis has been well established and widely applied in practices in the real world for a long amount of time. Nevertheless, there are still many advocates who strongly believe that cross-sectional analysis can not predict the safety of an entity as well as before-after analysis.

Past research may have concentrated heavily on the development of safety prediction models while paying insufficient attention to dealing with the issue of consistency between cross- 
sectional and before-after analysis. This dissertation has sought to balance this component of research by validating the traditional cross-sectional analysis and evaluating the consistency between cross-sectional and before-after analysis.

In order to achieve this balance, this dissertation first looks at evaluating the potential of crosssectional analysis to estimate CMFs in the absence of before-after data. In doing so, new insights were provided on estimating safety effects of horizontal curvature on rural two-lane highways. The results from this evaluation were validated against some prominent research work on horizontal curvature and the comparison was found to be favourable.

This dissertation then moves on towards evaluating the potential of cross-sectional analysis to better estimate CMFs using actual before-after study databases in order to see whether crosssectional analysis can predict results comparably to before-after analysis. Three case studies involving combination (center line plus shoulder) rumble strips, wet reflective pavement markings and intersection conflict warning systems were conducted to bridge this gap. This evaluation made use of a combination of untreated reference sites and after period data from treatment sites to conduct cross-sectional analysis and compared the results to recent empirical Bayes studies conducted using datasets that were used in this dissertation. The comparison shows similar trends in the safety estimates from both cross-sectional and before-after analysis. The results from this evaluation suggest that cross-sectional analysis can be used to evaluate safety comparably to before-after analysis.

Furthermore, throughout the analysis, cross-sectional models were developed not only to achieve a single value safety effect (CMFs), but also to explore interactions between various 
roadway components and the treatment in question. This lead to converting the single value CMFs into CMFunctions, whereby, significant interactions of various components play an important role in getting better/more accurate estimate of the effects on safety both locally for the dataset in question and universally where these CMFunctions are applied. The results show that cross-sectional analysis have the potential to better capture the variation of the CMF with certain factors even in the presence of before-after data.

This dissertation then moves towards doing a comparative analysis of roundabout crash predictions using conflicting volumes and delays (both of which can only be calculated using rarely collected turning movement counts) and the traditional approach flow based models. The purpose here was to assess whether it is worth the extra effort to collect turning movement data that are not typically collected at roundabouts. The evaluation found that the new approaches can predict safety comparably to the traditional flow based models and opens the door for further research in this area. The results show that it is worthwhile to put forth the extra effort needed to collect turning movement counts at roundabouts.

Overall, this dissertation tried to balance/fill the gaps in current research in a bid to achieve better overall approaches for estimating safety impacts of highway design and operational elements by demonstrating the use simpler statistical techniques to reach comparable conclusions to those from the rather sophisticated techniques that can be used. This allows practitioners to better undertake such research by using simpler analytical tools, thus widening the possible use of analytics in the applied world of traffic safety. 


\section{$\underline{7.3 \text { Future Work }}$}

This dissertation presents an analysis on how to integrate different aspects of research to achieve better approaches to estimate safety impacts. However, this research can be enhanced further and extended in many ways. Traditional cross-sectional analysis to evaluate safety at horizontal curves only made use of HSIS data for Washington State. The reason behind this was that amongst the databases available, the Washington database provided the most detailed information on horizontal curves. As more data from different states become available in the future, work can be done on calibrating the models developed in this dissertation to those other datasets, in assessing transferability of the results.

Cross-sectional analysis to estimate CMFs using actual before-after study databases was only done for three treatments. Promising results showing consistency between estimates from cross-sectional and before-after analysis opens the door for further research in this area whereby more treatments can be explored using the approach used in this research to see whether similar results can be achieved.

Safety surrogates, such as traffic conflicts, can be used to simulate the after treatment scenario for treatments where it is impossible to obtain before-after data. Future work can look at developing a mechanism to integrate the use of simulation to complement the traditional crosssectional analysis in obtaining a more precise safety effect. Finally, new approaches to estimating safety at roundabouts presented in this dissertation can be enhanced further in the future by incorporating more dynamic safety attributes in traffic operations and by better addressing local specifics with inclusion of more flexible parameters into the models. 


\section{$\underline{\text { Reference List }}$}

AASTHO (2010). Highway Safety Manual, $1^{\text {st }}$ Edition, AASTHO, Washington, DC.

Aguero-Valverde, J. and Jovanis, P.P. (2009). Bayesian Multivariate Poisson LogNormal Models for Crash Severity Modeling and Site Ranking. Transportation Research Record. 2136, pp. 82-91, 2009.

Bahar, G., Masliah, M., Erwin, T., Tan, E., and Hauer, E. (2006). Pavement Marking Materials and Markers: Real World Relationship between Retro Reflectivity and Safety over Time. NCHRP Web Only Document 92, 2006.

Bahar, G., Parkhill, M., Tan, E, Philp C., Morris, N., Naylor, S., and White, T. (2007) NCHRP 17-27: Inclusion Process and Literature Review for Part D. iTrans Consulting, 2007.

Bauer, K., and Harwood, D., (2013). Safety Effects of Horizontal Curve and Grade Combinations on Rural Two-Lane Highways. Report No. FHWA-HRT-13-077, Federal Highway administration, Washington, DC.

Bonneson, J.A., and Pratt, M.P. (2008). Procedure for Developing Accident Modification Factors from Cross-Sectional Data. Transportation Research Record: Journal of the Transportation Research Board, No. 2083, pp. 40-48, 2008.

Bretherton, W. and Miao, J. (1999). Proposed Guidelines for Traffic Warning Signs at Intersections with Limited Sight Distance. Available Online at: http://enterprise.prog.org/Projects/2010_Present/developingconsistencyIWS/GAGwinnettCo untyProject1999.pdf

Cameron, A.C. and Trivedi, P.K. (1998). Regression Analysis of Count Data. Econometric Society Monographs, Vol. 30, New York: Cambridge University Press, 1998. 
Carlson, P., Park E., and Kang, D. (2015). Nighttime Safety and Pavement Marking Retro Reflectivity on Two-Lane Highways: Revisited with North Carolina Data. TRB 94th Annual Meeting Compendium of Papers CD-ROM. Washington, DC.

Carter, D., Srinavasan, R., Gross, F., and Council, F. (2012), Recommending Protocols for Developing Crash Modification Factors. NCHRP 20-7(314) Final Report, 2012.

Chen, Y. (2013). Integrating Information from Prior Research into a Before-after Road Safety Evaluation. PhD Thesis, Ryerson University, 2013.

Chin, H.C. and Huang, H. (2008). Modeling Multilevel Data in Traffic Safety: A Bayesian Hierarchical Approach. Transportation Accident Analysis and Prevention, Nova Science Publishers, Inc., New York, pp.53-106, 2008.

Davis, G.A. (2004). Possible Aggregation Biases in Road Safety Research and a Mechanism Approach to Accident Modeling. Accident Analysis and Prevention, Vol. 36, pp. 1119-1127, 2004.

Diggle, P.J., Liang, K.Y., and Zeger, S.L. (1994). Analysis of Longitudinal Data. Clarendon Press, Oxford, U.K., 1994.

Donnell, E.T., Karwa, V., and Sathyanarayanan, S. (2009). A Methodology to Explore the Relationship between Pavement Marking Retro Reflectivity and Traffic Crash Frequency on Highways in North Carolina: Application of Artificial Neural Networks and the Generalized Estimating Equations. TRB 88th Annual Meeting Compendium of Papers CDROM. Washington, DC.

Donnnell, E.T., Karwa, V., and Slavkovic, A.B. (2011). Causal Inference in Transportation Safety Studies: Comparison of Potential Outcomes and Causal Diagrams. Annals of Applied Statistics, Vol. 5, No. 2B, pp. 1428-1455, 2011.

Elbasyouny, K (2011). New Techniques for Developing Safety Performance Functions. $\mathrm{PhD}$ Thesis, University of British Columbia, 2011. 
Elvik, R. (2009). Developing Accident Modification Functions. Exploratory Study. Transportation Research Record, 2103, pp. 18-24, 2009.

Elvik, R. (2011a). Crash Modification Factors versus Functions, Presented at the International Workshop on Transferability of Crash Modification Factors, TRB 2011 Annual Meeting, January 23rd, 2011.

Elvik, R. (2011b). Assessing Causality in Multivariate Accident Models. Accident Analysis and Prevention, Vol. 43, pp. 253-264, 2011.

Elvik, R. (2013). International Transferability of Accident Modification Functions for Horizontal Curves. Accident Analysis and Prevention, Vol.59, pp. 487-496, 2013.

Fitzpatrick, K., Lord, D., and Park, B.J., (2009). Horizontal Curve Accident Modification Factor with Consideration of Driveway Density on Rural, Four-Lane Highways in Texas. TRB 88th Annual Meeting Compendium of Papers CD-ROM. Washington, DC.

Gross, F., Persaud, B., and Lyon, C. (2010). A Guide to Developing Quality Crash Modification Factors, FHWA-SA-10-032, U.S. Department of Transportation, Federal Highway Administration, Washington, DC, December 2010.

Gross, F., and Donnell, E.T. (2011). Case-Control and Cross-Sectional Methods for Estimating Crash Modification Factors: Comparison from Roadway Lighting and Lane and Shoulder Width Safety Effect Studies. Journal of Safety Research, Vol. 42 (2), pp. 117-129, 2011.

Hanscom, F. (2001). Evaluation of the Prince William County Collision Countermeasure System. Report No. FHWA/VTRC 01-CR5, Virginia Transportation Research Council, VA, 2001.

Hauer, E., and Persaud, B. (1983). Common Bias in Before-and After Accident Comparison and Its Elimination. Transport Research Record: Journal of the Transportation Research Board, No. 905, pp. 164-174, 1983. 
Hauer, E. (1994). The Effect of Resurfacing on the Safety of Two-Lane Roads in New York State. Report to the New York State Transportation Department, University of Toronto, 1993.

Hauer, E. (1997). Observational Before-After Studies in Road Safety. Elsevier Science, Oxford, UK, 1997.

Hauer, E. and Bamfo, J. (1997). Two Tools for Finding What Function links the Dependent Variable to the Explanatory Variables. Proceedings of ICTCT 1997 Conference, Lund.

Hauer, E., (1999). Safety and the Choice of Degree of Curve. Transport Research Record: Journal of the Transportation Research Board, No. 1665, pp. 22, 1999.

Hauer, E., Harwood, D.W., Council, F.M., Hughes, W.E., and Vogt, A., (2000). Prediction of the Expected Safety Performance of Rural Two-Lane Highways, Report No. FHWA-RD-99-207, Federal Highway Administration, Washington, DC.

Hauer, E., Bonneson, J.A., Council, F., Srinavasan, R., Zeeger, C. (2012). Crash Modification Factors: Foundational Issues. Transportation Research Record: Journal of the Transportation Research Board, No. 2279, pp. 67-74, 2012.

Hauer, E. (2014). The Art of Regression Modelling in Road Safety. Springer International Publishing, Switzerland, 2015.

Himes, S., Gross, F., Eccles, K., and Persaud, B. (2016). Multi-State Safety Evaluation of Intersection Conflict Warning Systems (ICWS). TRB 95th Annual Meeting Compendium of Papers CD-ROM. Washington, DC.

HSIS (2016). Highway Safety Information System. Available Online at: http://www.hsisinfo.org/ 
Isebrands, H. (2011). Quantifying Safety and Speed for Rural Roundabouts with HighSpeed Approaches. PhD Dissertation. Iowa State University.

Kay, J., Savolainen, P. T., Gates, T. J., Datta, T. K., Finkelman, J., and Hamadeh, B. (2015). Safety Impacts of a Statewide Centerline Rumble Strip Installation Program. Transportation Research Record: Journal of the Transportation Research Board, No. 2515, pp. 34-40, 2015.

Lee, J., Chung, J., and Son, B. (2008). Analysis of Traffic Accident Size for Korean Highway using Structural Equation Models. Accident Analysis and Prevention, Vol. 40, pp. 1955-1963, 2008.

Lord, D., Washington, S.P. and Ivan, J.N. (2005). Poisson, Poisson-Gamma and ZeroInflated Regression Models of Motor Vehicle Crashes: Balancing Statistical Fit and Theory. Accident Analysis and Prevention, Vol. 37, pp. 35-46, 2005.

Lord, D (2006). Modeling Motor Vehicle Crashes using Poisson-Gamma Models: Examining the Effects of Low Sample Mean Values and Small Sample Size on the Estimation of the Fixed Dispersion Parameter. Accident Analysis and Prevention, Vol. 38, Issue 4, pp. 751-766, 2006.

Lord, D. and Miranda-Moreno, L.F. (2008a). Effects of Low Sample Mean Values and Small Sample Sizes on the Parameter Estimation of Hierarchical Poisson Models for Motor Vehicle Crashes: A Bayesian Perspective. Safety Science, Vol. 46, pp. 751-770, 2008.

Lord, D., Persaud, B., Washington, S. P., Ivan, J. N., Lyon, C. and Jonson, T. (2008b). Methodology to Predict the Safety Performance of Rural Multilane Highways. NCHRP Online Report 126. Transportation Research Board, Washington, DC, pp. 53 - 56. Available online at: http://onlinepubs.trb.org/onlinepubs/nchrp/nchrp_w126.pdf. 
Lord, D. and Persaud, B. (2000) Accident Prediction Models With and Without Trend. Transport Research Record: Journal of the Transportation Research Board, No. 1717, pp. 102$108,2000$.

Lord, D., and Kuo, P.-F. (2012). Examining the Effects of Site Selection Criteria for Evaluating the Effectiveness of Traffic Safety Countermeasures. Accident Analysis \& Prevention, Vol. 47, pp. 52-63, 2012.

Lyon, C., Persaud, B., Haq, A., and Kodama, S. (2006). Safety Performance Functions for Signalized Intersections in Large Urban Areas: Development and Application to Evaluation of Left Turn Priority Treatment. Transport Research Record: Journal of the Transportation Research Board, No. 1908, pp. 165-171, 2006.

Lyon, C., Persaud, B., and Eccles, K. (2016). Safety Evaluation of Wet Reflective Pavement Markings. TRB 95th Annual Meeting Compendium of Papers CD-ROM. Washington, DC.

Matthew, G and Golias, I (2002). Effects of Road Geometry and Traffic Volumes on Rural Roadway Accident Rates. Accident Analysis and Prevention, Vol, 34, Issue 3, pp. 35765, 2002.

Milton, J.C., Shankar, V., and Mannering, F., (2008). Highway Accident Severities and the Mixed Logit Model: An Exploratory Empirical Analysis. Accident Analysis and Prevention, Vol.40, Issue 1, pp. 260-266, 2008.

Miranda-Moreno, L.F. (2006). Statistical Models and Methods for Identifying Hazardous Locations for Safety Improvements. Ph.D. Dissertation, University of Waterloo, 2006.

Missouri Department of Transportation (2010). Missouri DOT Intersection Warning System Before-After Study Results. 2012. 
Mountain, L., Maher, M.J., and Fawaz, B. (1998). The Influence of Trend on Estimates of Accidents at Junctions. Accident Analysis and Prevention, Volume 30 (5), pp. 641-649, 1998.

OECD (2012). Sharing Road Safety: Developing an International Framework for Crash Modification Functions. Report No. ITF/OECD/JTRC(2012)7, March 2012.

Park, P.Y., Miranda-Moreno, L., and Saccomanno, F.F. (2010). Estimation of Speed Differentials on Rural Highways using Hierarchical Linear Regression Models. Canadian Journal of Civil Engineering, Volume 37(4), pp. 624-637, 2010.

Patterson, B (2009). Ontario Roads Safest in North America. Government of Ontario Newsroom. April 29. 2009. Available Online at: http://news.ontario.ca/mto/en/2009/04/ontarios-roads-safest-in-north-america.html

Pennsylvania Department of Transportation (2007). Collision Avoidance System Evaluation Report, 2007.

Persaud, B., Saleem, T., Lyon, C., and Chen, Y. (2012). Safety Performance Functions for Estimating the Safety Benefits of Proposed or Implemented Countermeasures. A report prepared for Transport Canada under Canada's National Road Safety Research and Outreach Program, Ryerson University, 2012.

Persaud, B and Dzbik, L. (1993). Accident Prediction Models for Freeways. Transport Research Record: Journal of the Transportation Research Board, No. 1401, pp. 50-60, 1993.

Persaud, B., Lyon, C., Eccles, K., and Soika, J. (2016). Safety Effectiveness of Center Line Plus Shoulder Rumble Strips on Two-Lane Rural Roads. Journal of Transportation Engineering, 4016012. doi:10.1061/(ASCE)TE.1943-5436.0000821.

Pellecuer, L. and St-Jacques, M. (2008). Latest Advances on Roundabouts. Rev. Can. Genie Civ., Vol. 35, page. 542-553. 
Pitale, J.T., Shankwitz, C., Preston, H., and Barry, M., (2009). Benefit-Cost Analysis of In-Vehicle Technologies and Infrastructure Modifications as a Means to Prevent Crashes along Curves and Shoulders. Minnesota Department of Transportation.

Rodegerdts, L., Blogg, M., Wemple, W., Myers, E., Kyle, M., Dixon, M., et al. (2007). Roundabouts in the United States. Report 572. Transportation Research Board - National Cooperative Highway Research Program, Washington DC, USA.

SAS (2016). SAS: Enterprise Guide. Available Online at: http://www.sas.com/technologies/bi/query_reporting/guide/

Saleem, T. (2012). Evaluation of the Predictive Capabilities of Simulated Peak Hour Conflict Based Crash Prediction Models. MASc Thesis, Ryerson University, 2012.

Sayed, T., deLeur, P., and Pump, J. (2010). Impact of Rumble Strips on Collision Reduction on Highways in British Columbia, Canada. Transportation Research Record: Journal of the Transportation Research Board, No. 2148, pp 9-15. 2010.

Schneider, W.H., Zimmerman, K., Boxel, D.V., and Vavilikolanu, S., (2009). A Bayesian Analysis of the Effect of Horizontal Curvature on Truck Crashes Using Training and Validation Data Sets. TRB 88th Annual Meeting Compendium of Papers CD-ROM. Washington, DC.

Shankar, V., Mannering, F., and Barfield, W. (1996). Statistical Analysis of Accident Severity On Rural Freeways. Accident Analysis and Prevention, Vol. 3, pp. 391-401, 1996.

Smadi, O., Souleyrette, R.R., Ormand, D.J., and Hawkins, N. (2008). An Analysis of the Safety Effectiveness of Pavement Marking Retro Reflectivity. TRB 87th Annual Meeting Compendium of Papers CD-ROM. Washington, DC.

Smith, T.C., Spiegelhalter, D.J., Thomas, A. (1995). Bayesian Approaches to Random Effects Meta-Analyses: A Comparative Study. Statistics in Medicine, Vol. 14, pp. 2685-2699, 1995. 
TAC (1999). Geometric Design Guide for Canadian Roads. Transportation Association of Canada, Ottawa.

Thavaneswaran, A., and Lix, L. (2008). Propensity Score Matching in Observational Studies. Report Prepared for Manitoba Centre for Health Policy, University of Manitoba, 2008.

Torbic, D., Bauer, K., Hutton, J., and Campbell, J. (2013). Delta Region Transportation Development Program: Rural Safety Innovation Program Evaluation: Final Report, US Dept. of Transportation, Federal Highway Administration, Washington, DC.

Transport Canada (2006). Safe, Safer, Safest: Road Safety Vision 2010. TP 14611 (09/2006), TC-1002060, ISBN 0-662-49492-X. Available Online at: http://www.tc.gc.ca/eng/roadsafety/tp-tp14611-menu-836.htm

Transport Canada (2007). Analysis and Estimation of the Social Cost of Motor Vehicle Collisions in Ontario. TP 14800E (08/2007). Available Online at: http://www.tc.gc.ca/eng/roadsafety/tp-tp14800-menu-159.htm

Transport Canada (2013). Canadian Motor Vehicle Collision Statistics: 2013. Available online at: https://www.tc.gc.ca/media/documents/roadsafety/cmvtcs2013_eng.pdf

TRB (2010). Highway Capacity Manual 2010 Version. Transportation Research Board, Washington D.C., 2010.

TRB (2010a). Roundabouts: An Informational Guide. NCHRP Report 672. Transportation Research Board, Washington D.C., 2010.

TRB (2016). Development of Roundabout Crash Prediction Models and Methods. NCHRP Project 17-70 (Active). Transportation Research Board, Washington D.C., 2016.

Washington, S., Leonard, L., Manning D.G., Roberts, C., Williams, B., Bacchus, A.R., Devanhalli, A., Ogle, J., and Melcher, D. (2001), Scientific Approaches to Transportation Research Volumes 1 and 2. NCHRP Online Report 20-45, Transportation Research Board, 
National Cooperative Highway Research Program, Washington, DC. Available online at: http://onlinepubs.trb.org/Onlinepubs/nchrp/cd-22/start.htm

Winkelmann, R. (2003). Econometric Analysis of Count Data. Springer, Germany, 2003.

World Health Organization (2013). Global Status Report on Road Safety 2013, ISBN 92-4-156384-0, World Health Organization, Geneva, 2013.

Wu, L., Lord, D., and Zou, L. (2015). Validation of CMFs Derived from CrossSectional Studies using Regression Models. TRB 94th Annual Meeting Compendium of Papers CD-ROM. Washington, DC.

Zegeer, C., Stewart, J., Council, F., Reinfurt, D., and Hamilton, E., (1992). Safety Effects of Geometric Improvements on Horizontal Curve. Transport Research Record: Journal of the Transportation Research Board, No. 1356, pp. 11-19, 1992. 